Reaeration in

Open-Channel Flow

GEOLOGICAL SURVEY PROFESSIONAL PAPER 737

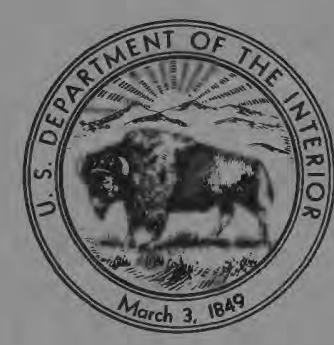




\section{Reaeration in}

Open-Channel Flow

By J. P. BENNETT and R. E. RATHBUN

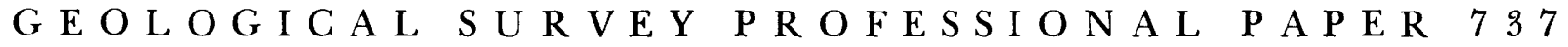

An evaluation of procedures for

measuring and predicting the

reaeration coefficient of

open-channel flows

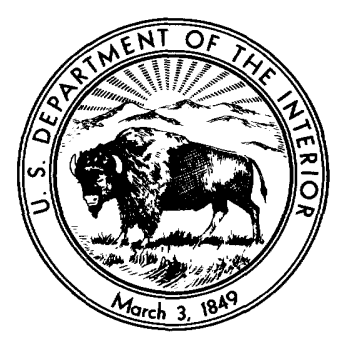




\section{UNITED STATES DEPARTMENT OF THE INTERIOR \\ ROGERS C. B. MORTON, Secretary}

GEOLOGICAL SURVEY

V. E. McKelvey, Director

Library of Congress catalog-card No. 72-600020

For sale by the Superintendent of Documents, U.S. Government Printing Office Washington, D.C. 20402 - Price $\$ 1$ (Paper Cover)

Stock Number 2401-2146 


\section{CONTENTS}

Abstract

Introduction

Theories for predicting the reaeration coefficient.

Diffusion equation.

Conceptual models for the gas-liquid mass-transfer process.

Film model.

Renewal models

Penetration model.

Film-penetration model

Surface renewal-damped-eddy

diffusivity model

Kinetic theory.

Large-eddy model..

Turbulent-diffusion model

Semiempirical models.

Thackston model

Empirical prediction equations

Streeter-Phelps equations.

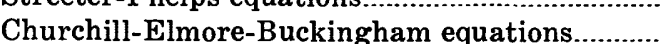

Owens-Edwards-Gibbs equation.

Langbein-Durum equation.

Isaacs-Gaudy equations..

Negulescu-Rojanski equations.

Experimental determination of the reaeration coefficient.

Measurement of dissolved oxygen in water

Techniques for determining the reaeration coefficient

Dissolved-oxygen balance technique

BOD test and $K_{1}$.

Bottom deposits, $K_{3}$, and $m$.

Photosynthesis and respiration.

Other parameters.

Calculation of $k_{2}$ in a recirculating flume.
Page

1

7

10

10

11

13

14

15

16

17

18

18

19

19

20

20

21

21
Experimental determination of the reaeration coefficient - Continued

Techniques for determining the reaeration coefficient - Continued

Disturbed-equilibrium technique..

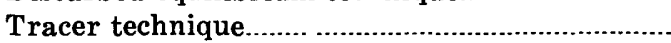

Analysis of errors in reaeration-coefficient calculation techniques..

Accuracy of measured dissolved-oxygen balance parameters.

Dissolved-oxygen balance technique.

Disturbed-equilibrium technique

Tracer technique.

Laboratory measurements....

Prediction of the reaeration coefficient.

Reaeration-coefficient data sets.

Field data

Flume data

Evaluation of reaeration-coefficient prediction equations

Development of a reaeration-coefficient prediction equation

Regression analysis.

Results of regression analysis.

Effect of detergents and wind on the reaeration coefficient

Conclusions and recommendations.

Conclusions.

Recommendations..

References

Appendix A : Effect of a corrosion reaction on reaeration-coefficient measurements in a laboratory flume.

Symbols used in appendix A.

Appendix B: Collected data.
Page

30

31

34

39

42

43

44

44

44

\section{ILLUSTRATIONS}

[All illustrations are graphs]

Figure 1. Example of a DO deficit sag curve

2. Computation of reaeration coefficient from recirculating-flume data..

3-10. Dissolved-oxygen balance technique:

3. Sensitivity of reaeration coefficient to rate constant for BOD decay.

4. Sensitivity of reaeration coefficient to photosynthetic-oxygen production rate

5. Sensitivity of reaeration coefficient to upstream DO deficit.

6. Sensitivity of reaeration coefficient to upstream BOD

7. Sensitivity of reaeration coefficient to traveltime...

8. Sensitivity of reaeration coefficient to downstream DO deficit

9. Effect of a systematic error in DO measurement procedure on calculated reaeration coefficient.

10. Effect of ignoring photosynthetic-oxygen production rate. 
FIgUREs 11-16. Disturbed-equilibrium technique:

11. Sensitivity of reaeration coefficient to photosynthetic-oxygen production rate................................. 39

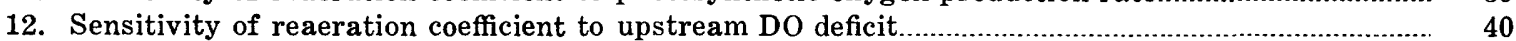

13. Sensitivity of reaeration coefficient to downstream DO deficit.................................................. 40

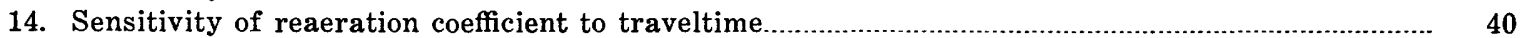

15. Effect on calculated reaeration coefficient of ignoring a respiration rate difference......................

16. Variation of calculated reaeration coefficient with respiration-rate ratio....................................... 41

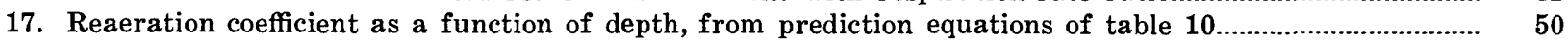

18. Synthetic flume data from the upstream sampling station.................................................................

19. Determination of $b_{1}$ and $K_{2}$ from $M$ values calculated at two different DO deficits..................................

\section{TABLES}

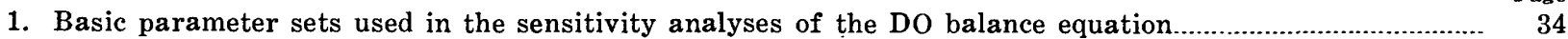

2. Assumed errors in measurements of DO balance parameter.

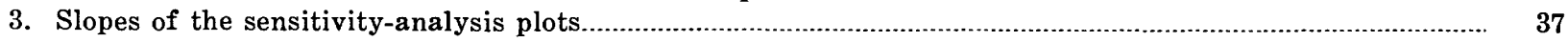

4. Errors in the calculated reaeration coefficient resulting from errors in parameter measurement, DO balance technique.

5. Basic parameter sets for the sensitivity analysis of the disturbed-equilibrium technique

6. Assumed parameter errors and slopes of sensitivity-analysis plots for disturbed-equilibrium technique

7. Errors in the calculated reaeration coefficient resulting from errors in parameter measurement, disturbed-equilibrium technique.

8. Statistical data concerning measured gas-transfer coefficient

9. Ranges of experimental data from the literature

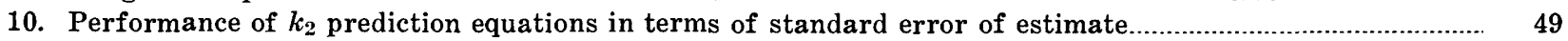

11. Results from regression analysis.

12. Values of Student $t$ for comparison of the exponents in equation 162 with those for the two corresponding field and flume equations of table 11.

13. Dependence of turbulent intensity on the mean hydraulic parameters.

14. Results of the Student $t$ test of the exponents in the flume equations and in the field equations of table 13 .

15. Prediction equations for mean velocity, obtained from the data used in table 11

16. Results of the Student $t$ test of exponents in the regression equations of this study and corresponding equations in the literature.

17. Parameters used in calculating the synthetic flume data and the resulting calculated reaeration coefficient.

18-26. Reaeration coefficients and hydraulic data from:

18. Churchill, Elmore, and Buckingham (1962)

19. Owens, Edwards, and Gibbs (1964)

20. Gameson, Truesdale, and Downing (1955)

21. O'Connor and Dobbins (1958) (Streeter and Phelps, 1925)

22. O'Connor and Dobbins (1958) (various sources)

23. Tsivoglou and others $(1967,1968)$

24. Thackston (1966)

25. Krenkel (1960)

26. Negulescu and Rojanski (1969)

\section{SYMBOLS}

Parameter in the surface-renewal-damped-eddy diffusivity model (King, 1966).

Constant in the equation for consumption of dissolved oxygen by bottom deposits.

Constant in the Edwards and Rolley (1965) equation for rate of consumption of dissolved oxygen by bottom deposits.

Rate of addition of dissolved oxygen to the reach by drainage accrual, in milligrams per liter per day.

Temperature relation in the equation of Dobbins (1965); $A=9.68+0.054\left(T-20^{\circ}\right)$, where $T$ is temperature, in degrees Celsius.

$A_{0}, A_{1} \ldots A_{N} \quad$ Coefficients in the linear-regression equation. 
$\begin{array}{ll}\text { Symbol } & \text { Definition } \\ A_{e} & \text { Parameter in the large-eddy model (Fortescue and Pear }\end{array}$

$A_{0}$

$A_{s}$

$A_{x}$

b

$b_{1}$

B

$B_{i}$

$B_{F}$

BOD

BPS

c

$c_{1}, c_{2}, c_{3}, c_{4}, c_{5}$

$c_{1}^{\prime}, c_{3}^{\prime}, c_{5}^{\prime}$

C

$\bar{C}$

$\bar{C}_{1}, \bar{C}_{2}$

$C_{\mathrm{a}}, C_{\mathrm{b}}$

$C_{\mathrm{a}}^{\prime}, C_{\mathrm{b}}^{\prime}$

$C_{i}$

$C_{d i}, C_{d f}$

$C_{i i}, C_{l f}$

$C_{s}$

$C_{s_{1}}, C_{s_{2}}$

$\bar{C}_{s}$

C

$C_{G}^{*}$

$C_{L}$

$C^{*}$

$\Delta C$

$\Delta C_{1}$

$d_{1}, d_{2}$

$d_{0}$

$d_{M}$

D

$D_{\text {a }}$

$D_{\text {a }}^{\prime}$

$D_{\mathrm{b}}$

$D_{\mathrm{b}}^{\prime}$

$D_{i}$

$D_{m}$

$D_{0}$

$D_{x}$

$D_{k}$

$D_{M}, D_{E}$

Parameter in the large-eddy model (Fortescue and Pearson, 1967), in centimeters per second.

Rate of addition of dissolved oxygen to the reach by drainage accrual, in grams per square meter per hour.

Water surface area, in square meters.

Cross-sectional area of the reach at longitudinal position $x$, in square feet.

Proportion of available molecules in the surface layer that actually escape.

Exponent on dissolved-oxygen concentration in the Edwards and Rolley (1965) equation for rate of consumption of dissolved oxygen by bottom deposits.

Temperature relation in the equation of Dobbins $(1965) ; B=0.976+0.0137\left(30^{\circ}-T\right)^{3 / 2}$, where $T$ is temperature, in degrees Celsius.

Population regression coefficient.

Factor in the Dobbins (1965) equation, equal to $1.0+\mathbf{F}^{2}$, where $\mathbf{F}$ is the Froude number.

Biochemical oxygen demand, in milligrams per liter.

Basic parameter set; a set of values assumed for the parameters of the dissolved-oxygen balance equation or the disturbed-equilibrium technique equation.

Concentration fluctuation, or the difference between the instantaneous concentration and the time-averaged concentration, in milligrams per liter or in grams per cubic meter.

Constants in the equations of Dobbins $(1964 \mathrm{~b}, 1965)$.

Constants in the Metzger-Dobbins (1967) model.

Dissolved-oxygen concentration, in milligrams per liter or in grams per cubic meter.

Mean concentration of dissolved oxygen in the reach, in milligrams per liter.

Mean concentrations of dissolved oxygen in the reach for the two levels of dissolved-oxygen concentration, disturbed-equilibrium technique, in milligrams per liter.

Dissolved-oxygen concentrations at the upstream and downstream ends of the reach, respectively, at the first level of dissolved-oxygen concentration of the disturbed-equilibrium technique, in milligrams per liter.

Dissolved-oxygen concentration at the upstream and downstream ends of reach, respectively, at the second level of dissolved-oxygen concentration of the disturbed-equilibrium technique, in milligrams per liter.

Dissolved-oxygen concentration at the gas-liquid interface, in milligrams per liter.

Initial and final concentrations, respectively, of dissolved oxygen in the dark bottle of the light- and dark-bottle test for photosynthesis, in milligrams per liter.

Initial and final concentrations, respectively, of dissolved oxygen in the light bottle of the light-and dark-bottle test for photosynthesis, in milligrams per liter.

Dissolved-oxygen concentration at saturation, in milligrams per liter or in grams per cubic meter.

Saturation concentrations of dissolved oxygen for the two levels of dissolved-oxygen concentration of the disturbedequilibrium technique, in milligrams per liter.

Mean saturation concentration of dissolved oxygen in the reach, in milligrams per liter.

Dissolved-oxygen concentration, in milligrams per liter; subscript $t$ indicates variability with respect to time at a particular point in space.

Concentration of the tracer gas in the water, in milligrams per liter.

Dissolved-oxygen concentration in the bulk liquid phase, in milligrams per liter.

Concentration of the conservative liquid tracer in the water, in milligrams per liter.

Difference in dissolved-oxygen concentration between upstream and downstream ends of the reach, in milligrams per liter.

Difference in dissolved-oxygen concentration between upstream and downstream ends of the reach at sunrise, in milligrams per liter.

Molecular diameters of gases 1 and 2, in angstroms.

Rate of transfer of oxygen through the water surface, in milligrams per liter per day.

Depth of bottom deposit, in centimeters.

Dissolved-oxygen deficit, or the difference between the saturation concentration and the concentration of dissolved oxygen, in milligrams per liter.

Dissolved-oxygen deficit at upstream end of reach, in milligrams per liter.

Dissolved-oxygen deficit at upstream end of reach at the second level of dissolved-oxygen concentration of the disturbed-equilibrium technique, in milligrams per liter.

Dissolved-oxygen deficit at downstream end of reach, in milligrams per liter.

Dissolved-oxygen deficit at downstream end of reach at the second level of dissolved-oxygen concentration of the disturbed-equilibrium technique, in milligrams per liter.

Combination of the molecular- and turbulent-diffusion coefficients at the surface, Krenkel and Orlob (1963) model, in square feet per day.

Molecular-diffusion coefficient, in square feet per day.

Rate of transfer of oxygen through the water surface, in grams per square meter per hour.

Longitudinal dispersion coefficient, in square feet per day.

Parameter that includes the effect of photosynthesis, plant respiration, and removal of dissolved oxygen by benthal layer; in milligrams per liter per day.

Average dissolved-oxygen deficit in the reach at that time in the morning and evening, respectively, when the production of oxygen by photosynthesis is zero, in milligrams per liter. 
Symbol Definition

DO Dissolved oxygen.

E

$\frac{\boldsymbol{E}^{\prime}}{\bar{E}}$

Rate of energy dissipation per unit mass of liquid, equal to $U S g$ for open-channel flow, in ergs per second per gram.

Combination of the activation energies for molecular diffusion and viscous flow, in degrees Kelvin.

Average kinetic energy of a roll cell, large-eddy model (Fortescue and Pearson, 1967), in square centimeters per second per second.

$E_{a}$

$E_{P}$

$E_{S}$

$E_{S L}$

$E_{V}$

$E_{\xi}$

$f$

$f\left(k_{2}\right)$

$f^{\prime}\left(k_{2}\right)$

$f_{1}(s)$

$f_{2}(H)$

F

$F_{c}$

$\bar{F}_{c}$

F

$g$

G

h

H

$H^{\prime}$

j

J

$k_{1}$

$k_{2}$

$\left(k_{2}\right)_{c}$

$\left(k_{2}\right)_{e}$

$k_{3}$

$k_{L}$

$K_{1}$

$K_{2}$

$\mathrm{K}_{2} \mathrm{G}$

$K_{2}{ }_{T}$

$K_{20}$

$K_{3}$

$K_{G}$

$K_{L}$

$K_{N}$

L

$L_{\mathrm{a}}$

$L_{\mathrm{b}}$

$L_{f}$

$L_{f}$

$L_{t}$

$L_{R}$

Activation energy for the absorption of oxygen by water, in calories per gram mole.

Percent standard error of estimate for the reaeration coefficient, $k_{2}$, in percent.

Standard error of estimate for the reaeration coefficient, $k_{2}$, in reciprocal days.

Standard error of estimate for the reaeration coefficient, $k_{2}$, based on common logarithms.

Rate of energy dissipation per unit volume of liquid, in ergs per second per cubic centimeter.

Rate of energy dissipation per unit mass of liquid at the surface, in ergs per second per gram.

Dissolved-oxygen exchange coefficient, in meters per hour.

Functional relation of the reaeration coefficient, common logarithm base; used in the sensitivity analyses of the dissolved-oxygen balance and disturbed-equilibrium techniques.

First derivative of the functional relation of the reaeration coefficient, common logarithm base; used in the NewtonRaphson iterative method for solving the dissolved-oxygen balance and disturbed-equilibrium equations for the sensitivity analyses.

Rate-of-surface-renewal function, Thackston and Krenkel (1969a) model.

Depth-of-flow function, Thackston and Krenkel (1969a) model.

Volumetric water flow rate, in cubic meters per hour or cubic feet per second.

Source-sink term, in milligrams per liter per day.

Source-sink term averaged over the depth of flow, in milligrams per liter per day.

Froude number, equal to $U / \sqrt{g H}$.

Acceleration of gravity, in feet per second per second.

General functional relation between dependent and independent variables in linear regression analysis.

Henry's Law constant, in liters per milligram.

Mean depth of flow, in feet or meters.

Depth above minimum low-water stage, Streeter and Phelps (1925) prediction equation for the reaeration coefficient, in feet.

Exponent on depth of flow in the general form of the empirical prediction equation for the reaeration coefficient.

Constant in the Thackston and Krenkel (1969a) model.

Deoxygenation coefficient or rate constant for biochemical oxidation of carbonaceous material, common logarithm base, in reciprocal days.

Reaeration coefficient or rate constant for oxygen absorption from the atmosphere, common logarithm base, in reciprocal days, or in reciprocal minutes.

Reaeration coefficient calculated from the results of an experimental test, common logarithm base, in reciprocal days.

Reaeration coefficient estimated from a linear regression analysis equation, common logarithm base, in reciprocal days.

Rate constant for the removal of BOD by sedimentation and adsorption, common logarithm base, in reciprocal days. Overall mass-transfer coefficient based on liquid phase, equivalent to $k_{2} H$, in centimeters per second or in feet per day.

Deoxygenation coefficient or rate constant for biochemical oxidation of carbonaceous material, natural logarithm base, in reciprocal days.

Reaeration coefficient or rate constant for oxygen absorption from the atmosphere, natural logarithm base, in reciprocal days.

Mass-transfer coefficient for the tracer gas, in reciprocal days.

Reaeration coefficient at $T^{\circ}$ Celsius, in reciprocal days.

Reaeration coefficient at $20^{\circ}$ Celsius, in reciprocal days.

Rate constant for the removal of BOD by sedimentation and adsorption, in reciprocal days.

Overall mass-transfer coefficient based on the gas phase, in milligrams per square centimeter per second per atmosphere.

Overall mass-transfer coefficient based on the liquid phase, in centimeters per second or in feet per day.

Rate constant for the oxidation of nitrogenous material, in reciprocal days.

Biochemical oxygen demand of carbonaceous material, in milligrams per liter.

Biochemical oxygen demand of carbonaceous material at upstream end of reach, in milligrams per liter.

Biochemical oxygen demand of carbonaceous material at downstream end of the reach, in milligrams per liter.

Film thickness, in feet or meters.

Average film thickness, in feet or meters.

Biochemical oxygen demand of carbonaceous material, in milligrams per liter; subscript $t$ indicates variability with respect to time at a particular point in space.

Rate of addition of BOD along the reach, in milligrams per liter per day. (Includes addition of BOD with runoff, the diffusion of partially degraded waste from the benthal layer into the water, and the scour and resuspension of bottom deposits.) 
$R_{G}$

$R_{H}$

$R_{M}$

$R_{P}$

Definition

Rate of addition of BOD to water from bottom deposits, in milligrams per liter per day.

Molecular weight.

Modulus of compression at the surface, in grams per second per second.

An integer.

Number of new surface layers exposed per unit of time, in reciprocal seconds.

Mass-transport rate of oxygen, in milligrams per square centimeter per second.

Average rate of mass transport of oxygen, in milligrams per square centimeter per second.

Number of data sets in linear regression analysis.

Concentration of nitrogenous material at longitudinal position $x$, in milligrams per liter.

Rate of production of dissolved oxygen by photosynthesis, in milligrams per liter per day.

Rate of production of dissolved oxygen by photosynthesis at the second level of dissolved-oxygen concentration of the disturbed-equilibrium technique, in milligrams per liter per day.

Distribution function for surface-renewal time or for distance below the interface to which renewal occurs $(f$ is dummy variable for either $t$ or $y_{1}$ ), Harriott (1962) model.

Partial pressure of the diffusing gas, oxygen, at the gas-liquid interface, in atmospheres.

Rate of production of dissolved oxygen by photosynthesis, in milligrams per liter per day; subscript $t$ indicates variability with respect to time at a particular point in space.

Rate of production of dissolved oxygen by photosynthesis at longitudinal position $x$ at time $t$, in milligrams per liter per day.

Barometric pressure, in atmospheres.

Partial pressure of the diffusing gas, oxygen, in the gas phase, in atmospheres.

Rate of gross primary production of dissolved oxygen by photosynthesis, in grams per square meter per hour.

Rate of production of dissolved oxygen by photosynthesis in the light bottle of the light- and dark-bottle test, in milligrams per liter per day.

Gross rate of photosynthetic production of dissolved oxygen by attached plants, in grams per square meter per hour. Gross rate of photosynthetic production of dissolved oxygen by phytoplankton, in grams per square meter per hour.

Peclet number, equal to $\Lambda A_{e} / D_{m}$.

Rate of change of dissolved-oxygen content in the reach, in milligrams per liter per day.

Rate of change of dissolved-oxygen content in the reach at sunrise, in milligrams per liter per day.

Rate of change of dissolved-oxygen content in the reach at that time in the morning and evening, respectively, when the production of oxygen by photosynthesis is zero, in milligrams per liter per day.

Rate of change of dissolved-oxygen content in the reach, in grams per square meter per hour.

Average rate of change of dissolved-oxygen concentration per unit area in the reach for the two levels of dissolvedoxygen concentration, disturbed-equilibrium technique, in grams per square meter per hour.

Rate of consumption of dissolved oxygen by plant respiration, in milligrams per liter per day.

Rate of entry of gas molecules into the surface layer, in grams per second.

Rate of loss of gas molecules from a surface layer, in grams per second.

Rate of consumption of dissolved oxygen by plant respiration, in milligrams per liter per day; subscript $t$ indicates variability with respect to time at a particular point in space.

Rate of consumption of dissolved oxygen by plant respiration at longitudinal position $x$ at time $t$, in milligrams per liter per day.

Rate of dissolved-oxygen utilization by all processes, in grams per square meter per hour.

Ratio of the oxygen consumed by respiration at the two levels of dissolved-oxygen concentration, disturbedequilibrium technique.

Rate of consumption of dissolved oxygen by respiration in the dark and light bottles, respectively, of the light- and dark-bottle test for photosynthesis, in milligrams per liter per day.

Equals the quantity $\left(A_{i}-B_{i}\right) / S_{b i}$, used to test the significance of the regression coefficient $A_{i}$.

Rate of consumption of dissolved oxygen by respiration by attached plants, in grams per square meter per hour.

Rate of consumption of dissolved oxygen by the oxidation of organic wastes in the stream water, in grams per square meter per hour.

Ideal-gas constant, in calories per gram mole per degree Kelvin.

Hydraulic radius, equal to the cross-sectional area of flow divided by the wetted perimeter, in feet.

Rate of consumption of dissolved oxygen by bottom deposits, in grams per square meter per hour.

Rate of consumption of dissolved oxygen by respiration of phytoplankton, in grams per square meter per hour.

Root-mean-square, or square root of the mean value of the square of the deviations of the measured parameter from the mean parameter.

Average rate of surface renewal, in reciprocal hours.

Water-surface or channel slope, in feet per foot.

Standard error of the regression coefficient $A_{i}$.

Rate of removal of BOD by sedimentation at longitudinal position $x$ at time $t$, in milligrams per liter per day.

Time, in days or hours.

Flow time, or the time that must elapse between dissolved-oxygen measurements at the two sampling points in a recirculating flume, in seconds. 


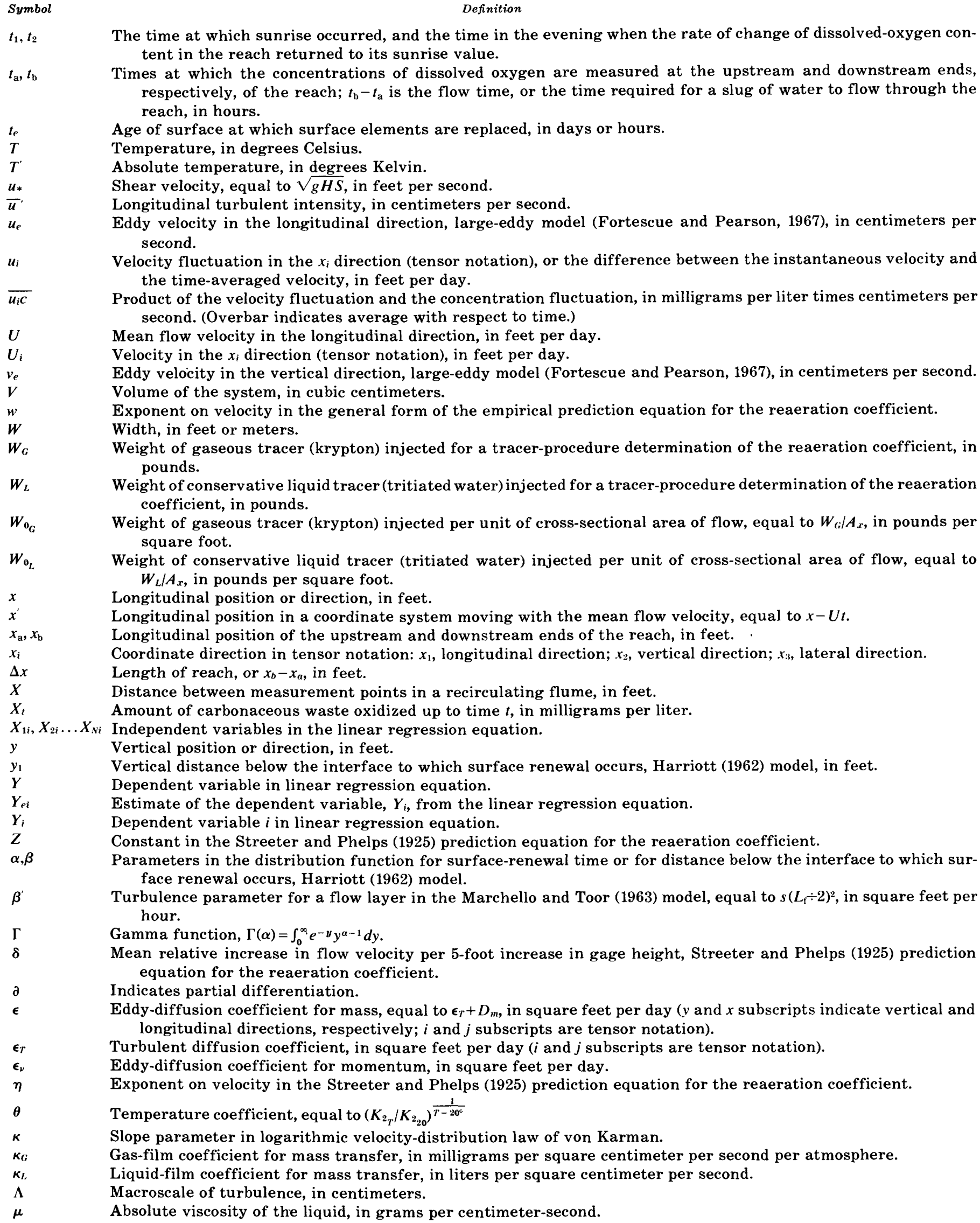
hour.

Gamma function, $\Gamma(\alpha)=\int_{0}^{\infty} e^{-y} y^{\alpha-1} d y$.

Mean relative increase in flow velocity per 5-foot increase in gage height, Streeter and Phelps (1925) prediction equation for the reaeration coefficient.

Indicates partial differentiation.

Eddy-diffusion coefficient for mass, equal to $\epsilon_{T}+D_{m}$, in square feet per day $(y$ and $x$ subscripts indicate vertical and longitudinal directions, respectively; $i$ and $j$ subscripts are tensor notation).

Turbulent diffusion coefficient, in square feet per day ( $i$ and $j$ subscripts are tensor notation).

Eddy-diffusion coefficient for momentum, in square feet per day.

Exponent on velocity in the Streeter and Phelps (1925) prediction equation for the reaeration coefficient.

Temperature coefficient, equal to $\left(K_{2_{T}} / K_{2_{20}}\right)^{\frac{1}{T-20^{\circ}}}$

Slope parameter in logarithmic velocity-distribution law of von Karman.

Gas-film coefficient for mass transfer, in milligrams per square centimeter per second per atmosphere.

Liquid-film coefficient for mass transfer, in liters per square centimeter per second.

Macroscale of turbulence, in centimeters.

Absolute viscosity of the liquid, in grams per centimeter-second. 
Velocity normal to the interface that a gas molecule must have to be able to leave the liquid, in centimeters per second.

Kinematic viscosity of the liquid, in square centimeters per second.

The constant 3.1416.

Mass density of the liquid, in grams per cubic centimeter.

Surface tension of the liquid, in dynes per centimeter.

Distribution function for surface ages.

Constant in the Isaacs and Gaudy (1968) equation.

Constant in the empirical prediction equation for the reaeration coefficient.

Dissolved-oxygen consumption rate by bottom deposits, in grams per square meter per hour. 



\title{
REAERATION IN OPEN-CHANNEL FLOW
}

\author{
By J. P. BENNETT and R. E. RATHBun
}

\section{ABSTRACT}

Reaeration, the physical absorption of oxygen from the atmosphere, is the primary process by which a stream replaces oxygen consumed in the biodegradation of organic wastes. The reaeration process in a stream is characterized by its surface reaeration coefficient. Hence, knowledge of the reaeration coefficient permits determination of the quantity of waste that can be discharged into a stream without causing serious depletion of the dissolved-oxygen content of the stream.

The three basic methods for measuring the reaeration coefficient are the dissolved-oxygen balance, disturbed-equilibrium, and tracer procedures. The dissolved-oxygen balance method consists of measuring the various sources and sinks of dissolved oxygen and determining by difference the amount of reaeration needed to balance the equation. The disturbedequilibrium method consists of artificially producing dissolvedoxygen deficits by adding sodium sulfite to the stream and subsequently measuring upstream and downstream concentrations of dissolved oxygen at two different concentration levels. The tracer method consists of using an inert radioactive gas as a tracer for oxygen and correlating the rate of desorption of the tracer gas with the rate of absorption of oxygen.

Various theoretical models of the oxygen-absorption process exist; however, these models are generally not suited for prediction of the reaeration coefficient in streams because the model parameters have not been adequately related to bulkflow hydraulic variables. Semiempirical and empirical equations developed from experimental data adequately predict reaeration coefficients for streams of the type on which the equations were based, but large errors may occur when the equations are applied to other types of streams or to conditions outside the range of variables considered in the original correlation.

\section{INTRODUCTION}

Rivers and streams are used for the disposal of municipal and industrial wastes, and Hull (1963) suggested that this use is one of the most important factors contributing to the general health and welfare of the people of the United States. Discharge of wastes into a stream, however, does not simply dilute the wastes. Each stream and river has a natural capacity for oxidizing biodegradable wastes, thus purifying the waters. This purification capacity is dependent upon many factors, including the water discharge, the depth of flow, the velocity of flow, and the various sources and sinks of dissolved oxygen along the stream. The natural purification capacity of a stream therefore will vary as the hydraulic conditions in the stream change with time.

Although the flow in a specific stream may have varied greatly from day to day and from year to year, the total annual runoff for the United States as a whole showed only a small, apparently cyclic, variation with time for the years 1895 to 1955 (Leopold and Langbein, 1960). On the other hand, the population and the number of industrial facilities have increased tremendously, and this growth has placed an ever increasing burden on the rivers and streams of the United States. In many instances, the purification capacities of streams and rivers have been exceeded.

The fact that municipal and industrial wastes are discharged into a stream does not necessarily mean that the stream is polluted, because the stream does have the capacity for self-purification. The definition of pollution has received considerable attention. The Colorado Supreme Court in 1934 (Gindler, 1967, p. 5) defined pollution as follows:

For the purpose of this case, the word "pollution" means an impairment, with attendant injury, to the use of water that the plaintiffs are entitled to make. Unless the introduction of extraneous matter so unfavorably affects such use, the condition is short of pollution. In reality, the thing forbidden is injury. The quantity introduced is immaterial.

Other definitions were discussed by Haney (1966), and he proposed (p. 110) that "pollution is the impairment of water quality, with resultant significant interference with beneficial water use." Apparently, from these definitions, the determination of when pollution exists depends both on the intended use of the stream and on some water quality parameter that will insure that the water is of a quality adequate for the intended purpose.

Of the various indicators of water quality (such as the coliform bacteria count, the hydrogen ion concentration, and the concentrations of phosphates, nitrates, synthetic organics, and industrial chemicals), the one most often used as a measure of pollution by biodegradable organic wastes is DO (dissolved oxygen) concentration (Wolman, 
1960). This report uses DO concentration as the principal measure of water quality. Thus the condition of a stream is determined from a DO balance which includes all the sources and sinks of DO along the reach of interest. The various types of DO balances for streams and rivers that have appeared in the literature are discussed in the following paragraphs.

The purification capacity of a stream depends upon its DO resources and its ability to replace the oxygen consumed in the oxidation of organic wastes. One of the first studies of the DO balance was the classical study of Streeter and Phelps (1925). These authors assumed one sink and one source of DO.

The oxygen sink was the biochemical oxidation of carbonaceous wastes by bacteria in the stream. It is generally assumed that biochemical oxidation is a first-order process; that is, the rate of biochemical oxidation is proportional to the amount of carbonaceous material remaining to be oxidized. This process may be described by

$$
\frac{d L}{d t}=-K_{1} L
$$

where $L$ is the BOD (biochemical oxygen demand) of the carbonaceous material, $t$ is time, and $K_{1}$ is the deoxygenation coefficient or the rate constant for the oxidation of carbonaceous wastes. Note that the concentration of biodegradable waste is expressed in terms of the oxygen demand. The concentration of biodegradable waste in the stream is not directly measurable and is of importance only because it controls the demand for DO. The DO demand is determined by a BOD test, and standard procedures for the test were described by the American Public Health Association (1965). If the DO concentration is expressed in terms of the saturation deficit, $D$, or the difference between the DO saturation concentration and the actual concentration at time $t$, then

$$
\frac{d D}{d t}=-\frac{d L}{d t}
$$

The oxygen source considered by Streeter and Phelps (1925) was reaeration, or the physical absorption of oxygen from the atmosphere by the flowing stream. Adeney and Becker (1919, 1920) showed that the absorption of oxygen by water is a first-order process; that is, the rate of absorption is directly proportional to the saturation deficit. This process may be described by

$$
\frac{d D}{d t}=-K_{2} D,
$$

where $K_{2}$ is the reaeration coefficient or the rate constant for the absorption of oxygen from the atmosphere and $D$ is the DO deficit.
Streeter and Phelps (1925) assumed that the DO deficit was determined by these two independent processes. Therefore, the DO balance was

$$
\frac{d D}{d t}=K_{1} L-K_{2} D \text {. }
$$

The solution of equation 1 is

$$
L=L_{\mathrm{a}} \exp \left(-K_{1} t\right) \text {. }
$$

Substituting equation 5 in equation 4 , the solution of equation 4 is

$D=\frac{K_{1} L_{\mathrm{a}}}{K_{2}-K_{1}}\left[\exp \left(-K_{1} t\right)-\exp \left(-K_{2} t\right)\right]+D_{\mathrm{a}} \exp \left(-K_{2} t\right)$,

where $L_{\mathrm{a}}$ is the BOD of the carbonaceous material and $D_{\mathrm{a}}$ is the DO deficit just upstream from the point at which the waste is added to the stream. The time, $t$, is expressed in terms of flow time along the stream. If $t$ is flow time, then $d D / d t$ is equivalent to $U d D / d x$, where $U$ is the mean flow velocity, in feet per day, and $x$ is longitudinal distance, in feet. The factors $D$ and $L$ are usually expressed in parts per million or milligrams per liter, and $K_{1}$ and $K_{2}$ are usually expressed in reciprocal days. A plot of $D$ as a function of $t$ gives the classical sag curve for DO deficit. An assumption inherent in equation 6 is that the initial pollution load is added at the upstream end of the reach and thereafter is affected only by biochemical oxidation and not by the addition of either pollution or dilution flow along the reach.

Streeter and Phelps (1925) were aware that the assumption of a slug injection of BOD was an oversimplification of the actual situation; hence, they also solved equation 4 for a BOD source uniformly distributed along the reach of interest. This BOD distribution resulted in a much more complicated solution. Other assumptions regarding the distribution of the BOD load are possible, and the complexity of the solution, equation 6 , increases as the complexity of the assumed distribution for $L$ increases.

An example of an oxygen sag curve for an $L_{\mathrm{a}}$ of $9.0 \mathrm{mg} / \mathrm{l}$ (milligrams per liter), a $D_{\mathrm{a}}$ of $1.0 \mathrm{mg} / \mathrm{l}$, a $K_{1}$ of 0.1 days $^{-1}$ (reciprocal days), and a $K_{2}$ of 0.2 days $^{-1}$ is presented in figure 1. Just below the point of addition of the pollution load, the oxidation of the waste predominates, and the DO deficit increases. However, reaeration begins as soon as a DO deficit develops, and the rate of reaeration increases as the deficit increases. Finally, at the minimum deficit of the oxygen sag curve, the rate of supply of oxygen by reaeration equals the rate of consumption of oxygen by biochemical oxidation. Thereafter, the reaeration process predominates, and the DO deficit is gradually reduced 


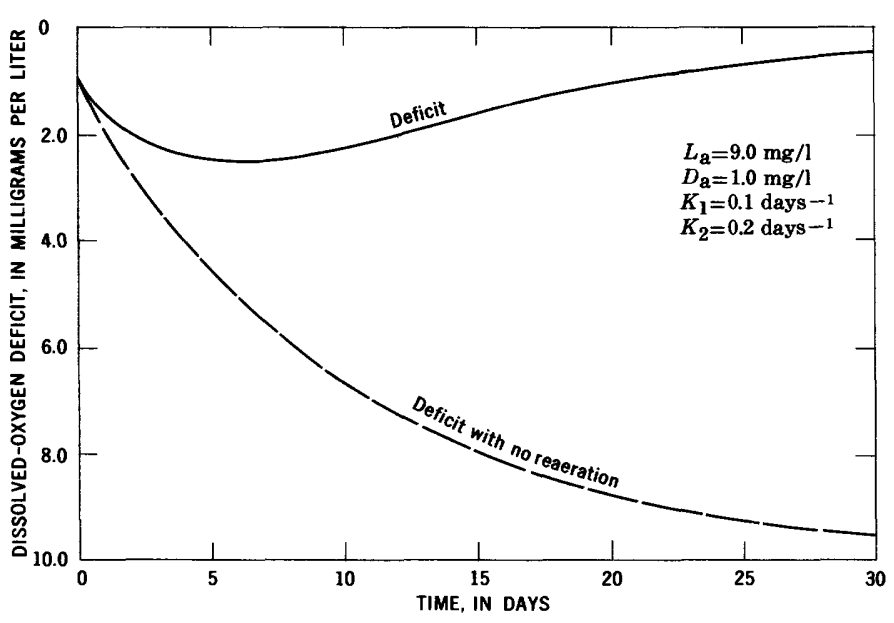

Figure 1. - Example of a DO deficit sag curve.

to zero. Also shown in figure 1 is the increase in the DO deficit in the absence of reaeration $\left(K_{2}=0\right)$.

The oxygen sag equation of Streeter and Phelps (1925) has been widely used, and different forms of the equation have appeared in the literature. Some authors have used common logarithms (base 10 ), and the deoxygenation and reaeration coefficients under these circumstances are generally represented by $k_{1}$ and $k_{2}$, respectively, where

$$
K_{1}=2.30 k_{1}
$$

and

$$
K_{2}=2.30 K_{2} \text {. }
$$

A mathematical analysis of the Streeter-Phelps equations was presented by Fair (1939).

Processes other than biochemical oxidation and reaeration also may be important in the DO balance of a stream. Although Streeter and Phelps (1925) chose to include only these two fundamental processes in their oxygen balance, they were aware of the possible effects of inflow along the reach, sedimentation and scouring in the reach, and photosynthesis (Streeter and Phelps, 1925, p. 10, 22). Other authors, notably Dobbins (1964b) and Camp (1963), have expanded the fundamental Streeter-Phelps equation to include in the oxygen balance these and other sources and sinks of DO.

Dobbins (1964b) included the removal of BOD by sedimentation or adsorption on bottom sediments, the removal of DO from the water by diffusion into the benthal layer and by plankton and fixed-plant respiration, the addition of $\mathrm{BOD}$ along the reach by runoff and by scour of bottom deposits, the addition of DO by the photosynthetic process of plankton and fixed plants, and the continuous redistribution of the $\mathrm{BOD}$ and $\mathrm{DO}$ by the effects of longitudinal dispersion.
With these sources and sinks considered, the DO balance has the form

$$
\frac{\partial C}{\partial t}+U \frac{\partial C}{\partial x}=D_{x} \frac{\partial^{2} C}{\partial x^{2}}+K_{2}\left(C_{s}-C\right)-K_{1} L-D_{B},
$$

where $C$ is the DO concentration, $C_{s}$ is the saturation DO concentration, $x$ is longitudinal distance along the reach, $U$ is the mean stream velocity, $D_{x}$ is the longitudinal-dispersion coefficient, and $D_{B}$ includes the removal of DO by diffusion into the benthal layer and the effect of photosynthesis and respiration by plants. Therefore, the algebraic value of $D_{B}$ may be positive or negative, depending on the relative magnitudes of the three processes. Dobbins (1964b) used the convention that $D_{B}$ is positive when the rate of removal of DO exceeds the rate of addition.

A BOD balance analogous to the DO balance may be written as follows:

$$
\frac{\partial L}{\partial t}+U \frac{\partial L}{\partial x}=D_{x} \frac{\partial^{2} L}{\partial x^{2}}-\left(K_{1}+K_{3}\right) L+L_{R},
$$

where $K_{3}$ is the rate constant for the removal of BOD by sedimentation and adsorption, and $L_{R}$ is the rate of addition of $\mathrm{BOD}$ along the reach, including the addition of BOD with runoff, the diffusion of partially degraded waste from the benthal layer into the water, and the scour and resuspension of bottom deposits.

In general, steady-state and uniform-flow conditions are assumed in a DO balance study. With these assumptions, the $\partial C / \partial t$ and $\partial L / \partial t$ terms are zero, and the water discharge, the input BOD load, the temperature, and the rate constants at each point in the reach are assumed independent of time. Other assumptions inherent in equations 9 and 10 are as follows:

1. The DO and BOD are uniformly distributed over each cross section so that equations 9 and 10 can be written in the one-dimensional form.

2. The processes described by the rate constants $K_{1}, K_{2}$, and $K_{3}$ are first-order processes; that is, the rate of removal of BOD is proportional to the amount of $\mathrm{BOD}$ remaining, and the rate of reaeration is proportional to the $\mathrm{DO}$ deficit.

3. The processes represented by $L_{R}$ and $D_{B}$ are uniformly distributed along the reach.

4. Only the carbonaceous demand of the waste is significant. If the nitrogenous demand is important, an additional term must be added to equations 9 and 10.

Each of the terms in equations 9 and 10 represents a time-rate of change of the BOD or DO concentration, and the units used are generally 
parts per million per day or milligrams per liter per day.

Dobbins (1964b) solved equations 9 and 10 using these assumptions and the added assumption that the effect of longitudinal dispersion is negligible compared with the other terms for natural streams. For the BOD equation, the solution showed that the BOD may either decrease or increase along the reach, depending on whether $L_{\mathrm{a}}$ is greater or less than $L_{R} /\left(K_{1}+K_{3}\right)$, where $L_{\mathrm{a}}$ is the initial BOD load at the upstream end of the reach. Dobbins (1964b) suggested a procedure for the evaluation of the various constants, and he also discussed the $D_{B}$ term, which includes several different processes.

Camp (1963) presented DO and BOD balance equations similar in form to equations 9 and 10. Primary differences are in the use of $d C / d t$ and $d L / d t$ as opposed to $U d C / d x$ and $U d L / d x$ and in the treatment of the $D_{B}$ term. With the assumptions of steady-state and uniform-flow conditions,

$$
\frac{d}{d t}=U \frac{d}{d x}
$$

Therefore, Camp's equations (1963, p. 295, 297) may be written as

$$
\frac{d C}{d t}=K_{2}\left(C_{s}-C\right)-K_{1} L+p
$$

for the DO balance and

$$
\frac{d L}{d t}=-\left(K_{1}+K_{3}\right) L+m
$$

for the BOD balance. In equation $12, p$ is the rate of production of oxygen by photosynthesis; and in equation 13, $m$ is the rate of addition of BOD to the water from bottom deposits. For streams where longitudinal mixing is important, Camp (1963, p. 311) included a $D_{x}\left(d^{2} / d x^{2}\right)$ term on the righthand side of equations 12 and 13.

Other forms of $\mathrm{DO}$ balances have been used. The Water Pollution Research Laboratory group at Stevenage, England (Edwards and Owens, 1962; Edwards, 1962; Edwards and Owens, 1965; Owens, 1965), has used a DO balance which consists simply of an algebraic summation of the contributions or debits of the various sources and sinks of DO. The equation is

$$
Q_{0}=P \pm D_{0}-R,
$$

where $P$ is the rate of production of oxygen by photosynthesis, $R$ is the rate of oxygen utilization, $D_{0}$ is the rate of transfer of oxygen through the surface of the stream, and $Q_{0}$ is the rate of change of DO content in the reach of interest. Equation 14 is an integral form of the DO balance in which the various coefficients are assumed to be independent of time and longitudinal position within the reach. Stream flow is also assumed to be constant and uniform over the reach of interest.

The factor $Q_{0}$ is defined by

$$
Q_{0}=\frac{F}{A_{s}}\left(C_{\mathrm{b}}-C_{\mathrm{a}}\right),
$$

where $F$ is the volumetric rate of flow of water, $A_{s}$ is the water surface area of the reach, and $C_{\mathrm{b}}$ and $C_{\mathrm{a}}$ are the concentrations of DO at the downstream and upstream ends, respectively, of the reach at times $t_{\mathrm{b}}$ and $t_{\mathrm{a}}$, where $t_{\mathrm{b}}-t_{\mathrm{a}}$ is the time required for a slug of water to flow through the reach.

The factor $D_{0}$ is defined by

$$
D_{0}=f\left(\bar{C}_{s}-\bar{C}\right) \text {, }
$$

where $f$ is the exchange coefficient for the reach, and $\bar{C}_{s}$ and $\bar{C}$ are the mean saturation and the mean concentration of dissolved oxygen in the reach, respectively.

Each term in equations 14 to 16 has the dimensions of grams per square meter per hour, or equivalent, and is a rate per unit of surface area. To put these equations in dimensions comparable to the dimensions of the Streeter and Phelps (1925), Camp (1963), and Dobbins (1964b) equations, each term must be divided by the mean depth of flow. Thus, the exchange coefficient, $f$, divided by the mean depth of flow is equivalent to the reaeration coefficient, $K_{2}$.

The basic DO balance, equation 14, was modified as needed to meet the conditions in the stream under study. For example, Owens and Edwards (1966) used

$$
Q_{0}=P_{B}+P_{P}-R_{B}-R_{P} \pm D_{0},
$$

where the $B$ subscript refers to bottom deposits and attached plants, and the $P$ subscript refers to phytoplankton, which live unattached in the water. In the River Lark, in England, just below a sewage works, Owens and Edwards (1963) found that the consumption of oxygen by the bottom deposits or mud was a very important factor. Thus the respiration term, $R_{B}$, for bottom deposits was divided into two terms, $R_{B}$ and $R_{M}$, where the subscript $B$ refers to attached bottom plants (benthos) and the subscript $M$ refers to mud or bottom deposits. The resultant equation,

$$
Q_{0}=P_{B}+P_{P}-R_{M}-R_{B}-R_{P}+D_{0},
$$

was discussed by Owens and Knowles (1968). If significant organic pollution exists in the water of the stream, then an additional oxygen $\operatorname{sink}, R_{\mathrm{BOD}}$, must be added to the right-hand side of equation 18 .

Bain (1968) used a modified form of the StreeterPhelps equation for predicting the variation of the DO concentration with time at a point. His DO balance was 


$$
\frac{d C_{t}}{d t}=p_{t}-r_{t}-K_{1} L_{t}+K_{2}\left(C_{s}-C_{t}\right),
$$

where the $t$ subscript indicates variability with time at a particular point in space. Bain (1968) wrote equation 19 for each of six 4-hour periods, giving a set of six simultaneous equations for each day of observation. These equations were solved by a numerical technique using sequential substitution, and the result was a dynamic DO balance for a 24-hour period at a specific point in the stream.

O'Connell and Thomas (1965) considered the assumption of steady-state conditions as the major fault of the Streeter-Phelps equation and therefore used

$$
\frac{\partial C}{\partial t}+U \frac{\partial C}{\partial x}=p-r+K_{2} D-K_{1} L .
$$

They solved equation 20 using the assumption that the $p$ and $r$ factors were constant with respect to time and position. Hence, the solution is applicable only at a point for a short interval of flow time.

Li (1962) presented a generalized solution of the Streeter-Phelps equation that was expanded to include the effects of BOD and runoff additions along the stream and of fluctuations of the BOD load and DO concentration at the point of addition of the pollution to the stream. He assumed that the variations in BOD load and DO concentration were cyclic and were repeated cycle after cycle.

O'Connor (1967) wrote a general DO balance for a one-dimensional stream in which the oxygen demand of the waste is divided into the requirements for the carbonaceous material and those for the nitrogenous material. The result is

$$
\begin{aligned}
\frac{\partial C}{\partial t}+\frac{F}{A_{x}} \frac{\partial C}{\partial x}=K_{2}\left(C_{s}-C\right)+p_{x, t} & -K_{1} L \\
& -K_{N} N_{x}-r_{x, t}-S E_{x, t},
\end{aligned}
$$

where $F$ is the volumetric rate of flow, $A_{x}$ is the cross-sectional area of flow at longitudinal position $x, K_{N}$ is the rate constant for the oxidation of nitrogenous material, $N_{x}$ is the concentration of nitrogenous material at $x, S E$ is the rate of removal of BOD by sedimentation, $p$ is the rate of production of DO by photosynthesis, $r$ is the rate of consumption of dissolved oxygen by plant respiration, and the $x, t$ subscript indicates the longitudinal position $x$ at time $t$. The oxygen demand of the nitrogenous material has not received as much attention as the oxygen demand of the carbonaceous material. However, work by Courchaine (1968), O'Connell and Thomas (1965), Wezernak and Gannon (1968), and O'Connor, St. John, and DiToro (1968) has shown the importance of nitrification on the DO balance of streams receiving discharges of municipal waste.

O'Connor (1967) solved the general DO balance (eq 21) analytically using the assumptions that the photosynthetic rate varied sinusoidally with time and that the maximum rate of photosynthesis and the factors $r_{x, t}$ and $S E_{x, t}$ were independent of position and time. The solution was applicable to a sunlight period of 12 hours. O'Connor discussed in general terms two limiting forms of the solution. In the first situation the stream is heavily polluted, is shaded, and contains large amounts of suspended materials and (or) small amounts of nutrient materials. Under these conditions, $p_{x, t}$ and $r_{x, t}$ are small and are negligible with respect to the BOD factors, and the assumption of steady-state conditions is reasonably valid. The solution reduces to the oxygen sag equation of Streeter and Phelps (1925) with a term added for the oxygen demands of the bottom deposits. In the second situation, the stream is in the latter stage of self-purification. Oxidation of organic material is essentially complete, and bacterial respiration is negligible in comparison with algae respiration. The assumption of steady-state conditions is not valid, and the solution reduces to an equation that describes the temporal distribution of $\mathrm{DO}$ as a result of photosynthetic activity.

The variety of $\mathrm{DO}$ balances that have appeared in the literature demonstrates an important fact: each stream and each reach of a stream must be considered as a separate problem. Generalizations are possible, however. For example, McConnell and Sigler (1959) found in a shallow, swift stream that the effect of the stream metabolism on the DO content was negligible compared with the effect of reaeration. On the other hand, in the River Ivel, in England, where the water velocity seldom was greater than 6 centimeters per second, reaeration was relatively unimportant (Owens, 1965). Therefore, in an initial DO balance on a stream, all possible sources and sinks of DO should be considered. The unimportant factors may then be eliminated as they are shown to be negligible.

With the development of computers, the computation of DO distributions from complex analytical solutions of DO balances was simplified greatly. Frankel (1965) developed a dynamic model of the sag equation for computer solution. His model was an extension of the model of $\mathrm{Li}$ (1962) to include a variable DO source or sink caused by the presence of algae.

In addition to simplifying computations, computers made possible numerical solutions that 
previously would not have been feasible. Dresnack and Dobbins (1968) presented numerical solutions of the BOD and DO equations where BOD and DO inputs varying with time and space prevented analytical solutions. These solutions were limited to steady-state and uniform-flow conditions. Three examples were considered. These were: (1) The effect of the longitudinal-dispersion coefficient on the concentration profile of a conservative pollutant introduced at the upstream end of the reach in a periodically varying manner; (2) the effect of sinusoidal inputs of BOD and DO at the upstream end of the reach on the BOD and DO profiles; and (3) the effects of sinusoidally varying inputs of BOD and DO and a sinusoidal variation in the algae effects. Bella and Dobbins (1968) presented a modeling procedure using finite-difference techniques for BOD and DO profiles in streams and estuaries; this procedure considered temporal and spatial variations in hydraulic conditions.

The development of computers also has resulted in the advancement of the concepts of systems analysis, and these concepts in turn have been applied to the modeling of the DO resources of a stream. A system may in general be defined as an entity which operates on or transforms an input to produce an output. Thus the system consists of the input, the output, and the transformation between the two. Thomann (1963) presented a general model for describing the variations of DO with time in a finite number of sections of a body of water and applied this model (1965) to the Delaware River Estuary. He determined the transient response of the estuary to an instantaneous injection of waste, demonstrated the ease of extending the systems-analysis model to two dimensions, and determined the steady-state conditions for the one-dimensional model. He also applied the linear programing concept to the steady-state conditions to determine the most economical combination of waste treatments for the various waste sources that would assure a minimum steady-state concentration of DO of $2 \mathrm{mg} / \mathrm{l}$. The latter problem was solved for two values of the reaeration coefficient.

Liebman and Lynn (1966) applied dynamic programing in a simplified example of river-basin planning based on data from the Willamette River, Oreg. The objective was to determine the amount of BOD removal required of each waste discharger so that the water-quality standards for DO would be met with minimum total cost to the basin. The solution of the Streeter-Phelps equation was used with $K_{1}$ and $K_{2}$ assumed known and constant in each reach. The equation was modified, however, to account for changes in the saturation concen- tration, $C_{s}$, as a result of temperature changes. $C_{s}$ was assumed to vary linearly with distance downstream. Three situations were considered: (1) the configuration of treatment plants that would meet the DO standards with minimum cost; (2) same as No. 1 except that the minimum DO standard was reduced $0.1 \mathrm{mg} / 1$; and (3) same as No. 1 except the requirement was added that all plants provide the same percentage of BOD removal.

The general application of the concepts of linear programing to the solution of the DO balance equation and the determination of the degrees of treatment required for minimum basin cost were discussed by ReVelle, Loucks, and Lynn (1967). The application of linear programing to the data from the Willamette River, Oreg., was discussed by Revelle, Loucks, and Lynn (1968). The results were essentially the same as those obtained with dynamic programing.

Most DO balances give one value for the DO concentration at a downstream point. Similarly, most DO quality standards do not distinguish as to the degree or duration of a pollution violation. This consideration is important because a DO concentration that is sufficient on the average to assure healthy fish does not prevent fish kills. Thayer and Krutchkoff (1967) presented a stochastic model applicable to steady-state flow and input BOD and DO conditions that gives the probability distributions for the BOD and DO concentrations at downstream points. With the confidence limits determined from the distributions, the proportion of the time that the BOD will be greater than any specified concentration can be determined. The model can be used with any flow condition, such as the expected minimum flow condition, provided the parameters $K_{1}, K_{2}, K_{3}$, and $D_{B}$ associated with the flow condition are known.

Loucks and Lynn (1966) developed probabilistic models for predicting the probability distribution of minimum DO concentration at points downstream from a waste-treatment plant. The models were based on different mean daily stream and waste flow conditions. Loucks and Lynn suggested that the use of a maximum allowable probability of the DO concentration falling below a specified concentration for a given number of consecutive days was a more realistic approach than the deterministic DO standards generally used.

Kothandaraman and Ewing (1969) considered the effect of variations in $K_{1}$ and $K_{2}$ on the downstream DO distributions and developed procedures for taking the expected variations into account in predicting the DO. A Monte Carlo simulation technique was used to analogize the variations in 
$K_{1}$ and $K_{2}$. The variations of $K_{1}$ and $K_{2}$ within their practical ranges were found to have significant effects in the prediction of the DO concentrations, and the errors increased with temperature increase.

DiToro and O'Connor (1968) studied the effect of time-varying discharge and mean velocity on the $\mathrm{BOD}$ and DO distributions. They found that the standard deviations of tile resultant BOD and DO distributions were approximately equal to the coefficient of variation of the velocity multiplied by the BOD or DO distribution calculated using the mean velocity.

Despite the sophistication of the mathematical techniques permitted by the development of computers, the various parameters of the DO balance still must be measured or estimated. Thomann (1965) stated that his systems-analysis study of the Delaware River Estuary pointed out "the importance of good engineering evaluation of the system parameters of flow, diffusion, reaeration, and decay coefficients."

A critical analysis of previous theoretical and experimental work concerning the reaeration coefficient in open-channel flows is given on the following pages. The discussion covers theoretical and empirical models for prediction of $K_{2}$, techniques for experimental determination of $K_{2}$, an analysis of previous experimental data, a suggested prediction equation, and suggestions for future research. From the introductory discussion of the DO balance for open-channel flows, it is apparent that if $K_{2}$ is to be calculated using a DO balance equation, all other parameters appearing in the equation must be known. The procedures for determining these parameters are therefore discussed also.

\section{THEORIES FOR PREDICTING THE REAERATION COEFFICIENT}

The gas-absorption process at the interface between a flowing liquid and a gas has received considerable attention in recent years. The process is very complicated and only superficially understood; so theoretical treatments have been only partially successful. Still, considerable useful information concerning this phenomenon is given in the technical literature of chemical and sanitary engineering. The purpose of this section is to describe the various theories for predicting the reaeration coefficient or the gas-liquid masstransfer coefficient for open-channel flows.

\section{DIFFUSION EQUATION}

A gas moves into and through a flowing liquid by the process of diffusion. This process may be described by the general diffusion equation (Hinze, 1959)

$$
\frac{\partial C}{\partial t}+U_{i} \frac{\partial C}{\partial x_{i}}=\frac{\partial}{\partial x_{i}}\left(D_{m} \frac{\partial C}{\partial x_{i}}-\overline{u_{i} c}\right)+F_{c}
$$

given here in tensor notation. The capital letters represent time-averaged quantities, the lowercase letters represent fluctuating components or the instantaneous deviations from the time-averaged quantities, $C$ is the concentration of the diffusing substance, $U_{i}$ is the velocity in the $x_{i}$ direction, $D_{m}$ is the molecular diffusivity of the dissolved gas in the liquid, $F_{c}$ is a source-sink term, and the overbar on $\overline{u_{i} c}$ indicates an averaging procedure with respect to time. Equation 22 applies to a dispersant which is in solution in a steady-state flow of an incompressible liquid.

With the common assumptions that (1) turbulent diffusion may be described by a turbulent-diffusion coefficient, $\epsilon_{T_{i j}}$, such that

$$
\frac{\partial C}{\partial x_{j}} \epsilon_{T_{i j}}=-\overline{u_{i} c}
$$

(2) the total diffusion may be described by a diffusion tensor, $\epsilon_{i j}$, where the turbulent and molecular diffusion are independent and therefore additive, or

$$
\epsilon_{i j}=\epsilon_{T_{i j}}+D_{m}
$$

and (3) the coordinate axes coincide with the principal axes of the diffusion tensor, Sayre and Chang (1968) reduced equation 22 to

$$
\frac{\partial C}{\partial t}+U_{i} \frac{\partial C}{\partial x_{i}}=\frac{\partial}{\partial x_{i}}\left(\epsilon_{i} \frac{\partial C}{\partial x_{i}}\right)+F_{c} .
$$

For uniform two-dimensional flow in a straight channel of constant cross section, equation 25 may be expressed as

$$
\frac{\partial C}{\partial t}+U \frac{\partial C}{\partial x}=\epsilon_{x} \frac{\partial^{2} C}{\partial x^{2}}+\frac{\partial}{\partial y}\left(\epsilon_{y} \frac{\partial C}{\partial y}\right)+F_{c} .
$$

Equation 26 is expressed in the coordinate system usually used for open-channel flow situations. That is, the mean flow is in the $x$, or longitudinal, direction, and $U$ is the flow velocity in that direction; $y$ is in the vertical direction, or the direction of the channel depth; and the mean flow in the $y$ direction is zero.

Defining averages of the type $\bar{C}=\frac{1}{H} \int_{0}^{H} C d y$, and noting that the mass transport across the lower flow boundary is zero $\left(\left.\epsilon_{y} \frac{\partial C}{\partial y}\right|_{y=0}=0\right)$, one can average equation 26 over the flow depth $H$ and obtain

$$
\frac{\partial \bar{C}}{\partial t}+\frac{\partial}{\partial x}(\overline{U C})=\frac{\partial^{2}}{\partial x^{2}}\left(\overline{\epsilon_{x} C}\right)+\left.\frac{1}{H}\left(\epsilon_{y} \frac{\partial C}{\partial y}\right)\right|_{y=H}+\bar{F}_{c}
$$


Over much of the flow depth of a turbulent openchannel flow, mass transport in the $y$ direction as a result of vertical diffusion is very large compared with the mass transport through the surface. Hence, the vertical gradient of $C$ generally is very small except near the surface and within the bulk of the flow the value of $C$ should approach $\bar{C}$. This has been verified by W. W. Sayre (written commun. 1968), who numerically solved the equation $\frac{\partial C}{\partial t}=\frac{\partial}{\partial y}\left(\epsilon_{y} \frac{\partial C}{\partial y}\right)$. He assumed $\epsilon_{y}$ to be equal to the momentum transport coefficient of a flow having a logarithmic velocity distribution and solved the equation for $C$ as a function of time at 50 points in the vertical using values of $\left.\left(\epsilon_{y} \frac{\partial C}{\partial y}\right)\right|_{y=H}$ corresponding to the range of such values observed for streams in nature. Initially, the flow was assumed to be unsaturated except for the top increment, and he found that even for very short times, there was no appreciable concentration gradient in the vertical. In support of Sayre's work, Churchill, Elmore, and Buckingham (1962) found no vertical concentration gradients in a series of DO measurements in a deep, low-velocity stream, and Edwards and Owens (1962) found that the DO concentration of a slow-flowing English stream was greater than 98 percent of the surface concentration except in a relatively small area near the bed. Also, Whipple, Hunter, Davidson, Dittman, and Yu (1969, p. 116) concluded that the centerline DO concentration at a depth of 1 foot was a good estimate of the mean cross-sectional concentration of DO for the slowflowing Passaic River in New Jersey. When the concentration of the dissolved substance is constant in the vertical, equation 27 may be written

$$
\frac{\partial \bar{C}}{\partial t}+\bar{U} \frac{\partial \bar{C}}{\partial x}=\bar{\epsilon}_{x} \frac{\partial^{2} \bar{C}}{\partial x^{2}}+\left.\frac{1}{H}\left(\epsilon_{y} \frac{\partial C}{\partial y}\right)\right|_{y=H}+\bar{F}_{c} .
$$

It is generally assumed that the diffusion equation in this form is applicable to computing the longitudinal distribution of DO in streams.

Because of the experimental and theoretical evidence indicating no concentration gradient of DO in the vertical direction of an open-channel flow, the use of the overbar on concentration in equation 28 to indicate an average with respect to. the depth of flow in unnecessary. Hence, this overbar on concentration will not be used in the remainder of the report. For consistency, the overbar on the velocity will also be dropped, with the understanding, however, that $U$ is the flow velocity averaged over the depth of flow or the mean velocity of flow in the longitudinal direction.
For reasons of convenience and tradition, completely aside from the fact that the turbulenttransport process in open-channel flows is not sufficiently understood to permit evaluation of $\epsilon_{y}$ near a free surface, the second term on the right in equation 28 is generally expressed using one of two mass-transfer coefficients, $K_{L}$ or $K_{2}$. The more general coefficient $K_{L}$, the liquid-film coefficient, is defined as follows:

$$
K_{L}=\left.\frac{1}{\left(C_{s}-C_{L}\right)}\left(\epsilon_{y} \frac{\partial C}{\partial y}\right)\right|_{y=H},
$$

where $C_{L}$ is the DO concentration in the bulk liquid phase. Comparison of equations 9 and 28 shows that the reaeration coefficient, $K_{2}$, can be expressed in terms of the film coefficient as

$$
K_{2}=K_{L} / H \text {. }
$$

(Eq 9 is the same as eq 28, except the source-sink terms have been lumped into the term $\bar{F}_{c}$ in eq 28.) Theories for the prediction of $K_{L}$ or $K_{2}$ can be checked in certain idealized situations by measurements of $C_{L}$, evaluation of the source-sink terms, and substitution into an integrated version of equation 28.

The prediction of mass-transfer coefficients from solute and solvent properties and from the parameters of the flow is a problem which has received much discussion in the fluid-mechanics literature. At the present time (1971) fairly reliable theories are available for the prediction of heatand mass-transfer coefficients for flows near the solid boundaries in straight circular pipes (Hinze, 1959). These fall into two general categories: the eddy-diffusion or gradient-diffusion theories and the renewal theories.

Because of the gas-liquid interface, side-wall effects, and secondary currents, the mechanics of flow in straight open channels with uniform cross sections are considerably more complex than the mechanics of flow in straight circular pipes. Furthermore, mass-transfer coefficients usually are desired not for straight channels with uniform cross sections but for natural channels, which are neither straight nor of uniform cross section. Because of these complexities, reliable theories comparable to the eddy-diffusivity theories for pipe flows have not been developed for the prediction of mass-transfer coefficients from mean flow parameters in open-channel flows. The theories which have arisen are in general less rigorous, in the fluid-mechanics sense, than are the eddy-diffusivity theories. They fall into three general groups: (1) the conceptual theories, based on assumed mathematical models of the masstransfer processes within the flow; (2) the semi- 
empirical theories, qualitatively relating the mass-transfer coefficients to some measurable flow property, the constants in the relation being determined by multiple regression; and (3) empirical theories resulting from dimensional analysis, again with the constants being determined by multiple-regression techniques. The remainder of this section is devoted to a discussion of the models of these types which have appeared in the literature.

\section{CONCEPTUAL MODELS FOR THE GAS-LIQUID MASS-TRANSFER PROCESS}

Conceptual models are derived from equations describing an interpretation of the physical conditions governing mass transfer at a gas-liquid interface. Of these equations, the one generally used is the differential equation describing nonconvective diffusion. The boundary conditions chosen vary from model to model. In some models, the parameters arising from the solution of the differential equation with the given boundary conditions are not clearly related to the mean flow parameters such as mean velocity, depth of flow, or turbulent intensity. In such models, discovering the relation of the former parameters to the latter is a problem requiring considerable thought and experimental verification. The models may be divided into the following general groups: (1) Film models; (2) renewal, including penetration, filmpenetration, and surface-renewal-damped-eddy diffusivity models; (3) kinetic-theory models; (4) large-eddy models; and (5) turbulent-diffusion models. The usefulness of a particular model ultimately must be determined by how well it predicts reaeration coefficients for natural streams.

\section{FILM MODEL}

The earliest model for mass transfer at a gasliquid interface was the two-film model of Lewis and Whitman (1924). They postulated that laminar films of gas and liquid exist at the interface between the two phases. Because mass transport in a direction perpendicular to the mean-flow direction in a laminar film is by molecular diffusion alone, a process much slower than the turbulent diffusion within the phases, the resistance to mass transfer is concentrated in the two films. The interface between the gas and the liquid films seems to offer no resistance to mass transfer (Sherwood and Pigford, 1952). If the gas and the liquid phases outside of the film region are assumed to be completely mixed, then the mass transport, $N$, of oxygen through the films for the oxygen-water system is

$$
N=\kappa_{L}\left(C_{i}-C_{L}\right)=\kappa_{G}\left(p_{G}-p_{i}\right),
$$

where $C_{L}$ is the concentration of oxygen in the water phase, $C_{i}$ is the concentration of oxygen at the interface, $p_{G}$ and $p_{i}$ are, respectively, the partial pressures of oxygen in the gas phase and at the interface, and $\kappa_{L}$ and $\kappa_{G}$ are, respectively, the liquid-film and gas-film coefficients. Equilibrium conditions usually are assumed at the interface, so that $C_{i}$ and $p_{i}$ are related by Henry's Law, or $p_{i}=h C_{i}$, where $h$ is the Henry's Law constant.

The film coefficients are expected to be dependent on the properties of the liquid and the gas and on the flow parameters, but independent of the concentration of the dissolved gas in the liquid (Sherwood and Pigford, 1952).

Concentrations and partial pressures at the interface are difficult to measure, however; so information on the individual film coefficients is difficult to obtain. Thus, equation 31 usually is written in terms of the overall liquid-film and gasfilm coefficients, or

$$
N=K_{L}\left(C_{s}-C_{L}\right)=K_{G}\left(p_{G}-p_{s}\right),
$$

where $p_{s}$ is the partial pressure corresponding to the saturation concentration, $C_{s}$, and $K_{L}$ and $K_{G}$ are, respectively, the overall liquid-film and gasfilm coefficients for mass transfer.

To be of use in engineering calculations, the overall coefficients should be dependent on the properties of the liquid and of the gas and on the flow parameters, but be independent of the concentration of dissolved gas. From equations 31 and 32 and Henry's Law,

$$
\frac{1}{K_{L}}=\frac{1}{\kappa_{L}}+\frac{1}{h \kappa_{G}}
$$

Thus, for any gas-liquid pair that obeys Henry's Law, the overall liquid-film coefficient is independent of the gas concentration in the liquid. For a slightly soluble gas that does not react chemically with the liquid, $h$ is large, so that equation 33 reduces to

$$
K_{L} \cong \kappa_{L}
$$

Equation 34 is equivalent to saying that the gasfilm resistance is negligible with respect to the liquid-film resistance and that $K_{L}$ is independent of the concentration of dissolved gas for a slightly soluble gas that obeys Henry's Law. The oxygenwater system is such a gas-liquid pair.

Because mass transfer in the laminar film is by molecular diffucion, and if the concentration gradient across the film is assumed to be linear, then

$$
N=D_{m} \frac{\left(C_{s}-C_{L}\right)}{L_{f}}
$$


where $L_{f}$ is the thickness of the film. By comparing equations 32 and 35, it follows that

$$
K_{L}=\frac{D_{m}}{L_{f}}
$$

In equation 36 , the solute and solvent properties are accounted for by the molecular diffusivity, $D_{m}$, and the hydrodynamics of the flow are accounted for by the layer thickness, $L_{f}$. For a truly laminar layer to exist on the surface of an open-channel flow, the flow must be very tranquil. Such flows are seldom found in field situations, however, so that equation 36 does not suffice to describe the variation of the reaeration coefficient with flow parameters. The concept of diffusion into laminar patches of infinitesimal area existing for short times on the water surface before being replaced by liquid from the bulk liquid has been used, however, in subsequent models describing the reaeration process.

\section{RENEWAL MODELS}

The renewal models are analogous to those for predicting mass-transfer coefficients for fluid flows near solid boundaries. In the renewal models, diffusion into a surface layer is generally assumed to be purely molecular. The surface layer is sometimes assumed to be of infinite thickness, and sometimes to have a thickness, $L_{f}$, which may or may not be randomly distributed about an average thickness, $\bar{L}_{f}$. Below the layer, the diffusivity in the liquid is assumed to be infinite, the concentration independent of depth. After diffusion into an element of the surface layer has been taking place for some time, the element is assumed to be replaced by liquid from the bulk flow. The frequency of renewal is governed by an assumed probability density function. The mass-transfer coefficients predicted by these models are functions of the molecular diffusivity, an average renewal frequency, and, generally, an average thickness of the surface layer. The renewal models include (1) the penetration model, (2) the filmpenetration model, and (3) the surface-renewaldamped-eddy diffusivity model.

$$
\text { PENETRATION MODEL }
$$

The penetration model was first postulated by Higbie (1935) and later refined by Danckwerts (1951). Both authors visualized the surface of the liquid as being a laminar film, the thickness of which is large with respect to the depth which can be penetrated by molecular diffusion during the life of the film. The film lifetime is the time elapsed between the formation of the film on the surface and its replacement by fresh liquid from the bulk flow. The difference between the Higbie and the Danckwerts theories lies in the replacement process visualized by the authors.

Molecular diffusion in a laminar layer is governed by the differential equation

$$
\frac{\partial C}{\partial t}=D_{m} \frac{\partial^{2} C}{\partial y^{2}}
$$

For an infinitely deep layer the boundary conditions are:

$$
\begin{array}{lll}
C=C_{L}, & t=0, & y>0, \\
C=C_{L}, & t>0, & y \rightarrow \infty, \\
C=C_{s}, & t>0, & y=0,
\end{array}
$$

where $C_{L}$ is the concentration in the bulk-liquid phase, $y$ is the distance from the interface, and $t$ is the time elapsed since the formation of the interface. The solution to equations 37 and 38 is (Carslaw and Jaeger, 1959)

$$
C=C_{L}+\left(C_{s}-C_{L}\right) \operatorname{erfc}\left[\frac{y}{2 \sqrt{t D_{m}}}\right] \text {, }
$$

and the rate of transport of solute through the surface is

$$
-\left.D_{m} \frac{\partial C}{\partial y}\right|_{y=0}=\left(C_{s}-C_{L}\right) \sqrt{\frac{D_{m}}{\pi t}} .
$$

Higbie (1935) visualized the surface elements as all being of the same age and as all being replaced simultaneously on reaching an age $t_{e}$. Alternatively, the ages of the differential elements of the laminar surface film can be visualized as being distributed according to the distribution function $\phi(t)$ such that

$$
\phi(t)= \begin{cases}\frac{1}{t_{e}}, & 0 \leqq t \leqq t_{e}, \\ 0 & t>t_{e} .\end{cases}
$$

The average rate of mass transport, $\bar{N}$, through a unit of surface area for either case is

$$
\bar{N}=\frac{1}{t_{e}} \int_{0}^{t_{e}}\left(C_{s}-C_{L}\right) \sqrt{\frac{D_{m}}{\pi t}} d t=2\left(C_{s}-C_{L}\right) \sqrt{\frac{D_{m}}{\pi t_{e}}} .
$$

Because $\bar{N}=K_{L}\left(C_{s}-C_{L}\right)$, one has

$$
K_{L}=2 \sqrt{\frac{D_{m}}{\pi t_{e}}} .
$$

Danckwerts (1951) assumed that the surface consists of an infinite number of differential elements of area which have their ages distributed according to

$$
\phi(t)=s e^{-s t},
$$

where $s$ is the average rate of surface renewal. The average rate of mass transport through a unit of surface area is then 


$$
\bar{N}=\int_{0}^{\infty}\left(C_{s}-C_{L}\right) \sqrt{\frac{D_{m}}{\pi t}} s e^{-s t} d t=\left(C_{s}-C_{L}\right) \sqrt{D_{m} s}
$$

so that

$$
K_{L}=\sqrt{D_{m} s}
$$

Neither author attempted to relate either $1 / t_{e}$ or $s$ to the mechanics of flow in open channels, but it is clear that each of these variables must represent a parameter related to the average residence time of the large flow eddies at the surface.

\section{FILM-PENETRATION MODEL}

The film-penetration model was developed by Dobbins (1956) and later by Toor and Marchello (1958). Certain modifications have been discussed in the literature (see, for example, Marchello and Toor, 1963, and Harriott, 1962), but essentially, the film-penetration theory consists of assuming molecular diffusion into laminar surface elements of differential area and thickness, $L_{f}$, which exist on top of a turbulent liquid which has uniform solute concentration, $C_{L}$. The ages of the surface elements are assumed to be distributed according to Danckwerts' (1951) distribution function for surface age as given by equation 44 .

The differential equation governing the diffusion process is equation 37 . The boundary conditions for the film-penetration model are

$$
\begin{array}{llc}
C=C_{L}, & t=0, & 0<y \leqq L_{f}, \\
C=C_{s}, & t \geqq 0, & y=0, \\
C=C_{L}, & t \geqq 0, & y=L_{f} .
\end{array}
$$

Dobbins (1956) obtained as the solution to equations 37 and 47

$$
C=C_{L}+\left(C_{s}-C_{L}\right) \sum_{n=0}^{\infty}\left(\operatorname{erf} \frac{n+1-\frac{y}{2 L_{f}}}{\sqrt{\frac{D_{m} t}{L_{f}^{2}}}}-\operatorname{erf} \frac{n+\frac{y}{2 L_{f}}}{\sqrt{\frac{D_{m} t}{L_{f}^{2}}}}\right)
$$

and using the age-distribution function of Danckwerts (1951), he obtained

$$
K_{L}=\sqrt{D_{m} s} \operatorname{coth}\left(\frac{s L_{f}^{2}}{D_{m}}\right)^{1 / 2}
$$

When the film-renewal rate, $s$, becomes very small, equation 49 approaches the film limit, or $K_{L}=D_{m} / L_{f}$, while for $\sqrt{s L_{f}^{2} / D_{m}}>3.0$, equation 49 gives essentially the penetration limit, or $K_{L}=\sqrt{D_{m} s}$.

Marchello and Toor (1963) presented a modification of the film-penetration model in which the surface layer is assumed not to be replaced but to be instantaneously mixed at times which are distributed according to the age-distribution function of Danckwerts (1951). In the periods between mixings, diffusion is governed by equation 37 . They obtained for the mass-transfer coefficient

$$
K_{L}=\frac{\sqrt{D_{m} s}}{2} \cdot \frac{\left[1+\cosh \left(\frac{s L_{f}^{2}}{D_{m}}\right)^{1 / 2}\right]}{\sinh \left(\frac{s L_{f}^{2}}{D_{m}}\right)^{1 / 2}}
$$

For small renewal rates, equation 50 approaches the film-model limit, and for large renewal rates it approaches one-half the penetration-model prediction for $K_{L}$.

Another modification of the film-penetration model was presented by Harriott (1962). His theory was designed for application to mass transport at fluid-solid interfaces, but it could probably be applied also to open-channel flows, especially in the presence of surfactants. In this theory it is postulated that diffusion is molecular, according to equation 37, and that renewal occurs not necessarily all the way to the surface but to some distance $y_{1}$ below the surface. In applying this theory, one must solve equation 37 with the boundary conditions

$$
\begin{array}{lcc}
C=C_{s}, & y=0, & t \geqq 0, \\
C=C_{L}, & y \rightarrow \infty, & t \geqq 0, \\
C=f(y) & 0 \leqq y \leqq \infty, & t=0 .
\end{array}
$$

The probability density functions used by Harriott (1962) to describe the distributions of the renewal period, $t$, and of the distance below the surface to which renewal occurs, $y_{1}$, were of the form

$$
p(f)=\frac{1}{\Gamma(\alpha) \beta^{\alpha}} f^{\alpha-1} \exp (-f \mid \beta)
$$

where $f$ is a dummy variable (signifying $t$ or $y_{1}$ ), $\alpha$ and $\beta$ are distribution parameters, and $\Gamma$ denotes the gamma function. In this model, $K_{L}$ cannot be obtained analytically, so it must be obtained using numerical techniques. Harriott found that at the penetration-model limit, the numerical procedures gave $K_{L} \propto \sqrt{D_{m} S}$ as expected; however, at what would ordinarily be the film-model limit, he found $K_{L} \propto y_{1}^{n-1} D_{m^{1-n / 2}} s^{n / 2}$, where $0 \leqq n \leqq 1$ and $n$ is highly sensitive to the distribution parameters $\alpha$ and $\beta$.

Several methods have been presented for determining the parameters in the Dobbins model in terms of the parameters of the turbulent-flow system. Not much has been done along these lines with the Marchello and Toor model or with the Harriott model; however, the concepts used in the case of the Dobbins model should be adaptable to these models as well.

The earliest method for relating the parameters of the Dobbins model to the parameters of openchannel flow was presented by O'Connor and Dobbins (1958). They pointed out that for rivers, $\operatorname{coth}\left(\frac{s L_{f}^{2}}{D_{m}}\right)^{1 / 2} \cong 1$, so that $K_{L} \cong \sqrt{D_{m} s}$, and all that needs to be determined in terms of the flow param- 
eters is $s$, the mean rate of surface renewal. For determining $s$, river turbulence is divided into two classes: nonisotropic, and essentially isotropic at the water surface. For both types of turbulence, $s$ is defined as the ratio of the vertical turbulent intensity to the mixing length, at the water surface. For a Chezy $C$ less than 17, O'Connor and Dobbins assumed nonisotropic turbulence to exist, and using the definition of the Prandtl mixing length and the von Karman logarithmic velocity law, they obtained

$$
s=\frac{\left(D_{m} g S\right)^{1 / 2}}{\kappa H},
$$

where $H$ is the flow depth, $S$ is the channel slope, $g$ is the gravitational constant, and $\kappa$ is the slope parameter in von Karman's logarithmic velocity law. Substitution of equation 53 into equation 49 leads to

$$
k_{L}=480 \frac{D_{m}^{1 / 2} S^{1 / 4}}{H^{1 / 4}} \text {, or } k_{2}=\frac{480 D_{m^{1 / 2}} S^{1 / 4}}{H^{5 / 4}},
$$

where $k_{L}$ is in feet per day, $H$ is in feet, and $D_{m}$ is in square feet per day. For a Chezy $C$ greater than 17 , isotropic turbulence is assumed to exist. For this case, O'Connor and Dobbins (1958) used measurements made by Kalinske (1943) on the Mississippi River between Dubuque, Ia., and Cairo, Ill., which showed that the vertical turbulent intensity was approximately $0.1 U$ and that the mixing length was approximately $0.1 H$; hence,

$$
s=\frac{0.1 U}{0.1 H}=\frac{U}{H} \text {. }
$$

Thus

$$
k_{L}=127\left(\frac{D_{m} U}{H}\right)^{1 / 2} \text {, or } k_{2}=\frac{127\left(D_{m} U\right)^{1 / 2}}{H^{3 / 2}},
$$

where the mean flow velocity $U$ is in feet per second and all other units are the same as in equation 54. In equations 55 and $56, k_{L}$ and $k_{2}$ are for use with the base 10 .

Another theory relating the parameters of the Dobbins model to the mean flow parameters was presented by Dobbins (1946b). He postulated that the effective film thickness must be related to the smallest surface eddies into which the gas can penetrate by molecular diffusion before being carried into the flow below. Using Kolmogoroff's limit for the size of the energy-dissipating eddies, he obtained

$$
L_{f}=c_{3}\left(\frac{\nu^{3}}{E_{\xi}}\right)^{1 / 4}=c_{4}\left(\frac{\nu^{3}}{E}\right)^{1 / 4},
$$

where $c_{3}$ and $c_{4}$ are constants, $\nu$ is the kinematic viscosity of the liquid, and $E_{\xi}$ and $E$ are, respectively, the energy dissipated per unit mass of liquid at the surface, and the energy dissipated per unit mass of liquid by the entire flow. Dobbins also postulated that the rate of expenditure of energy required to overcome the surface tension and replace the surface liquid is proportional to the turbulent energy per unit mass of liquid near the surface. Thus

$$
s=\frac{c_{1} \rho L_{f} E_{\xi}}{\sigma}=\frac{c_{5}}{c_{4}{ }^{3}} \frac{p v^{3 / 4} E^{3 / 4}}{\sigma},
$$

where $c_{1}$ and $c_{5}$ are constants, $\rho$ the mass density of the liquid, and $\sigma$ its surface tension. It can further be shown that

$$
s L_{f}^{3}=c_{5} \frac{\rho \nu^{3}}{\sigma} \text {. }
$$

In equations 57 and $58, c_{5}$ is dependent only on solute and solvent properties, whereas $c_{4}$ is dependent on the dynamics of the flow system. Dobbins reported $c_{5}$ to be 14.3 for the oxygen-water system at $20^{\circ} \mathrm{C}$. He obtained

$$
c_{4}=0.65+15,000\left[\frac{\left(\frac{v^{3}}{E}\right)^{1 / 4}}{H}\right]^{2}
$$

from a plot of experimental results that contains considerable scatter. In the closure to his $1964 \mathrm{~b}$ paper, Dobbins (1965) presented a prediction equation derived from the same data, but which is more convenient to use than equations 60,59 , and 57 in combination with equation 49 . The prediction equation for $k_{L}$ is

$$
k_{L}=\frac{0.12 B_{\mathrm{F}} A E^{3 / 8} \operatorname{coth}\left(\frac{B E^{1 / 8}}{c_{4}{ }^{1 / 2}}\right)}{c_{4}{ }^{3 / 2}},
$$

where

$$
\begin{aligned}
B_{\mathrm{F}} & =1.0+\mathrm{F}^{2} \\
c_{4} & =0.9+\mathbf{F} \\
A & =9.68+0.054\left(T-20^{\circ}\right) \\
B & =0.976+0.0137\left(30^{\circ}-T\right)^{3 / 2} \\
E & =30.0 S U \\
\mathbf{F} & =U / \sqrt{g H}
\end{aligned}
$$

and where the slope, $S$, is in feet per thousand feet; $T$ is the temperature, in degrees Celsius; and $k_{L}$ is in feet per day.

In a modification of Dobbins' (1964b) theory, Metzger and Dobbins (1967) used a surface compressional modulus, $M_{s}$, in place of the surface tension, $\sigma$. Following arguments similar to those used by Dobbins (1964b), they obtained

$$
\begin{gathered}
s=\frac{c_{1}{ }^{\prime} c_{2}^{3 / 4}\left(c_{3}{ }^{\prime}\right)^{3} \rho \nu^{3 / 4} E^{3 / 4}}{M_{s}}, \\
L_{f}=c_{4}\left(\frac{\nu^{3}}{E}\right)^{1 / 4},
\end{gathered}
$$

and

$$
s L_{f}^{3}=c_{5}^{\prime} \frac{\rho \nu^{3}}{M_{s}},
$$

where $c_{2}$ and $c_{4}$ are the same constants as in the Dobbins theory and $c_{1}^{\prime}, c_{3}^{\prime}$, and $c_{5}^{\prime}$ are new constants, and where $c_{5}$ is independent of the flow dynamics. For distilled water, Metzger and Dobbins (1967) 
found $\frac{c_{5}^{\prime}}{M_{s}}=1.3 \frac{\mathrm{sec}^{2}}{\text { gram }^{2}}$, and Metzger (1968) found this same value in the presence of $0.5 \mathrm{mg} / \mathrm{l}$ of alkyl benzene sulfonate (a surfactant) in water. The significance of having $M_{s}$ rather than $\sigma$ in the denominator of equation 65 is that $M_{s}$ increases in the presence of surface-active agents while $\sigma$ decreases. Thus equation 65 is in qualitative agreement with the observation that surface-active agents retard surface renewals.

The localized-mixing model of Marchello and Toor (1963) can be applied not only to the surface layer of an open-channel flow but also to lower layers within this type of flow and to all layers in other types of flow, such as pipe flows. When this is done, for any layer:

$$
\frac{\epsilon_{y}}{D_{m}}=\sqrt{\frac{\beta^{\prime}}{D_{m}}} \cdot \frac{1+\cosh 2 \sqrt{\frac{\beta^{\prime}}{D_{m}}}}{\sinh 2 \sqrt{\frac{\beta^{\prime}}{D_{m}}}}-1,
$$

where $\epsilon_{y}$ is the eddy diffusivity for mass, and $\beta^{\prime}$ is the turbulence parameter for the layer, such that

$$
\beta^{\prime}=s\left(\frac{L_{f}}{2}\right)^{2} \text {. }
$$

Similarly, after applying the localized-mixing model to momentum transfer, Marchello and Toor obtained

$$
\frac{\epsilon_{v}}{v}=\sqrt{\frac{\beta^{\prime}}{v}} \frac{1+\cosh 2 \sqrt{\frac{\beta^{\prime}}{v}}}{\sinh 2 \sqrt{\frac{\beta^{\prime}}{v}}}-1,
$$

where $\epsilon_{\nu}$ is the eddy diffusivity for momentum. Thus, if one can determine the momentum eddy diffusivity for a particular layer, one can solve equation 68 for $\beta^{\prime}$ and determine the eddy diffusivity for mass for the layer from equation 66 .

The combination of the film concept of Lewis and Whitman (1924) and the penetration concept of Higbie (1935) and Danckwerts (1951) into one model has considerable merit in that the combined model can accurately predict reaeration coefficients for natural streams over a much wider range of conditions than can either of the models alone. The film-penetration models, however, are idealized in that the diffusion process is visualized as taking place in two separate steps, rather than as being governed by the classical diffusion equation embodying both molecular and turbulent diffusion. The first step is molecular diffusion; the second step is turbulent diffusion, or nongradient diffusion, embodied by a renewal or mixing process. This idealization will, of course, lead to difficulties in relating model parameters to flow parameters, and lead to deviations of the predicted from the true mass-transfer coefficients in situations where the flow conditions differ appreciably from those under which the experimental model coefficients were determined. For the Dobbins (1956) model, the O'Connor and Dobbins (1958) method for determining $s$ is perhaps overly simplified, and it requires no experimental coefficients to be evaluated. That this method predicts $k_{L}$ values for natural streams as well as it does is therefore a little surprising. The Dobbins (1964b) and Metzger and Dobbins (1967) methods are more rational and more sophisticated, and they require the evaluation of two experimental coefficients. Equation 60 used by Dobbins (1964b) to predict $c_{4}$ was obtained from a plot of field data that contains considerable scatter. This scatter is, however, apparently of minor importance, because if one checks the prediction equation using the data from which equation 60 was evaluated, there is only a small amount of scatter about the line of perfect agreement between predicted and observed $k_{2}$ values.

\section{SURFACE-RENEWAL-DAMPED-EDDY DIFFUSIVITY MODEL}

The surface-renewal-damped-eddy diffusivity model was presented by King (1966), who postulated that the mass transport near the surface is due not only to $D_{m}$, but also to turbulent diffusion caused by small-scale eddies. The coefficient characterizing the turbulent diffusion caused by the small-scale eddies is the eddy diffusivity for mass, $\epsilon_{y}$, which is assumed to be of the form $\epsilon_{y}=a y^{n}$, where $a$ and $n$ are parameters independent of time, and $y$ is the distance from the interface. The surface renewals are assumed to be due to the large-scale eddies, and the surface ages are assumed to be distributed according to the distribution function for uniform surface ages (eq 41).

The diffusion equation applicable to the above model is

$$
\frac{\partial C}{\partial t}=\frac{\partial}{\partial y}\left[\left(D_{m}+a y^{n}\right) \frac{\partial C}{\partial y}\right]
$$

and the boundary conditions used by King (1966) are

$$
\begin{array}{lll}
C=C_{L}, & t=0, & y>0, \\
C=C_{L}, & t>0, & y \rightarrow \infty, \\
C=C_{s}, & t>0, & y=0 .
\end{array}
$$

Equation 69 along with the boundary conditions 70 can be solved analytically only in special cases, such as for $a=0$ and all $n$; for all $a$ and $n=0,1, \infty$; and for all $a$ and all $n$ with large $t$. For large $t$, the solution of equations 69 and 70 (King, 1966) gives

$$
\frac{K_{L}}{a^{1 / n} D_{m}^{1-1 / n}}=\frac{n}{\pi} \sin \frac{\pi}{n}
$$


where $t$ may be interpreted as the surface age at renewal.

If the surface-renewal-damped-eddy diffusivity model is to be useful in predicting film coefficients, the parameters $a$ and $n$ in equation 69 as well as an average surface age, $\bar{t}$, must be estimated. From equation 71 it is seen for large surface ages that the exponent $n$ can be estimated from the variation of $K_{L}$ with molecular diffusivity, if the other factors remain constant. Kozinski and King (1966) concluded that $K_{L} \propto D_{m}{ }^{0.75}$ for gas-liquid transfer at large surface ages; thus $n=4$. From the work of Calderbank and Moo-Young (1961), King (1966) concluded that

$$
a=0.006 \frac{E_{V} \rho}{\mu^{2}},
$$

where $E_{V}$ is the energy-dissipation rate per unit of liquid volume, $\rho$ is the mass density of the liquid, and $\mu$ is the absolute viscosity of the liquid. From figure 3 of King (1966) and from equation 71 for $n=4$, it is seen that if surface-renewal effects control, the surface-renewal-damped-eddy diffusivity model yields film-coefficient predictions of the same order as those measured in large open channels and rivers.

The surface-renewal-damped-eddy diffusivity model is physically more realistic than previous models because the artifice of a surface layer is avoided. Also, the eddy diffusivity is specifically accounted for in the diffusion equation, rather than by being lumped into surface renewal, so that gradient and nongradient diffusion are more clearly separated. Practically, however, the model is cumbersome in that three parameters must be evaluated, and equation 69 must be solved numerically in most cases. King (1966) showed that if $n=4$ and $a$ is given by equation $72, s$ should be proportional to $\frac{\rho}{\mu}\left[\frac{E_{V} H}{\rho}\right]^{2 / 3}$ for open-channel flows. This has not been checked experimentally.

\section{KINETIC THEORY}

The kinetic theory as developed by Miyamoto (1932a, b) was presented by Krenkel and Orlob (1963). The model is based on the frequencies and velocities of bombardment of the interface by solute molecules from both the liquid and the gas phases. The resulting equation is

$$
k_{L} \propto \frac{1}{1,000}\left(\frac{R_{G} T^{\prime}}{2 \pi M}\right)^{1 / 2} \exp \left[-\left(M / 2 R_{G} T^{\prime}\right)\left(\mu_{0}^{\prime}\right)^{2}\right],
$$

where $R_{G}$ is the ideal-gas constant, $T^{\prime}$ is the absolute temperature, $M$ is the molecular weight of the gas, and $\mu_{0}^{\prime}$ is the velocity which a solute molecule must attain normal to the interface to be able to leave the liquid.

If equation 73 is to account for the turbulence properties of the flow as well as for solute and solvent properties, the critical parameter would have to be $\mu_{0}^{\prime}$. That $\mu_{0}^{\prime}$ could account for the turbulence properties of the flow is reasonable, because $M\left(\mu_{0}^{\prime}\right)^{2} / 2$ may be viewed as an activation energy term, and as pointed out by Bird, Stewart, and Lightfoot $(1960$, p. 27$)$, the activation energy of liquids is influenced by local values of the shear stress, which can in turn be related to local turbulence properties. Apparently, no studies, either theoretical or experimental, have been made in which such relations have been presented.

Another kinetic-theory model was presented by Tsivoglou (1967). It too arises from consideration of the difference between the rate of entry and the rate of loss of gas (oxygen) molecules from a surface layer of liquid (water). The loss rate, $r_{0}$, is

where

$$
r_{0}=b\left(L_{f} C n_{s} A_{s}\right)
$$

$C=$ concentration of gas in the liquid,

$L_{f}=$ thickness of surface layer from which gas molecules can escape to the atmosphere,

$n_{s}=$ number of new surface layers exposed per unit of time,

$A_{s}=$ surface area, and

$b=$ the proportion of available molecules in the surface layer that actually escape.

The entry rate, $r_{e}$, which is dependent on the gas concentration in the air above the water, is a constant. It is equal to the loss rate at $C=C_{s}$, so that

$$
r_{e}=b\left(L_{f} C_{s} n_{s} A_{s}\right)
$$

The time rate of change of the liquid concentration is then

$$
\frac{d C}{d t}=\frac{r_{e}-r_{0}}{V}=\frac{b L_{f} n_{s} A_{s}}{V}\left(C_{s}-C\right),
$$

so that

$$
K_{2}=\frac{b L_{f} n_{s} A_{s}}{V} .
$$

Because $\frac{A_{s}}{V}=\frac{1}{H}$, in which $V$ is the volume of the system and $H$ is its depth,

$$
K_{L}=b L_{f} n_{s} .
$$

Equation 78 is mostly of theoretical interest, because at the present time none of the parameters on the right-hand side can be measured. However, some of the results obtained by Tsivoglou (1967) by manipulation of the equations of this model are of interest. First, because the entry rate is a function only of the gas concentration in the layer above the fluid, Tsivoglou concluded that the 
factor $b$ varies with temperature according to the relation

$$
(b)_{T_{2}}=(b)_{T_{1}} \frac{\left(C_{s}\right)_{T_{1}}}{\left(C_{s}\right)_{T_{2}}}
$$

where the subscripts indicate two different temperatures. Combining equation 79 with equation 77 yields

$$
\frac{K_{2_{T_{2}}}}{K_{2_{T_{1}}}}=\frac{C_{s_{T_{1}}}}{C_{s_{T_{2}}}}=\theta^{\left(T_{2}-T_{1}\right)},
$$

in which $\theta$ is a temperature-correction coefficient for the mass-transfer coefficient. The temperature-correction coefficient will be discussed in greater detail later. At present, the value usually accepted for $\theta$ is 1.0241 , given by Elmore and West (1961). The values calculated by Tsivoglou for $\theta$ from equation 80 for oxygen and water for temperatures from $0^{\circ}$ to $30^{\circ} \mathrm{C}$ range from 1.018 to 1.026, which lends credence to Tsivoglou's model.

Tsivoglou (1967) presented further support for his model. Assuming the average molecular transport within the body of a turbulent liquid to be due to an average concentration gradient between volume elements, and equating the mass transfer at the surface to the average transfer between the volume elements, Tsivoglou showed that for the simultaneous transfer of two gases,

$$
\frac{\left(K_{2}\right)_{1}}{\left(K_{2}\right)_{2}}=\frac{\left(D_{m}\right)_{1}}{\left(D_{m}\right)_{2}}
$$

When considering the ratio of the mass-transfer coefficients of two gases, equation 81 leads to the same conclusion as the film-model equation (36)namely that the ratio of the mass-transfer coefficients of the two gases is directly proportional to the first power of the ratio of their molecular diffusivities.

In further developing his model, Tsivoglou (1967) pointed out that if mass transport through the surface is governed by Einstein's law of diffusion, then

$$
\frac{\left(D_{m}\right)_{1}}{\left(D_{m}\right)_{2}}=\frac{d_{2}}{d_{1}}
$$

in which $d_{1}$ and $d_{2}$ are the molecular diameters of the two gases. From equations 82 and 81 ,

$$
\frac{\left(K_{2}\right)_{1}}{\left(K_{2}\right)_{2}}=\frac{d_{2}}{d_{1}}
$$

Tsivoglou, O'Connell, Walter, Godsil, and Logsdon (1965) and Tsivoglou (1967) completed an extensive series of measurements of the simultaneous transfer of several pairs of gases across a water interface. Two types of apparatus were used: one in which both gases were transferred simultaneously into the water, and one in which a second gas was transferred out of the water as oxygen was transferred into it. A range of temperature and turbu- lence conditions was studied for each pair of gases and each type of apparatus. On the basis of these measurements, Tsivoglou (1967) concluded that the ratio of the gas-transfer coefficients was a constant within the limits of experimental error for the range of temperature and turbulence conditions studied. This supports his model as given in equation 77.

Tsivoglou (1967) suggested that his experimental measurements of the gas-transfer ratios for the different pairs of gases together with moleculardiameter data from the literature support equation 83. However, a linear regression analysis of the logarithms of the transfer-coefficient ratios and of the molecular-diameter ratios yielded

$$
\frac{\left(K_{2}\right)_{1}}{\left(K_{2}\right)_{2}}=1.06\left(\frac{d_{2}}{d_{1}}\right)^{1.26}
$$

where the 95-percent confidence limits on the exponent are 1.09 and 1.44 . Other experimental evidence suggests that equation 83 and the argument that $D_{m}$ can be obtained from Einstein's law of diffusion are not strictly valid. Kozinski and King (1966) found that the exponent on $D_{m}$ in the relation $k_{L} \propto D_{m}{ }^{n}$ varied from about 0.50 to 0.75 for the stirred-tank data of Dobbins (1964a); these values are considerably different from the $\mathbf{1 . 2 6}$ indicated by equations 84 and 82 .

These apparent discrepancies suggest that Einstein's law of diffusion is not strictly applicable to this situation, but note that these discrepancies do not in any way reflect on the validity of the model as represented by equation 81 . The only requirement of the model here is that the gastransfer-coefficient ratio be a constant, as was found in a laboratory study by Tsivoglou, O'Connell, Walter, Godsil, and Logsdon (1965) and Tsivoglou (1967).

\section{LARGE-EDDY MODEL}

The large-eddy model as presented by Fortescue and Pearson (1967) postulates that the masstransfer properties of a turbulent surface can be modeled by a series of steady square roll cells. The cells touch the surface of the flow and are convected downstream with a velocity equal to the mean flow velocity at the surface. The roll cells are taken to be $\Lambda$ on a side, where $\Lambda$ is the turbulent macroscale of the flow. The velocity pattern within a cell is given by

$$
\begin{gathered}
u_{e}=A_{e} \sin \frac{\pi x}{\Lambda} \cos \frac{\pi y}{\Lambda} \\
v_{e}=-A_{e} \cos \frac{\pi x}{\Lambda} \sin \frac{\pi y}{\Lambda} .
\end{gathered}
$$


The parameter $A_{e}$ is a measure of $\bar{E}$, the average kinetic energy of the roll cell, because

$$
\bar{E}=\frac{1}{2 \Lambda^{2}} \int_{0}^{\Lambda} \int_{0}^{\Lambda}\left(u_{e}^{2}+v_{e}^{2}\right) d x d y=\frac{1}{4} A_{e}^{2} .
$$

The differential equation describing the gas concentration distribution in the large-eddy model is

$$
u_{e} \frac{\partial C}{\partial x}+v_{e} \frac{\partial C}{\partial y}=D_{m}\left(\frac{\partial^{2} C}{\partial x^{2}}+\frac{\partial^{2} C}{\partial y^{2}}\right),
$$

with boundary conditions

$$
\begin{array}{lcc}
C=C_{s}, & y=0, & 0 \leqq x \leqq \Lambda, \\
\frac{\partial C}{\partial x}=0, & 0 \leqq y \leqq \Lambda, & x=n \Lambda, \\
C=C_{L}, & y=\Lambda, & 0 \leqq x \leqq \Lambda,
\end{array}
$$

where $n$ is an integer. Equation 87 with boundary conditions 88 must be solved numerically.

Dimensional analysis of equation 87 along with equation 85 shows that the characteristic parameter describing the large-eddy model is $P$, the Peclet number, where $\mathrm{P}=\Lambda A_{e} / D_{m}$. Fortescue and Pearson (1967) reported that for $P$ values "over the range that was revelant," the numerical solution of equations 85,87 , and 88 yields

$$
k_{L}=1.46\left(\frac{D_{m} \bar{u}^{\prime}}{\Lambda}\right)^{1 / 2}
$$

where $\overline{u^{\prime}}$ is the longitudinal turbulent intensity. Using the O'Connor and Dobbins (1958) relation for $\bar{u}^{\prime} / \Lambda=U / H$, Fortescue and Pearson reported that their model gives "rather better" predictions than the Higbie (1935) penetration model (eq 43) with $1 / t_{e}=\overline{u^{\prime}} \mid \Lambda=U / H$, for the river data used by O'Connor and Dobbins (1958).

The large-eddy model is different in concept from the other models presented in this section, but the concept is as realistic as, for example, the film-penetration concept. An individual cell is a considerably idealized turbulent eddy, and the surface film is a film of eddies, rather than a stagnant film. The model avoids the question of surface age by allowing the age to vary in a set pattern across the top of the cell. It would be interesting to find the effect on the computed film coefficient of assumed distribution functions for varying cell sizes and kinetic energy. A more realistic relation for surface values of $\overline{u^{\prime}} / \Lambda$ might improve the predictions made by the model.

\section{TURBULENT-DIFFUSION MODEL}

In discussing the turbulent-diffusion model, Kishinevsky (1955) postulated for high turbulence levels that the turbulent diffusivity, $\epsilon_{y}$, is not damped out at the water surface. He further stated that the turbulent diffusivity at the surface is much larger than the molecular diffusivity, so that

$$
k_{L}=\frac{2}{\sqrt{\pi}} \sqrt{\epsilon_{y} s} .
$$

An example in which equation 90 can be said to govern was presented by Kishinevsky and Serebryansky (1956). They reported studies in a rapidly stirred tank in which the measured film coefficients for hydrogen, oxygen, and nitrogen were all roughly the same, despite the fact that the molecular diffusivity of hydrogen is about twice that of oxygen and nitrogen. They also reported, however, that at lower stirring speeds the molecular diffusivity influenced the measured film coefficient. The fact that in open-channel flows the measured values of $k_{L}$ are a function of temperature indicates that in this type of flow turbulence levels do not reach a magnitude sufficient to make equation 90 applicable.

Note that the models discussed predict a range of dependence of $k_{L}$ on $D_{m}$ varying from an exponent of 1 for the film model to 0 for the turbulentdiffusion model. The film-penetration model predicts an exponent on $D_{m}$ which varies from 0.5 to 1.0 , whereas the surface-renewal-damped-eddy diffusivity model can predict an exponent anywhere from 0 to 1.0. Experimental data taken in stirred tanks (Kozinski and King, 1966) indicated that the exponent is in the 0.5 to 0.75 range. Therefore, each model (with the possible exception of the film model) seemingly is applicable to a limited range of turbulence conditions. However, the surface-renewal-damped-eddy diffusivity model is the only one which is applicable over the entire range of turbulence conditions.

All the conceptual models for the gas-liquid mass-transfer process involve two physical properties of the oxygen-water system. These are the molecular diffusivity and the saturation concentration for oxygen in water. Each of these properties has been studied in considerable detail, and the following paragraphs summarize briefly the results of these studies.

The determination of the molecular diffusivities of dissolved gases in liquids was reviewed by Himmelblau (1964), and the experimental determinations of the molecular diffusivity of oxygen in water were discussed by Duda and Vrentas (1968).

The experimental data for molecular diffusivity at $25^{\circ} \mathrm{C}$ fall into two groups, one group indicating a value of about $2.5 \times 10^{-5} \mathrm{~cm}^{2}$ per sec (square centimeters per second) and the second group indicating a value of about $2.0 \times 10^{-5} \mathrm{~cm}^{2}$ per sec. The larger values in general have been used (Isaacs 
and Gaudy, 1968; Metzger and Dobbins, 1967; Kozinski and King, 1966; Dobbins, 1964b; O'Connor and Dobbins, 1958). Himmelblau (1964) also believed that the smaller values at $25^{\circ} \mathrm{C}$ were probably incorrect, and Baird and Davidson (1962) believed that the larger values obtained with the wetted sphere procedure were more accurate than the values obtained with the annular jet. On the other hand, Kolthoff and Izutsu (1964) concluded that a modified equation incorporating a second perturbation term should be used in the calculation of oxygen diffusivities from polarographic measurements. This modification resulted in diffusivities of $1.94 \times 10^{-5}$ and $1.97 \times 10^{-5} \mathrm{~cm}^{2}$ per sec at $25^{\circ} \mathrm{C}$ as compared with values of $2.56 \times 10^{-5}$ and $2.50 \times 10^{-5}$ $\mathbf{c m}^{2}$ per sec obtained without the second perturbation term. Duda and Vrentas (1968) concluded from their consideration of the available experimental data that the evidence tended to support the smaller diffusivity values, in agreement with their laminar-jet result. Controversy still exists concerning the correct value for the molecular diffusivity of oxygen in water; so diffusivity data for the oxygen-water system should be used with this consideration in mind.

The saturation concentrations presented by the American Public Health Association (1960, 1965) and the Committee on Sanitary Engineering Research (1960) in general have been accepted and used as standards. Truesdale, Downing, and Lowden (1955) obtained values that were significantly different from the American Public Health Association values, but later work by Montgomery, Thom, and Cockburn (1964) showed that the values were low because of losses of iodine vapor during analysis for DO. More recent studies of the saturation concentration were discussed by Montgomery (1969). He concluded that the values from these studies were in general slightly smaller than the values reported by the American Public Health Association $(1960,1965)$ and, for temperatures above $15^{\circ} \mathrm{C}$, were slightly larger than values obtained by the Committee on Sanitary Engineering Research (1960).

The saturation concentration decreases with increasing temperature and decreases with decreasing barometric pressure. The temperature dependence of the saturation concentrations obtained by the Committee on Sanitary Engineering Research (1960) was expressed in terms of a cubic equation. The dependence on barometric pressure, $p_{B}$, may be expressed in terms of a simple ratio, or

$$
C_{s_{2}}=C_{s_{1}} \frac{p_{B_{2}}}{p_{B_{1}}} .
$$

For elevations above 3,000 feet and temperatures above $25^{\circ} \mathrm{C}$, corrections for the water vapor pressure should be made (Am. Public Health Assoc., 1965 , p. 409).

Equation 91 demonstrates a fact perhaps not generally recognized. For a temperature of $20^{\circ} \mathrm{C}$ and a pressure of $760 \mathrm{~mm} \mathrm{Hg}$ (millimeters of mercury), the saturation concentration is 9.02 $\mathrm{mg} / \mathrm{l}$ (Committee on Sanitary Engineering Research, 1960). If a concentration of $5.0 \mathrm{mg} / \mathrm{l}$ is required for prevention of fish kills, then a range of about $4 \mathrm{mg} / \mathrm{l}$ of DO is available for a stream at an elevation of about sea level-for example, along the east coast of the United States. However, for a stream at an elevation of about 5,000 feet in the Rocky Mountain region, the saturation concentration is about $7.5 \mathrm{mg} / \mathrm{l}$; hence, a range of only about $2.5 \mathrm{mg} / \mathrm{l}$ of $\mathrm{DO}$ is available for use in oxidation of wastes.

The saturation concentration decreases with increasing chloride concentration in the water. The effect ranges from a decrease of $0.017 \mathrm{mg} / \mathrm{l}$ of $\mathrm{DO}$ per $100 \mathrm{mg} / \mathrm{l}$ of chloride at $0^{\circ} \mathrm{C}$ to a decrease of $0.008 \mathrm{mg} / \mathrm{l}$ of $\mathrm{DO}$ per $100 \mathrm{mg} / \mathrm{l}$ of chloride at $30^{\circ} \mathrm{C}$ (Am. Public Health Assoc., 1965, p. 409). Sewage in the water may affect the DO saturation value. Moore (1938) found that the solubility of oxygen in domestic sewage was about 95 percent of the solubility in distilled water, and Rand (1959) concluded that the solubility of oxygen in sewage was not significantly different from the solubility in pure water.

\section{SEMIEMPIRICAL MODELS}

The semiempirical models result from a consideration and combination of the factors that should affect mass transfer at a free surface. These models rely on regression-correlation analysis of experimental data for establishment of a prediction equation and for verification of the assumed relations between the mass-transfer coefficient and the flow parameters. In general, the steps in the derivation of these models are: (1) From intuition, a knowledge of previous conceptual models, a knowledge of the results of previous empirical studies, or a combination of these, to postulate that the film coefficient $k_{L}$ (or the reaeration coefficient, $k_{2}$ ) is a function of certain of the parameters of the flow; (2) if necessary, to manipulate the assumed relation to obtain a relation for $k_{L}$ (or $k_{2}$ ) in terms of easily measured mean-flow parameters; and (3) to use regression-correlation analyses to fit experimental data to the assumed relation and to 
check its validity. The determination of the usefulness of these models must be based on the reliability of the data from which they were derived, on how well they predict mass-transfer coefficients for natural streams, and on ease of use. The first two points are discussed in a later section of this paper; the last, as the models are presented. The models discussed in this section are those presented by Krenkel (1960) and by Thackston (1966). The limits on the ranges of data from which the semiempirical and empirical models were derived are discussed in a later section.

KRENKEL MODEL

The Krenkel model was presented by Krenkel (1960) and Krenkel and Orlob (1963), who reasoned that

$$
k_{2} \propto\left[\frac{D_{i}}{\nu}\right]\left[\frac{\epsilon}{H^{2}}\right],
$$

where the first term in brackets is characteristic of the rate of absorption at the surface, and the second term is characteristic of the rate of surface renewal. In equation $92, D_{i}$ is a combination of molecular and turbulent diffusivity at the surface, and $\epsilon$ is an eddy-diffusion coefficient. However, both Krenkel (1960) and Krenkel and Orlob (1963) believed that the reaeration coefficient is better represented by

$$
k_{2} \propto s \exp \left[-E_{a} / R_{G} T^{\prime}\right],
$$

where $s=\epsilon / H^{2}, E_{a}$ is the activation energy for oxygen absorption, $R_{G}$ is the ideal-gas constant, and $T^{\prime}$ is the absolute temperature. In using equations 92 and 93 , the parameter most characteristic of the eddy-diffusion coefficient, $\epsilon$, is $D_{x}$, the longitudinal dispersion coefficient of the flow.

Regression analysis by Krenkel and Orlob (1963) of 58 laboratory measurements of $k_{2}$ made at depths from 0.08 to 0.20 foot in a 1-foot-wide flume yielded

$$
k_{2}=1.418 \exp \left[-E_{a} / R_{G} T^{\prime}\right] \frac{D_{x}}{H^{2}}-0.0069,
$$

where $E_{a}=5,875 \frac{\mathrm{cal}}{\mathrm{mole}}, R_{G}=1.99 \frac{\mathrm{cal}}{\mathrm{mole}^{\circ} \mathrm{K}}, T^{\prime}$ is the absolute temperature in degrees Kelvin, and the units of $k_{2}$ are reciprocal minutes. Because the procedure for measuring $D_{x}$ is difficult and time consuming, this parameter is not one of the commonly available stream-flow parameters. However, for uniform open-channel flows in the depth range given above, Krenkel (1960) showed by a regression analysis that $D_{x}$ can be related to the commonly measured mean flow parameters by

$$
D_{x}=6.425 E^{0.296} H^{1.243} \text {, }
$$

where $U$ is in feet per minute, $g$ is in feet per minute per minute, and $E$ is the energy dissipated per unit mass of flowing fluid. Alternatively, from a separate regression analysis of the same data, Krenkel (1960) and Krenkel and Orlob (1963) reported that $k_{2}$ at $20^{\circ} \mathrm{C}$ can be determined from

$$
\left(k_{2}\right)_{20^{\circ}}=1.141 \times 10^{-4} E^{0.408} H^{-0.66}
$$

where, again, $k_{2}$ is in reciprocal minutes. When equation 96 is to be used for flow at some temperature other than $20^{\circ} \mathrm{C}$, adjustment of $k_{2}$ must be made using a suitable temperature correction formula. (See eq 104.)

Experimental differentiation between equations 92 and 93 may be very difficult if $D_{i}=D_{m}$ because for a small temperature range (for example, $\pm 10^{\circ} \mathrm{C}$ )

$$
\frac{D_{m}}{\nu} \propto \exp \left(-E^{\prime} / T^{\prime}\right)
$$

where $E^{\prime}$ is a combination of the activation energies for molecular diffusion and viscous flow and is independent of temperature. Thus, a plot of $\log k_{2}$ versus $1 / T^{\prime}$ for constant $\epsilon / H^{2}$ will show a linear variation, whether molecular diffusivity or the activation energy for oxygen absorption is the controlling factor. Most likely, $D_{m}$ (or eq 92) controls, because equation 93 in effect states that interfacial resistance controls; but Davies and Rideal (1963) and Sherwood and Pigford (1952) stated that interfacial resistance is negligible for the absorption of slightly soluble gases.

An important factor to be considered when using equations such as 94 and 95 is that they were developed for flows in straight channels with uniform cross section, whereas natural streams generally have nonuniform cross sections and considerable curvature in plan. In such channels, $D_{x}$ can be considerably greater than the $D_{x}$ for an equivalent straight channel with uniform cross section, so equations 94 and 95 might not apply. Equations of the type of 96 might, however, be more valid in natural streams because the rate of energy dissipation includes the effects of nonuniform cross sections and curvature. Finally, as mentioned above, $D_{x}$ is difficult to measure or predict, whereas equations of the type of 96 use readily measurable mean-flow parameters. Thus, from this point of view, use of equation 96 would be recommended over the use of equation 94 .

\section{THACKSTON MODEL}

The Thackston model was presented by Thackston (1966) and Thackston and Krenkel (1969a), who postulated that

$$
k_{2} \propto \frac{f_{1}(s)}{f_{2}(H)},
$$


where $s$ is the average surface renewal rate and $H$ is the flow depth. They further postulated that $f_{1}(s)_{\propto} \epsilon_{y}$, where $\epsilon_{y}$ is the vertical eddy diffusivity at the water surface, and that $f_{2}(H) \propto H^{2}$. They assumed that

$$
\epsilon_{y} \propto \bar{\epsilon}_{y}=\frac{\kappa}{6} H u_{*},
$$

where $\overline{\boldsymbol{\epsilon}}_{y}$ is the average value of the vertical eddy diffusivity, $u_{*}$ is the shear velocity, $\sqrt{g H S}$, and $\kappa$ is von Karman's constant. The resulting prediction equation is of the form

$$
k_{2}=J \frac{u_{*}}{H},
$$

where $J$ is a constant to be determined from regression analysis.

Based on 40 values of $k_{2}$ measured by Thackston (1966) in a 2-foot-wide flume at flow depths ranging from 0.05 to 0.23 foot, a regression analysis yielded

$$
k_{2}=0.000215 \frac{u_{*}}{H}
$$

where $k_{2}$ is in reciprocal seconds, $u_{*}$ is in feet per second, and $H$ is in feet. More importantly, equation 101 fits rather well to the field data used by O'Connor and Dobbins (1958) and the data collected by Churchill, Elmore, and Buckingham (1962). Furthermore, the fit is improved if a Froudenumber relation is included in the regression analysis. A regression analysis which included these field data plus the flume data of Thackston (1966) yielded

$$
k_{2}=0.000125\left(1+\mathrm{F}^{0.5}\right) \frac{u_{*}}{H},
$$

where $F$ is the Froude number.

Some question exists as to the universal applicability of an equation of the type of 102 , because when the data of Krenkel (1960) were analyzed in the same fashion as those yielding equation 101, the prediction equation

$$
k_{2}=0.000469 \frac{u_{*}}{H}
$$

was obtained. Thackston and Krenkel (1969a) explained the difference between equations 101 and 103 by stating that because Krenkel's 1 -foot-flume data do not fit field data, whereas Thackston's 2foot-flume data do, the conditions in the 1-footflume were not really characteristic of field conditions. More likely, however, this discrepancy indicates that prediction equations of the type of 101 and 102 are not general enough and that some additional parameter such as the width-depth ratio, $W / H$, should be included in the prediction equation.

\section{EMPIRICAL PREDICTION EQUATIONS}

The empirical prediction equations result from least-squares multiple-regression analyses of the variables considered pertinent by the investigator or from dimensional analysis of the mean-flow and gas-liquid parameters. For water and air in the temperature range encountered in the field, it is generally assumed that the temperature dependency of $k_{2}$ resulting from the temperature dependence of the molecular diffusivity, kinematic viscosity, surface tension, and other physical properties can be embodied in a single function of the temperature. This dependency is generally removed before the least-squares analysis is begun. The empirical temperature-correction equation (the form of which can be derived from rate process considerations) is of the form

$$
K_{2_{T}}=K_{2_{20}} \theta^{\left(T-20^{\circ}\right)} \text {, }
$$

where $T$ is in degrees Celsius and the $\theta$ generally used is the 1.0241 suggested by Elmore and West (1961).

The empirical prediction equations are generally of the form

$$
k_{2}=\psi(\theta)^{T-20^{\circ}} \frac{U^{w}}{H^{j}}
$$

where $\psi$ is a constant obtained from a regression analysis of experimental data, and $w$ and $j$ may be chosen in this fashion; or $w$ and $j$ may be chosen for dimensional homogeneity of the prediction equation. Judgment of a particular prediction equation must be based on the quality of the data from which it is derived, and on how well it predicts field reaeration coefficients. The equations discussed in this section are based generally on fieldscale data and utilize readily measurable mean-flow parameters for prediction of reaeration coefficients. Presented in the following sections are equations by Streeter and Phelps (1925), Churchill, Elmore, and Buckingham (1962), Owens, Edwards, and Gibbs (1964), Langbein and Durum (1967), Isaacs and Gaudy (1968), and Negulescu and Rojanski (1969).

\section{STREETER-PHELPS EQUATIONS}

The pioneering study of reaeration coefficients of large-scale natural streams was presented by Streeter and Phelps (1925). In this study, the classical oxygen sag equation

$$
\frac{d D}{d t}=K_{1} L-K_{2} D
$$

was developed and was used in its integrated form to calculate reaeration coefficients from measured 
values of the time of travel, upstream and downstream values of the $D O$ deficit and $B O D$, and the BOD reaction rate constant, $K_{1}$.

The prediction equations were developed from data taken in 1914 and 1915 on the Ohio River between Pittsburgh, Pa., and Louisville, Ky. The study reach was 611 river miles long; it was separated into 11 sub-reaches for experimental measurements. During the study period at the various points in the study reach, the mean monthly values of discharge, depth, and velocity varied, respectively, from 1,670 to 309,000 cfs (cubic feet per second), from 16 to 48 feet, and from 0.1 to $4.6 \mathrm{fps}$ (feet per second). Eighty-six average values of $k_{2}$ were reported as corresponding to the monthly averages of the flow parameters existing over the period of measurement on the various sub-reaches.

The prediction equation suggested by Streeter and Phelps (1925) was

$$
\left(k_{2}\right)_{20^{\circ}}=\frac{Z U^{\eta}}{\left(H^{\prime}\right)^{2}}
$$

where $H^{\prime}$ is the depth above minimum low water stage and $k_{2}$ is in reciprocal days. The coefficient $Z$ is a function of what Streeter and Phelps called an irregularity factor, which is defined as the number of changes in channel bottom slope producing a change in elevation greater than 1 foot, expressed on a per-mile basis. For irregularity factors of 2.0 to $3.0, Z=\left[0.39\left(10^{1.16}\right) S+17\right]$, and for irregularity factors of 3.0 to $5.0, Z=1.1 S^{2.3}$, where $S$ is the channel water-surface slope, in feet per mile. The coefficient $\eta$ is a function of $\delta$, or $\eta=(\delta-0.17)$ / $(\delta-1.17)$, where $\delta$ is the mean relative increase in flow velocity per 5 -foot increase in gage height.

Because of the need for computing the irregularity factor and the coefficient $\delta$, equation 107 is difficult to use. Also, because $H^{\prime}$ is not a true streamflow parameter, a prediction equation in terms of this parameter probably is not completely general. Other authors (for example, O'Connor and Dobbins, 1958) have, however, reanalyzed some of the Streeter-Phelps data to yield prediction equations with a little more generality.

\section{CHURCHILL-ELMORE-BUCKINGHAM EQUATIONS}

Probably the best field study of the reaeration coefficient has been that by Churchill, Elmore, and Buckingham (1962). Their measurements were made in stretches below dams, where the water released was deficient in DO because of prolonged storage under conditions of thermal stratification, and where generally the BOD was negligible.

The study covered 16 different reaches on five rivers. Five hundred and nine individual $k_{2}$ deter- minations were made for 30 different mean-flow situations. The discharge, mean depth, and mean velocity ranged, respectively, from 952 to 17,270 cfs, from 2.12 to 11.41 feet, and from 1.85 to $5.00 \mathrm{fps}$.

Churchill, Elmore, and Buckingham (1962) performed many multiple-regression analyses on the pertinent stream flow, gas, and liquid parameters corresponding to the $k_{2}$ measurements. None of the prediction equations was found to be statistically any more significant than any of the others, so that the simplest one was suggested by these authors for general usage. This equation, which has a coefficient of multiple correlation of 0.82 , is

$$
k_{2}=5.026 U^{0.969} H^{-1.673}(1.0241)^{T-20^{\circ}} \text {, }
$$

where $k_{2}$ is in reciprocal days, $U$ is in feet per second, $H$ is in feet, and $T$ is in degrees Celsius.

Equation 108 is much more convenient to use than equation 107 because it utilizes only the readily available parameters $U, H$, and $T$.

\section{OWENS-EDWARDS-GIBBS EQUATION}

Owens, Edwards, and Gibbs (1964) reported reaeration-coefficient measurements for several English streams. These coefficients were determined using the disturbed-equilibrium method of deaeration with sodium sulfite and cobalt catalyst. Use of this procedure eliminates the necessity of considering parameters of the DO balance that are independent of the DO concentration.

The study by Owens, Edwards, and Gibbs (1964) included measurements on 21 different reaches of six streams. They listed 32 separate observed values of $k_{2}$ for stream discharges, depths, and mean velocities ranging, respectively, from 1.50 to $36.2 \mathrm{cfs}$, from 0.34 to 2.44 feet, and from 0.13 to $1.83 \mathrm{fps}$. They also listed six values of $k_{2}$ from Gameson, Truesdale, and Downing (1955) for two reaches of a different stream for discharges, depths, and mean velocities within the above ranges.

Multiple-regression analysis of the data by Owens, Edwards, and Gibbs (1964) yielded

$$
k_{2}=10.90 U^{0.73} H^{-1.75}(1.0241)^{T-20^{\circ}} \text {. }
$$

When Owens, Edwards, and Gibbs (1964) included the data of Gameson, Truesdale, and Downing (1955) and that of Churchill, Elmore, and Buckingham (1962) in the analysis, the resultant equation was

$$
k_{2}=9.41 U^{0.67} H^{-1.85}(1.0241)^{T-20^{\circ}} .
$$

In these equations, $k_{2}$ is in reciprocal days, $U$ is in feet per second, $H$ is in feet, and $T$ is in degrees Celsius. Equation 110 was obtained from $68 k_{2}$ values, which had a 300 -fold range from about 0.25 to 75 days $^{-1}$. Again, equations 109 and 110 are very 
convenient to use because they utilize only the readily available parameters $U, H$, and $T$.

\section{LANGBEIN-DURUM EQUATION}

Langbein and Durum (1967) combined the river data of O'Connor and Dobbins (1958) and of Churchill, Elmore, and Buckingham (1962) with the laboratory data of Krenkel and Orlob (1963) and of Streeter, Wright, and Kerr (1936) and obtained the equation

$$
\left(k_{2}\right)_{20^{\circ}}=3.3 \frac{U}{H^{1.33}},
$$

where $k_{2}$ is in reciprocal days, $U$ is in feet per second, and $H$ is in feet. They also considered in a general way the effect of downstream and regional variations of stream discharge on the reaeration coefficient computed from equation 111 . They found that the computed reaeration coefficients decreased in the downstream direction with about the square root of the discharge.

\section{ISAACS-GAUDY EQUATIONS}

Isaacs and Gaudy (1968) made a study of reaeration in a circular tank with moving walls intended to simulate streamflow conditions. They reported 52 individual $k_{2}$ observations for 20 different simulated streamflow conditions. The simulated depth ranged from 0.50 to 1.50 feet, and the simulated mean velocity, from 0.55 to $1.63 \mathrm{fps}$.

A multiple-regression analysis of the data by Isaacs and Gaudy (1968) yielded exponents of 1.0027 and -1.4859 on mean velocity and depth, respectively; so a second analysis was performed in which these exponents were held to 1.0 and -1.5 . The results of this analysis were

$$
k_{2}=3.053 \frac{U}{H^{3 / 2}}(1.0241)^{T-20^{\circ}} \text {. }
$$

Using 29 of the 30 data points of Churchill, Elmore, and Buckingham (1962), Isaacs and Gaudy obtained

$$
k_{2}=3.739 \frac{U}{H^{3 / 2}}(1.0241)^{T-20^{\circ}},
$$

but using Krenkel's (1960) flume data they obtained

$$
k_{2}=2.440 \frac{U}{H^{3 / 2}}(1.0241)^{T-20^{\circ}} .
$$

In equations 112,113 , and $114, k_{2}$ is in reciprocal days, $U$ is in feet per second, and $H$ is in feet.

Isaacs and Gaudy (1968) pointed out that the multiplicative constants in equations 112,113 , and 114 can be interpreted as

$$
\chi\left(\frac{D_{m}^{1 / 2}}{\nu^{1 / 6} g^{1 / 6}}\right)_{20^{\circ}},
$$

where the constant $\chi$ is dimensionless.
Equations of the type of 112 through 114 are convenient to use, and if the constant is expressed as in equation 115, they are dimensionally homogeneous. There is some question as to whether the data from which equation 112 is derived truly represent streamflow conditions (Thackston and Krenkel, 1969b; Bella, 1969), so that use of this equation for prediction of stream reaeration coefficients is questionable. However, equation 113 should be applicable to streamflows, at least for the range of flow parameters over which the measurements were taken, because it is based on field data. The disparity between the constants in equations 113 and 114 probably indicates the effects of various nonhomogeneities in the data collection and modeling procedures and also suggests that all the pertinent variables have not been included in the prediction equations.

\section{NEGULESCU-ROJANSKI EQUATIONS}

The equations given by Negulescu and Rojanski (1969) were derived by regression analysis from a series of $18 k_{2}$ and $D_{x}$ measurements in a 66-footlong by 0.66 -foot-wide recirculating flume. Not all the data are given, but for those available, the depths range from 0.164 to 0.492 foot, and the velocities range from 0.656 to $1.903 \mathrm{fps}$. The equations obtained by Negulescu and Rojanski are

$$
k_{2}=4.74\left(\frac{U}{H}\right)^{0.85} \text {, }
$$

and

$$
k_{2}=1.423 D_{x}\left(\frac{U}{H}\right)^{1.63},
$$

where $k_{2}$ is in reciprocal days, $U / H$ is in reciprocal seconds, and $D_{x}$ is in square feet per day. Comparing values of $k_{2}$ predicted by equations 116 and 117 and values from a set of river measurements (which are not reported), Negulescu and Rojanski (1969) concluded that equation 117 is the more accurate.

Again, from the standpoint of convenience in obtaining measured parameters for prediction of $k_{2}$ in field streams, equation 116 is the desirable one to use.

\section{EXPERIMENTAL DETERMINATION OF THE REAERATION COEFFICIENT}

All the experimental techniques for determining the reaeration coefficient in open-channel flow, except the tracer technique of Tsivoglou (1967) and Tsivoglou and others $(1965,1968)$, use some integrated form of a DO balance equation - for example, an integrated form of equation 12 . The determination of $K_{2}$ requires, therefore, that all 
other parameters in the equation be measured. These parameters are determined by using a variety of techniques, all of which depend on the measurement of the amount of oxygen dissolved in a volume of water.

The purpose of this section is to discuss (1) the basic DO measurement techniques, (2) the various techniques for determining $K_{2}$, and (3) an analysis of the errors expected from the different techniques for determining the reaeration coefficient.

\section{MEASUREMENT OF DISSOLVED OXYGEN IN WATER}

The basic method for the determination of DO in water is the Winkler procedure, which was first described in the literature in 1888. (See Am. Public Health Assoc., 1965, p. 414, for reference.) Since that time, the procedure has been improved by advances in equipment, technique, and instrumentation, and it is still the basis for most analytical methods for the determination of DO in water.

Oxidizing or reducing substances in the sample interfere with the procedure, however, and various modifications of the basic Winkler method have been developed. A common interference in waters receiving sewage and effluents from sewage treatment plants is nitrate nitrogen, and the Alsterberg modification is used under these conditions. Details of the procedure were given by the American Public Health Association (1965) and by Rainwater and Thatcher (1960). The Alsterberg modification is the procedure generally used for routine analysis of natural waters. Details of other modifications of the basic Winkler procedure were also given by the American Public Health Association (1965), and several of these modifications were compared by Montgomery, Thom, and Cockburn (1964).

Various instrumental methods for the determination of DO in water have also been developed. These include, for example, gas-exchange, gaschromatographic, radiometric, voltammetric, and membrane-electrode methods. The procedures used in these methods and the advantages and disadvantages of these methods and of titrimetric methods were discussed by Mancy and Jaffe (1966).

\section{TECHNIQUES FOR DETERMINING THE REAERATION COEFFICIENT}

Three general techniques are used for determining the reaeration coefficient of open-channel flow. These are the dissolved-oxygen balance technique, the disturbed-equilibrium technique, and the tracer technique. The first two techniques utilize an integrated form of the equation of non- dispersive conservation of mass for a gas dissolved in a liquid (eq 28 with $\bar{\epsilon}_{x}$ negligibly small). The third technique utilizes an integrated form of this equation for an instantaneous input of tracer material which is convected and dispersed longitudinally by the flow. The basis and procedures of these techniques are discussed in the following paragraphs.

\section{DISSOLVED-OXYGEN BALANCE TECHNIQUE}

Studies utilizing the DO balance technique are of two types: those in recirculating flumes, and those in natural streams or in nonrecirculating flumes. Studies of the first type utilize the nonsteady-state nondispersive form of the conservation of mass equation, equation 28 with $\bar{\epsilon}_{x}=0$. Those of the second type utilize the steady-state nondispersive form of this equation - that is, equation 28 with the $\bar{\epsilon}_{x}$ and the $\partial \bar{C} / \partial t$ terms equal to zero. We know of no techniques which have been applied to the calculation of $K_{2}$ in nonsteady dispersive conditions; however, the numerical techniques of Thomann (1963, 1965) and Dresnack and Dobbins (1968) could be modified to compute $K_{2}$ from measured variations of DO with time.

The general DO balance technique for natural streams is based on a more sophisticated version of the equations originally presented by Streeter and Phelps (1925) - for example, equations 12 and 13, as presented by Camp (1963). These onedimensional steady-state equations are applicable to conditions which meet the assumptions previously given for equations 9 and 10 . In addition, longitudinal dispersion is generally assumed to have a negligible effect on the longitudinal DO profiles in natural streams.

With these assumptions, the solutions to equations 12 and 13 yield the BOD and the DO deficit at a downstream station, $x=x_{\mathrm{b}}$, in terms of the BOD and DO deficit at an upstream station, $x=x_{\mathrm{a}}$. The solutions are

$$
L_{\mathrm{b}}=\left[L_{\mathrm{a}}-\frac{m}{2.3\left(k_{1}+k_{3}\right)}\right] 10^{-\left(k_{1}+k_{3}\right) t}+\frac{m}{2.3\left(k_{1}+k_{3}\right)},
$$

and

$$
\begin{aligned}
D_{\mathrm{b}} & =\frac{k_{1}}{k_{2}-k_{1}-k_{3}}\left[L_{\mathrm{a}}-\frac{m}{2.3\left(k_{1}+k_{3}\right)}\right]\left[10^{-\left(k_{1}+k_{3}\right) t}-10^{-k_{2} t}\right]+ \\
& \frac{k_{1}}{k_{2}}\left[\frac{m}{2.3\left(k_{1}+k_{3}\right)}-\frac{p}{2.3 k_{1}}\right]\left(1-10^{-k_{2} t}\right)+D_{\mathrm{a}} 10^{-k_{2} t,}(119)
\end{aligned}
$$

where the flow time is $t=\frac{x_{\mathrm{b}}-x_{\mathrm{a}}}{U}$, the rate constants are to the base 10 , and equation 12 has been solved in deficit form. 
Equation 119, in its most general form, must be solved for $k_{2}$ by trial and error. Before this can be done, however, the other parameters appearing in equations 118 and 119 must be evaluated. The techniques used in evaluating these parameters are discussed briefly in the following paragraphs.

$$
\text { BOD TEST AND } K_{1}
$$

Of the various parameters in a DO balance, the rate constant, $K_{1}$, for the oxidation of organic wastes probably has been studied to the greatest extent. The oxidation process is generally considered to be a first-order process as represented by equation 1 . The two parameters in equation 1 , $K_{1}$ and $L$, may be determined from a BOD test. The earliest recorded use of a BOD test was in 1870 (Theriault, 1926). Since that time, numerous studies have been made of the BOD test. The literature prior to 1927 on the BOD test was summarized by Theriault (1927), and a bibliography of the literature on the BOD test since 1927 was compiled by Hull (1959).

The BOD test consists basically of obtaining two samples of the water containing organic wastes, determining the DO concentration in one of the samples at the beginning of the test, incubating the second sample in darkness at $20^{\circ} \mathrm{C}$ for a period of time, usually 5 days, and determining the DO concentration in the second sample at the end of the incubation period. The difference in DO concentrations is the 5-day BOD of the sample.

In practice, however, the procedure is not so simple. To prevent complete depletion of the DO in the test sample, the sample must be properly diluted before the start of the test. The dilution water must be properly prepared and seeded. The type and amount of seed to be used in the dilution water for the particular waste of interest must be determined by experience. If the sample is alkaline or acidic or contains residual chlorine or other toxic substances, then it also must be treated before the start of the test. The complete details of the standard 5 -day, $20^{\circ} \mathrm{C}$ BOD test were given by the American Public Health Association (1965).

The reaeration technique of Elmore (1955) for BOD determinations has the advantage that dilution water and seeding are not required. This procedure, however, has the disadvantages that the 5 -day BOD must be less than $25 \mathrm{mg} / \mathrm{l}$, large volumes of sample are required, and considerable attention on the part of the technician is necessary during the initial stages of the test.

A respirometer assembly for BOD determinations was described by Gannon, Pelton, and
Westfield (1965). The sample is placed in a sealed cell equipped with a DO measurement probe, and the DO concentration is measured continuously. When the DO concentration decreases to $2 \mathrm{mg} / \mathrm{l}$, the cell is aerated with compressed air. This process is continued for as long as desired. The respirometer has the same advantages as the reaeration technique in that no dilution and seeding are necessary, and it has the additional advantage that the original sample is kept intact. The literature on other types of improved techniques for BOD determinations was reviewed by Gannon, Pelton, and Westfield (1965), and a complete review of respirometric methods was presented by Montgomery (1967).

The results of a BOD test are usually in the form of either BOD or amount of waste oxidized at time $t$ as a function of $t$. The applicable equation is

$$
X_{t}=L-L 10^{-k_{1} t} \text {, }
$$

where $X_{t}$ is either the BOD or the amount of waste oxidized up to time $t$, and $L$ is the total first stage, or carbonaceous, BOD of the sample. Because both $L$ and $k_{1}$ are unknown, several different techniques have been developed for determining $L$ and $k_{1}$ from the results of BOD tests. Among these techniques are the least-squares procedure of Reed and Theriault (1931), the log-difference method of Fair (1936), the slope method of Thomas (1937), the moments method of Moore, Thomas, and Snow (1950), the graphical procedure of Lee (1951), and the ratio methods of Williams (1951) and Sheehy (1960). The Reed-Theriault procedure is the most accurate, but it also requires the most time for the calculations because it is a trial-and-error procedure. Of the other procedures, the moments method gives the most accurate results for the time consumed (Ludzack and others, 1953; Schroepfer and others, 1960; Gannon, 1966; Zanoni, 1967).

The extensive series of BOD measurements by Streeter and Phelps (1925) suggested that $k_{1}$ has a value of $0.1 \mathrm{day}^{-1}$ at $20^{\circ} \mathrm{C}$ for all types of sewage and organic wastes. Later work, however, has shown that $k_{1}$ at $20^{\circ} \mathrm{C}$ may range from about 0.01 $\mathrm{day}^{-1}$ for some industrial wastes and well-oxidized treatment-plant effluents to about $0.30 \mathrm{day}^{-1}$ for readily oxidizable wastes (Camp, 1963, p. 247). Schroepfer, Robins, and Susag (1960) found that the $k_{1}$ values for raw wastewaters and primary settled effluents from treatment plants in the Minneapolis-St. Paul area varied from $0.14 \mathrm{day}^{-1}$ on Sundays and holidays to $0.24 \mathrm{day}^{-1}$ on weekdays. They concluded that $k_{1}$ depended greatly on the degree of stabilization of the effluent. Kothandaraman and Ewing (1969) found that $k_{1}$ values for 
the Ohio River downstream of Cincinnati, Ohio, were normally distributed and had a mean value of $0.075 \mathrm{day}^{-1}$ and a standard deviation of 0.028 day $^{-1}$. Camp (1965) also noted considerable daily variation of $k_{1}$ values for several stations on the Merrimack River in Massachusetts. Thus, $k_{1}$ apparently cannot be considered a constant, but $k_{1}$ values must be determined for the waste, the reach, and the time of interest.

The $k_{1}$ values determined at $20^{\circ} \mathrm{C}$ in the laboratory are converted to the appropriate river temperature by means of the equation

$$
\left(k_{1}\right)_{T}=\left(k_{1}\right)_{20^{\circ}} \theta^{\left(T-20^{\circ}\right)} \text {, }
$$

where $\theta$ is the temperature coefficient, which has a value of 1.047 for temperatures from $10^{\circ}$ to $37^{\circ} \mathrm{C}$ (Camp, 1963, p. 247). Zanoni (1967) found the same value for $\theta$ for a somewhat smaller temperature range $\left(15^{\circ}\right.$ to $\left.32^{\circ} \mathrm{C}\right)$ and $\theta$ values of 0.985 for temperatures from $32^{\circ}$ to $40^{\circ} \mathrm{C}$ and 1.126 for temperatures from $2^{\circ}$ to $15^{\circ} \mathrm{C}$. He also found that $L$ increased with temperature.

Widespread use of the laboratory 5-day $20^{\circ} \mathrm{C}$ BOD test has made apparent several aspects of the standard BOD bottle test, as well as the aforementioned problems with dilution and seeding, that require additional study. These aspects are concerned with how well the diluted and seeded sample incubated in darkness under quiescent conditions in a constant temperature bath approximates the behavior of a slug of water of similar size under dynamic streamflow conditions. For example, the turbulence conditions are obviously different, and increased values of $k_{1}$ have been found when the BOD bottles were stirred or rotated (Owens and Edwards, 1966; Gannon, 1966). The increased turbulence prevents deposition of suspended material and facilitates the transport of the wastes to the organisms. The large surface-tovolume ratio of the bottle may result in increased growth of organisms on the inside of the bottle and, hence, faster DO consumption than would occur in an unconfined slug of water of the same size (Symons, 1966; Hull, 1969). The bottle test does not allow for the possible decrease in the respiration rate of organisms with decreasing DO concentration in the bottle (Owens and Edwards, 1966). When algae are present, the bottle test conducted in darkness could give a high value for the BOD of the sample because of respiration demands of the algae (Owens and Edwards, 1966). In the stream, however, the algae replenish the oxygen consumed by their respiration by the process of photosynthesis during daylight hours. The 5-day period required for the standard bottle test is also a dis- advantage because the conditions in the streams may change considerably while the $k_{1}$ value is being determined. Camp (1965) found that the water temperatures varied widely during the daylight hours on the Merrimack River in Massachusetts and concluded that it was pointless to determine the BOD in the laboratory at a constant temperature. Hull (1966, 1969) and Camp (1965) recommended using a dark bottle suspended in the stream at the point at which the sample was obtained. This procedure eliminates delay and the need for a temperature correction, and it gives a BOD value for the period of sample incubation. To determine $k_{1}$, however, requires BOD values for different times or continuous recording of the DO concentration in the submerged bottle, and one is again faced with the problem of changing conditions in the stream.

BOTTOM DEPOSITS, $K_{3}$, AND $m$

In addition to the biochemical oxidation of organic wastes in the flowing stream, wastes may also be removed from the stream by settling to the bottom. Bottom deposits form in three general ways (Velz, 1958): (1) deposition of settleable solids from the wastes; (2) deposition resulting from the flocculation or coagulation of solids within the stream; and (3) biological extraction and accumulation of wastes by growths attached to the bottom of the stream.

The first type of deposit is the result strictly of a sedimentation process. The second type may be considered as resulting from a process analogous to an activated sludge system in which the growth is dispersed throughout the flowing stream. Under certain conditions, the growths flocculate, and the flocs increase in size until sedimentation occurs. The third type of deposit may be considered as resulting from a process analogous to that occurring in a trickling filter. Biological extraction and accumulation of wastes were studied by Velz and Gannon (1963). The rate of sedimentation is generally assumed to be proportional to the amount of BOD, or

$$
\text { rate of sedimentation }=K_{3} L \text {. }
$$

A velocity of $0.6 \mathrm{fps}$ was considered by Velz (1958) to be the critical velocity at which organic wastes begin to deposit.

Even though the formation of a bottom deposit may result in a reduction of the BOD of the water from which the waste was removed, the deposit may be a sink for DO to other masses of water 
passing over the deposit. Several processes may be involved:

1. BOD additions to the overlying water as a result of resuspension of the bottom deposits. A velocity of 1.0 to $1.5 \mathrm{fps}$ is considered necessary for resuspension, the actual velocity necessary being dependent upon the degree of decomposition and compaction of the deposit (Velz, (1958).

2. Diffusion of the anaerobic decomposition products of the deposit into the overlying water.

3. Diffusion of DO from the overlying water into the aerobic zone of the deposit.

Fair, Moore, and Thomas (1941) concluded that the oxygen demand of sludge deposits was controlled by process 2 and that process 3 was relatively unimportant. The relative importance of processes 1 and 2 may be seen qualitatively from an experiment of Edwards and Rolley (1965) in which the DO consumption rate of a mud sample increased approximately sixfold when the sample began to scour. Similarly Brown and Clark (1968) reported large decreases in the DO concentrations during dredging operations in a tidal estuary. They also pointed out that resuspension of bottom deposits decreases light penetration and, consequently, the additions of DO by photosynthesis.

The importance of bottom deposits on DO balances has been demonstrated by several field studies, including those by Velz (1958), Owens and Edwards (1963), and Camp (1965). Velz (1958) discussed the effect of sludge deposits on DO profiles for several situations. Owens and Edwards (1963) found on the River Lark in England that they obtained very poor agreement between measured and observed DO profiles until the DO demands of the mud were included in the DO balance. Edwards and Owens (1965, fig. 1) presented the percentage of the total DO consumed by mud as a function of the water depth for BOD and mud consumption values typical of English streams. Their graph shows that for depths of flow less than 1.25 meters, the mud consumed more oxygen than was consumed by the biochemical oxidation of the wastes in the water. Camp (1965) found on the Merrimack River in Massachusetts that 50 to 80 percent of the BOD removal was by sedimentation. He also noted that most of the BOD removed by sedimentation consisted of solids formed by bioflocculation.

The effects of removal of BOD by sedimentation and addition of BOD to the overlying water by bottom deposits have been included in the DO balances of Dobbins (1964b) and Camp (1963). Dobbins (1964b) described three situations for which $K_{3}$ can be determined if $K_{1}$ has been determined from laboratory measurements. The term $D_{B}$, which includes the addition of BOD to the water from bottom deposits, in general must be determined by difference from the DO profile equation. This procedure, however, requires an independent estimate of the reaeration coefficient, $K_{2}$. Camp (1965) recommended that $m$, the rate of addition of BOD to the overlying water, be determined from in situ measurements by the technique of Fair, Moore, and Thomas (1941) and Camp (1963, p. 310), and that $K_{3}$ be determined from the BOD profile equation after $K_{1}$ has been determined from laboratory BOD tests.

In addition to the studies of the overall effect of bottom deposits on the DO and BOD balances, the consumption of DO by muds has been studied in some detail with somewhat contradictory results. For example, Baity (1938) and Fair, Moore, and Thomas (1941) found that the DO consumption rate, $\omega$, was dependent on the depth, $d_{M}$, of the deposit according to

$$
\omega=\mathrm{a}_{1} d_{M}^{0.485}
$$

in which $a_{1}$ is a constant. On the other hand, Edwards and Rolley (1965) found that the DO consumption rate was independent of the deposit depth for depths greater than $2 \mathrm{~cm}$, and McDonnell and Hall (1969) concluded that the consumption rate was essentially independent of the sample depth.

With respect to the dependence of the DO consumption rate on the DO concentration of the overlying water, Baity (1938) found that the consumption rate was independent of the DO concentration for the range of 2 to $5 \mathrm{mg} / \mathrm{l}$ for deposits 0.5 $\mathrm{cm}$ thick. Edwards and Rolley (1965), however, found that the DO consumption rate was dependent on the DO concentration in the overlying water according to

$$
\omega=\mathbf{a}_{2} C^{b_{1}},
$$

where $b_{1}$ is 0.45 for the muds studied. This relation was valid for DO concentrations in the range from 2 to $8 \mathrm{mg} / \mathrm{l}$ but was not satisfactory for muds containing large populations of midge larvae. Similar results were obtained by McDonnell and Hall (1969).

An attempt by Rolley and Owens (1967) to correlate the DO consumption rates with various chemical properties of the muds was unsuccessful. They attempted to correlate five chemical properties with DO consumption rates for both winter and summer samples from 12 English rivers but found no correlation, although a strong correlation was found among all the chemical properties. 
PHOTOSYNTHESIS AND RESPIRATION

The presence of phytoplankton, benthic algae, and rooted aquatic plants in a stream or river results in the production of oxygen through the process of photosynthesis. The plants combine carbon dioxide and water in the presence of sunlight to produce carbohydrates and oxygen. At the same time, however, the plants require oxygen in their respiration process. In general, the rate of production of oxygen exceeds the rate of consumption during daylight hours whereas consumption is predominant during nighttime hours. This may result in a pronounced diurnal variation of the DO concentration in the stream, the maximum DO concentration occurring in the afternoon and the minimum DO concentration occurring just before dawn.

In the past, the photosynthesis and respiration processes have been of interest only to biologists, who in their studies attempted in a very general way to allow for additions of DO by reaeration. Engineers, on the other hand, with the exception of a few such as Hull (1960), ignored photosynthesis and concentrated their efforts on the reaeration process. When photosynthesis is ignored, grossly incorrect values of the reaeration coefficient, $K_{2}$, may be obtained if the usual computation procedure is used. For example, Gunnerson and Bailey (1963) found negative values of $K_{2}$ for two sampling periods on the Sacramento River, Calif. Lynch (1965) also found negative $K_{2}$ values for nocturnal sampling periods on the Tioughnioga River in New York, and he believed that the computed $K_{2}$ values for daytime sampling periods would be larger than the true values if a separate factor for photosynthesis were not included in the DO balance. Similarly, Camp (1965) concluded that the $K_{2}$ values for streams of the type of the Merrimack River were considerably smaller than had been previously thought because of including photosynthetic effects with reaeration.

Opinions as to whether the DO produced by photosynthesis is an asset or a liability to a stream vary widely. Camp (1963) stated that the DO produced by photosynthesis should not be relied upon because there is no assurance that this DO will be available during the critical low-flow periods of summer when it is needed most. Similarly, Imhoff and Fair (1956) suggested that the DO produced by photosynthesis should not be relied upon in engineering calculations because it is available only during daylight hours and the warmer seasons of the year. On the other hand, Camp (1965) found in a summer study on the Merrimack River in Massachusetts that about 67 percent of the DO was furnished by photosynthesis and that reaeration was relatively unimportant, and he concluded "that reliance must be placed on the photosynthetic production of DO." Merritt, McDonald, and Paulson (1968) found on the Iowa River that photosynthesis was a contributing factor in the DO balance, and they concluded that "whether or not the photosynthetic effect can be relied upon quantitatively, it remains an important factor of the oxygen balance in streams." Similar photosynthetic effects have been observed in European rivers, and this literature has been summarized by Edwards and Owens (1965). Leffel (1968) found on the Chao Phraya River in Thailand that photosynthetic production of DO was small in comparison with reaeration. Bain (1968) stated that in a deep turbid stream the photosynthetic production of DO often is not sufficient to offset the respiration demands of the plants, even if the stream is well mixed. O'Connell and Thomas (1965) pointed out that if the photosynthetic production of DO is by benthic algae or rooted aquatic plants rather than phytoplankton, then photosynthesis is a liability because the oxygen produced during daylight hours by the attached plants is not in the same mass of water from which oxygen is consumed by nighttime respiration. An example of this cited by O'Connell and Thomas is the shallow rapidly-flowing Truckee River below the outfall of the sewage treatment plant of Reno, Nev. In a deep slowly-flowing stream, the response of the stream to a nutrient source is the development of phytoplankton which move with the stream. Hence, the production and consumption of oxygen occur at different points in space but in essentially the same mass of water. In streams with attached plants, the production and consumption of oxygen occur at the same point in space but in different masses of water. Edwards (1962) suggested that sometimes plant growths may be beneficial even when there is no net gain of DO because the plants produce oxygen during the summer conditions of low flows and high temperatures and remove oxygen during the decay process in winter when high flows and low temperatures exist. Gunnerson (1966) suggested that photosynthesis may be neglected if the time period over which the data are averaged is sufficiently long - for example, longer than 1 year.

Obviously, then, considerable controversy exists concerning photosynthesis and respiration in a natural stream. Hull (1966) pointed out, however, 
that the controversy generally is whether the oxygen production by photosynthesis is significant and not whether a term for photosynthesis should be included in the DO balance. Thus Camp (1963), Dobbins (1964b), the Water Pollution Research Laboratory group (Edwards and Owens, 1962, 1965; Edwards, 1962; Owens, 1965), O'Connor (1967), and others have included terms for the photosynthetic production of oxygen in their DO balances.

The production of oxygen by plant photosynthesis depends on several factors, including light intensity at the water surface, optical density of the water, and weight and distribution of plants in the stream (Owens and Edwards, 1964). The depth of the stream affects the photosynthetic production rate in that the extent of light penetration varies inversely with depth. Photosynthesis is limited to the euphotic zone, which is generally assumed to extend to the depth at which the light intensity is 1 percent of the light intensity at the surface (Owens and Edwards, 1966). The optical density of the water depends on the concentration, type, and size of suspended particles and on the color of the water produced by dissolved materials. An example of a situation where the environmental conditions were suitable for a high level of photosynthetic oxygen production but where actual production was limited to a very low level because of the turbidity of the water is the Chao Phya River in Thailand (Pescod, 1969). The weight and the distribution of the plants affect the net DO production mainly by affecting the total respiration rate. Thus, as the plant density increases, the photosynthetic production rate remains approximately constant because of selfshading by the plants, although the respiration rate continues to increase (Edwards, 1968).

Several procedures have been developed for the measurement of photosynthetic and respiration effects in natural streams. These include the upstream-downstream method (Odum, 1956; Owens, 1965), the single-curve procedure (Odum, 1956), which is a simplification of the upstreamdownstream procedure; and the light- and darkbottle technique.

The light- and dark-bottle technique requires collection of water samples at a number of verticals in the cross section and at a number of points at each vertical so that the number of samples is sufficient to yield an accurate value of photosynthesis and respiration for the cross section. Each sample is divided into three parts; one part is placed in a transparent glass bottle, another part is placed in an opaque bottle so that all light is excluded, and the third part is analyzed for the DO concentration. The light and dark bottles are suspended in the stream at the sampling point for a period of time, usually 24 hours or a multiple thereof. At the end of the desired period, the light and dark bottles are analyzed for their DO concentrations. Equations for the light-bottle and darkbottle DO concentration are, respectively,

$$
\begin{gathered}
C_{l f}-C_{l i}=P_{l}-R_{l} \\
C_{d i}-C_{d f}=R_{d},
\end{gathered}
$$

where the $l$ and $d$ subscripts refer to light and dark bottles, respectively; $i$ and $f$ are subscripts referring to initial and final DO concentrations; $R$ is the gross respiration, or BOD; $C$ is DO concentration; and $P$ is the photosynthetic oxygen production. For reasonably short periods of time, $R_{l}=R_{d}$, and because $C_{l i}=C_{d i}$, it follows that

$$
C_{l f}-C_{d f}=P_{l} \text {. }
$$

Hull (1963) believed that the light- and dark-bottle technique probably is more accurate than the standard BOD test and that the technique in general gives conservatively low estimates of the oxygen production by photosynthesis.

Symons (1966) discussed several problems with the classical light- and dark-bottle technique, including unnaturally large surface-to-volume ratios in the bottles, the relatively long time required for the test, the lack of mixing in the bottles, and the necessity for removal of samples from the stream, which may result in harm to the algae. He discussed a three-probe chamber device designed to eliminate these problems. O'Connell and Thomas (1965) also used a chamber device but did not measure DO continuously.

The upstream-downstream method uses measurements of the DO concentrations at the ends of the reach of interest for a 24 -hour period. The basis of the procedure is a DO balance of the form (Odum, 1956)

$$
Q_{0}=P-R+D_{0}+A_{0},
$$

where $Q_{0}$ is the rate of change of DO content, $P$ is the gross primary production, $R$ is the respiration rate, $D_{0}$ is the rate of reaeration, and $A_{0}$ is the rate of addition of $\mathrm{DO}$ to the reach by drainage accrual. Each term of equation 128 is on an area basis - for example, in units of grams per square meter per hour.

If each term in equation 128 is divided by $H$, the mean depth of flow, the result is

$$
q=p-r+d_{0}+a_{0},
$$

where the terms are now on a concentration basis - for example, in units of milligrams per liter per day.

The gross primary production, $P$, is defined as 
the sum of the net DO plant production and community respiration during the daylight hours. Therefore, it follows that if $r$ and $d_{0}$ are independent of time and $a_{0}$ is negligible or independent of time (Odum, 1956),

$$
P=H \int_{t_{1}}^{t_{2}}\left(q-q_{1}\right) d t=\frac{F}{A_{s}} \int_{t_{1}}^{t_{2}}\left(\Delta C-\Delta C_{1}\right) d t,
$$

where $F$ is the volumetric water flow rate, $A_{s}$ is the surface area of the reach, $\Delta C$ is the DO concentration difference between the upstream and downstream stations, and the subscripts 1 and 2 refer, respectively, to the time at which sunrise occurred and the time in the evening at which $q$ or $\Delta C$ returned to its sunrise value. When equation 130 is used to determine $P$, equation 128 must have units of weight per unit of area per day.

In general, the oxygen supplied by reaeration, $d_{0}$, is not independent of time because the rate of reaeration depends on the DO deficit. If a reaeration coefficient is available for the reach, then a correction can be applied for each hour in the calculation of the $q$ curve from the DO measurements.

The reaeration coefficient can be calculated from the diurnal DO measurements if $r$ and $a_{0}$ are assumed independent of time. From measurements before dawn and in the evening, when the $p$ values are zero, it can be shown (Odum, 1956) that

$$
K_{2}=\frac{q_{M}-q_{E}}{D_{M}-D_{E}}
$$

where $D$ is now the average $D O$ deficit in the reach, and the $M$ and $E$ subscripts refer to the morning and evening times, respectively. The reaeration coefficient calculated from equation 131 is the $K_{2}$ for the average deficit of the reach as compared with an integral $K_{2}$ obtained by integrating the deficit over the DO change.

Both equations 130 and 131 assume that $r$ is independent of time and, hence, independent of the DO concentration level. Opinions differ as to the validity of this assumption. Odum (1956) concluded on the basis of the available information that it was simplest to assume $r$ constant. On the other hand, Edwards, Owens, and Gibbs (1961) concluded that the respiration rate of plants depended on the DO concentration level. Therefore, corrections for the dependence of $r$ on DO concentration may be necessary.

The respiration rate can be determined from the results of an upstream-downstream experiment in the following ways (Odum, 1956). First, if the rate of reaeration is known and $A_{0}$ is negligible, then for the hours of darkness the respiration rate can be calculated from the observed rate of change of DO in the reach. Second, if during the hours of darkness the water is saturated with DO at some time, as usually occurs after sunset, then at that instant, because the reaeration is zero, the respiration rate is equal to the rate of change of $D O$ in the reach, assuming $A_{0}$ is negligible.

The single-curve procedure (Odum, 1956) is a simplification of the upstream-downstream procedure in which the diurnal variation of DO concentration at a single station is used in the calculation of $P$ and $R$. This procedure assumes that the entire reach experiences uniform changes in the DO concentration, and hence the curves at the upstream and downstream stations would be identical. Owens (1965) compared the two calculation procedures for two stations on the River Ivel in England which had nearly identical diurnal DO curves and found that the calculated $P$ and $R$ values were different for the two procedures and also different for the two stations. Edwards and Owens (1962) also found differences between the two procedures.

In addition to their direct effects on the DO balance, plants also exert indirect effects on the DO balance. For example, heavy plant growths can increase the depth of flow and reduce the water velocity, which in turn reduces the addition of oxygen by reaeration. At the same time, the reduced velocity increases the deposition of solids. If the plants extend through the surface, then turbulence at the air-water interface may be increased, thereby facilitating the absorption of oxygen by the water. Finally, the presence of the plants increases the surface area on which the bacteria can live and oxidize wastes present in the stream.

\section{OTHER PARAMETERS}

Other parameters that are necessary in a DO balance study include the DO concentrations at the upstream and downstream ends of the study reach and the flow time through the reach.

The flow time can be determined from mean velocity measurements and the distance between the ends of the study reach, or

$$
\Delta t=\frac{\Delta x}{U} \text {. }
$$

Distances may be obtained from topographic maps, and mean velocities from current meters or from stage-discharge ratings and cross-section measurements. Long study reaches are desirable; however, practical considerations such as variations in the channel cross section, location of sewage outfalls, and personnel requirements generally limit the length of reach that can be studied. 
The flow time can also be determined from tracer measurements, using either radioactive or fluorescent tracers. The procedure consists of instantaneously injecting a quantity of the tracer far enough upstream from the reach of interest so that the tracer can be uniformly mixed in both the lateral and vertical directions before it enters the reach. Procedures for estimating the distances required for complete mixing have been presented by Sayre (1965) and Fischer (1967). The flow time is the time required for the tracer cloud to move through the study reach. In some instances, the peaks of the tracer concentration versus time curves have been used in flow-time determinations; however, it is more correct to use the centroids of the curves. The flow time is the difference between the times at which the centroid of the dye cloud passes the downstream and upstream ends of the reach.

The upstream and downstream DO concentrations are determined from samples obtained at the ends of the study reach. One of the procedures previously described is used to determine the DO concentrations of the samples. The samples at the downstream point usually are obtained at a time lag of $\Delta x / U$ after the samples at the upstream point. This is done because DO balance equations generally are written in a coordinate system moving with the mean flow velocity.

The number of samples obtained at each cross section must be sufficient to define a mean DO concentration for the cross section. As mentioned previously, gradients of DO in the vertical direction in a flowing stream are generally negligible. The lateral DO distribution depends on many factors, including distance from sewage outfalls and stream tributaries, cross-section shape and tortuosity of the channel, and size, shape, and spacing of bed roughness elements. Churchill, Elmore, and Buckingham (1962) observed consistent U-shaped DO distributions across the stream channel, and they attributed these to (1) incomplete mixing of tributary inflows, (2) increased reaeration near the banks because of the longer traveltime, and (3) unequal effects across the stream width of photosynthesis and respiration by attached aquatics. Thus the possibility of lateral variations in the DO concentration should be considered.

To determine $k_{2}$ in the most general field situations, all the parameters in equation 119 must be evaluated using the methods previously described. In specific reaches, however, some of the factors in equation 119 may be of negligible importance. For example, if $p, m$, and $k_{3}$ are negligible, equation
119 reduces to the classical Streeter-Phelps sag equation, equation 6. Examples of reaches in which $p, m$, and $k_{3}$ were not negligible have been given previously. In the reaches chosen specifically for the study of reaeration by Churchill, Elmore, and Buckingham (1962), the BOD, $k_{3}, m$, and $p$ parameters were all negligible, so that equation 119 reduced to

$$
D_{\mathrm{b}}=D_{\mathrm{a}} 10^{-k_{2} t}
$$

Thus, $k_{2}$ could be calculated simply from DO measurements on samples obtained at two points in the reach separated by the flow time, $t$. In general, however, equation 119 or a similar equation should be the starting point in a DO balance calculation of $k_{2}$ for a natural stream, and parameters should be eliminated only after they have been shown to be negligible for the reach and hydraulic conditions of interest.

\section{CALCULATION OF $k_{2}$ IN A RECIRCULATING FLUME}

In a laboratory flume the sources and sinks of DO can be controlled so that the BOD, $k_{3}, m$, and $p$ parameters will in general be zero. With these conditions, the reaeration coefficient can be calculated from equation 133. Flume lengths, however, are limited by space and economical considerations so that the available flow times and the consequent change in DO deficit generally will be very small. Thus the calculated reaeration coefficients will be subject to large errors. This problem can be alleviated by recirculating the water through the flume.

In recirculating flumes, the water is partly deaerated before the start of an experiment, usually using sodium sulfite in the presence of a cobalt catalyst. As the water circulates through the flume, it reaerates at a rate which is dependent on the hydraulic conditions in the flume and on the characteristics of the flume return-flow system. For steady-state hydraulic conditions, the form of equation 9 generally assumed to be applicable is

$$
\frac{\partial D}{\partial t}+U \frac{\partial D}{\partial x}=-2.3 k_{2} D
$$

where the DO concentrations have been expressed as deficits. Making the substitutions $x^{\prime}=x-U t$ and $t^{\prime}=t$ into equation 134 , one obtains

$$
\frac{\partial D}{\partial t^{\prime}}=-2.3 k_{2} D
$$

and integrating equation 135 with $D=D_{\mathrm{a}}$ at $t^{\prime}=0$ gives

$$
D=D_{\mathrm{a}} \cdot 10^{-k_{2}} t^{\prime}
$$

or

$$
k_{2}=\frac{1}{t^{\prime}}\left\{\log D_{\mathrm{a}}-\log D\right\}
$$


Equation 137 is identical in form with equation 133; however, in equation 137 , because conditions are not steady state, the flow time $t$ ' is a time lag which must elapse between the DO measurements at the two sampling stations, whereas in equation 133 , because conditions are steady state, the DO measurements at the two stations may be made at any time. In a recirculating flume, it is common experimental practice to obtain an average log deficit difference as compared with the single measurement of the difference obtained in a natural stream or a nonrecirculating flume. The average log deficit is determined by the procedure illustrated in figure 2. The parallel lines show the variation with time of the logarithm of the deficit at two points in the flume a distance $X$ apart. Because the water is recirculated, several points can be obtained on each line, and thus the positions and slopes of the lines can be accurately determined.

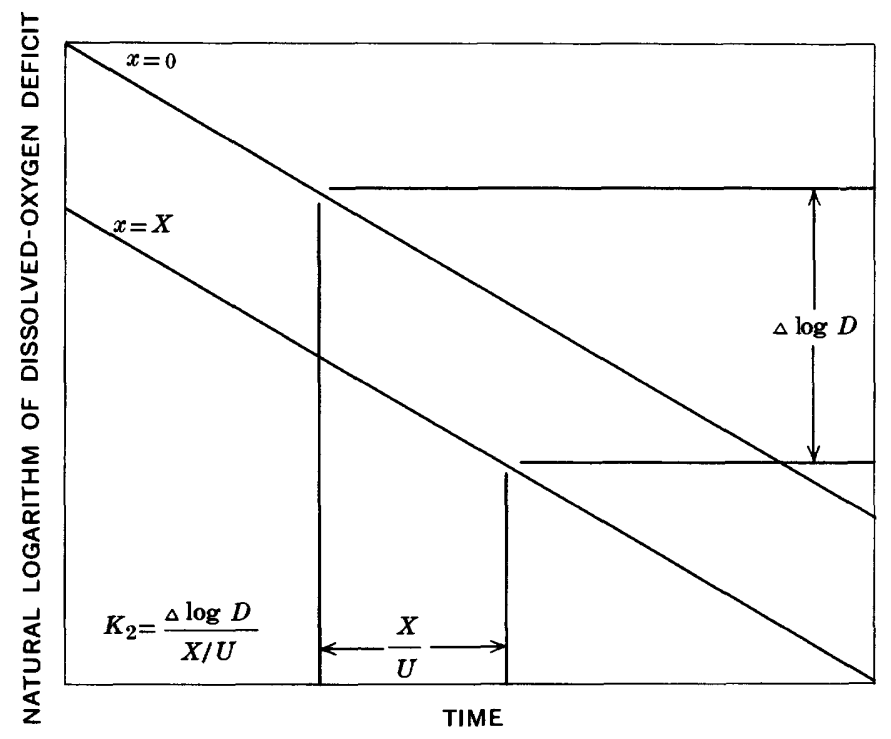

FIGURE 2. - Computation of reaeration coefficient, $K_{2}$, from recirculating-flume data.

\section{DISTURBED-EQUILIBRIUM TECHNIQUE}

The disturbed-equilibrium technique developed by the Water Pollution Research Laboratory (Gameson and Truesdale, 1959) is based on a DO balance described by equation 14 . The procedure consists of measuring the DO concentrations at the upstream and downstream ends of the reach of interest at two different levels of DO concentration. The second DO concentration level is obtained usually by adding sodium sulfite and a cobalt catalyst to the stream, although the diurnal variations in DO concentration caused by photosynthesis may be used. If $P, R, K_{2}, U$, and $C_{s}$ are constant for the reach during the period of the experiment and therefore independent of the DO concentration, then by manipulation of equation 14:

$$
K_{2}=\frac{1}{t} \ln \frac{C_{\mathrm{a}}-C_{\mathrm{a}}^{\prime}}{C_{\mathrm{b}}-C_{\mathrm{b}}{ }^{\prime}}
$$

where $C_{\mathrm{a}}$ and $C_{\mathrm{a}}^{\prime}$ are the upstream DO concentrations at the two levels of concentration, $C_{\mathrm{b}}$ and $C_{\mathrm{b}}$ are the downstream DO concentrations measured at a time $t$ after the upstream concentrations, and $t$ is the flow time for the reach of interest.

Although the respiration rates of bacteria are independent of the DO concentration for concentrations larger than $1 \mathrm{mg} / \mathrm{l}$ (Edwards and others, 1961), the respiration rates of plants (Edwards and others, 1961), and of bottom deposits (McDonnell and Hall, 1969; Edwards and Rolley, 1965) are not. Furthermore, photosynthetic DO production can cause large variations in DO concentration with time on a specific day and from day to day. Therefore, when plants are present, Edwards, Owens, and Gibbs (1961) suggested that the experiments be conducted at night, when the photosynthetic contributions are negligible. To account for the dependence of the plant respiration on DO concentration, they showed that

$$
K_{2}=\frac{1}{H} \frac{Q_{2}-R^{\prime} Q_{1}}{\left(C_{s_{2}}-\bar{C}_{2}\right)-R^{\prime}\left(C_{s_{1}}-\bar{C}_{1}\right)},
$$

where $\bar{C}_{1}$ and $\bar{C}_{2}$ are the average DO concentrations for the study reach for the two levels of DO concentration, $Q_{1}$ and $Q_{2}$ are the average rates of change per unit area of the DO concentration in the reach at each of the two levels of $\mathrm{DO}$ concentration, $C_{s_{2}}$ and $C_{s_{1}}$ are the DO saturation concentrations at the times of the measurements for the DO concentrations at the two levels, and $R^{\prime}$ is the ratio of the oxygen consumed by respiration at DO concentration $\bar{C}_{2}$ to that consumed at DO concentration $\bar{C}_{1}$. The reaeration coefficient obtained from equation 139 is the $K_{2}$ for the average DO deficit in the study reach.

The present authors derived from equation 119 an equation that will give an integral reaeration coefficient rather than one based on the average DO deficit for the reach. If equation 119 is written for upstream deficits of $D_{\mathrm{a}}$ and $D_{\mathrm{a}}^{\prime}$ and the difference taken, the result is

$$
K_{2}=\frac{1}{t} \ln \left[\frac{\left(D_{\mathrm{a}}-D_{\mathrm{a}}{ }^{\prime}\right)+\frac{p-p^{\prime}}{K_{2}}}{\left(D_{\mathrm{b}}-D_{\mathrm{b}}{ }^{\prime}\right)+\frac{p-p^{\prime}}{K_{2}}}\right],
$$

where $p$ and $p^{\prime}$ include the respiration rates of both 
plants and bottom deposits. In equation 119 , the parameter $p$ is the net production of oxygen by photosynthesis. However, the production of DO by photosynthesis is assumed to be constant or zero during the period of the measurement, so that the $p$ parameter becomes strictly a respiration term. The derivation of equation 140 also assumes that $K_{1}, K_{3}, L_{\mathrm{a}}$ and $m$ are constant during the period of the measurements. Because $K_{2}$ appears on both sides of equation 140, a trial-and-error solution is necessary.

The disturbed-equilibrium technique requires measuring the upstream and downstream DO concentrations and the respiration rate in the reach at two different values of the upstream DO deficit. The different values of the upstream deficit may be produced by diurnal variations in photosynthesis and respiration or in BOD inflow, or by the addition of sodium sulfite along with a cobalt catalyst. In general, the technique is very convenient to use, especially if the DO variations are produced by natural conditions in the stream. In this case, however, accurate measurements of the respiration rates $p$ and $p^{\prime}$ are necessary, quantities which are often not easily obtained. If the deficit is produced by sulfite dosing, where $p$ and $p^{\prime}$ are small, there is less uncertainty in the measurements of $p$ and $p^{\prime}$; however, their variation may still be significant. The amount of sulfite required places limitations on the size of stream that can be dosed and on the length of time that the dose can be applied. Furthermore, it must be assured that the sulfite is completely mixed with the flow and that the reaction has proceeded to completion before the flow enters the study reach. Finally, in either method, the experimenter must assure himself that the effect of the time variations in DO and BOD do not cause serious errors in the computation of the reaeration coefficient.

\section{TRACER TECHNIQUE}

Equations 81 and 83 resulting from the kinetictheory model of Tsivoglou (1967) are the basis of the tracer technique for determination of the reaeration coefficient. As discussed previously, Tsivoglou (1967) concluded on the basis of laboratory measurements that the gas-transfer-coefficient ratio in equations 81 and 83 was a constant within the limits of experimental error for the range of temperature and turbulence conditions studied. Because the ratio is constant, an inert gas that does not participate in the various biochemical and biological processes in a stream can be used as a tracer for oxygen. This permits the deter- mination of a true reaeration coefficient in the presence of pollution and photosynthetic effects, provided the value of the ratio of gas-transfer coefficients is known for the inert gas and oxygen. Thus, the tracer technique is unique in that it does not require the measurement of any of the parameters in a DO balance. The technique was first described by Tsivoglou, O'Connell, Walter, Godsil, and Logsdon (1965), a field application was described by Tsivoglou, Cohen, Shearer, and Godsil (1968), and a complete discussion of the technique was presented by Tsivoglou (1967).

The technique uses an instantaneous injection of three tracers at a point upstream from the reach over which the reaeration coefficient is to be measured. The tracers are (1) a fluorescent dye, the purpose of which is to enable field personnel to follow the movement of the tracers; (2) tritiated water, which is used as a conservative dispersion tracer; and (3) the radioactive tracer gas krypton85 , which is used to measure the gas transfer capacity of the flow. Samples of stream water are removed from the flow as the dye peak passes the upstream end and again as it passes the downstream end of the reach. The transfer capacity of the flow for the tracer gas is obtained from the relative concentrations of the gas and tritiated water tracers at the two ends of the reach. Tsivoglou (1967) showed on the basis of an argument involving numbers of krypton and tritium atoms and an exponential expression for gas exchange that

$$
\frac{\left(\frac{C_{G}^{*}}{C_{L}^{*}}\right)_{\mathbf{b}}}{\left(\frac{C_{G}^{*}}{C_{L}^{*}}\right)_{\mathbf{a}}}=e^{-K_{2}{ }^{*} t},
$$

where $C^{*}$ is concentration; $t$ is flow time, or $\Delta x / U$, where $\Delta x$ is the distance between the downstream and upstream stations indicated respectively by the subscripts "b" and "a"; the term $K_{2_{G}}$ is the gastransfer coefficient of the tracer gas; and the $G$ and $L$ subscripts refer respectively to the krypton and tritium tracers.

The tracer-gas transfer coefficient, $K_{{ }_{2}}$, can be converted to a reaeration coefficient, $K_{2}$, by means of the gas-transfer ratio determined by Tsivoglou (1967). He found that the average ratio of the rate of krypton desorption to the rate of oxygen absorption was 0.83 . Hence,

$$
K_{2}=\frac{K_{2_{G}}}{0.83}
$$

The crucial assumption in the tracer technique is that this ratio is a constant-that is, independent of the flow conditions, temperature, and pollutants present. 
We developed a theoretical basis for the technique from solutions of the conservation-of-mass equation for the two tracers. The one-dimensional diffusion equation in a steady-uniform flow for a conservative tracer is

$$
\frac{\partial C_{L}^{*}}{\partial t}+U \frac{\partial C_{L}^{*}}{\partial x}=D_{x} \frac{\partial^{2} C_{L}^{*}}{\partial x^{2}},
$$

where $C_{L}^{*}$ is the tracer concentration. The corresponding equation for the gaseous tracer is

$$
\frac{\partial C_{G}^{*}}{\partial t}+U \frac{\partial C_{G}^{*}}{\partial x}=D_{x} \frac{\partial^{2} C_{G}^{*}}{\partial x^{2}}-K_{2_{G}} C_{G}^{*}
$$

where $C_{G}^{*}$ is the tracer gas concentration. Implicit in the use of the one-dimensional diffusion equation is the assumption that the tracers are uniformly distributed in the cross section of the stream. Therefore, the upstream sampling point must be far enough downstream from the injection point so that complete mixing in both the lateral and vertical directions has been achieved.

The solutions to equations 143 and 144 for instantaneous inputs of weight $W_{L}$ of the liquid and $W_{G}$ of the gaseous tracer at $x=0$ and $t=0$ were presented by Camp (1963). For equation 143, the solution is

$$
C_{L}^{*}=\frac{W_{0_{L}}}{2 \sqrt{\pi D_{x} t}} e^{-\frac{(x-U t)^{2}}{4 D_{x} t}},
$$

and for equation 144 ,

$$
C_{G}^{*}=\frac{W_{0_{G}}}{2 \sqrt{\pi D_{x} t}} e^{-\left[\frac{(x-v t)^{2}}{4 D_{x} t}+K_{2} G_{G} t\right]},
$$

in which $W_{0_{L}}=W_{L} / A_{x}$ and $W_{0_{G}}=W_{G} / A_{x}$, where $A_{x}$ is the flow area. For any longitudinal position $x$ at time $t$, the ratio of the gas concentration to the liquid concentration is

$$
\frac{C_{G}^{*}}{C_{L}^{*}}=\frac{W_{0_{G}}}{W_{0_{L}}} e^{-K_{2} G^{t}} .
$$

If one now takes the ratio of equation 147 for the downstream station at a time $t=\Delta x / U$ to equation 147 for the upstream station at $t=0$, the result is identical with equation 141 given previously by Tsivoglou (1967). Using equations 141 and 142 and the four measured tracer concentrations, the reaeration coefficient $K_{2}$ can be computed.

The arguments used by Tsivoglou (1967) in developing equation 141 require no assumptions regarding vertical and lateral mixing of the tracers. However, the reaeration coefficient obtained from the tracer technique using a pointsource injection and samples from the centerline of the channel is representative only of the filament of the stream traversed by the tracers in moving from the injection point to the sampling point. To obtain a $K_{2}$ representative of the entire cross section of a reach, the tracers must be injected sufficiently far upstream so that they will be uniformly distributed in the cross section before entering the reach. Under these conditions, the one-dimensional diffusion equation is applicable, and as shown previously, a theoretical basis exists for the tracer-technique equation obtained from intuitive arguments by Tsivoglou (1967).

The assumptions on which the tracer technique is based were listed by Tsivoglou, Cohen, Shearer, and Godsil (1968, p. 302) as follows:

(1) The tritiated water undergoes dispersion only in the stream, and is not lost from the stream water in any significant amount; (2) the dissolved krypton-85 undergoes the same dispersion as the tritiated water, in addition is lost to the atmosphere, and is not otherwise lost in any significant amount; (3) the ratio (0.83) of gas transfer coefficients for dissolved krypton-85 and oxygen is not affected significantly by temperature, turbulence, or the presence of usual water pollutants, in the range of interest***.

The tritium and krypton tracers were chosen with the first two assumptions in mind, and therefore these assumptions should be valid. The third assumption was verified experimentally, as discussed previously.

The tracer technique has several advantages over the DO balance and disturbed-equilibrium techniques for determination of reaeration coefficients. The most obvious advantage is that only the concentrations of two radioactive substances need be determined as compared with all the various parameters that must be measured in the other two techniques. In addition, the nuclear counting techniques are very accurate, and because only ratios of the counts are used, determination of the counting efficiency is not necessary, provided it is the same for the counting of all samples. The possibility exists also of determining with the tracers the discharge and longitudinaldispersion characteristics of the stream at the same time that the reaeration coefficient is measured. This would require additional effort, however, because the complete concentrationversus-time curve at each cross section is necessary whereas only the peak concentrations are needed for the reaeration-coefficient determination.

There are also disadvantages to the tracer technique. Radiation exposure received by the personnel conducting the study and the public along the stream may be significant. Tsivoglou (1967) reported that radiation exposure could be eliminated with a minimum of trouble for discharges less than $200 \mathrm{cfs}$. However, for larger flows, more stringent precautions are required because of the 
larger amounts of tracer necessary. Another disadvantage of using radioactive tracers is that the process for obtaining a license to use radioactive material in a public waterway is quite involved. Finally, the nuclear counting equipment necessary for tritium and krypton is expensive and not field-type equipment; hence, samples must be returned to a laboratory for analysis.

\section{ANALYSIS OF ERRORS IN REAERATION-COEFFICIENT CALCULATION TECHNIQUES}

The determination of the reaeration coefficient by any of the techniques described previously is subject to many sources of errors. The major sources of error are (1) differences between the dynamic stream condition and the quiescent condition in the various bottle techniques developed for measuring the DO balance parameters, (2) the difficulty and expense of obtaining a sufficient number of representative samples to describe accurately the stream conditions at the cross section of interest, (3) deviations from the assumptions inherent in the derivation of the $\mathrm{DO}$ balance equation, and (4) the accuracy of the DO measurement procedure used in the various techniques for determining the DO balance parameters.

A general statistical analysis of the effect of these various sources of error on the calculation of the reaeration coefficient is beyond the scope of this report and probably is not feasible with the present state of our knowledge. However, it is instructive and important to consider the range of possible errors in the measured DO balance parameters and the effect of these errors on the DO balance equation used to calculate the reaeration coefficient.

The purpose of this section is to present a discussion of (1) the sensitivity of the DO balance technique to errors in the measured parameters; (2) the sensitivity of the disturbed-equilibrium technique to errors in the measured parameters; (3) possible errors in the tracer technique; and (4) reproducibility of reaeration coefficients measured in flumes.

ACCURACY OF MEASURED DISSOLVED-OXYGEN BALANCE PARAMETERS

The accuracy of the measured value of a DO balance parameter is dependent not only on the accuracy of the DO measurements but also on the extent to which the measurement conditions under which the measurements are made simulate the conditions in the flow, and on how representative the samples are of the total flow.
For example, as has already been mentioned, not only is accurate measurement of BOD in bottles difficult due to dilution and seeding problems, but also there is considerable question as to how accurately the behavior of a sample of water incubated under quiescent conditions in a constant temperature bath simulates the behavior of a similar slug of water under dynamic streamflow conditions and varying temperature. There are similar problems with the techniques for determining the rate of oxygen consumption by the benthos and for determining the rates of photosynthesis and respiration of suspended and attached plants.

Completely aside from the problem of simulating field conditions with measurement techniques is the problem of obtaining representative samples in the field. The uniformity of DO and BOD distribution in a cross section will depend on a multiplicity of factors, among which the most important are distance from sewage outfalls and stream tributaries, shape of cross section, tortuosity of channel, and bed roughness size, shape, and spacing. For example, Churchill, Elmore, and Buckingham (1962, figs. 3-9) found a consistent U-shaped DO distribution across the stream channel and attributed this distribution to (1) incomplete mixing into the main stream of flow from upstream tributaries, (2) increased reaeration near the banks due to a longer traveltime, and (3) unequal effects across the stream of photosynthesis and respiration by attached aquatics. Considering the large cross-sectional DO variation and the small longitudinal variation in average DO illustrated in these figures, one would expect considerable error in the calculated $k_{2}$ if DO were sampled at only one point in the cross section. Another example of the difficulty of obtaining representative samples for the computation of the DO balance parameters was given by Owens and Edwards (1961), who took extensive samples of attached aquatic plants from several English streams for analysis of the photosynthesis-respiration parameter. In their studies, the standard deviation of the amount of the attached aquatics per unit area averaged 32 percent of the mean. The size of this standard deviation in terms of the mean indicates the difficulty of obtaining a reliable estimate of photosynthesis or respiration without sampling the plants on a large area of the stream bed.

Because of the difficulty of reproducing the stream environment in the measurement techniques for DO balance parameters, and because of the difficulty and expense of obtaining representa- 
tive samples, it is apparent that if all parameters in the DO balance equation are significant, then the two conditions discussed above, and not DO measurements themselves, limit the accuracy which can be obtained in the calculation of $k_{2}$. Because of the present state of uncertainty about these two situations, the accuracy obtainable from DO balance calculations is largely a matter of conjecture. The error analysis techniques used in this section have been designed with this in mind. Reasonable average values for the various DO balance parameters and what the authors consider to be reasonable values of errors are given in this section.

\section{DISSOLVED-OXYGEN BALANCE TECHNIQUE}

Because of the complexity of the general DO balance equation, the number of parameters involved, and the difficulty of reproducing field conditions for replications of $k_{2}$ determinations, a rigorous statistical analysis of the effect of errors on the calculated $k_{2}$ cannot be performed at the present time. Alternatively, however, one can perform for $k_{2}$ a sensitivity analysis, similar to that of Kothandaraman and Ewing (1969), on the downstream DO deficit calculated from the DO balance equation. The sensitivity analysis is designed to yield the error in the calculated value of $k_{2}$, when the error in another parameter of the DO balance equation is known. When the distribution functions of the errors in the parameters of the DO balance equation are known, a Monte Carlo approach similar to that used by Kothandaraman and Ewing (1969) can be used to determine the distribution function of the calculated $k_{2}$ values.

A sensitivity analysis consists of observing the behavior of the output of a system with all except one of its parameters constant. This parameter is varied throughout the range of possible values, and at any given value of the parameter, the sensitivity of the system to the parameter is the change in output per unit change in the parameter. The sensitivity analysis was performed on the general DO balance equation, equation 119 , written in the form

$$
\begin{aligned}
f\left(k_{2}\right) & =\frac{k_{1}}{k_{2}-\left(k_{1}+k_{3}\right)}\left[L_{\mathrm{a}}-\frac{m}{2.3\left(k_{1}+k_{3}\right)}\right]\left[10^{\left.-\left(k_{1}+k_{3}\right) t-10^{-k_{2} t}\right]}\right. \\
& +\frac{k_{1}}{k_{2}}\left[\frac{m}{2.3\left(k_{1}+k_{3}\right)}-\frac{p}{2.3 k_{1}}\right]\left[1-10^{-k_{2} t}\right]+D_{\mathrm{a}} 10^{-k_{2} t}-D_{\mathrm{b}} .
\end{aligned}
$$

Equation 148 was solved for $k_{2}$ using the NewtonRaphson iterative method (Kunz, 1957) in the form

$$
\left(k_{2}\right)_{i+1}=\left(k_{2}\right)_{i}-\frac{f\left[\left(k_{2}\right)_{i}\right]}{f^{\prime}\left[\left(k_{2}\right)_{i}\right]}
$$

and calculations were stopped when the absolute value of the ratio on the right-hand side of equation 149 was less than 0.001 times the true value of $k_{2}$.

The sensitivity analysis consisted of the following steps:

1. Assume a typical set of parameters for use in the DO balance equation, equation 119. This set of parameters is called a BPS (basic parameter set).

2. Substitute the BPS into equation 119, and calculate the corresponding BPS value of $D_{\mathrm{b}}$.

3 . Use equations 148 and 149 to compute the sensitivity of equation 119 to each of the parameters in turn.

In some calculations, the classical sensitivity analysis procedure was changed in that several of the parameters were varied simultaneously.

The BPS values are given in table 1 . Typical sensitivity analysis plots are given in figures 3

\begin{tabular}{|c|c|c|c|c|c|c|c|c|c|}
\hline $\begin{array}{l}\text { BPS } \\
\text { No. }\end{array}$ & $\begin{array}{c}k_{1} \\
\left(\text { days }^{-1}\right)\end{array}$ & $\begin{array}{c}k_{2} \\
\left(\text { days }^{-1}\right)\end{array}$ & $\begin{array}{c}k_{3} \\
\left(\text { days }^{-1}\right)\end{array}$ & $\underset{\text { perday) }}{p}$ & $\underset{\substack{m \\
\text { per day } \\
m}}{ }$ & $\underset{(\mathrm{mg} / \mathrm{l})}{D_{\mathrm{a}}}$ & $\underset{(\mathrm{mg} / \mathrm{l})}{L_{\mathrm{a}}}$ & $\stackrel{t}{\text { (days) }}$ & $\underset{(\mathrm{mg} / \mathrm{l})}{D_{\mathrm{b}}}$ \\
\hline $\begin{array}{l}1 \\
2 \\
3 \\
4 \\
5\end{array}$ & $\begin{array}{r}0.1 \\
.1 \\
.1 \\
.1 \\
.1\end{array}$ & $\begin{array}{l}1 \\
1 \\
1 \\
1 \\
1\end{array}$ & $\begin{array}{l}0 \\
0 \\
.1 \\
0 \\
0\end{array}$ & $\begin{array}{l}0 \\
0 \\
0 \\
1 \\
0\end{array}$ & $\begin{array}{l}0 \\
0 \\
0 \\
0 \\
0\end{array}$ & $\begin{array}{l}\mathbf{3} \\
\mathbf{3} \\
\mathbf{3} \\
\mathbf{3} \\
\mathbf{3}\end{array}$ & $\begin{array}{r}5 \\
0 \\
5 \\
5 \\
200\end{array}$ & $\begin{array}{r}0.05 \\
.05 \\
.05 \\
.05 \\
.05\end{array}$ & $\begin{array}{l}2.73 \\
2.67 \\
2.73 \\
2.68 \\
4.84\end{array}$ \\
\hline $\begin{array}{r}6 \\
7 \\
8 \\
9 \\
10\end{array}$ & $\begin{array}{l}.1 \\
.1 \\
.1 \\
.1 \\
.1\end{array}$ & $\begin{array}{c}10^{.2} \\
10.2 \\
1^{2}\end{array}$ & $\begin{array}{l}0 \\
0 \\
0 \\
0 \\
0\end{array}$ & $\begin{array}{l}0 \\
0 \\
0 \\
0 \\
0\end{array}$ & $\begin{array}{l}0 \\
0 \\
0 \\
0 \\
0\end{array}$ & $\begin{array}{l}\mathbf{3} \\
\mathbf{3} \\
\mathbf{3} \\
\mathbf{3} \\
\mathbf{3}\end{array}$ & $\begin{array}{r}5 \\
5 \\
200 \\
200 \\
200\end{array}$ & $\begin{array}{l}.05 \\
.05 \\
.05 \\
.05 \\
.05\end{array}$ & $\begin{array}{r}2.99 \\
.98 \\
2.31 \\
5.20 \\
4.84\end{array}$ \\
\hline $\begin{array}{l}11 \\
12 \\
13 \\
14 \\
15\end{array}$ & $\begin{array}{l}.1 \\
.4 \\
.4 \\
.1 \\
.1\end{array}$ & $\begin{array}{l}1 \\
1 \\
1 \\
1 \\
1\end{array}$ & $\begin{array}{l}0 \\
0 \\
0 \\
0 \\
0\end{array}$ & $\begin{array}{l}0 \\
0 \\
0 \\
0 \\
0\end{array}$ & $\begin{array}{l}0 \\
0 \\
0 \\
0 \\
0\end{array}$ & $\begin{array}{l}\mathbf{5} \\
\mathbf{5} \\
\mathbf{5} \\
\mathbf{3} \\
\mathbf{3}\end{array}$ & $\begin{array}{r}5 \\
5 \\
50 \\
5 \\
50\end{array}$ & $\begin{array}{l}.05 \\
.05 \\
.05 \\
.05 \\
.05\end{array}$ & $\begin{array}{l}4.51 \\
4.67 \\
6.58 \\
2.73 \\
3.21\end{array}$ \\
\hline $\begin{array}{l}16 \\
17 \\
18 \\
19\end{array}$ & $\begin{array}{l}.1 \\
.1 \\
.1 \\
.1\end{array}$ & $\begin{array}{l}1 \\
1 \\
1 \\
1\end{array}$ & $\begin{array}{l}0 \\
0 \\
0 \\
0\end{array}$ & $\begin{array}{r}20 \\
20 \\
10 \\
5\end{array}$ & $\begin{array}{l}0 \\
0 \\
0 \\
0\end{array}$ & $\begin{array}{l}\mathbf{3} \\
\mathbf{3} \\
\mathbf{3} \\
\mathbf{3}\end{array}$ & $\begin{array}{r}5 \\
200 \\
5 \\
5\end{array}$ & $\begin{array}{l}.05 \\
.05 \\
.05 \\
.05\end{array}$ & $\begin{array}{l}1.78 \\
3.89 \\
2.26 \\
2.49\end{array}$ \\
\hline
\end{tabular}

TABLE 1. - Basic parameter sets used in the sensitivity analyses of the DO balance equation

through 8. The ordinates in these figures are expressed as a percentage of the BPS value of $k_{2}$, and the abscissas are in the dimensions of the parameter that is being varied in the sensitivity analyses. Of the 19 BPS considered in the sensitivity analyses, six, in general, were selected for presentation in figures 3 through 8 . Numbers 1,5 , $6,7,15$, and 16 were selected as being typical of the following field conditions: 1 , A medium-sized unpolluted river; 5, a medium-sized heavily polluted river; 6, a large unpolluted river; 7 , a shallow, swift-flowing stream; 15 , a medium-size moderately polluted river; and 16 , a medium-sized unpolluted river in which the effect of ignoring a photosynthetic-oxygen production rate of 20 $\mathrm{mg} / \mathrm{l}$ per day is investigated. 


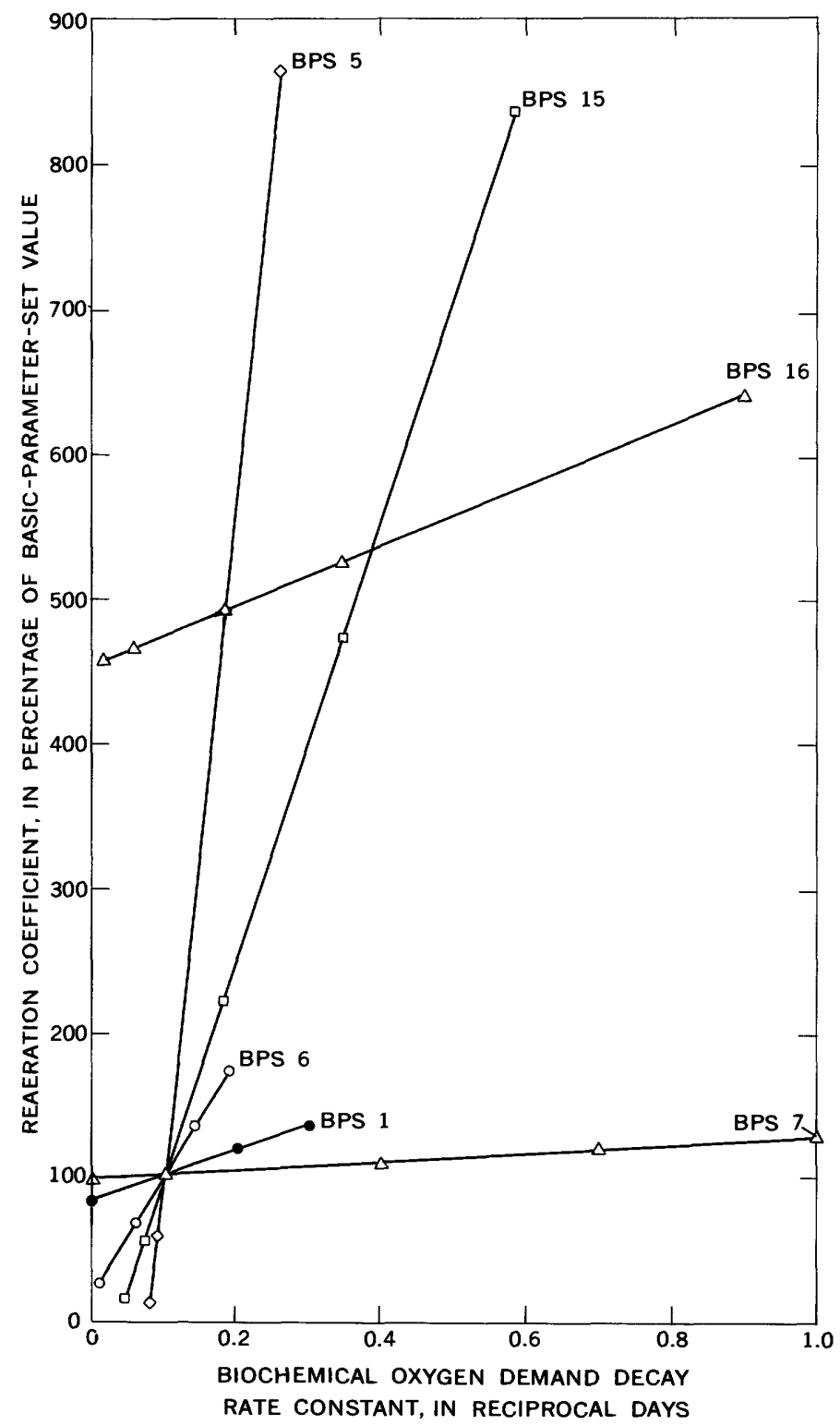

FIGURE 3. - Sensitivity of reaeration coefficient to rate constant for BOD decay, dissolved-oxygen balance technique.

The sedimentation rate constant, $k_{3}$, and $m$, the rate of addition of BOD from bottom deposits, were not considered in figures 3 through 8 because the sensitivity analyses showed that the dependence of $k_{2}$ on these parameters was very small. For $k_{3}$, the most sensitive example was for BPS 5, where $k_{2}$ changed only 23 percent of its BPS value for a change of $k_{3}$ from 0 to 0.8 . For $m$, the most sensitive example was for BPS 10 , where $k_{2}$ changed only 13 percent as $m$ varied from 0 to $200 \mathrm{mg} / \mathrm{l}$ per day.

The results shown in figures 3 through 8 can be clarified using an approximate form of equation 119. Because the exponents in this equation are much less than 1 , the exponential terms may be

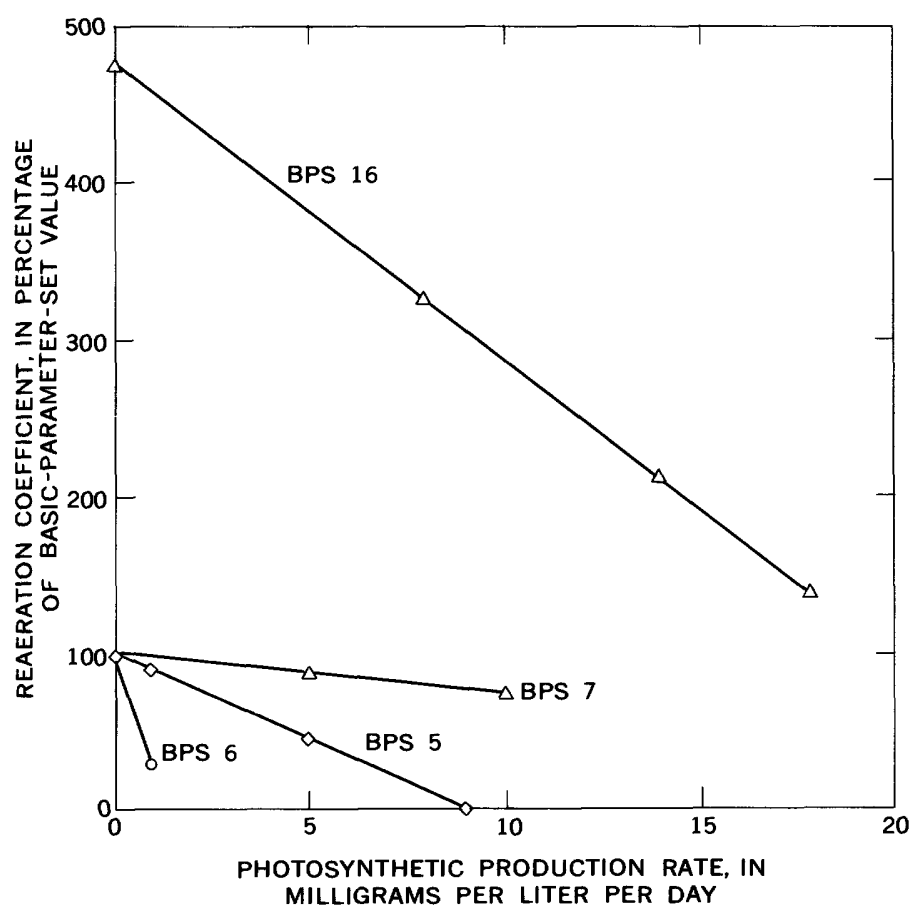

FIGURE 4. - Sensitivity of reaeration coefficient to photosynthetic-oxygen production rate, dissolved-oxygen balance technique.

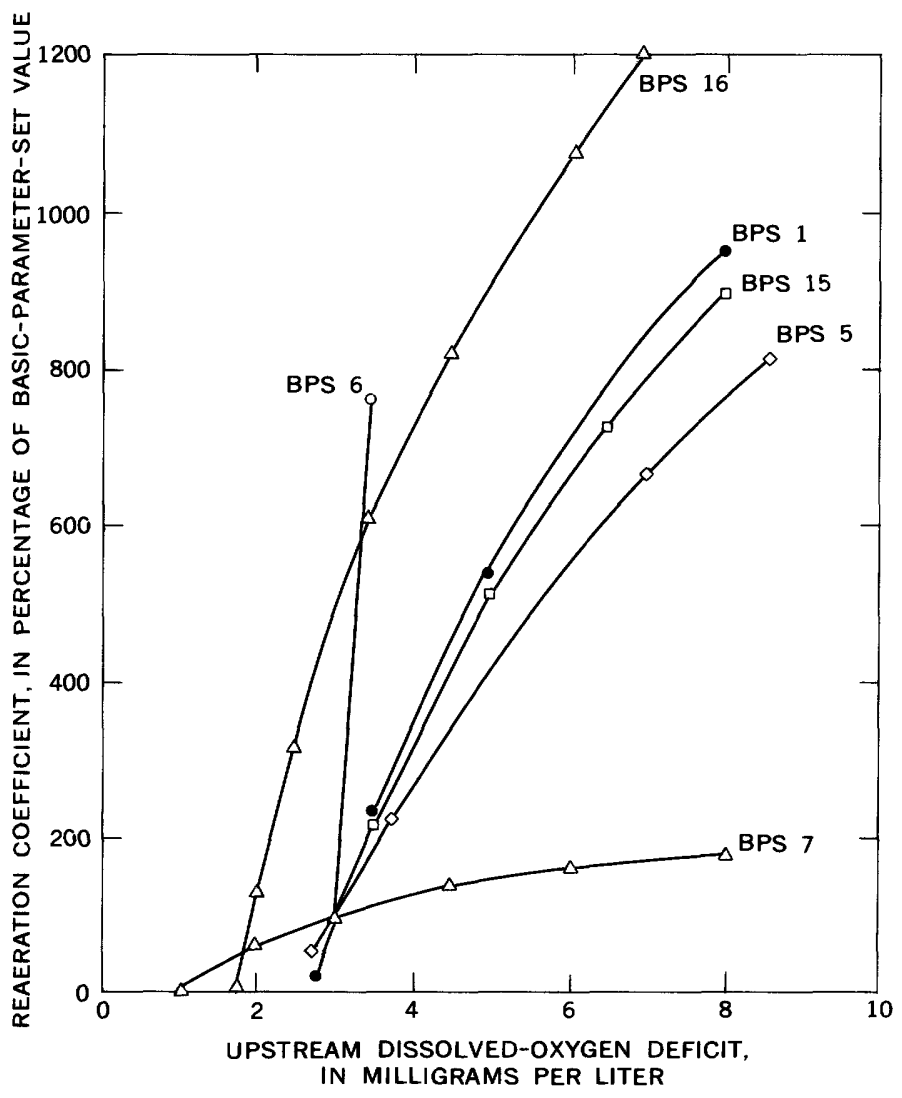

FiguRE 5. - Sensitivity of reaeration coefficient to upstream DO deficit, dissolved-oxygen balance technique. 


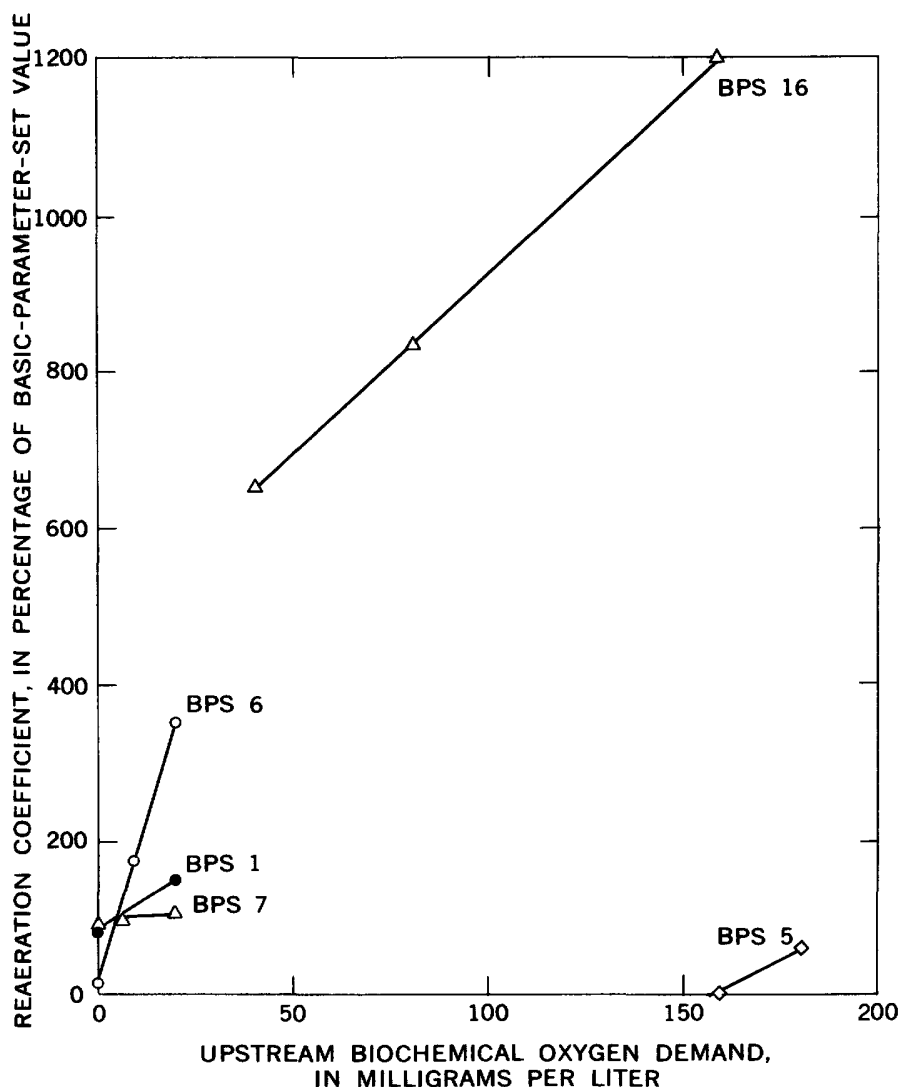

FIgURE 6. - Sensitivity of reaeration coefficient to upstream BOD, dissolved-oxygen balance technique.

approximated by the first two terms in their Taylor-series expansions. In this case, equation 119 reduces to

$$
k_{2} \cong \frac{1}{2.3}\left[\frac{1}{t}\left(1-\frac{D_{\mathrm{b}}}{D_{\mathrm{a}}}\right)+\frac{2.3 k_{1} L_{\mathrm{a}}}{D_{\mathrm{a}}}-\frac{p}{D_{\mathrm{a}}}\right] .
$$

The first term in the brackets of equation 150 explains why the sensitivity of $k_{2}$ to variations in flow time, $t$, decreases as $D_{\mathrm{b}}$ approaches $D_{\mathrm{a}}$ (fig. 7). The second and third terms in the brackets explain the linear variation of $k_{2}$ in terms of $k_{1}, p$, and $L_{\mathrm{a}}$ (figs. 3,4 , and 6). The second term in the brackets explains the changes in sensitivity of $k_{2}$ to $k_{1}$ with changes in $L_{\mathrm{a}}$. Finally, the absence of $k_{3}$ and $m$ from equation 150 explains the lack of sensitivity of the calculated $k_{2}$ to these parameters. The solution of equation 150 for $k_{2}$ using BPS 1 is 0.94 , and that using BPS 5 is 1.37 ; thus, the approximations in the equation are too great for it to be used for calculation of $k_{2}$ directly, but it is valuable for showing quickly the relative seriousness of the errors in the calculated $k_{2}$ which can result from errors in the other parameters.

The extension of the sensitivity analysis to the determination of the error in a reaeration-

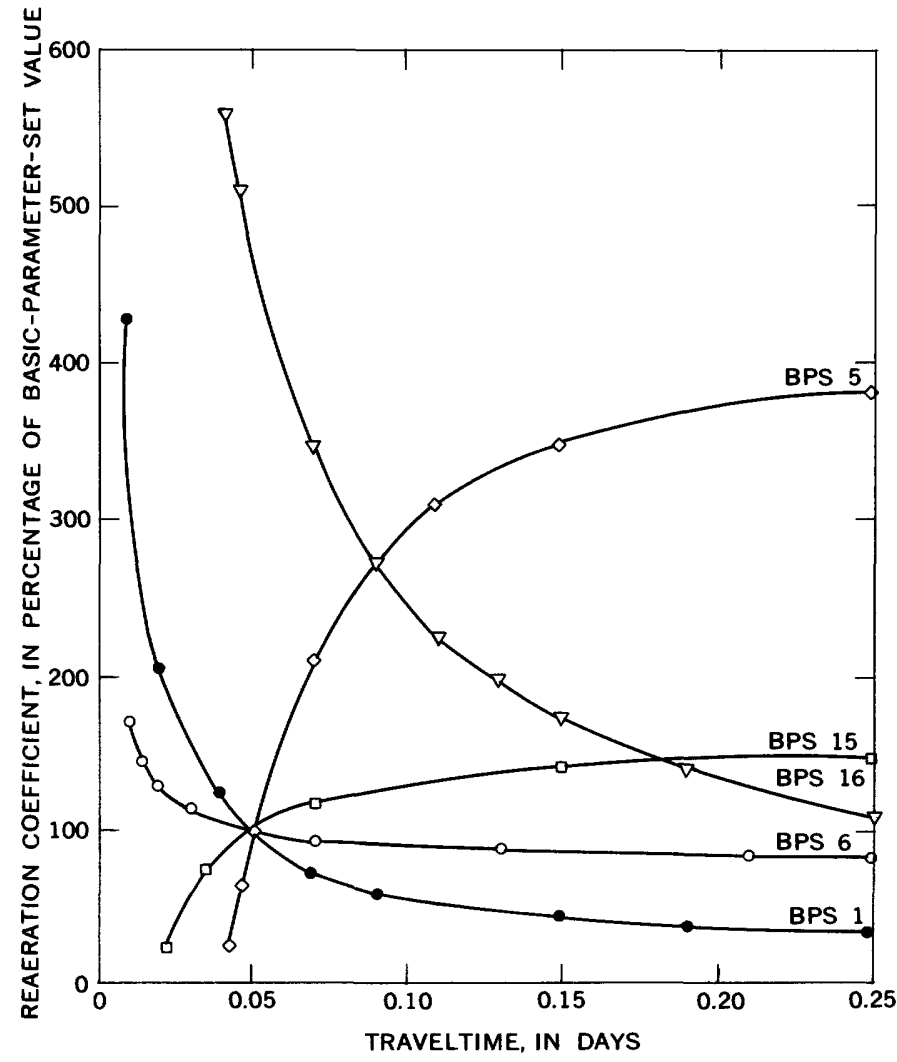

FIgURE 7. - Sensitivity of reaeration coefficient to traveltime, dissolved-oxygen balance technique.

coefficient calculation resulting from an error in a measured parameter requires information on the magnitudes of the errors to be expected in the measured parameters. However, as discussed previously, the different techniques developed for measuring the DO balance parameters are subject to various types of errors, and the determination of such errors is difficult. Therefore, for the purpose of an error analysis we have made what we consider to be reasonable assumptions of the RMS (root-mean-square) and maximum errors to be expected in the various measured parameters. These estimates are presented in table 2. The RMS error is the square root of the mean value of the square of the deviations of the measured parameter from the true value of the parameter.

The errors in a calculated $k_{2}$ corresponding to the estimated parameter errors given in table 2 are given by the product of the estimated error and the slope of the sensitivity-analysis plot. It is apparent from figures 3 through 8 that the slopes of the sensitivity-analysis plots depend on the particular BPS considered. Therefore, the minimum, average, and maximum slopes were deter- 


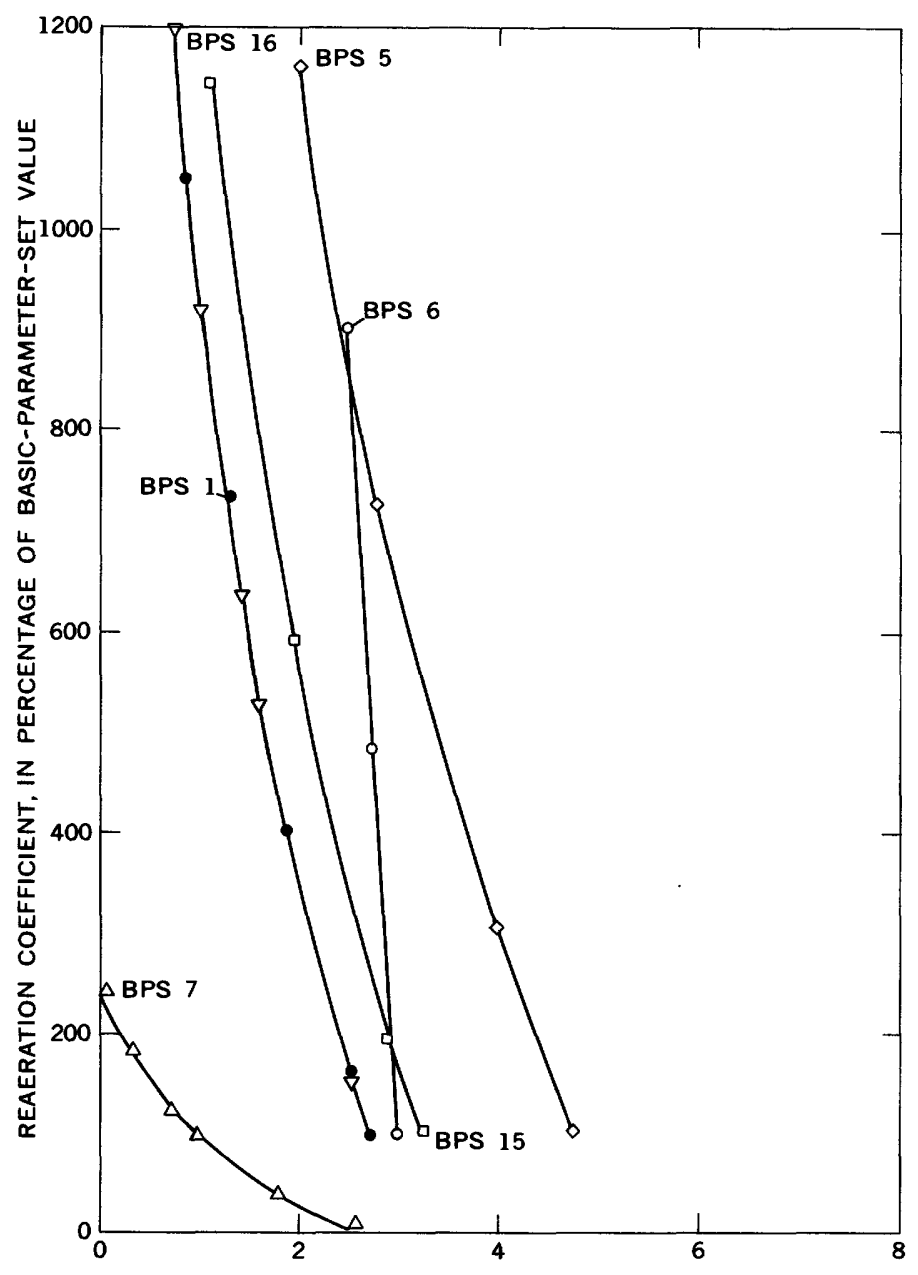

DOWNSTREAM DISSOLVED-OXYGEN DEFICIT, IN MILLIGRAMS PER LITER

FIGURE 8. - Sensitivity of reaeration coefficient to downstream DO deficit, dissolved-oxygen balance technique.

TABLE 2. - Assumed errors in measurements of DO balance parameters

\begin{tabular}{|c|c|c|c|c|}
\hline \multirow{2}{*}{ Parameter } & \multicolumn{4}{|c|}{ Assumed error } \\
\hline & \multicolumn{2}{|c|}{ Root-mean-square } & \multicolumn{2}{|r|}{ Maximum } \\
\hline$k_{1}$ & 0.05 & $\operatorname{days}^{-1}$ & 0.30 & $\operatorname{days}^{-1}$ \\
\hline$k_{3}$ & .10 & days $^{-1}$ & .50 & $\operatorname{days}^{-1}$ \\
\hline$D_{\mathrm{a}}$ & .10 & $\mathrm{mg} / 1$ & 1.0 & $\mathrm{mg} / 1$ \\
\hline$D_{\mathrm{b}}$ & .10 & $\mathrm{mg} / \mathrm{l}$ & 1.0 & $\mathrm{mg} / \mathrm{l}$ \\
\hline$L_{\mathrm{a}}$ & 2.0 & $\mathrm{mg} / \mathrm{l}$ & 10 & $\mathrm{mg} / \mathrm{l}$ \\
\hline$p$ & & $\mathrm{mg} / 1$ per day & 10 & $\mathrm{mg} / 1$ per day \\
\hline$m$ & 5.0 & $\mathrm{mg} / \mathrm{l}$ per day & 20 & mg/l per day \\
\hline$t$ & .002 & day & .005 & day \\
\hline
\end{tabular}

mined for each parameter so that the entire range of conditions covered by the 19 different BPS would be considered. The slopes are presented in table 3 . The minimum, average, and maximum slopes were combined, each in turn, with the RMS and maximum error estimates from table 2 to give the expected errors in the calculated reaera- tion coefficient. These errors are presented in table 4. It is apparent that, with the exception of $m$, errors in all the parameters may cause considerable error in the calculated $k_{2}$ value. As the value of $k_{2}$ decreases, the percentage of error increases.

TABLE 3. - Slopes of the sensitivity-analysis plots

\begin{tabular}{|c|c|c|c|}
\hline \multirow{2}{*}{ Parameter } & \multicolumn{3}{|c|}{ Slope } \\
\hline & Minimum & Average & Maximum \\
\hline $\begin{array}{c}k_{1} \\
k_{3} \\
D_{\mathrm{a}} \\
D_{\mathrm{b}} \\
L_{\mathrm{a}} \\
p \\
m \\
t\end{array}$ & $\begin{array}{c}1.6 \\
0.01 \\
1.4(\mathrm{mg} / 1 \text { per day })^{-1} \\
1.6(\mathrm{mg} / \mathrm{l} \mathrm{per} \mathrm{day})^{-1} \\
0.02(\mathrm{mg} / \mathrm{l} \mathrm{per} \mathrm{day})^{-1} \\
0.11(\mathrm{mg} / 1)^{-1} \\
1 \times 10^{-1 /\left(\mathrm{mg} / \mathrm{l}^{-1}\right.} \\
5.0 \text { days }^{-2}\end{array}$ & $\begin{array}{cc} & 10 \\
& 0.05 \\
2.0 & (\mathrm{mg} / \mathrm{l} \text { per day })^{-1} \\
2.5 & (\mathrm{mg} / \mathrm{l} \text { per day })^{-1} \\
0.035(\mathrm{mg} / \mathrm{l} \text { per day })^{-1} & 0.20(\mathrm{mg} / \mathrm{l})^{-1} \\
5 \times 10^{-4}(\mathrm{mg} / \mathrm{l})^{-1} \\
\mathbf{4 0} \text { days }^{-2}\end{array}$ & $\begin{array}{c}80 \\
0.50 \\
3.0(\mathrm{mg} / \mathrm{l} \text { per day })^{-1} \\
6.9(\mathrm{mg} / \mathrm{l} \text { per day })^{-1} \\
0.08(\mathrm{mg} / \mathrm{l} \text { per day })^{-1} \\
0.30\left(\mathrm{mg} / \mathrm{l}^{-1}\right. \\
2 \times 10^{-3}(\mathrm{mg} / \mathrm{l})^{-1} \\
150 \mathrm{days}^{-2}\end{array}$ \\
\hline
\end{tabular}

TABLE 4.-Errors in the calculated reaeration coefficient resulting from errors in parameter measurement, $D O$ balance technique

\begin{tabular}{ccccccc}
\hline & \multicolumn{6}{c}{ Error in calculated $k_{2}$, in days day $^{-1}$} \\
\cline { 2 - 8 } Parameter & \multicolumn{2}{c}{ Minimum slope } & \multicolumn{2}{c}{ Average slope } & \multicolumn{2}{c}{ Maximum slope } \\
\cline { 2 - 8 } & $\begin{array}{c}\text { RMS } \\
\text { error }\end{array}$ & $\begin{array}{c}\text { Maximum } \\
\text { error }\end{array}$ & $\begin{array}{c}\text { RMS } \\
\text { error }\end{array}$ & $\begin{array}{c}\text { Maximum } \\
\text { error }\end{array}$ & $\begin{array}{c}\text { RMS } \\
\text { error }\end{array}$ & $\begin{array}{c}\text { Maximum } \\
\text { error }\end{array}$ \\
\hline$k_{\mathrm{t}}$ & 0.08 & 0.48 & 0.50 & 3.0 & 4.0 & 24 \\
$k_{3}$ & .001 & .005 & .005 & .025 & .05 & .25 \\
$D_{\mathrm{a}}$ & .14 & 1.4 & .20 & 2.0 & .30 & 3.0 \\
$D_{\mathrm{b}}$ & .16 & 1.6 & .25 & 2.5 & .69 & 6.9 \\
$L_{\mathrm{a}}$ & .04 &. .20 & .07 & .35 & .16 & .80 \\
$p$ & .22 & 1.1 & .40 & 2.0 & .60 & 3.0 \\
$m$ & $5 \times 10^{-4}$ & $2 \times 10^{-3}$ & $2.5 \times 10^{-3}$ & .01 & .01 & .04 \\
$t$ & .01 & .025 & .08 & .20 & .30 & .75 \\
\hline
\end{tabular}

Figures 5 and 8 show the sensitivity of the calculated $k_{2}$ to the upstream and downstream DO deficits, respectively. Because the lines slope in opposite directions, the effect of systematic errors in the procedure used to determine the DO should tend to cancel. This was verified with calculations based on BPS 14. The reaeration coefficient was calculated from equations 148 and 149 using a systematic error in the upstream and downstream DO deficits, and the results are presented in figure 9. The abscissa is in terms of the systematic error in DO and is expressed as a percentage of the upstream deficit or a percentage of the deficit range. It is apparent from comparison of figures 9 and 5 or figures 9 and 8 that the effect of the systematic error is much less severe than the effect of a random error of the same magnitude in one of the DO deficits. The use of an incorrect value of the saturation concentration, $C_{s}$, in the computation of the DO deficits will have an effect identical with a systematic error in the DO measurement procedure.

The effect on the calculated $k_{2}$ of ignoring photosynthetic production is shown in figure 10 . The downstream DO deficits for BPS 16,18 , and 19 were calculated with the photosynthetic production 


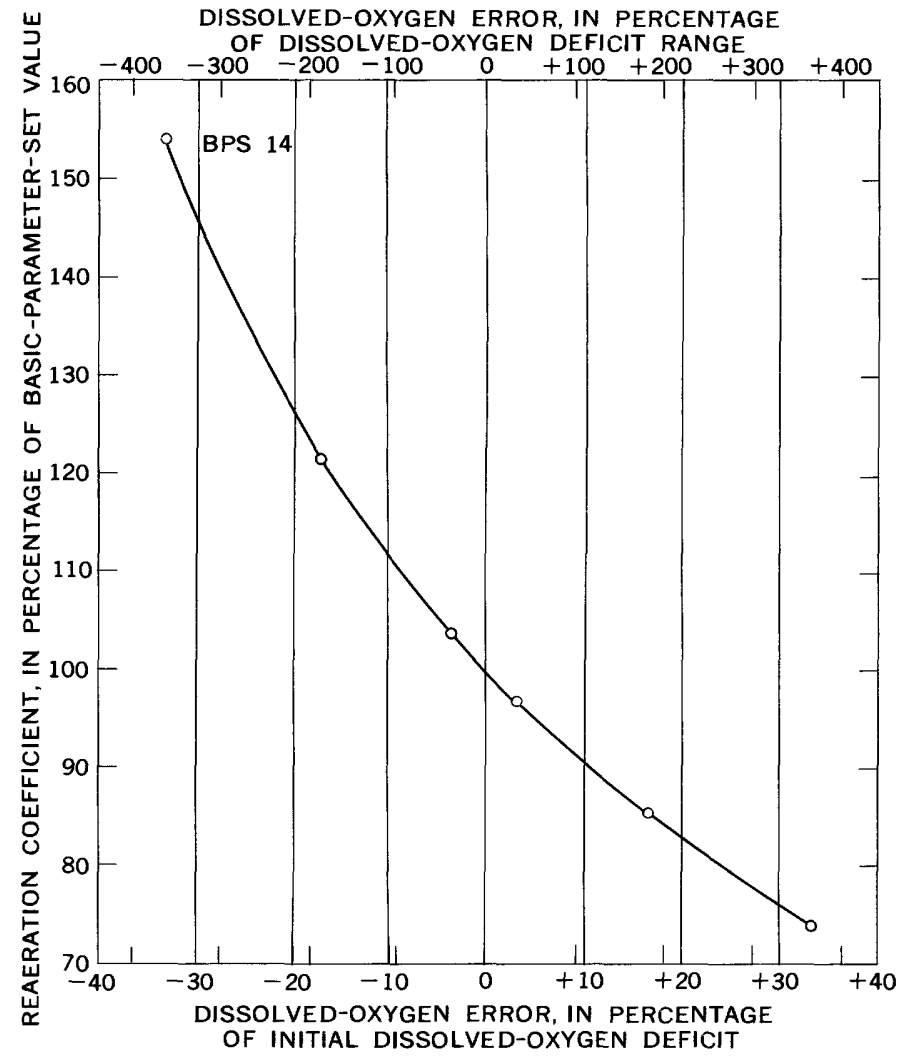

FIGURE 9. - Effect of a systematic error in DO measurement procedure on calculated reaeration coefficient, dissolvedoxygen balance technique.

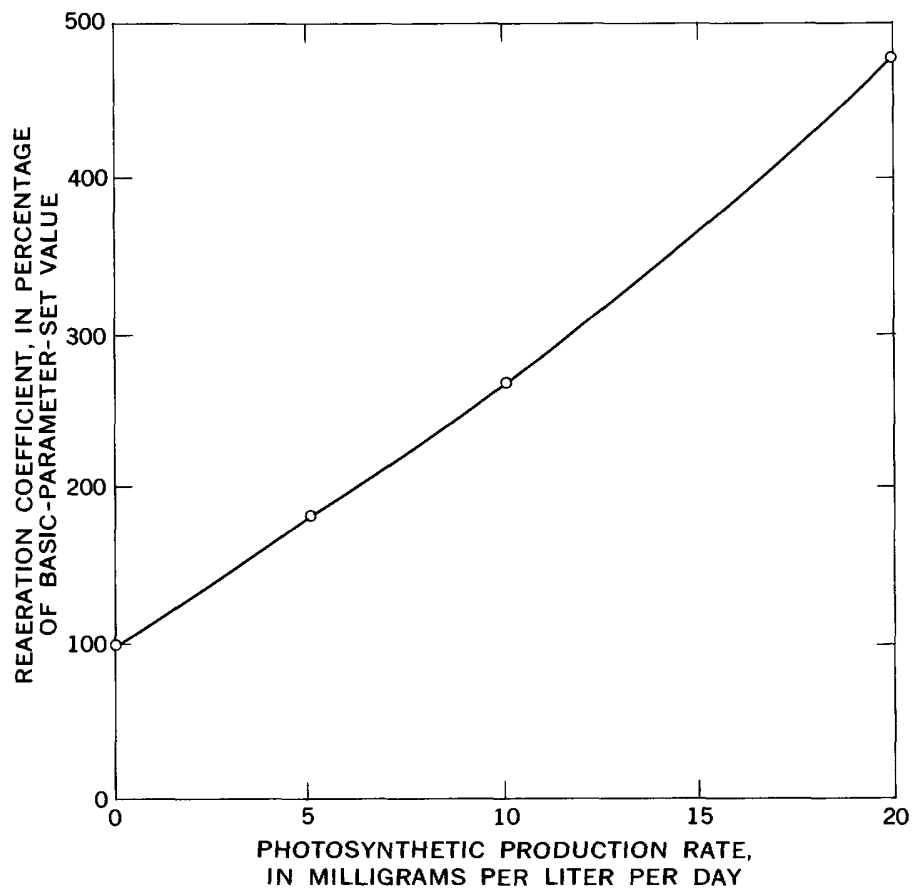

FIGURE 10. - Effect of ignoring photosynthetic-oxygen production rate, dissolved-oxygen balance technique. term in the equations. Then the $k_{2}$ was calculated for the same conditions except that photosynthesis was ignored. The effect is very definite; for example, for a photosynthetic production rate of $2 \mathrm{mg} / \mathrm{l}$ per day, the calculated $k_{2}$ is 130 percent of the BPS value of 1.0 days $^{-1}$. Thus, if a term for photosynthetic production is not included in the DO balance equation and this production is significant, the calculated $k_{2}$ will be significantly larger than the true value. The effect of photosynthesis was discussed previously by Camp (1965) and Lynch (1965).

The effect on the calculated reaeration coefficient of simultaneous errors in two or more of the parameters was investigated in this study to a limited extent, and it was found that the overall errors were in general not equal to the sums of the individual errors. Thus each individual error set must be investigated as an entity; however, this is beyond the scope of the present report.

From the results of the sensitivity analysis presented in figures 3 through 10 and in table 4 , several general conclusions are possible. Because of the limited scope of the sensitivity analysis, estimates of the errors will be qualitative only. It is expected in the calculation of the reaeration coefficient from equation 119 that:

1. If the BOD levels are small, and if attached or floating plants are present in significant quantities, then neglect of the photosynthetic production is probably the largest source of error in the calculation of the reaeration coefficient.

2. If the BOD levels are small, and if errors in the upstream and downstream DO deficit are independent, then these errors are probably the second largest source of error in the calculation of the reaeration coefficient.

3. For small to moderate upstream BOD levels, the errors in the calculated $k_{2}$ resulting from errors in $k_{1}$ and $L_{\mathrm{a}}$ are generally small but significant for $k_{2} \leqq 1.0$ days $^{-1}$. For an upstream BOD of greater than about $50 \mathrm{mg} / \mathrm{l}$, the errors in the calculation of $k_{2}$ resulting from errors in $k_{1}$ are especially significant.

4. The other parameters should cause no significant errors in the calculated $k_{2}$ unless $k_{2} \leqq 0.1$ $\operatorname{days}^{-1}$.

5. By summing the squares of the RMS errors for the average slope column of table 4 and taking the square root, an expected relative RMS error of 65 percent of a true $k_{2}$ value of 1.0 days $^{-1}$ is obtained.

6. If errors in the upstream and downstream DO deficits are the result of a systematic error in the DO measurement procedure or of the use of an 
incorrect DO saturation value, then these errors compensate each other to some extent so that the error in the calculated reaeration coefficient is not as great as it would be if the error had occurred independently in $D_{\mathrm{a}}$ or $D_{\mathrm{b}}$.

7. The effects of simultaneous errors in two or more parameters are not linearly additive, so that the results of this study cannot be used to determine the effects of more than one parameter error on the calculated $k_{2}$ values. Future studies should be directed to determining the sensitivity of the DO balance equation to errors in two or more parameters. The most ideal study would be to use Monte Carlo techniques, which would give the distribution function of the reaeration coefficient calculated from the DO balance equation for known distributions of the other parameters.

\section{DISTURBED-EQUILIBRIUM TECHNIQUE}

A rigorous statistical analysis of the disturbedequilibrium technique is presently impractical for the same reasons that such an analysis is impractical for the DO balance technique. However, a sensitivity analysis was performed, using the same approach as for the DO balance technique. The results of the sensitivity analysis and a comparison of the results of calculating reaeration coefficients from equations 139 and 140 are discussed in the following paragraphs.

The equations used in the sensitivity analysis were equation 140 written in the form

$$
f\left(k_{2}\right)=D_{\mathrm{b}}-D_{\mathrm{b}}{ }^{\prime}+\frac{p-p^{\prime}}{2.3 k_{2}}-10^{-k_{2} t}\left\{D_{\mathrm{a}}-D_{\mathrm{a}}{ }^{\prime}+\frac{p-p^{\prime}}{2.3 k_{2}}\right\}
$$

and a Newton-Raphson iterative solution as given by equation 149. The BPS values studied are listed in table 5 . These were chosen to correspond to the BPS used in the sensitivity analysis of the DO balance technique.

TABLE 5. - Basic parameter sets for the sensitivity analysis of the disturbed-equilibrium technique

\begin{tabular}{ccccccccc}
\hline $\begin{array}{c}\text { BPS } \\
\text { No. }\end{array}$ & $\begin{array}{c}k_{2} \\
\left(\text { days }^{-1}\right)\end{array}$ & $\begin{array}{c}t \\
(\text { days })\end{array}$ & $\begin{array}{c}D_{\mathrm{a}} \\
(\mathrm{mg} / \mathrm{l})\end{array}$ & $\begin{array}{c}D_{\mathrm{a}} \\
(\mathrm{mg} / \mathrm{l})\end{array}$ & $\begin{array}{c}p \\
(\mathrm{mg} / \mathrm{l} \\
\text { per day })\end{array}$ & $\begin{array}{c}p^{\prime} \\
\text { (mger day } / \mathrm{l})\end{array}$ & $\begin{array}{c}D_{\mathrm{b}} \\
(\mathrm{mg} / \mathrm{l})\end{array}$ & $\begin{array}{c}D_{\mathrm{b}} \\
(\mathrm{mg} / \mathrm{l})\end{array}$ \\
\hline 1 & 1 & 0.05 & 3 & 5 & 0 & 0 & 2.73 & 4.51 \\
2 & 1 & .05 & 3 & 5 & -1 & 0 & 2.73 & 4.46 \\
3 & 1 & .05 & 3 & 8 & 0 & 0 & 2.73 & 7.18 \\
4 & 1 & .05 & 3 & 8 & -1 & 0 & 2.73 & 7.14 \\
5 & .2 & .05 & 3 & 5 & 0 & 0 & 2.99 & 4.94 \\
6 & .2 & .05 & 3 & 5 & -1 & 0 & 2.99 & 4.89 \\
7 & 10 & .05 & 3 & 5 & 0 & 0 & .98 & 1.62 \\
8 & 10 & .05 & 3 & 5 & -1 & 0 & .98 & 1.59 \\
9 & 1 & .05 & 3 & 5 & -1 & 0 & 2.73 & 4.46 \\
10 & 1 & .05 & 3 & 5 & -5 & 0 & 2.73 & 4.27 \\
11 & 1 & .05 & 3 & 5 & -10 & 0 & 2.73 & 4.04 \\
12 & 1 & .05 & 3 & 8 & -5 & 0 & 2.73 & 6.95 \\
13 & 1 & .05 & 3 & 8 & -10 & 0 & 2.73 & $\mathbf{6 . 7 1}$ \\
\hline
\end{tabular}

The results of the sensitivity analyses of equation 151 are presented in figures 11 through 14 , in which the ordinates are in terms of percentage of the BPS value of the reaeration coefficient, and the abscissas are in the dimensions of the variable being investigated. In general, the sensitivity increases as the deficit range decreases.

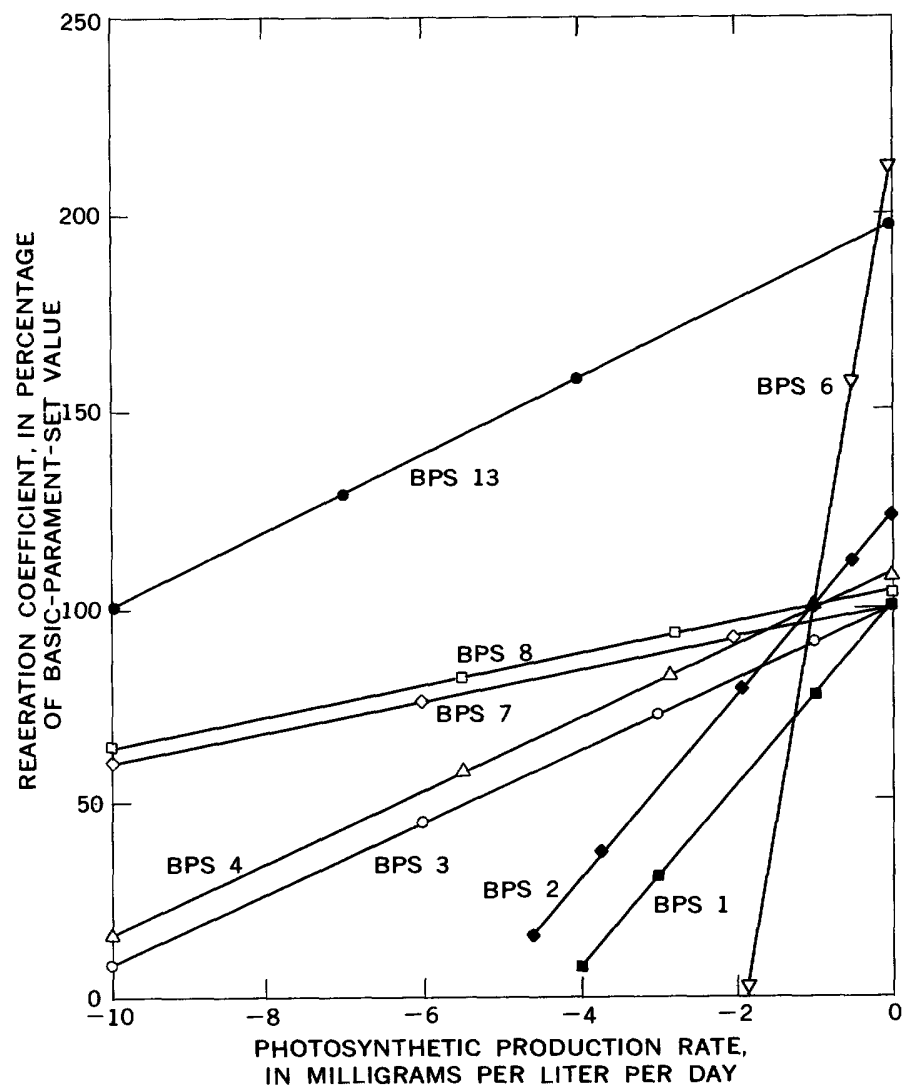

FIGURE 11. - Sensitivity of reaeration coefficient to photosynthetic-oxygen production rate, disturbed-equilibrium technique.

With the exception of the traveltime, two measurements of each parameter are necessary in the disturbed-equilibrium technique. Because of the additive nature of the variances, we have multiplied by 1.5 the estimated parameter errors presented previously, in table 2 , and used in the sensitivity analysis of the DO balance technique. These estimates of the parameter errors and the slopes of sensitivity-analyses plots for the disturbed-equilibrium technique (figs. 11 through 14) are presented in table 6. The possible errors in the calculated reaeration coefficient obtained from the different combinations of RMS and maximum errors and minimum, average, and maximum slopes are presented in table 7 . 


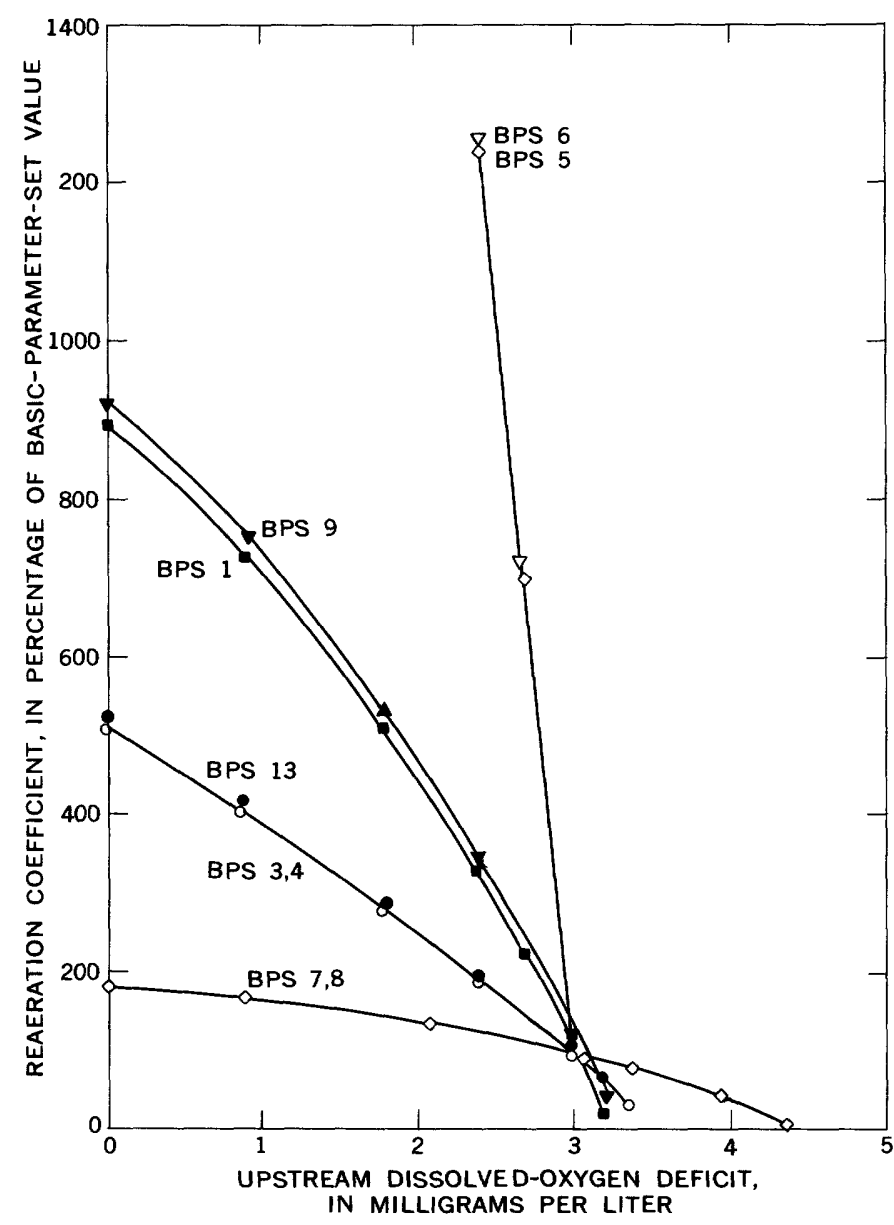

FIgURE 12. - Sensitivity of reaeration coefficient to upstream DO deficit, disturbed-equilibrium technique.

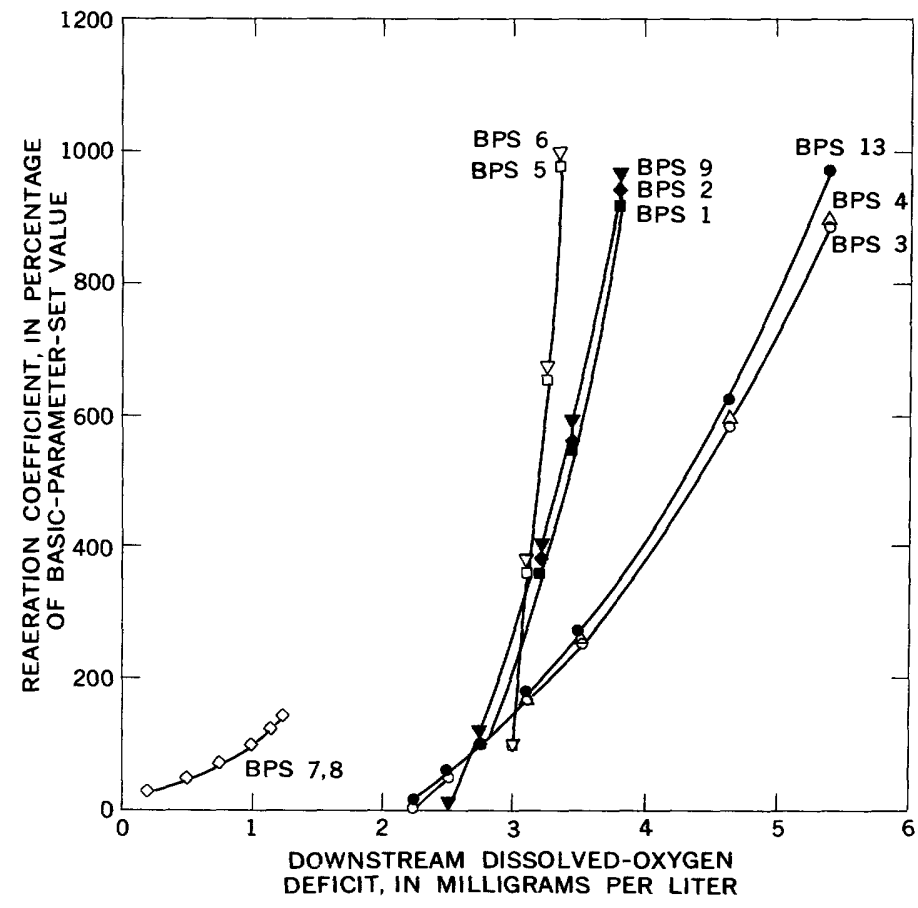

FIGURE 13. - Sensitivity of reaeration coefficient to downstream DO deficit, disturbed equilibrium technique.

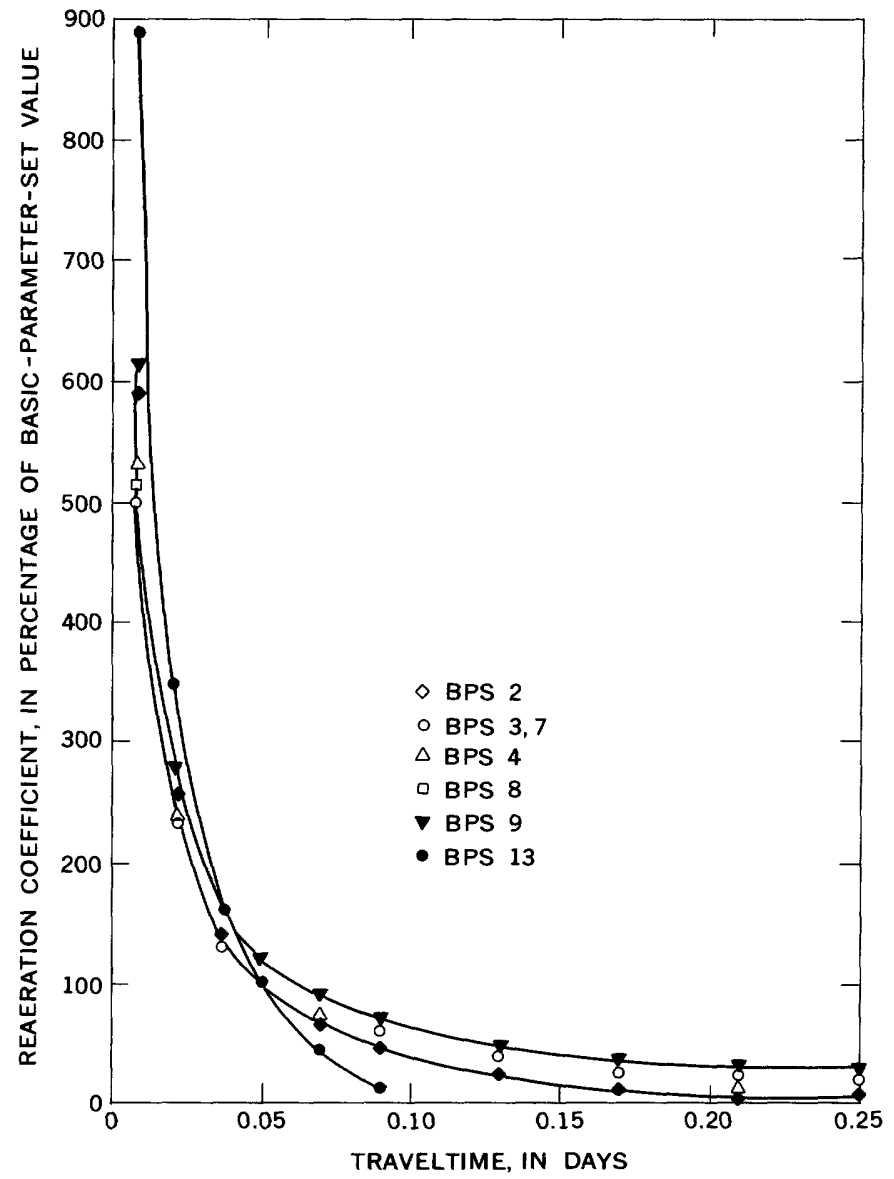

FIGURE 14. - Sensitivity of reaeration coefficient to traveltime, disturbed-equilibrium technique.

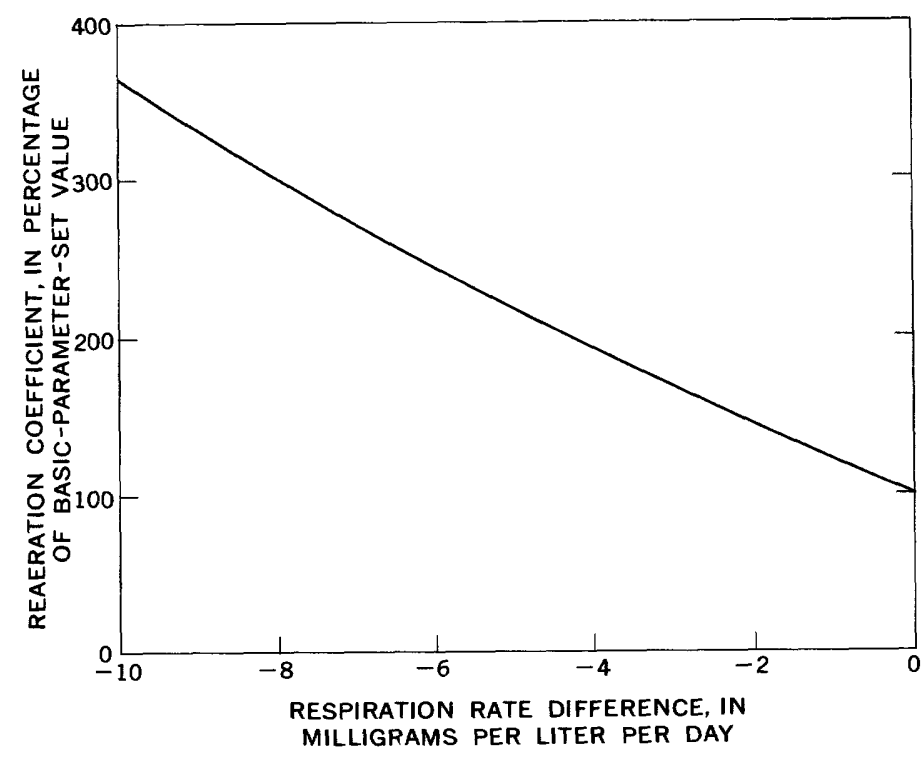

Figure 15. - Effect on calculated reaeration coefficient of ignoring a respiration rate difference, disturbed-equilibrium technique. 
TABLE 6. - Assumed parameter errors and slopes of sensitivity-analysis plots for disturbed-equilibrium technique

\begin{tabular}{|c|c|c|c|c|c|c|c|c|c|c|}
\hline Parameter & \multicolumn{4}{|c|}{$\begin{array}{l}\text { Assumed error } \\
\end{array}$} & \multicolumn{6}{|c|}{ Slope } \\
\hline $\begin{array}{l}p_{D_{\mathrm{a}}} \\
D_{\mathrm{b}} \\
t\end{array}$ & $\begin{array}{l}3.0 \\
.15 \\
.15 \\
.002\end{array}$ & $\begin{array}{l}\mathrm{mg} / 1 \text { per day } \\
\mathrm{mg} / 1 \\
\mathrm{mg} / 1 \\
\mathrm{day}\end{array}$ & $\begin{array}{l}15 \\
1.5 \\
1.5 \\
.005\end{array}$ & $\begin{array}{l}\mathrm{mg} / 1 \text { per day } \\
\mathrm{mg} / 1 \\
\mathrm{mg} / 1 \\
\mathrm{day}\end{array}$ & $\begin{array}{l}0.09 \\
1.5 \\
2.0 \\
2\end{array}$ & $\begin{array}{l}(\mathrm{mg} / 1)^{-1} \\
(\mathrm{mg} / 1 \text { per day })^{-1} \\
(\mathrm{mg} / 1 \text { per day })^{-1} \\
\text { days }^{-2}\end{array}$ & $\begin{array}{l}0.20 \\
2.5 \\
6.0 \\
20\end{array}$ & $\begin{array}{l}(\mathrm{mg} / 1)^{-1} \\
(\mathrm{mg} / 1 \text { per day })^{-1} \\
(\mathrm{mg} / 1 \text { per day })^{-1} \\
\text { days }-2\end{array}$ & $\begin{array}{l}0.40 \\
4.0 \\
14 \\
200\end{array}$ & $\begin{array}{l}(\mathrm{mg} / 1)^{-1} \\
\left(\mathrm{mg} / \mathrm{l}^{\mathrm{per}} \mathrm{day}\right)^{-1} \\
(\mathrm{mg} / 1 \text { per day })^{-1} \\
\text { days }^{-2}\end{array}$ \\
\hline
\end{tabular}

TABLE 7.-Errors in the calculated reaeration coefficient resulting from errors in parameter measurement, disturbedequilibrium technique

\begin{tabular}{|c|c|c|c|c|c|c|}
\hline \multirow{3}{*}{ Parameter } & \multicolumn{4}{|c|}{ Error in calculated $k_{2}$, in days ${ }^{-1}$} & \multirow{2}{*}{\multicolumn{2}{|c|}{ Maximum slope }} \\
\hline & \multicolumn{2}{|c|}{ Minimum slope } & \multicolumn{2}{|c|}{ Average slope } & & \\
\hline & $\begin{array}{l}\text { RMS } \\
\text { error }\end{array}$ & $\overline{\text { error }}$ & $\begin{array}{l}\text { RMS } \\
\text { error }\end{array}$ & $\underset{\text { error }}{\text { Maximum }}$ & $\begin{array}{l}\text { RMS } \\
\text { error }\end{array}$ & $\underset{\text { error }}{\text { Maximum }}$ \\
\hline $\begin{array}{c}p \\
D_{\mathrm{a}} \\
D_{\mathrm{b}} \\
t\end{array}$ & $\begin{array}{c}0.27 \\
.22 \\
.30 \\
.004\end{array}$ & $\begin{array}{c}1.35 \\
2.25 \\
3.0 \\
.01\end{array}$ & $\begin{array}{r}0.60 \\
.38 \\
.90 \\
.04\end{array}$ & $\begin{array}{l}3.0 \\
3.8 \\
9.0 \\
.10\end{array}$ & $\begin{array}{c}1.2 \\
.60 \\
2.1 \\
.40\end{array}$ & $\begin{array}{r}6.0 \\
6.0 \\
21 \\
1.0\end{array}$ \\
\hline
\end{tabular}

Figure 15 shows the effect of ignoring a difference in the respiration rates for the two levels of DO concentration of the disturbed-equilibrium technique. Reaeration coefficients were calculated from equation 151 for various respiration rate differences, and then the calculations were repeated with no respiration rate difference. It is apparent from figure 15 that the effect of ignoring the difference between respiration rates is definitely significant. For example, neglecting a respiration rate difference of $1.0 \mathrm{mg} / \mathrm{l}$ per day causes about 12 percent error in the calculated reaeration coefficient, and the error increases rapidly as the difference in respiration rate increases.

Either equation 139 or 140 can be used to calculate $k_{2}$ from measurements by the disturbedequilibrium technique when the respiration rates are different for the two levels of DO concentration. Equation 140 gives an integral reaeration coefficient, whereas equation 139 gives a reaeration coefficient based on the average DO deficit for the reach. Figure 16 shows the variation with respiration-rate ratio of $k_{2}$ as calculated from equation 139 for BPS 2, 4, and 13. A comparison of figures 15 and 16 shows that the sensitivity of the reaeration coefficient calculated from equation 140 to the difference in respiration rates is considerably less than the sensitivity of the reaeration coefficient calculated from equation 139 to the respiration-rate ratio.

In general, the respiration-rate ratio for a disturbed-equilibrium-technique experiment should not deviate too much from a value of 1.0. From figure 7 of Edwards, Owens, and Gibbs (1961), it is estimated that $R^{\prime}=0.85$ for a DO concentration change from 2 to $4 \mathrm{mg} / \mathrm{l}$, and that $R^{\prime}=0.65$ for

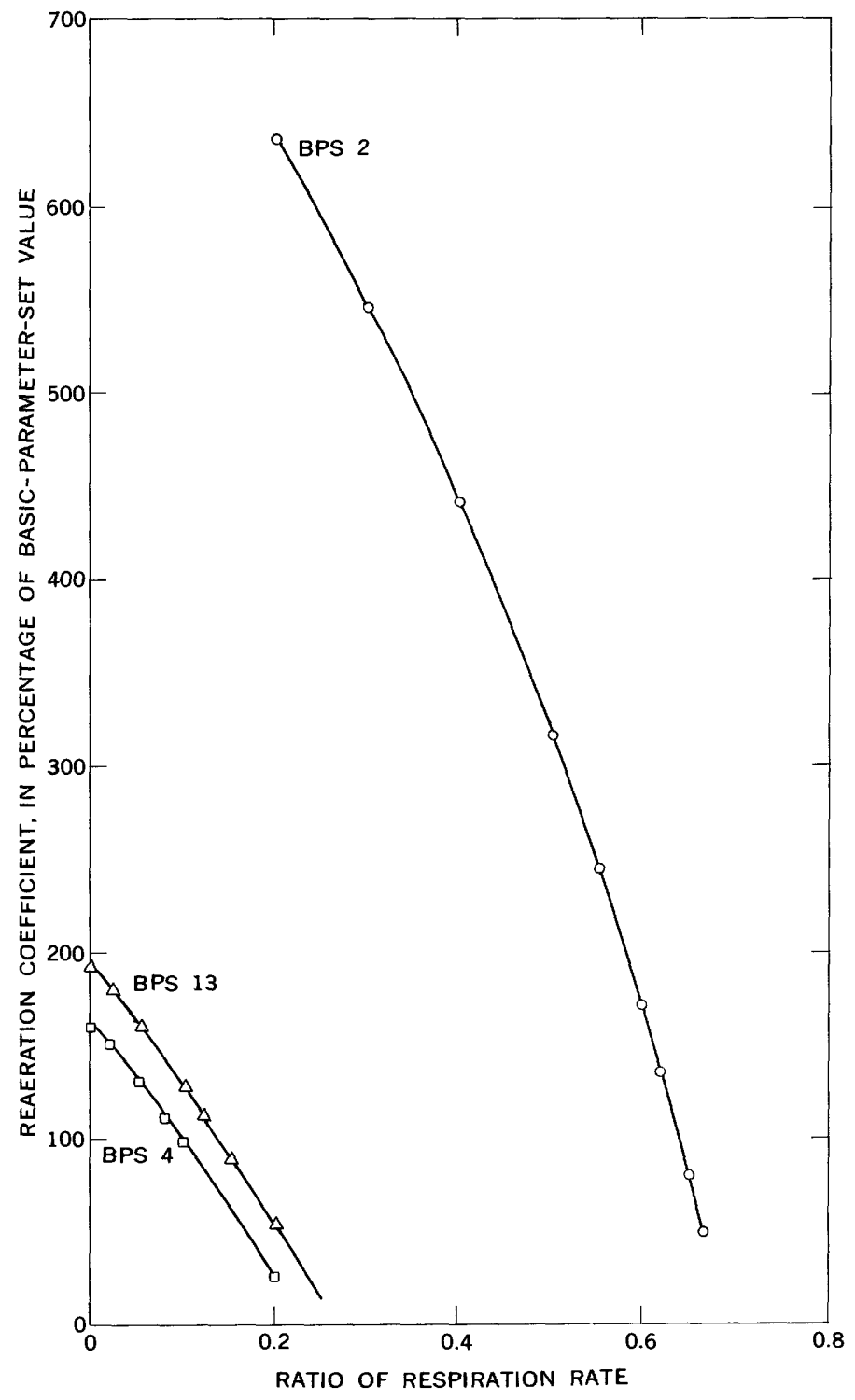

Figure 16. - Variation of calculated reaeration coefficient with respiration-rate ratio, disturbed-equilibrium technique.

a change from 2 to $10 \mathrm{mg} / \mathrm{l}$. From figure 16 , however, it is apparent that equation 139 cannot give the correct reaeration coefficient of BPS 2, 4, and 13 if $R^{\prime}>0.64$. Therefore, because the BPS values were chosen as representative of conditions in natural streams, the use of equation 140 is preferred. 
From the results of the sensitivity analysis presented in figures 11 through 16 and in table 7, several general conclusions are possible. These conclusions, as follows, are qualitative only, because of the limited scope of the sensitivity analysis.

1. Errors in all parameters of the disturbedequilibrium technique are significant in the calculation of the reaeration coefficient. The most significant is the error in the downstream DO deficit, followed by errors in the net photosynthetic production parameter, upstream DO deficit, and traveltime.

2. Neglecting a respiration-rate difference of $\mathbf{1 . 0}$ $\mathrm{mg} / \mathrm{l}$ per day causes about 12 percent error in the calculated reaeration coefficient; therefore, neglecting the effect of DO concentration on the respiration rate may be significant.

3. The use of equation 140 for disturbed-equilibrium-technique calculations is preferred.

4. The square root of the sum of the squares of the RMS errors for the average-slope column of table 7 yields an expected relative RMS error of 115 percent for a reaeration coefficient of 1.0 days $^{-1}$.

\section{TRACER TECHNIQUE}

Two major sources of error must be considered in an error analysis of the tracer technique. The first is the accuracy of the value of the ratio of the mass-transfer coefficient of the tracer gas to that of oxygen. The second is the accuracy of the procedures involved in measuring the mass-transfer coefficient of the tracer gas in an actual reaeration-coefficient determination.

The value of the mass-transfer coefficient ratio can cause errors in the calculated reaeration coefficient in two ways. First, there are errors inherent in the ratio because of errors in the laboratory procedures used to determine the ratio. Second, the conditions of turbulence and temperature and the presence of surfactants and pollutants in the measurement situation may be different from the conditions under which the ratio was determined, and this may result in a different ratio value.

The mass-transfer coefficient ratio for the krypton-oxygen gas pair was determined under a variety of laboratory conditions by Tsivoglou, O'Connell, Walter, Godsil, and Logsdon (1965) and by Tsivoglou (1967). The results of these tests are summarized in table 8 . Also shown in table 8 are the maximum and minimum reaeration coefficients observed in the two series of tests. A statistical analysis showed that the mean values were not significantly different at the 95-percent level of significance, and weighting the means in inverse proportion to their variances yielded a mean ratio of 0.83. The 95-percent confidence limits for the weighted mean were \pm 0.034 , and the most probable error was 0.011 , or about 1.3 percent of the mean ratio. Thus, the value of 0.83 may be regarded as an accurate estimate of the mass-transfer coefficient ratio for the krypton-oxygen gas pair.

TABLE 8. - Statistical data concerning measured gas-transfer coefficient

\begin{tabular}{|c|c|c|c|c|c|c|c|}
\hline \multirow{2}{*}{$\begin{array}{l}\text { Transfer } \\
\text { coefficient } \\
\text { ratio }\end{array}$} & \multirow{2}{*}{ Mean } & \multirow{2}{*}{ Minimum } & \multirow{2}{*}{ Maximum } & \multirow{2}{*}{ Median } & \multirow{2}{*}{$\begin{array}{l}\text { Standard } \\
\text { deviation }\end{array}$} & \multicolumn{2}{|c|}{ Range of $K_{2}\left(\right.$ days $\left.^{-1}\right)$} \\
\hline & & & & & & Minimum & Maximum \\
\hline$\frac{K_{2_{G}}^{\dagger}}{K_{2}}$ & 0.82 & 0.76 & 0.91 & 0.81 & 0.04 & 1.5 & 12.5 \\
\hline$\frac{K_{2_{G}} \downarrow}{K_{2}}$ & .846 & .789 & .909 & .846 & .045 & 4.4 & 68.6 \\
\hline
\end{tabular}

†Tsivoglou, O'Connell, Walter, Godsil, and Logsdon (1965).
tTsivoglou (1967).

The effect of different conditions on the ratio was investigated extensively by Tsivoglou, O'Connell, Walter, Godsil, and Logsdon (1965) and Tsivoglou (1967). They measured mass-transfer coefficient ratios in two different experimental systems for seven different gases for transfer both into and out of the water. They also used a range of temperature and turbulence conditions that produced reaeration coefficients comparable to the upper range of reaeration coefficients observed in natural streams. They concluded that the mass-transfer coefficient ratio was not influenced significantly by (1) temperature in the range from $10^{\circ}$ to $30^{\circ} \mathrm{C}$, (2) the degree of turbulent mixing over a wide range of mixing conditions, (3) the presence or absence of a broken water surface, and (4) the direction of gas transfer.

In addition to this experimental evidence, some of the conceptual models discussed previously and a theoretical argument presented by Tsivoglou (1967) suggest that the transfer coefficient ratio is equal to the ratio of molecular diffusivities. Thus, this observation also suggests that the transfer coefficient ratio should be a constant, if differences in the temperature dependences of the diffusivities are neglected.

The accuracy of the procedure used to measure the mass-transfer coefficient of the tracer gas in an actual reaeration-coefficient determination depends on the accuracy of the nuclear counting techniques, the measurement of the flow time, and sampling errors. The counting techniques used by Tsivoglou (1967) involved splitting each sample into three parts and determining the number of 
counts of each sample for at least two 20-minute periods. Tsivoglou (1967) reported,

This procedure, involving considerable replication and long total counts, provided excellent counting statistics, and a high level of accuracy for river samples whose count rates might be in the neighborhood of only twice the background count rate. Flow time in the tracer technique is determined from measurements of the concentrations of the water-soluble fluorescent dye and, hence, should be very accurate. Furthermore, the flow time determined by this procedure should be identical with the flow time for the conservative liquid and gas tracers. Errors resulting from sampling should be small because the only quantity determined from the samples is the ratio of the concentrations of the gas and liquid tracers. Care must be exercised, however, to insure that the gas tracer is not lost from the samples during handling and counting.

The overall accuracy of the tracer technique is difficult to estimate. A qualitative indication of the reproducibility of $k_{2}$ values determined from the tracer technique can be obtained from the work of Tsivoglou and others $(1967,1968)$. This work included two or three repetitions of $k_{2}$ measurements for 14 river reaches. The average deviation from the mean for nine reaches was less than \pm 10 percent; the average deviation was less than \pm 6 percent for six of the reaches; and the maximum deviation for any reach was \pm 18.2 percent. Because two days generally elapsed between measurements on a specific reach, some of the variation could have been the result of actual changes in the reaeration coefficient. Thus, on the basis of these limited data, errors in reaeration coefficients determined by the tracer technique may be of the order of \pm 15 percent of the mean.

\section{LABORATORY MEASUREMENTS}

In the laboratory, most of the sources and sinks in the DO balance can be eliminated, so that the complete DO balance, equation 119 , reduces to the simple equation 133 for a laboratory flume. Hence, laboratory flume measurements of the reaeration coefficient should provide a limit on the reproducibility that can be expected from the DO balance technique in general. Unfortunately, the literature does not contain much information concerning reproducibility of reaeration-coefficient measurements in flumes. It does contain, however, some information for mixing-tank studies, an experimental situation considerably simpler than the flume situation because only one plot of $\log D$ versus time is involved. Dobbins (1964a) reported measurements on three different gases at constant turbulence conditions in a mixing tank; in two cases, four repetitions were made, and the resulting variances were 3.6 percent and 8.6 percent of the mean. In one case, involving seven repetitions, the variance was 8.5 percent of the mean. Isaacs and Gaudy (1968) reported measurements in a "simulated" stream which in essence was a mixing tank with moving walls, used in an attempt to duplicate velocity conditions in streams. They reported two situations where five repetitions were made in which the variance was 6.3 and 6.9 percent of the mean. In five situations where three repetitions were used, the variance was from 1.6 to 4.1 percent of the calculated $k_{2}$ value. Dobbins' $k_{2}$ determinations were based on measurements of the volume of gas absorbed, whereas Isaacs and Gaudy's were made using the azide modification of the Winkler test. If one compares the average variance of about 5 percent of Isaacs and Gaudy's measurements with the 0.1 percent of $C_{s}$ reproducibility expected in the DO measurements with the Winkler method, the comparison indicates that as the complexity of the measurement procedure increases, the error increases considerably, so that the variance of flume measurements would be expected to be even larger than 5 percent. Furthermore, the comparison suggests that the accuracy of the DO measurement procedure should not be considered as indicative of the accuracy of the complete determination procedure for the reaeration coefficient.

The difference between the reproducibility of the Winkler method for DO determination and the expected reproducibility of flume measurements shows that other factors are important in the flume measurement procedures. These factors may be errors in reproducing or measuring the hydraulic conditions describing the flow. In addition, another factor not previously considered by researchers is the possibility of a corrosion reaction in the flume system.

Many laboratory flumes are constructed from metals, and because metals corrode or react with oxygen to varying degrees, a DO sink may be present that the researcher has not considered. The reaeration coefficient calculated by the procedure illustrated in figure 2 may be incorrect when a corrosion reaction is occurring in the channel. Furthermore, the magnitude of the error has been shown to depend on the relative magnitudes of the reaeration coefficient and the rate constant for the corrosion reaction and also on the initial DO deficit. (See appendix A.)

Therefore, the first step of any laboratory study of the reaeration coefficient should probably be 
the determination of the significance of possible corrosion reactions together with a determination of the reproducibility of the experimental measurements. The reproducibility of the measurement procedure can be determined by replication of the experimental runs, and the significance of possible corrosion reactions can be determined by the procedures outlined in appendix $\mathbf{A}$.

The presence of an oxygen-consuming reaction in the laboratory equipment shows one way in which systematic errors can be introduced into flume measurements of the reaeration coefficient. This might explain one of the puzzling facts present in the literature on reaeration in flumes. This puzzle is the inconsistency between prediction equations obtained from data taken in two different but supposedly hydraulically equivalent flumes. The work of Krenkel (1960) and Thackston (1966) illustrates this inconsistency. The flumes used by these investigators were not significantly different hydraulically, except that Krenkel's was 1.0 foot wide and Thackston's was 2.0 feet wide. The results, however, obtained from their studies over similar ranges of hydraulic variables may be given as (Thackston, 1966):

$$
\text { (Krenkel) } k_{2}=0.000469 \frac{u_{*}}{H}
$$

and

$$
\text { (Thackston) } k_{2}=0.000215 \frac{u_{*}}{H} \text {. }
$$

The constants in the equations differ by a factor of 2.2 .

The reasons for the discrepancy between these equations is not known. However, it might be due to the systematic error introduced by an oxygenconsuming reaction proceeding in the channel of Qne or the other of the experimental flumes.

Another possibility for the discrepancy is the failure to include some pertinent hydraulic variable in the equation-for example, the width-depth ratio to account for the different widths. However, Thackston and Krenkel (1969a) wrote: "It is possible that some sort of wall effect is involved, inasmuch as the values of the width-to-depth ratio were essentially the same in both studies***." Additional experimental work will be necessary to determine the effect of the width-depth ratio on the reaeration coefficient.

\section{PREDICTION OF THE REAERATION COEFFICIENT}

None of the presently available models of the oxygen-absorption process in open-channel flows are well enough developed-or have been sufficiently verified - so that they can be used to accurately predict reaeration coefficients from the mean hydraulic parameters. Because of this, one must rely for the prediction of reaeration coefficients on empirical regression equations based on experimental data. The mean parameters commonly available for use as the independent variables in such equations are width, depth, slope, and velocity. Another parameter which has been used as an independent variable in $k_{2}$ prediction equations is the longitudinal-dispersion coefficient, $D_{x}$, but because of a scarcity of experimental $D_{x}$ data, this parameter has not been used herein.

The purpose of this section is to (1) present a summary of the known experimental data concerning the reaeration coefficient in open-channel flows, (2) evaluate the presently available equations for prediction of reaeration coefficients, and (3) present what we consider to be the most reasonable prediction equations that can be obtained by regression analysis from the available data.

\section{REAERATION-COEFFICIENT DATA SETS}

The reaeration-coefficient data sets available in the literature can be judged against two basic criteria. The first of these is the accuracy with which the basic data were measured and the carefulness with which the coefficients were calculated. The measurement techniques used must be considered, and if the DO balance technique or the disturbed-equilibrium technique is used, the effects of all neglected parameters must be evaluated.

The second criterion is the completeness of the data in the set. Specifically, this concerns the number of independent variables reported and the ranges of the independent and dependent variables.

From the points of view of scale and of datacollection methods, the reaeration-coefficient data fall into two general classes. These classes are data from laboratory flumes and data from natural streams. The flume data were in general collected at flow depths one to two orders of magnitude lower than the field-data flow depths, and at slopes one to two orders of magnitude higher. In terms of scaling, the data of Owens, Edwards, and Gibbs (1964) to some extent bridge the gap between the rest of the field data and the flume data.

FIELD DATA

The best available data were presented by Churchill, Elmore, and Buckingham (1962). The 
DO balance technique was used, for the most part under such idealized conditions, and with such attention to detail that a high degree of accuracy is expected in the reaeration coefficients. These measurements were made in river reaches downstream from deep storage impoundments. A DO deficit was present because the water released was low in DO concentration as a result of prolonged storage under thermally stratified conditions. For the reservoirs studied, the $\mathrm{BOD}$ of the water was satisfied during storage, so that it was zero at release time.

Over the study period on each reach, the stream discharge was held constant by keeping the discharge constant at the upstream hydroelectric plant. The discharge was determined at local stream gaging stations, and the mean velocity was determined by dividing the discharge by the average cross-sectional area. The flow time was determined by dividing the length of the test reach by the mean velocity. Because the test reaches were carefully selected for uniformity, and because cross sections were measured at intervals roughly equivalent to the stream width, the accuracy of this method of determining the flow time is probably consistent with the accuracy of the other steps in the DO balance procedure used for the calculation of $k_{2}$.

The Winkler method, with a special end-point determination procedure which produced a standard deviation of only $0.007 \mathrm{mg} / \mathrm{l}$, was used to determine the DO concentrations. The measured DO concentrations showed no variation in the vertical, but for many cross sections, the lateral DO variation exceeded the variation in $\mathrm{DO}$ between the ends of the test reach. This necessitated taking DO samples at several points in the cross section and using the average of the measurements as the cross section DO concentration. For most cross sections, seven samples were used.

For all but seven of the 30 reaches studied, Churchill, Elmore, and Buckingham (1962) believed that except for $k_{2}$, all of the parameters in the DO balance equation were negligible. For the seven reaches they felt that correction was necessary for the photosynthesis and respiration of attached plants. The corrections were made using laboratory measurements of the photosynthesis and respiration rates of plants attached to rocks removed from the river bottom. For reasons discussed previously, and as pointed out by Hull and DeFilippi (1963), this type of correction is dubious, so that these seven values are less reliable than the other values reported. However, the problem was recognized, and an attempt was made to compensate for it. In addition, the corrections were for less than 25 percent of the calculated coefficients, which, as will be shown later, is of the order of magnitude of the scatter around the best-fit regression equations obtainable from the field data.

The data obtained by Churchill, Elmore, and Buckingham (1962) are listed in table 18 of appendix B. In terms of the commonly measured mean hydraulic parameters, this data set is considered complete, because all the variables $U, H, S$, and $W$ are reported. The ranges of the measured variables are listed in table 9. These data are representative of moderate-size streams.

TABLE 9. - Ranges of experimental data from the literature

\begin{tabular}{|c|c|c|c|c|c|c|c|c|c|c|}
\hline \multirow{2}{*}{ Reference } & \multicolumn{2}{|c|}{$\begin{array}{c}k_{2} \\
\left(\text { days }^{-1}\right)\end{array}$} & \multicolumn{2}{|c|}{$\underset{\text { (feet) }}{H}$} & \multicolumn{2}{|c|}{$\underset{(\text { fps) }}{U}$} & \multicolumn{2}{|c|}{$\underset{\text { (feet) }}{W}$} & \multicolumn{2}{|c|}{$\begin{array}{c}S \times 10^{4} \\
(\mathrm{ft} \text { per ft) }\end{array}$} \\
\hline & Maximum & Minimum & Maximum & Minimum & Maximum & Minimum & Maximum & Minimum & Maximum & Minimum \\
\hline $\begin{array}{l}\text { Churchill and } \\
\text { others (1962) }\end{array}$ & 5.558 & 0.225 & 11.41 & 2.12 & 5.00 & 1.85 & 667 & 90 & 23.514 & 1.2571 \\
\hline $\begin{array}{l}\text { Owens and } \\
\text { others (1964) }\end{array}$ & 49.17 & .31 & 2.44 & .39 & 1.83 & .13 & 39.8 & 11.0 & 106.0 & 1.56 \\
\hline 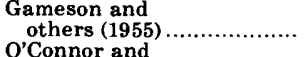 & 57.7 & 34.3 & .42 & .34 & .73 & 1.12 & 5.7 & ..... & ..... & ..... \\
\hline Dobbins (1958)† ................... & .92 & .14 & 24.2 & 4.0 & 4.20 & .19 & ..... & $\ldots .$. & 1.97 & .68 \\
\hline others $(1967,1968) \ldots \ldots \ldots \ldots$ & 3.373 & .646 & 3.11 & 1.16 & .636 & .286 & 110 & 58 & & \\
\hline Thackston $(1966) \ldots \ldots \ldots \ldots \ldots$ & $\begin{array}{r}52.50 \\
1153\end{array}$ & 5.78 & .232 & .052 & 2.320 & .365 & 2.0 & $\ldots .$. & 201.2 & 6.5 \\
\hline $\begin{array}{l}\text { Krenkel (1960)................. } \\
\text { Negulescu and }\end{array}$ & & 10.63 & & & & & & ..... & 239.9 & \\
\hline Rojanski (1969) ................ & 18.70 & 8.64 & .492 & .164 & 1.902 & .656 & .66 & $\ldots .$. & $\ldots \ldots$ & $\ldots$. \\
\hline
\end{tabular}

$\nmid$ Streeter and Phelps (1925) data only.

The reaeration-coefficient study presented by Owens, Edwards, and Gibbs (1964) was performed under conditions much less ideal than those prevailing for the study by Churchill, Elmore, and Buckingham (1962). In 11 of the 32 cases presented, Owens, Edwards, and Gibbs noted that either considerable weed growth or mild organic pollution was present. However, they did not state whether phytoplankton were present. The presence of plants is an important factor, because the disturbed-equilibrium technique was used to calculate the reaeration coefficients, and 
different DO consumption rates of the plants at the two DO levels used in this test could have significantly influenced the calculated reaeration coefficients.

In the study by Owens, Edwards, and Gibbs (1964), the discharge was determined by a dilution technique. The flow time was determined from the time of passage of the centroid of the tracer pulse through the reach. The reliability of the reported depths cannot be determined because the frequency of cross-sectioning was not reported. DO measurements were made using the Alsterberg modification of the Winkler method.

The paper by Owens, Edwards, and Gibbs (1964) contains two sets of data. The first set consists of the $32 k_{2}$ values determined by these workers. Eleven of the $k_{2}$ values were corrected for oxygen consumption by the bottom mud or for oxygen consumption by attached plants. This data set does not include the stream width, but this parameter can be calculated. The second data set, consisting of six $k_{2}$ values, was taken from Gameson, Truesdale, and Downing (1955). In this data set, neither the width nor slope values are given, but width values can be calculated. The two data sets are given in tables 19 and 20 in appendix $B$, and the ranges of the variables are given in table 9 . The hydraulic parameters would be considered typical for steep, shallow streams.

A summary of the pre- 1958 field data was presented by O'Connor and Dobbins (1958). The data consist for the most part of those of Streeter and Phelps (1925), screened by O'Connor and Dobbins (1958) to minimize the effects of algae and sludge deposits and modified so that the reported depth values approximate the true stream depth rather than the distance above mean low water.

The modified Streeter and Phelps (1925) data must be viewed with some caution, because they were obtained from a river containing organic pollution and probably phytoplankton, and $k_{2}$ values were computed from the Streeter-Phelps form of the DO balance equation, which ignores all but the suspended and dissolved BOD oxidation and the surface reaeration. In many of the test reaches the other terms in the DO balance equation probably would have influenced the calculated $k_{2}$ values, had they been available for use in a more general form of the DO balance equation.

The data of Streeter and Phelps (1925) as modified by O'Connor and Dobbins (1958) are reproduced in table 21, appendix $B$. The ranges of the variables are given in table 9 . The data set is incomplete in that width values are not given. The StreeterPhelps data typify moderate-size rivers on gentle slopes. Table 22 of appendix B lists data collected by O'Connor and Dobbins from sources other than Streeter and Phelps. Stream width is not included in this data set, nor in three cases is stream slope. Because the sources of the individual data points vary, the ranges of the variables in this data set have not been included in table 9 .

The only reaeration-coefficient data for streams that have been determined using the tracer technique are those given by Tsivoglou and others $(1967,1968)$. The data are rather limited in scope because the prime purpose of the study was not $k_{2}$ determination, but verification of the measurement technique itself.

In the study by Tsivoglou and others $(1967,1968)$, the traveltime was determined from the time of passage of the dye peak between the sampling stations. The stream was cross-sectioned every 1000 feet, but as Tsivoglou stated (1967, p. 69), "The stream was characterized by a series of riffles and pools and an associated considerable range of velocities and depths," so the average depth measurements are questionable. The nuclear counting techniques used were highly reproducible, and sufficient replications were used so that even at low krypton concentrations the tracer-gas to liquid-concentration ratio should have been very accurate. The tracers were introduced at the centerline of the injection cross-section and sampled from the flow at the centerline of each sampling cross section. In light of the results of the study by Churchill, Elmore, and Buckingham (1962), this sampling procedure would seem to be questionable if the $k_{2}$ values obtained are to be representative of the entire cross section. Despite the questionable sampling technique, the reproducibility of calculated $k_{2}$ values was good, with a maximum deviation from the mean of 18 percent and with nine of 14 values having an average deviation from the mean of less than 10 percent.

The experimental data obtained by Tsivoglou and others $(1967,1968)$ are listed in table 23 , appendix $B$. The channel slope is not given, and the width value was obtained by the present authors by dividing the depth-velocity product into 82.5 cfs, the average discharge for the time period. The ranges of the individual variables are listed in table 9.

\section{FLUME DATA}

In all the laboratory studies reported herein, the flumes used were the recirculating type, and 
the water in the flume was deaerated with sodium sulfite before the start of the experimental measurements.

Thackston (1966) used a flume 60 feet long and 2 feet wide, and three different bottom roughnesses. All DO measurements were made using the Azide Winkler method, and the traveltime measurements were made using dissolved sodium chloride as a tracer. The sampling stations in the flume were 42 feet apart; at each station, 10 samples were taken at 2-minute intervals. With at least two of the bottom roughnesses used, an oxygen-consuming reaction may have influenced the calculated reaeration coefficients. See appendix A for a discussion of this problem.

The data of Thackston (1966), also reported by Thackston and Krenkel (1969a), are given in table 24 , appendix B. The data set is complete, in that all the mean hydraulic parameters commonly measured are available. The ranges in the values of the variables are listed in table 9.

The flume used by Krenkel (1960) was 60 feet long and 1 foot wide, and the bottom roughness consisted of expanded metal mesh. In terms of experimental methods and techniques, and in terms of quality of measurements, the study was comparable to that by Thackston (1966). Again, oxidation reactions within the flume channel may have influenced the calculated reaeration coefficients. See appendix A.

The data of Krenkel (1960), which were also used by Krenkel and Orlob (1963), are given in table 25, appendix B. Again, all the commonly measured mean hydraulic parameters are listed, so the data set is considered to be complete. The ranges in values of the variables are reported in table 9 .

The flume used by Negulescu and Rojanski (1969) was 66 feet long and 0.66 foot wide and had a 40 -foot test section. The bed roughness consisted of $1-\mathrm{mm}$ sand grains cemented to the flume floor. The DO measurements were made using the Winkler method. A 20-minute measuring period was used, and about 10 samples were collected at each sampling station.

Negulescu and Rojanski (1969) tabulated only eight of the 18 values they determined. These are listed in table 26, appendix B. Values for slope and temperature were not included in the original reference, so a temperature of $20^{\circ} \mathrm{C}$ was assumed. The ranges in values of the variables are listed in table 9.
EVALUATION OF REAERATION.COEFFICIENT PREDICTION EQUATIONS

The conceptual models discussed earlier have, in general, not been carried to the point where they can be used by themselves to predict reaeration coefficients from the mean stream parameters of width, depth, slope, and velocity. The most useful reaeration coefficient prediction equations are, therefore, those obtained from the semiempirical models. These equations have coefficients derived in some fashion from field data, or derived entirely by least-squares regression analysis from field data. Of course, such prediction equations should be used with caution in situations for which the mean stream parameters are outside the range of data from which the prediction equations were derived.

In this section, we will, for the prediction equations available in the literature, evaluate their performance over the entire range of available data. We will also assess on a common basis the performance of the various equations when applied to the data sets from which the equations were derived.

The basis chosen for comparison of the performance of the various prediction equations is the standard error of estimate. For a prediction equation yielding an estimated $\left(k_{2}\right)_{e}$ for which the calculated reaeration coefficient is $\left(k_{2}\right)_{c}$, the standard error of estimate, $E_{S}$, is

$$
E_{S}=\sqrt{\frac{\sum_{i=1}^{n}\left[\left(k_{2}\right)_{e}-\left(k_{2}\right)_{c}\right]_{i}^{2}}{n}},
$$

where $n$ is the number of $k_{2}$ values used. Another definition of the standard error of estimate which is convenient to use in conjunction with leastsquares prediction equations derived from the logarithms of $k_{2}$ is

$$
E_{S L}=\frac{\sqrt{\sum_{i=1}^{n}\left[\log _{10}\left(k_{2}\right)_{e}-\log _{10}\left(k_{2}\right)_{c}\right]_{i}^{2}}}{n} .
$$

The term $E_{S L}$ can be expressed as a percent error, $E_{P}$, defined as

$$
E_{P}=100\left[1-10^{-E_{S L}}\right],
$$

where $E_{P}$ is the percent standard error of estimate in terms of $\left(k_{2}\right)_{e}$. The term $E_{P}$ will always be between 0 and 100 percent. When compared with $E_{S}$ as a percent, it is a conservative estimate of the percent error of the prediction equation. It must be emphasized that $E_{P}$ is not equal to the percent standard error of estimate that would be calculated from the regression of $k_{2}$ (rather than the transformed data $\log k_{2}$ ). 
In table 10 are reported the quantities $E_{S}$ and $E_{P}$ for 13 of the equations discussed previously. For each of the equations, $E_{S}$ and $E_{P}$ have been computed-first, for the data sets used by the original authors, and second, for all of the data sets discussed in the previous section which are sufficiently complete for use in the particular equation being evaluated. Table 10 also contains, for both groups of data, the number of data points and the ranges of the individual variables.

On the basis of the percent standard error of estimate, the equations which seem to fit best their original data sets are equation 96 , with a standard error of estimate of 15 percent, and equation 101, with a standard error of estimate of 25 percent. These equations were presented, respectively, by Krenkel (1960) and by Thackston (1966). The relatively good fit of these equations is not surprising, because they were obtained from laboratory studies in which the interfering factors and the hydraulic variables could be much better controlled than under the field conditions represented by most of the other equations.

Again on the basis of the percent standard error of estimate, the equations which best fit the entire range of available data are equation 61 , with a standard error of estimate of $\mathbf{4 3}$ percent; equations 101 and 102, with standard errors of estimate of 45 percent, and equation 54, with a standard error of estimate of 46 percent. Equation 61 , given by Dobbins (1964b), was derived from a consideration of two sets of field data and one set of flume data; equation 101, presented by Thackston (1966), was derived from flume data; equation 102 , again from Thackston (1966), was derived from flume data, along with two sets of field data; equation 54, presented by O'Connor and Dobbins (1958), was derived without resorting to any experimental data. As might be expected, two of the best-fit equations were derived from data which covered essentially the same range as the data in appendix $\mathbf{B}$.

In the 10th column under "Total applicable data" in table 10 , it is seen that five of the equations, when applied to the entire available data range, have standard errors of estimate greater than 100 per day. With the exception of equations 56 and 89, these five equations were derived from river data and give much too high $k_{2}$ predictions at the shallow depths used in the flume studies. This fact illustrates the caution which must be used when applying $k_{2}$ prediction equations to situations in which the mean flow parameters are well outside the range of the data from which the prediction equations were obtained. Further caution is indicated when it is realized that none of the values of $E_{S}$ listed in column 10 are less than 16 per day, a considerable magnitude when compared with the $k_{2}$ values of less than 5 per day usually determined for natural streams.

The reason for the large $E_{S}$ values given in the 10th column under "Total applicable data" in table 10 is further emphasized by reference to figure 17 . In this figure, all the equations of table 10 have been plotted for an assumed slope of 0.0001 feet per foot and a mean velocity of $1.0 \mathrm{fps}$. The square ruled on the graph delimits the range of experimental data used. For the smaller depths at the extreme left edge of the depth range, there is sometimes more than two orders of magnitude difference between a given pair of prediction equations. At the smaller depth values, the experimental data fall into a range representative of the bottom six prediction equations shown in the figure, so that large standard errors of estimate would be expected for the prediction equations which yield large $k_{2}$ values at shallow depths.

In general, two different sets of equations are apparent in figure 17: those derived from flume data, which have slopes on the order of $-1 / 2$, and those derived from field data, which have slopes on the order of $-3 / 2$. It becomes apparent that there is a basic difference between the data sets obtained in the two different situations. Because of this, then the use of equations derived from flume data for the prediction of reaeration coefficients in natural streams is dubious. This matter is discussed further in the following sections.

\section{DEVELOPMENT OF A REAERATION-COEFFICIENT PREDICTION EQUATION}

As discussed previously, none of the available prediction equations can be considered applicable to the entire range of available data. Assuming the data to be bias-free, there are two possible reasons for this. First, none of the available equations were derived from a wide enough data range, and second, and more likely, some basic difference exists between the two data sets which precludes writing an equation which is applicable to the entire range of data. In this section, these alternatives are investigated using regression-analysis procedures. The data used are those discussed previously. The criterion for judging the best equation to use is the standard error of estimate of the prediction equation as obtained from the 62 field and (or) 110 flume data sets which contain all the variables $U, H, W$, and $S$. 


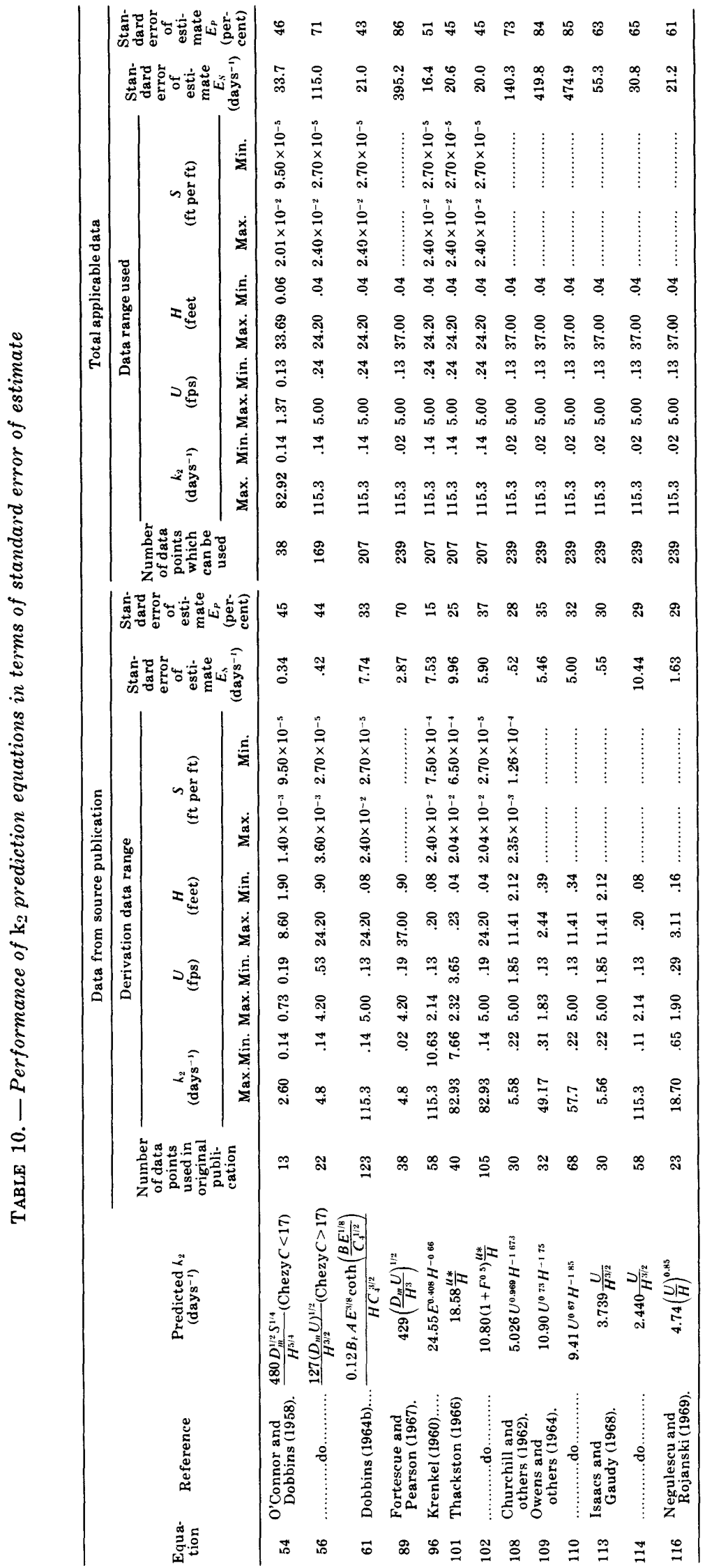




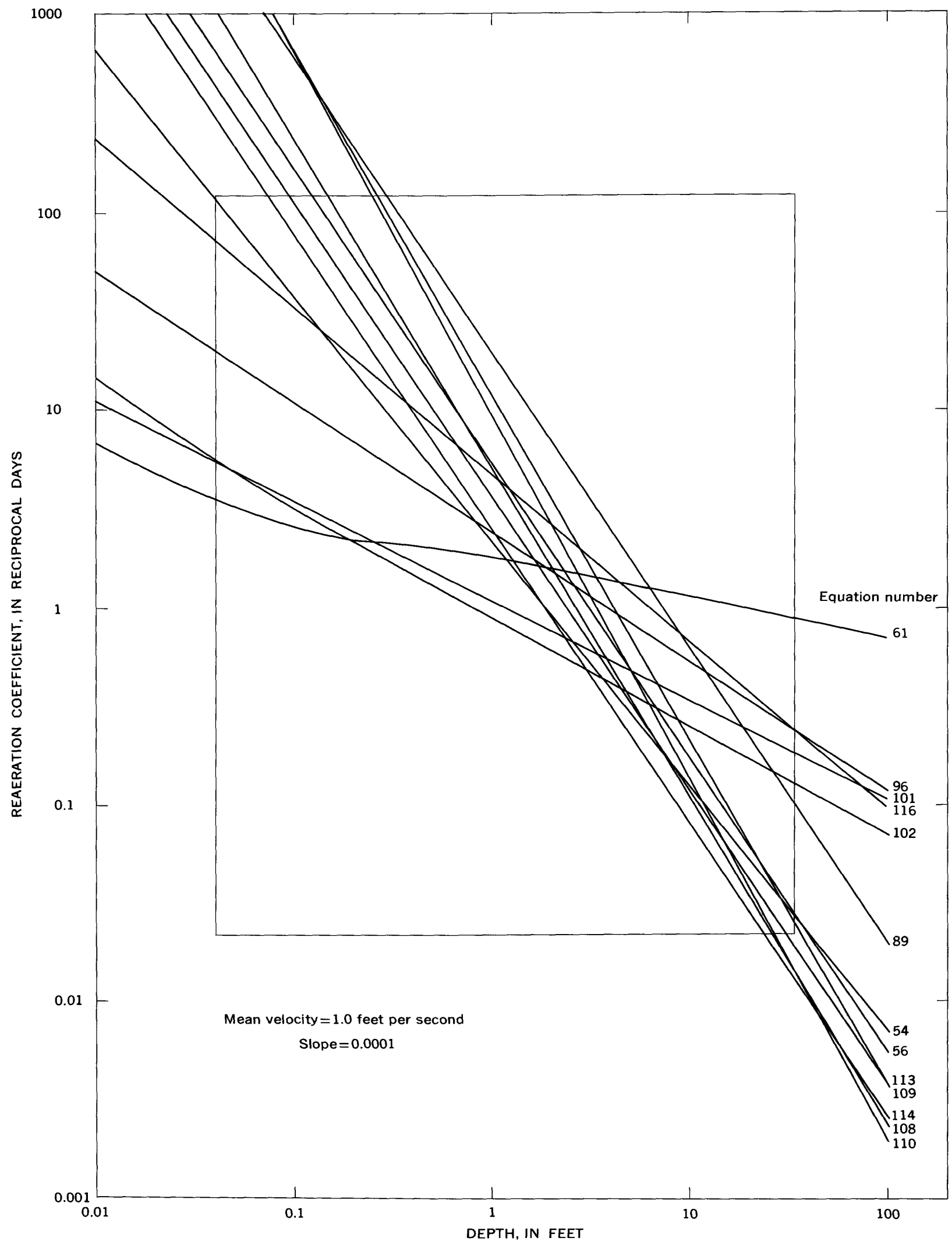

FIGURE 17. - Reaeration coefficient as a function of depth, from prediction equations in table 10. 


\section{REGRESSION ANALYSIS}

In general, regression analysis consists of computing the coefficients for an assumed equation form so that the sum of the squares of the departures of the experimental data points from the curve given by the equation is a minimum. For example, if for the data set $Y_{i}=G\left(X_{1 i}, X_{2 i}, \ldots, X_{n i}\right)$, the linear model

$$
Y_{e i}=A_{0}+A_{1} X_{1 i}+A_{2} X_{2 i}+\ldots+A_{n} X_{n i}
$$

is used to obtain estimates $Y_{e}$ of the dependent variable $Y$, then the $A_{i}, i=0, \ldots n$, are chosen so that the quantity

$$
N^{\prime} E_{S}{ }^{2}=\sum_{i=1}^{N^{\prime}}\left[Y_{e i}-Y_{i}\right]^{2}
$$

is minimized. In equation $158, N^{\prime}$ is the number of data sets used.

The best measure of the goodness of fit of a regression equation to a particular data group is the standard error of estimate $E_{S}$, as defined by equation 154. A parameter which can be used to determine the relative significance of a particular variable to a given regression equation is the partial correlation coefficient. The partial correlation coefficient is the correlation coefficient between the dependent variable and the chosen independent variable, all other independent variables being held constant. Its value ranges between -1 and +1 , and the larger its magnitude, the more significant is the chosen variable in the regression equation. The standard error of estimate and the partial correlation coefficient are discussed at much greater length in standard statistical-methods texts such as that by Snedecor and Cochran (1967).

The significance of a particular regression coefficient, $A_{i}$, may be tested using the fact that the distribution of the quantity

$$
R_{i}=\frac{\left(A_{i}-B_{i}\right)}{S_{b i}}
$$

is Student-t distribution with $N^{\prime}-n$ degrees of freedom. In equation $159, B_{i}$ is the population regression coefficient, and $S_{b i}$ is the standard error of the regression coefficient $A_{i}$. (See Snedecor and Cochran (1967) for more detail.)

In conducting a regression analysis, the limitations of the method must be remembered. First, the model to which the data are fit is preselected so that there is freedom only to adjust the coefficients in the model. If the model is incomplete, a particular variable may appear to be of negligible significance, when in reality it may be of great significance. On the other hand, even though the model is incomplete, prediction equations might be obtained which are entirely satisfactory within the range of data used in deriving them. These equations could, however, yield highly erroneous predictions if applied outside the data range. Second, even though the model is correct, the prediction equation will be incorrect if the data are incorrect or incomplete. Finally, in a regression analysis two types of significance must be considered: physical and statistical. For example, a variable may be highly significant in the physical process yet have little significance in the regression equation because the range of this particular variable was not wide enough in the data group chosen. Furthermore, it is possible to have a high statistical significance in the regression equation and yet to have assumed the wrong physical relation for that particular variable in the model. For these reasons, it is best before beginning a regression analysis to have a reliable physical model available. Unfortunately, as will be emphasized later, there are no such models presently available for the reaeration coefficient in terms of the mean values of the flow parameters which will accurately predict reaeration coefficients for the entire range of available data.

In this report, most of the regression analyses were performed using a general linear model and the logarithms of individual variables. The general model assumed was

$$
\begin{aligned}
\log _{10} k_{2}=\log _{10} A_{0}+A_{1} \log _{10} H+A_{2} \log _{10} U & +A_{3} \log _{10} W+A_{4} \log _{10} S,
\end{aligned}
$$

which is more commonly expressed as

$$
k_{2}=A_{0} H^{A_{1}} U^{A_{2}} W^{A_{3}} S^{A_{4}} \text {. }
$$

In several cases, one or more of the $A_{i}$ were assumed to be zero, and the effect on the standard error of estimate of the prediction equation was investigated. The coefficients were computed using the regression program BMD03R of the University of California, Los Angeles, Health Sciences Computing Facility (Dixon, 1965).

The results of a linear least-squares regression analysis on an equation of the form of 160 are generally slightly different from the results of a least-squares regression analysis of an equation of the form of equation 161 , because the distribution of the variance of the logarithms of $k_{2}$ from equation 160 will be different from the distribution of the variance of $k_{2}$ from equation 161 . If the analysis is conducted on an equation of the form of 160 and the result is converted to the form of equation 161, the average width of the scatter band about the prediction equation will increase directly in proportion with $k_{2}$, whereas if the analysis is conducted directly using equation 161, the scatter 
band width will be a constant, independent of $k_{2}$. In this study, it was decided to use an equation of the type of 160 and to accept deviation from the pure least-squares regression analysis procedures because of the relative simplicity of the analytical procedures involved in this type of analysis as compared with a least-squares analysis based on equation 161 .

A prediction equation of the type of equation 161 was chosen for comparison primarily because this type of $k_{2}$ prediction equation has been used frequently in the past. Furthermore, most of the conceptual models available can be reduced, at least for natural streams, to this type of equation. A number of equations of different forms from equation 161 were tried with negligible success in reducing the standard error of estimate.

\section{RESULTS OF REGRESSION ANALYSIS}

The available experimental data may be divided into two general groups: first, those from natural streams, and second, those from laboratory flumes. Basic data groups used in the analysis were the data sets for which all of the variables $k_{2}, H, U, W$, and $S$ were available. These consisted of the 62 field data sets from Churchill, Elmore, and Buckingham (1962) and Owens, Edwards, and Gibbs (1964) and the 110 flume data sets from Krenkel (1960) and Thackston (1966). Regression analyses were performed separately and jointly on the two groups of data. Partial results of these regression analyses are listed in table 11.

Table 11 lists several comparable equations obtained from regression analyses of the field and flume data. The flow depth, $H$, and hydraulic radius, $R_{H}$, were used in the field and flume cases, respectively, because in each case this particular variable resulted in a slightly lower standard error of estimate. To show the importance of each of the variables in the various prediction equations, the partial correlation coefficients are given in table 11. The standard error of estimate has been listed both in terms of logarithms and in terms of percentage of the predicted $k_{2}$ value.

From an analysis of the results presented in table 11, it can be shown that all the prediction equations obtained from the flume data sets are significantly different statistically from the corresponding prediction equations obtained for the field data sets. ("Correspondence" means that the two equations contain the same independent variables.) For example, on comparison of the exponents in the first equation in column nine with those in the second equation in column one, for the variables $U, W, R_{H}$, and $S$, the Student- $t$

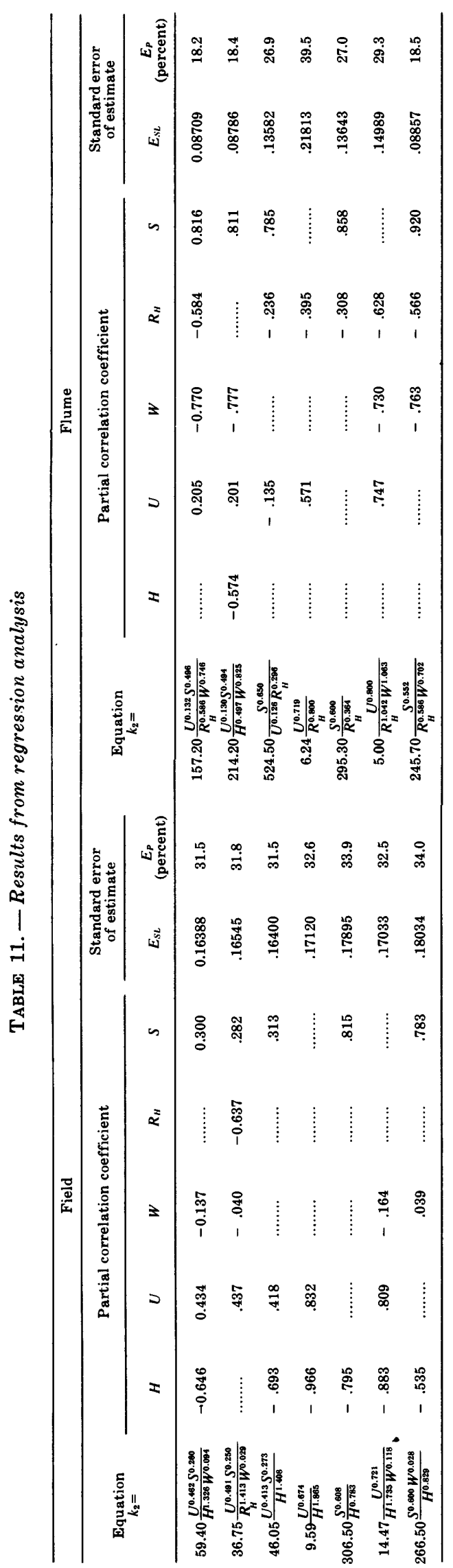


values (see eq 159) are $-5.70,-11.88,10.42$, and 7.18 . For 106 degrees of freedom, these values indicate that the exponents in the two equations are different at a significance level less than 0.005 . For the fifth equation in column nine and its counterpart for the field case $\left(k_{2}=317.2 \frac{S^{0.624}}{R_{H}{ }^{0.750}}\right)$, the Student- $t$ values on $S$ and $R_{H}$ are -0.69 and 3.56. Thus, the exponents on $R_{H}$ are significantly different at some level less than 0.005 , while those on $S$ are different at the 0.25 level. Similar results are obtained for the other equation forms listed. It must be concluded that all the $k_{2}$ prediction equations obtained from flume data are significantly different statistically from those obtained from field data.

The difference between the regression equations from the two groups of data is further illustrated in the standard error of estimate. For example, omitting $W$ from the first equation of column nine, table 11, causes an increase in the standard error of estimate of 8.7 percent, but there is no noticeable effect when this parameter is omitted from the first equation in column one. Furthermore, the largest standard error of estimate occurs for the field equations when the mean velocity, $U$, is omitted, and for the flume equations when the slope, $S$, is omitted.

Finally, the partial correlation coefficients listed in table 11 also indicate a difference between field and flume prediction equations. The field equations have partial correlation coefficients decreasing in the order of $H, U, S$, and $W$, with the coefficient for $W$ negligible. On the other hand, the flume equations have partial correlation coefficients decreasing in the order of $S, W, R_{H}$, and $U$.

If a regression analysis is performed on all of the data used in preparing table 11 , the resulting prediction equation is

$$
k_{2}=721 \frac{S^{0.681}}{U^{0.064} W^{0.183} R_{H}^{0.268}},
$$

which has a standard error of estimate of logarithms of $k_{2}$ of 0.15572 , or 30.1 percent. Results of Student- $t$ tests comparing the exponents of the variables in equation 162 with the exponents in the two corresponding equations of table 11 are given in table 12 . From table 12 , it must be concluded that equation 162 is significantly different at some level less than the 0.005 level from the corresponding flume and field $k_{2}$ prediction equations of table 11. Not only are the flume and field prediction equations significantly different from each other, but also the equation using the combined data is different from both of them.
TABLE 12. - Values of Student-t for comparison of the exponents in equation 162 with those for the two corresponding field and fume equations of table 11

\begin{tabular}{crr}
\hline Variable & \multicolumn{2}{c}{ Student-t values } \\
\cline { 2 - 3 } & \multicolumn{1}{c}{ Field } & \multicolumn{1}{c}{ Flume } \\
\hline$U$ & -11.49 & 4.06 \\
$W$ & -3.04 & 11.12 \\
$R_{\mathrm{H}}$ & 16.50 & 4.58 \\
$S$ & 12.00 & 5.15 \\
\hline
\end{tabular}

Discrepancies similar to those pointed out here between field and flume $k_{2}$ prediction equations have been observed in other areas of hydraulic engineering research. For example, in the field of alluvial boundary hydraulies, Maddock (1969, p. A67) concluded:

The variability of natural channels cannot be studied in flumes because of constraints placed upon the possible combinations of width, depth, velocity, slope, and load that are inherent in field operations.

And Colby (1964, p. A45) stated:

Flume investigations can provide much helpful information on sediment transportation; but, until scale effects are understood more completely, flume investigations of the discharge of sands are not model studies of the discharge of sands in field streams.

As another example of the apparent difference between field and flume phenomena, the present authors attempted to relate to the form of $k_{2}$ regression equations the form of the regression equations for the turbulent intensity at one-tenth the flow depth below the water surface. The turbulent intensity, $u^{\prime}$, is the root-mean-square of the deviations of the velocity fluctuations at a point around the mean value of the velocity at the point. It is equal to the square root of twice the kinetic energy per unit of mass of the fluid turbulence at the point, and thus the near-surface value of $u^{\prime}$ might reasonably be expected to be related to the reaeration coefficient.

The results of the regression analysis of data obtained from R. S. McQuivey (written commun., 1970) are listed in table 13. A general model analogous to equation 161 was assumed for the turbulent intensity. Because simultaneous $k_{2}$ and $u$ measurements are not available, no conclusions could be made about the functional relation, if any, between $k_{2}$ and $u^{\prime}$.

On the other hand, in terms of the dependence of $u^{\prime}$ on $S$ and $U$, the results of this study are similar to the results of the reaeration study in that the flume prediction equations show the highest partial correlation with $S$ whereas the field equations show the highest partial correlation with $U$. However, the results of this study differ from the results of the reaeration study in that the dependency of $u^{\prime}$ on $W$ is significant both in the flume and in the field. The results of the Student- $t$ 
TABLE 13. - Dependence of turbulent intensity on the mean hydraulic parameters

\begin{tabular}{|c|c|c|c|c|c|c|c|}
\hline \multirow{2}{*}{$\underset{u^{\prime}=}{\text { Equation }}$} & \multicolumn{4}{|c|}{ Partial correlation coefficients } & \multirow{2}{*}{$E_{S L}$} & \multirow{2}{*}{$\begin{array}{l}\text { Number } \\
\text { of data } \\
\text { points }\end{array}$} & \multirow{2}{*}{ Type of data } \\
\hline & $H$ & $U$ & $W$ & $S$ & & & \\
\hline $0.667 \frac{U^{0.121} W^{0.439} S^{0.390}}{H^{0.068}}$ & -0.045 & 0.085 & 0.302 & 0.560 & 0.115 & 20 & Flume, rigid. \\
\hline $3.161 \frac{H^{0.306} S^{0.503}}{U^{0.090}}$ & .779 & -.068 & $\cdots \cdots$ & .700 & .117 & 20 & Do. \\
\hline $1.303 \frac{U^{0.143} W^{0.798} S^{0.544}}{H^{0.541}}$ & -.390 & .121 & .527 & .590 & .146 & 27 & Flume, alluvial. \\
\hline $0.890 H^{0.158} U^{0.422} S^{0.313}$ & .219 & .315 & $\ldots .$. & .381 & .168 & 27 & Do. \\
\hline $23.750 H^{0.048} U^{0.764} W^{0.757} S^{1.183}$ & .234 & .718 & .490 & .516 & .087 & 43 & Field. \\
\hline $0.121 H^{0.042} U^{1.050} S^{0.090}$ & .176 & .871 & $\ldots$. & .271 & .098 & 43 & Do. \\
\hline
\end{tabular}

tests comparing the exponents on the variables in the two flume situations with those of the field situation of table 13 are listed in table 14. These Student- $t$ values show that at least one exponent in each flume equation differs from the corresponding exponent in the field equation at significance levels less than 0.005 . It must be concluded that the flume regression equations for the turbulent intensity at one-tenth the flow depth below the water surface are significantly different from the field equation.

TABLE 14. - Results of the Student-t test of the exponents in the fume equations and in the field equations of table 13

\begin{tabular}{|c|c|c|c|c|}
\hline \multirow[b]{2}{*}{ Equation } & \multicolumn{4}{|c|}{ Student- $t$ values } \\
\hline & $\boldsymbol{H}$ & $U$ & $\bar{W}$ & $S$ \\
\hline Flume, rigid..... & -0.30 & -1.75 & -0.89 & -5.16 \\
\hline $\begin{array}{l}\text { Do } \\
\text { Flume, alluvial............ }\end{array}$ & $\begin{array}{r}.79 \\
-2.13\end{array}$ & $\begin{array}{l}-2.33 \\
-2.50\end{array}$ & $\overline{.15}$ & $\begin{array}{r}1.54 \\
-4.02\end{array}$ \\
\hline Do & .79 & -2.33 & - & 1.54 \\
\hline
\end{tabular}

As a final example of the difference between regression equations for flume and field data, consider equations for predicting mean velocity from $W, H$, and $S$ obtained from the same data as the $k_{2}$ prediction equations of table 11 . These equations along with the partial correlation coefficients and the standard errors of estimate are listed in table 15 .

TABLE 15. - Prediction equations for mean velocity, obtained from the data used in table 11

\begin{tabular}{|c|c|c|c|c|c|c|}
\hline \multirow[t]{2}{*}{$\underset{U=}{\text { Equation }}$} & \multicolumn{3}{|c|}{$\begin{array}{c}\text { Partial } \\
\text { correlation } \\
\text { coefficients }\end{array}$} & \multirow[t]{2}{*}{$E_{S L}$} & \multirow[t]{2}{*}{$\underset{\text { (percent) }}{E_{P}}$} & \multirow{2}{*}{$\begin{array}{l}\text { Type } \\
\text { of } \\
\text { data }\end{array}$} \\
\hline & $\boldsymbol{W}$ & $\boldsymbol{R}_{H}$ & $S$ & & & \\
\hline $37.55 W^{0.187} R_{H}^{1.168} S^{0.742}$ & 0.256 & 0.676 & 0.861 & 0.166 & 31.8 & Field. \\
\hline $98.80 R_{H}^{1.474} S^{0.784}$ & $\ldots$. & .937 & .891 & .170 & 32.4 & Do. \\
\hline $29.82 W^{0.335} R_{H}{ }^{0.608} S^{0.423}$ & .340 & .468 & .755 & .138 & 27.3 & Flume. \\
\hline $25.20 R_{f}^{p .540} S^{0.400}$ & $\ldots .$. & .409 & .721 & .146 & 28.6 & Do. \\
\hline
\end{tabular}

The partial correlation coefficients and the small change in standard error of estimate on omitting $W$ from the equations indicate considerably less dependency of $U$ than $k_{2}$ on this parameter. The partial correlation coefficients further indicate that $U$ in the flume is less dependent on depth than in the field. The difference between the two situations is, however, best illustrated by the Student- $t$ test. Comparing the exponents of the third equation with those of the first in column one of table 15 , the Student- $t$ values for $W, R_{H}$, and $S$ are 1.65 , -5.02 , and -9.94 , whereas in the second and fourth equations, for $R_{H}$ and $S$, the Student- $t$ values are -8.05 and -10.58 . This indicates that the prediction equations for the two situations are different at some significance level less than 0.005 .

Possible reasons for the difference between the regression equations for flume and field reaeration coefficient data are (1) differences in the basic physical processes, or misinterpretation of them, (2) statistical quirks, and (3) deficiencies or bias in the available data.

Possible physical differences between the two systems are easy to visualize. For example, the straight, uniform flume flow may have entirely different turbulent transfer properties than the tortuous, nonuniform field stream flows. R. S. McQuivey (written commun., 1970) indicated that the turbulence profile in a 200 -foot-long by 8 -footwide flume at depths of about 1 foot does not fully develop at any point in the flume. This indicates some question about the similarity between the turbulent transfer properties near the surface of field and flume flows. In addition, the high partial correlation coefficient between $k_{2}$ and $W$ in the flume as compared with $u^{\prime}$ and $U$ indicates a possible secondary circulation effect on $k_{2}$ which is not present in the other phenomena. If such an effect is present, simple $W / H$ scaling may not account for it. As a further example, the comments of Maddock (1969) and Colby (1964) quoted previously could be applied equally as well to reaeration as to sediment transport. That is, the different constraints on the two physical systems might prevent similar mass-transfer regimes from developing at the water surface. Finally, the difference 
between the two sets of equations may be due to a basic misunderstanding of the turbulent-masstransfer process near the surface - that is, it may be due to a lack of knowledge of the pertinent scaling parameters, or to the fact that the correct parameters have not been measured.

Certain quirks are inherent in regression analysis which might cause field and flume regression equations for $k_{2}$ to differ. In the first place, the differences between the two systems cause the experimental variables to be selected differentlythat is, in the flume, slope and discharge vary and determine velocity, whereas in the field, depth and discharge vary to determine velocity. This difference in selection of "independent" variables for analysis can be reflected in the regression equations, as can the differences in the adjustments of the so-called independent variables to each other. In the second place, if a wide enough range of data is not available for each of the independent variables, the significance of the variable may be missed in the regression analysis, or the variable may be represented incorrectly in the regression equation. In this sense, dependency of the flume equations on $W$ is particularly questionable because only two values were available in the literature.

Secondly, if the form of the regression equation chosen is incorrect, the equations for the two systems of different scale might be expected to differ radically. This is, in a different sense, a statement of the scaling problem mentioned above.

Finally, no regression equation is better than the data used in its derivation, because if the data contain errors or bias, this will be reflected in the prediction equations. In the field data, especially, the accuracy of the data can be questioned. First, because in none of the studies reported, except possibly that of Churchill, Elmore, and Buckingham (1962), have sedimentation, bottom deposits, and photosynthesis been accounted for adequately. Second, in the two sets of flume data, there is a possibility that one or both of the data sets were influenced by an oxygen-consuming reaction in the flume channel, and this would have a tendency to bias the resulting data. (See appendix A.)

In addition to the differences pointed out above between regression equations derived from different data sets, it can also be shown that the regression equations (listed in table 11) for the field data are significantly different from most comparable equations found in the literature.

The Student- $t$ test was used to compare exponents on various variables of the equations in table 11 with the corresponding exponents from the equations in the literature, and the results are presented in table 16. The Student- $t$ values show that all but the two equations of Owens, Edwards, and Gibbs (1964) have at least one exponent which differs from the exponent in the corresponding field equation at a level less than the 0.005 significance level. Because the second equation of Owens, Edwards, and Gibbs (1964) was derived, with the exception of the six data sets of Gameson, Truesdale, and Downing (1955), from the same data as the corresponding field regression equation, it is not surprising that these equations do not differ significantly.

The previous discussion suggests at least two reasons for pursuing the search for a new physical model describing reaeration. The first is the difference observed between the regression equations for the field and flume situations. It would seem that equations describing the physical process of

TABLE 16. - Results of the Student-t test of exponents in the regression equations of this study and corresponding equations in the literature

\begin{tabular}{|c|c|c|c|c|c|c|}
\hline \multirow{2}{*}{$\begin{array}{c}\text { Regression equations } \\
\text { (this study) } \\
k_{2}=\end{array}$} & \multirow{2}{*}{$\begin{array}{l}\text { Comparable equations } \\
\text { in the literature } \\
k_{2}=\end{array}$} & \multicolumn{3}{|c|}{ Student- $t_{0.005}$} & \multirow{2}{*}{$\underset{\text { (percent) }}{E_{P}}$} & \multirow{2}{*}{ Source } \\
\hline & & $H$ & $U$ & $S$ & & \\
\hline $46.05 \frac{U^{0.413} S^{0.273}}{H^{1.408}}$ & $24.55(U S g)^{0.408} H^{-0.68} \quad\left(T=20^{\circ} \mathrm{C}\right)$ & -3.89 & 0.04 & -1.24 & 31.5 & Krenkel (1960). \\
\hline \multirow{5}{*}{$9.59 \frac{U^{0.674}}{H^{1.865}}$} & $127 D_{m}^{1 / 2}\left(\frac{U}{H^{3}}\right)^{1 / 2} \quad($ Chezy $C>17)$ & -5.60 & 2.98 & ......... & 32.6 & $\begin{array}{l}\text { O'Connor and } \\
\text { Dobbins (1958). }\end{array}$ \\
\hline & $5.026 U^{0.969} H^{-1673} \quad\left(T=20^{\circ} \mathrm{C}\right)$ & -2.95 & -5.04 & $\ldots \ldots$ & $\ldots \ldots \ldots$ & $\begin{array}{l}\text { Churchill and } \\
\text { others (1962). }\end{array}$ \\
\hline & $10.90 U^{0.73} \mathrm{H}^{-1.75}$ & -1.77 & -.96 & ........ & 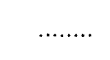 & $\begin{array}{l}\text { Owens and } \\
\text { others (1964). }\end{array}$ \\
\hline & $9.41 U^{0.67} H^{-185}$ & -.23 & -.07 & $\ldots \ldots \ldots$ & $\ldots \ldots .$. & Do. \\
\hline & $4.74\left(\frac{U}{H}\right)^{0.85}$ & -15.58 & -3.01 & ........ & $\ldots \ldots \ldots$ & $\begin{array}{l}\text { Negulescu and } \\
\text { Rojanski (1969). }\end{array}$ \\
\hline \multirow{2}{*}{$306.50 \frac{S^{0608}}{H^{0783}}$} & $4.80 D_{m}^{1 / 2}\left(\frac{S}{H^{5}}\right)^{1 / 4} \quad($ Chezy $C<17)$ & -6.00 & $\ldots \ldots \ldots$ & 6.35 & 33.9 & $\begin{array}{l}\text { O'Connor and } \\
\text { Dobbins (1958). }\end{array}$ \\
\hline & $18.58 \sqrt{g}\left(\frac{S}{H}\right)^{1 / 2}$ & -3.64 & $\ldots \ldots \ldots$ & 1.92 & 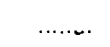 & Thackston (1966). \\
\hline
\end{tabular}

$\nmid$ Equations by Fortescue and Pearson (1967) and Isaacs and Gaudy (1968) are also of this form. 
reaeration should be applicable no matter what the scale of the system. The second is the fact that none of the available theoretical models can be related to the regression equation for field data. This is because either the theoretical model is not expressed in terms of the mean hydraulic parameters, or because for the ones which are expressed in this fashion, it has been shown that the exponents in the field regression equation differ significantly from those in the theoretical models. Furthermore, the commonly measured mean hydraulic parameters $U, H, W$, and $S$ may not be sufficient to model the phenomenon. Perhaps some other parameters must also be measured, such as those characteristic of the near-surface turbulence, or of the velocity in the air above the flow.

Using all the available data and using the standard error of estimate of logarithms as the basis for judgment, one can see from table 11 that the best prediction equation for reaeration coefficients of natural streams is

$$
k_{2}=46.05 \frac{U^{0.413} S^{0.273}}{H^{1.408}} .
$$

However, considering the percentage variation (table 11), the equation

$$
k_{2}=9.59 \frac{U^{0.674}}{H^{1.865}}
$$

has a percentage variation only 1.1 percent greater than that for equation 163 . Considering the difficulty and expense of measuring accurately the slope of a field stream (Yao, 1969), an equation that does not contain the slope is preferred for routine use as a prediction equation for $k_{2}$ in natural streams.

In appendix $B$, in addition to the field data used in deriving equation 164, there are other field data which report only $U$ and $H$. From the 121 sets of field data which contain $U$ and $H$, the least-squares regression equation for $k_{2}$ is

$$
k_{2}=8.76 \frac{U^{0.607}}{H^{1.689}}
$$

Equation 165 has a standard error of estimate of logs of 0.203 (37.5 percent). The Student- $t$ test was used to compare the exponents on $U$ and $H$; comparing equation 165 with equation 164 (119 degrees of freedom) for $U$ and $H$, the Student- $t$ values are -1.42 and 4.22 , whereas comparing equation 164 and 165 (60 degrees of freedom) gives Student- $t$ values of 1.14 and -2.70 . Thus equations 164 and 165 have one exponent which differs from its counterpart at a significance level less than 0.005 . The standard error of estimate of equation 165 has increased markedly over that of 164 . This is probably due to a decrease in overall quality of the data. However, because equation 165 is based on the widest range of field data presently available, this equation is probably the best available for prediction of reaeration coefficients for natural streams.

\section{EFFECT OF DETERGENTS AND WIND ON THE REAERATION COEFFICIENT}

Up to this point, the reaeration process has been considered as occurring under ideal conditions that is, in relatively pure water with no wind effects. The literature, however, provides evidence that the presence of sewage and surfactants in the water tends to reduce $K_{L}$, the liquid-film coefficient for gas transfer, compared with the pure-water value, whereas winds tend to increase $K_{L}$ with respect to the value existing for quiescent air conditions. Recall that $K_{L}=K_{2} H$, where $K_{2}$ is the reaeration coefficient and $H$ is the mean depth of flow. The results of some of these studies are summarized in the following paragraphs.

Mancy and Okun (1965) discussed two theories concerning the effect of surfactants on the rate of oxygen absorption. On the one hand, they cited four references suggesting that surfactants reduce the absorption rate only by decreasing the hydrodynamic activity at the interface. On the other hand, they cited six references suggesting that surfactants reduce the absorption rate both by increasing the diffusional resistance at the interface and by decreasing the hydrodynamic activity at the interface.

Eckenfelder, Raymond, and Lauria (1956) hypothesized that surfactants would reduce $K_{L}$ in two ways: first, by increasing the diffusional resistance through a reduction in the surface tension, and second, by decreasing the surface renewal rate through an increase in viscosity. Metzger (1968) studied the absorption of helium and nitrogen under identical mixing conditions in a stirred tank containing $0.5 \mathrm{mg} / \mathrm{l}$ of ABS (alkyl benzene sulfonate), a surfactant. He found that the film thickness and surface-renewal parameters of the Dobbins (1956) film-penetration model were larger and smaller, respectively, than the values obtained for the same mixing conditions with distilled water. Downing, Melbourne, and Bruce (1957) studied the effects of household detergents and sewage on oxygen absorption in a stirred tank and in a wave tank. They found that mixed household detergents reduced $K_{L}$ from the clean-water value by various amounts depending on the concentration of detergent, the initial $K_{L}$ value, and the way in which the water was agitated. The effects of sewage effluents and settled sewage containing detergents were similar 
to the effects observed for mixed detergents, but the effects of sewage effluents and settled sewage were less when the detergents were not present.

Gameson, Truesdale, and Varley (1956) studied the effect of about $1 \mathrm{mg} / \mathrm{l}$ ABS on oxygen absorption by flowing water in a laboratory open channel. They found that the percentage reduction in $K_{L}$ depended on the degree of turbulence, expressed in terms of $K_{L}$. The maximum reduction occurred for intermediate $K_{L}$ values, equivalent to those of lowland English streams. Lower and higher turbulence conditions resulted in smaller percentage reductions. Similarly, Metzger (1968) and Mancy and Okun (1965) found in stirred-tank studies that the largest reduction in $K_{L}$ occurred for intermediate mixing intensities.

Gameson, Truesdale, and Downing (1955) added detergents to a small English stream and found that there was no apparent relation between the reduction in $K_{L}$ and the detergent concentration, in the range from 0.5 to $2 \mathrm{mg} / \mathrm{l}$. The reduction in $K_{L}$ decreased with increasing $K_{L}$ for values ranging from about 30 to $120 \mathrm{~cm} / \mathrm{hr}$ (centimeters per hour). No maximum was observed in the natural streams, apparently because $K_{L}$ values were all larger than 5 to $10 \mathrm{~cm} / \mathrm{hr}$, the level at which the maximum reduction occurred in the laboratory studies.

Ogden, Gibbs, and Gameson (1959) studied the effect of salinity and the combined effect of salinity, detergents, and sewage on the reaeration rate of flowing water in a flume. They found that the percentage reduction in $K_{L}$ was equal to six times the square root of the salinity expressed as grams of salt per 1,000 grams of mixture, but that the reductions caused by detergents and sewage did not appear to depend on the presence of salts in the water. Kehr (1938) studied the effect of sewage on reaeration in a laboratory flume and found that the percentage reduction increased with the percentage of sewage and the flow velocity; however, the maximum velocity considered was only about 0.5 fps.

Lynch and Sawyer (1954) studied the effect on oxygen absorption of 11 synthetic detergents available on the market in 1954. They found with one exception that all reduced the oxygen-transfer coefficient, and they pointed out that because these detergents were not biodegradable, they eventually entered the streams and rivers, where they could significantly reduce the reaeration coefficients. However, since 1954, biodegradable detergents have been developed, and these should reduce the effect that detergents have on reaeration coefficients.
Downing and Truesdale (1955) studied the effect of oil films on oxygen absorption in a stirred tank. They found no significant effect until the film thickness exceeded $10^{-4} \mathrm{~cm}$. Thus, they concluded that oil films were not a problem because films thicker than $10^{-4} \mathrm{~cm}$ were not likely to persist very long in natural streams.

Poon and Campbell (1967) found that the presence of small concentrations of biologically inert suspended particles increased the rate of oxygen absorption in an aerator. This finding is in agreement with the observation of R. S. McQuivey (oral commun., 1970) that suspended sediment increases the intensity of turbulence over that observed in a clear water flow.

Whereas surfactants and sewage generally reduce $K_{L}$, the presence of winds and surface disturbances produced by the winds generally increase $K_{L}$. Imhoff and Fair (1956, p. 291) discussed wind effects in general terms. They suggested that winds passing over the water surface may double the absorption rate, that wind-induced waves may increase the absorption rate 10 times, and that whitecaps may, by droplet aeration, increase it 100 times.

Eloubaidy (1969) studied reaeration both with and without wind waves in a laboratory wind-water tunnel. He concluded that the absorption rate was significantly increased when waves developed on the water surface, and this increase was considerably more than could be accounted for by the increase in surface area. He presented a prediction equation containing the shear velocity, the kinematic viscosity, and the wind velocity. This equation, however, was verified only with a very limited amount of data.

Downing and Truesdale (1955) studied the effect of surface wind on oxygen absorption in a laboratory ripple tank. They found that there was little change in $K_{L}$ until a wind velocity between 3.0 and 3.5 meters per second was reached. For larger velocities, $K_{L}$ increased rapidly. Similar results were obtained in tank experiments by Kanwishe: (1963), who found little change in the gas-exchange coefficient up to a velocity of about 3 meters per second but a coefficient that increased approximately as the square of the velocity for wind velocities between 3 and 10 meters per second. Downing and Truesdale (1955) also studied the effect of wave height and frequency on $K_{L}$. They found that the rate of absorption of oxygen increased almost linearly with increase in wave height, increasing approximately fourfold for a fourfold increase in height. For irregular choppy 
waves of the same frequency, $K_{L}$ increased less rapidly. They also found that $K_{L}$ increased with an increase in the frequency of the waves, although there was more scatter in the results. Finally, Juliano (1969) found that absorption-rate constants measured in the Sacramento-San Joaquin estuary system varied directly with wind velocity. There was considerable scatter in the relation; however, this scatter was attributed to the fact that wind effects vary greatly from point to point in the reach of interest.

\section{CONGLUSIONS AND RECOMMENDATIONS}

\section{CONCLUSIONS}

From the review of reaeration in open-channel flow presented in previous sections, we have arrived at several general conclusions.

1 . The approximations that can be made in the general DO balance equation (see pages 22-29, 33-39) vary radically from situation to situation. Therefore, careful consideration of all the circumstances must be made before any terms of the equation are disregarded.

2. The most general and realistic conceptual model for the reaeration process of open-channel flows is the surface-renewal-damped-eddy diffusivity model of King (1966). This model merits further development in relating its parameters to the mean flow parameters that are easily measured.

3. In theory, the tracer technique is far superior to the other two techniques commonly used for determining the reaeration coefficient of openchannel flows. In practice, however, the use of radioactive tracers involves problems from the standpoint of obtaining permission for use in natural streams, and from the standpoint of possible hazard to the personnel involved in the study.

4. Sensitivity analysis of the DO balance technique shows that if photosynthetic-oxygen production is present, its neglect is probably the most serious source of error in the calculation of the reaeration coefficient. Following this in decreasing order of effect are errors in the upstream and downstream DO deficit, $k_{1}$, and $L_{\mathrm{a}}$.

5. Sensitivity analysis of the disturbed-equilibrium technique shows that the most serious sources of error in the calculation of the reaeration coefficient are, in decreasing order of effect on the calculated $k_{2}$, errors in the downstream DO deficit, net photosynthetic production, upstream DO deficit, and time of travel.
6. The expected root-mean-square error of 15 percent for the tracer technique is much less than the 65-percent error expected from the DO balance technique and the 115-percent error expected from the disturbed-equilibrium technique. Thus, it would seem well worthwhile to expend further time and effort on developing the tracer technique for use in streams of all sizes.

7. The best available reaeration-coefficient data for natural streams is that of Churchill, Elmore, and Buckingham (1962). With these data and those of Owens, Edwards, and Gibbs (1964), 62 field data sets can be considered complete; that is, they contain all the mean hydraulic parameters commonly measured: velocity, depth, width, and slope.

8. Analysis of available reaeration-coefficient data from natural streams and from laboratory flumes indicates that there is a significant difference between the $k_{2}$ regression equations obtained from the two groups of data. The most likely reasons for this are (a) an inability to scale correctly the turbulent mass-transfer process at the water surface, (b) statistical quirks involved in regression analysis using the complexly interdependent hydraulic parameters of mean velocity, depth, width, and slope, and (c) experimental bias in one or more of the individual groups of data.

9. For the prediction of $k_{2}$ in natural streams, regression analysis indicates that the equation with the smallest standard error of estimate consistent with the ease of obtaining the mean flow parameters is $k_{2}=8.76 \frac{U^{0.607}}{H^{1.689}}$, where $k_{2}$ is in reciprocal days.

\section{RECOMMENDATIONS}

A more detailed and reliable theoretical model than is presently available for the transfer of oxygen through the surface of an open-channel flow is needed. The model should account for the effect of impurities in the flow such as sewage, sediment, dissolved solids, and surfactants. It should consider the effect of wind blowing over the water surface, and it should be related directly to hydraulic parameters such as mean velocity, depth, width, slope, and possibly near-surface vertical and (or) longitudinal turbulence. The model should, furthermore, scale the interface masstransfer phenomenon so that field and flume-scale data fit one general equation. This would make possible (with adequate independent verification of the equation) the use of experimental results from laboratory flumes to predict field-scale reaeration coefficients. 
For verification of the model in large-scale field streams in which some degree of pollution, photosynthesis, and other interferences may be present, or for obtaining more reliable $k_{2}$ values than are presently available, the tracer technique should be modified to avoid the use of the radioactive tracers. This could be done, for example, by using the fluorescent dye as the conservative tracer and gas chromatography for determining the concentration of some inert tracer gas.

If the DO balance technique and the disturbedequilibrium technique are to be used for calculating reaeration coefficients, then conclusions 4 and 5 above indicate that better methods must be found for accounting for photosyntheticoxygen production and for the respiration of the suspended and attached aquatic plants. In fact, all the source-sink terms should be more carefully considered in reaeration-coefficient studies than they are presently.

Other factors concerning DO in streams require immediate consideration. For example, consider the lateral distribution of $\mathrm{DO}$ in a stream cross section. The measurements by Churchill, Elmore, and Buckingham (1962) indicate very large DO variations in a stream cross section. The implications of this observation in terms of average DO transferred into the stream and in terms of the cross-section variation of the surface masstransfer coefficient are very important. A further field study in terms of lateral DO distribution might be a study of the effect of sewage outfall placement on dispersion, or concentration of pollution by the stream.

The probabilistic behavior of DO variations at a particular cross section also needs further study. Studies should be aimed at determining the hazard level associated with a particular pollution input at some point upstream of the cross section of interest.

\section{REFERENCES}

Adeney, W. E., and Becker, H. G., 1919, The determination of the rate of solution of atmospheric nitrogen and oxygen by water: Philos. Mag., v. 38, p. 317-338.

1920 , Determination of the rate of solution of atmospheric nitrogen and oxygen by water: Philos. Mag., v. 39 , p. 385-404.

American Public Health Association, 1960, 1965, Standard methods for the examination of water and wastewater: New York, 769 p. (11th ed., 1960; 12th ed., 1965).

Bain, R. C., Jr., 1968, Predicting DO variations caused by algae: Am. Soc. Civil Engineers Jour., v. 94, no. SA-5, p. $867-881$.
Baird, M. H. I., and Davidson, J. F., 1962, Gas absorption, part II of Annular jets: Chem. Eng. Sci., v. 17, p. 473-480.

Baity, H. G., 1938, Some factors affecting the aerobic decomposition of sewage sludge deposits: Sewage Works Jour., v. 10 , no. 3 , p. 539-568.

Bella, D. A., 1969, Discussion of atmospheric oxygenation in a simulated stream: Am. Soc. Civil Engineers Jour., v. 95, no. SA-2, p. 358-362.

Bella, D. A., and Dobbins, W. E., 1968, Difference modeling of stream pollution: Am. Soc. Civil Engineers Jour., v. 94, no. SA-5, p. 995-1016.

Bird, R. B., Stewart, W. E., and Lightfoot, E. N., 1960, Transport phenomena: New York, John Wiley \& Sons, Inc., $780 \mathrm{p}$.

Brown, C. L., and Clark, R., 1968, Observations on dredging and dissolved oxygen in a tidal waterway: Water Resources Research, v. 4, no. 6, p. 1381-1384.

Calderbank, P. H., and Moo-Young, M. B., 1961, The continuous phase heat and mass-transfer properties of dispersions: Chem. Eng. Sci., v. 16, p. 39-54.

Camp, T. R., 1963, Water and its impurities: New York, Reinhold Book Corp., 355 p.

1965, Field estimates of oxygen balance parameters: Am. Soc. Civil Engineers Jour., v. 91, no. SA-5, p. 1-16.

Carslaw, H. S., and Jaeger, J. C., 1959, Conduction of heat in solids: London, Oxford Univ. Press, $510 \mathrm{p}$.

Churchill, M. A., Elmore, H. L., and Buckingham, R. A., 1962, The prediction of stream reaeration rates: Am. Soc. Civil Engineers Jour., v. 88, no. SA-4, p. 1-46.

Colby, B. R., 1964, Discharge of sands and mean-velocity relationships in sand-bed streams: U.S. Geol. Survey Prof. Paper 462-A, 47 p.

Committee on Sanitary Engineering Research, 1960, Solubility of atmospheric oxygen in water: Am. Soc. Civil Engineers Jour., v. 86, no. SA-4, p. 41-53.

Courchaine, R. J., 1968, Significance of nitrification in stream analysis - effects on the oxygen balance: Water Pollution Control Federation Jour., v. 40, no. 5, p. 835-847.

Danckwerts, P. V., 1951, Significance of liquid-film coefficients in gas absorption: Indust. and Eng. Chemistry, v. 43, no. 6, p. 1460-1467.

Davies, J. T., and Rideal, E. K., 1963, Interfacial phenomena [2d ed.]: New York, Academic Press, $474 \mathrm{p}$.

DiToro, D. M., and O'Connor, D. J., 1968, The distribution of dissolved oxygen in a stream with time varying velocity: Water Resources Research, v. 4, no. 3, p. 639-646.

Dixon, W. J., 1965, Biomedical computer programs: Health Sciences Computing Facility, Univ. of California, Los Angeles, $620 \mathrm{p}$.

Dobbins, W. E., 1956, The nature of the oxygen transfer coefficient in aeration systems, in Biological treatment of sewage and industrial wastes: New York, Reinhold Book Corp.; p. 141-253.

1964a, Mechanism of gas absorption by turbulent liquids: Internat. Conf. Water Pollution Research Proc., London, England, Sept. 1962, Pergamon Press, p. 60-76. $1964 \mathrm{~b}, \mathrm{BOD}$ and oxygen relationships in streams: Am. Soc. Civil Engineers Jour., v. 90, no. SA-3, p. 53-78. 1965 , Closure to BOD and oxygen relationships in streams: Am. Soc. Civil Engineers Jour., v. 91, no. SA-5, p. 49-55.

Downing, A. L., Melbourne, K. V., and Bruce, A. M., 1957, The effect of contaminants on the rate of aeration of water: Jour. Appl. Chemistry, v. 7, Nov., p. 590-596. 
Downing, A. L., and Truesdale, G. A., 1955, Some factors affecting the rate of solution of oxygen in water: Jour. Appl. Chemistry, v. 5, Oct., p. 570-581.

Dresnack, Robert, and Dobbins, W. E., 1968, Numerical analysis of BOD and DO profiles: Am. Soc. Civil Engineers Jour., v. 94, no. SA-5, p. 789-807.

Duda, J. L., and Vrentas, J. S., 1968, Laminar liquid jet diffusion studies: Am. Inst. Chem. Engineers Jour., v. 14, no. 2, p. 286-294.

Eckenfelder, W. W., Jr., Raymond, L. W., and Lauria, D. T., 1956, Effect of various organic substances on oxygen absorption efficiency: Water Pollution Control Federation Jour., v. 28, no. 11, p. 1357-1364.

Edwards, R. W., 1962, Some effects of plants and animals on the conditions in fresh-water streams with particular reference to their oxygen balance: Internat. Jour. Air and Water Pollution, v. 6, p. 505-520.

1968, Plants as oxygenators in rivers: Water Research, v. 2, no. 3, p. 243-248.

Edwards, R. W., and Owens, M., 1962, The oxygen balance of a chalk stream, part IV of The effects of plants on river conditions: Jour. Ecology, v. 50, Feb., p. 207-220.

1965, The oxygen balance of streams: Fifth Symposium British Ecol. Soc., Oxford, Blackwell Sci. Pubs., p. 149-172.

Edwards, R. W., Owens, M., and Gibbs, J. W., 1961, Estimates of surface aeration in two streams: Inst. Water Engineers Jour., v. 15, no. 5, p. 395-405.

Edwards, R. W., and Rolley, H. L. J., 1965, Oxygen consumption of river muds: Jour. Ecology, v. 53, Mar., p. 1-19.

Elmore, H. L., 1955, Determination of BOD by a reaeration technique: Water Pollution Control Federation Jour., v. 27, no. 9, p. 993-1002.

Elmore, H. L., and West, W. F., 1961, Effect of water temperature on stream reaeration: Am. Soc. Civil Engineers Jour., v. 87, no. SA-6, p. 59-71.

Eloubaidy, A. F., 1969, Wind waves and the reaeration coefficient in open channel flow: Fort Collins, Colo., Colorado State Univ. Dept. Civil Eng. Ph. D. dissert.

Fair, G. M., 1936, The "log-difference" method of estimating the constants of the first-stage biochemical oxygen demand curve: Sewage Works Jour., v. 8, no. 3, p. 430-434. 1939, The dissolved oxygen sag - an analysis: Sewage Works Jour., v. 11, no. 3, p. 445-461.

Fair, G. M., Moore, E. W., and Thomas, H. A., Jr., 1941, The natural purification of river muds and pollutional sediments: Sewage Works Jour., v. 13, no. 2, p. 270-307.

Fischer, H. B., 1967, The mechanism of dispersion in natural streams: Am. Soc. Civil Engineers Jour., v. 93, no. HY-6, p. 187-216.

Fortescue, G. E., and Pearson, J. R. A., 1967, On gas absorption into a turbulent liquid: Chem. Eng. Sci., v. 22, p. 1163-1176.

Frankel, R. J., 1965, Economic evaluation of water quality, an engineering-economic model for water quality management: Sanitary Eng. Research Lab. Rept. 65-3, Univ. of California, Berkeley, Calif., 167 p.

Gameson, A. L. H., and Truesdale, G. A., 1959, Some oxygen studies in streams: Inst. Water Engineers Jour., v. 13, no. 2 , p. 175-187.

Gameson, A. L. H., Truesdale, G. A., and Downing, A. L., 1955, Reaeration studies in a lakeland beck: Inst. Water Engineers Jour., v. 9, no. 7, p. 571-594.
Gameson, A. L. H., Truesdale, G. A., and Varley, R. A., 1956 , Some factors affecting the aeration of flowing water: Water and Sanitary Eng., v. 6, p. 52.

Gannon, J. J., 1966, River and laboratory BOD rate considerations: Am. Soc. Civil Engineers Jour., v. 92, no. SA-1, p. 135-161.

Gannon, J. J., Pelton, J. R., and Westfield, J., 1965, Respirometer assembly for BOD measurement of river waters and biologically treated effluents: Internat. Jour. Air and Water Pollution, v. 9, p. 27-40.

Gindler, B. J., 1967, Water pollution and quality controls, v. 3 of Water and water rights, Robert E. Clark, ed.: Ind., The Allen Smith Co., 540 p.

Gunnerson, C. G., 1966, Discussion of river and laboratory BOD rate considerations: Am. Soc. Civil Engineers Jour., v. 92 , no. SA-1, p. 135-161.

Gunnerson, C. G., and Bailey, T. E., 1963, Oxygen relationships in the Sacramento River: Am. Soc. Civil Engineers Jour., v. 89, no. SA-4, p. 95-124.

Haney, P. D., 1966, What is pollution? An engineer's viewpoint: Am. Soc. Civil Engineers Jour., v. 92, no. SA-1, p. 109-113.

Harriott, Peter, 1962, A random eddy modification of the penetration theory: Chem. Eng. Sci., v. 17, p. 149-154.

Higbie, Ralph, 1935, The rate of absorption of a pure gas into a still liquid during short periods of exposure: Am. Inst. Chem. Engineers Trans., v. 31, p. 365-390.

Himmelblau, D. M., 1964, Diffusion of dissolved gases in liquids: Chem. Revs., v. 64 , p. 527-550.

Hinze, J. O., 1959, Turbulence: New York, McGraw-Hill Book Co., 586 p.

Hull, C. H. J., 1959, Bibliography on biochemical oxygen demand: Report 5, Low-flow augmentation project, Dept. Sanitary Eng. and Water Research, The Johns Hopkins Univ., 27 p.

1960, Discussion of oxygen balance of an estuary: Am. Soc. Civil Engineers Jour., v. 86, no. SA-6, p. 105120.

1963, Oxygenation of Baltimore harbor by planktonic algae: Water Pollution Control Federation Jour., v. 35, no. 5, p. 587-606.

1966, Discussion of effect of benthic algae on stream dissolved oxygen: Am. Soc. Civil Engineers Jour., v. 92, no. SA-1, p. 306-313.

1969, Discussion of management and measurement of DO in impoundments: Am. Soc. Civil Engineers Jour., v. 95 , no. SA-1, p. 158-178.

Hull, C. H. J., and De Filippi, J. A., 1963, Discussion of the prediction of stream reaeration rates: Am. Soc. Civil Engineers Jour., v. 89, no. SA-2, p. 97-109.

Imhoff, K., and Fair, G. M., 1956, Sewage treatment [2d ed.]: New York, John Wiley \& Sons, Inc., 338 p.

Isaacs, W. P., and Gaudy, A. F., 1968, Atmospheric oxygenation in a simulated stream: Am. Soc. Civil Engineers Jour., v. 94, no. SA-2, p. 319-344.

Juliano, D. W., 1969, Reaeration measurements in an estuary: Am. Soc. Civil Engineers Jour., v. 95, no. SA-6, p. 11651178.

Kalinske, A. A., 1943, The role of turbulence in river hydraulics, in Proc. 2d Hyd. Conf.: Iowa Univ. Studies Eng. Bull. 27, p. 266-279.

Kanwisher, J.' W., 1963, On the exchange of gases between the atmosphere and the sea: Deep-Sea Research, v. 10, p. 195-207. 
Kehr, R. W., 1938, Effect of sewage on atmospheric reaeration rates under stream flow conditions, part IV of Measures of natural oxidation in polluted streams: Sewage Works Jour., v. 10, no. 2, p. 228-240.

King, C. J., 1966, Turbulent liquid phase mass transfer at a free gas-liquid interface: Indust. and Eng. Chemistry Fundamentals, v. 5, no. 1, p. 1-8.

Kishinevsky, M. Kh., 1955, Two approaches to the theoretical analysis of absorption processes: Jour. Appl. Chemistry, U.S.S.R., v. 28 , p. $881-886$.

Kishinevsky, M. Kh., and Serebryansky, V. T., 1956, The mechanism of mass transfer at the gas-liquid interface with vigorous stirring: Jour. Appl. Chemistry, U.S.S.R., v. 29, p. 29-33.

Kolthoff, I. M., and Izutsu, K., 1964, The ratio of the first to the second diffusion current of oxygen at the dropping mercury electrode: Am. Chem. Soc. Jour., v. 86, no. 7, p. 1275-1279.

Kothandaraman, V., and Ewing, B. B., 1969, A probabilistic analysis of dissolved oxygen - biochemical oxygen demand relationship in streams: Water Pollution Control Federation Jour., v. 41, no. 2, p. R73-R90.

Kozinski, A. A., and King, C. J., 1966, The influence of diffusivity on liquid phase mass transfer to the free interface in a stirred vessel: Am. Inst. Chem. Engineers Jour., v. 12, no. 1, p. 109-116.

Krenkel, P. A., 1960, Turbulent diffusion and the kinetics of oxygen absorption: Berkeley, Calif., Univ. of California Dept. Sanitary Eng. Ph. D. dissert.

Krenkel, P. A., and Orlob, G. T., 1963, Turbulent diffusion and the reaeration coefficient: Am. Soc. Civil Engineers Trans., v. 128, p. 293-334.

Kunz, K. S., 1957, Numerical analysis: New York, McGrawHill Book Co., 381 p.

Langbein, W. B., and Durum, W. H., 1967, The aeration capacity of streams: U.S. Geol. Survey Circ. 542, 6 p.

Lee, J. D., 1951, Simplified method for analysis of BOD data - a discussion: Sewage and Indus. Wastes, v. 23, no. 2, p. 164-166.

Leffel, R. E., 1968, Pollution of the Chao Phraya River estuary: Am. Soc. Civil Engineers Jour., v. 94, no. SA-2, p. 295-306.

Leopold, L. B., and Langbein, W. B., 1960, A primer on water: Washington, U.S. Govt. Printing Office, 50 p.

Lewis, W. K., and Whitman, W. G., 1924, Principles of gas absorption: Indus. and Eng. Chemistry, v. 16, no. 12, p. 1215-1220.

Li, Wen-Hsiung, 1962, Unsteady dissolved-oxygen sag in a stream: Am. Soc. Civil Engineers Jour., v. 88, no. SA-3, p. 75-85.

Liebman, J. C., and Lynn, W. R., 1966, The optimum allocation of stream dissolved oxygen: Water Resources Research, v. 2, no. 3, p. 581-591.

Loucks, D. P., and Lynn, W. R., 1966, Probabilistic models for predicting stream quality: Water Resources Research, v. 2 , no. 3 , p. 593-605.

Ludzack, F. J., Moore, W. A., and Ruchoft, C. C., 1953, A comparative study of derivation procedures for BOD velocity constants: Sewage and Indus. Wastes, v. 25, no. 8 , p. 875-881.

Lynch, W. O., 1965, Discussion of BOD and oxygen relationships in streams: Am. Soc. Civil Engineers Jour., v. 91, no. SA-1, p. $82-84$.
Lynch, W. O., and Sawyer, C. N., 1954, Preliminary studies on frothing and oxygen transfer, part I of Physical behavior of synthetic detergents: Sewage and Indus. Wastes, v. 26, no. 11, p. 1193-1201.

McConnell, W. J., and Sigler, W. F., 1959, Chlorophyll and productivity in a mountain river: Limnology and Oceanography, v. 4 , no. 3 , p. 335-351.

McDonnell, A. J., and Hall, S. D., 1969, Effect of environmental factors on benthal oxygen uptake: Water Pollution Control Federation Jour., v. 41, no. 8, p. R353-R363.

Maddock, Thomas, Jr., 1969, The behavior of straight open channels with movable beds: U.S: Geol. Survey Prof. Paper 622-A, $70 \mathrm{p}$.

Mancy, K. H., and Jaffe, T., 1966, Analysis of dissolved oxygen in natural and waste waters: U.S. Dept. Health, Education, and Welfare, Public Health Service Pub. 999-WP-37, 94 p.

Mancy, K. H., and Okun, D. A., 1965, The effects of surface active agents on aeration: Water Pollution Control Federation Jour., v. 37, no. 2, p. 212-227.

Marchello, J. M., and Toor, H. L., 1963, A mixing model for transfer near a boundary: Indus. and Eng. Chemistry Fundamentals, v. 2, p. 8-12.

Merritt, C. A., McDonald, D. B., and Paulson, W. L., 1968, The effect of photosynthesis on the oxygen balance in a midwestern stream: Industrial Waste Conf., 23rd, Proc., Purdue Univ., Indiana, p. 1089-1102.

Metzger, Ivan, 1968, Surface effects in gas absorption: Environmental Sci. and Technology, v. 2, no. 10, p. 784-786.

Metzger, I., and Dobbins, W. E., 1967, Role of fluid properties in gas transfer: Environmental Sci. and Technology, v. 1 , no. 1 , p. 57-67.

Miyamoto, S., 1932a, A theory of the rate of solution of gas into liquid: Chem. Soc. Japan Bull., v. 7, p. 8-17.

$1932 \mathrm{~b}$, The rate of solution of gas into liquid containing a reacting substance, and a kinetic derivation of the distribution law, part II of A theory of the rate of solution of gas into liquid: Chem. Soc. Japan Bull., v. 7, p. 388-398.

Montgomery, H. A. C., 1967, The determination of biochemical oxygen demand by respirometric methods: Water Research, v. 1, no. 10, p. 631-662.

1969, Discussion of atmospheric oxygenation in a simulated stream: Am. Soc. Civil Engineers Jour., v. 95, no. SA-2, p. 356-358.

Montgomery, H. A. C., Thom, N. S., and Cockburn, A., 1964, Determination of dissolved oxygen by the Winkler method and the solubility of oxygen in pure water and sea water: Jour. Appl. Chemistry, v. 14, July, p. 280-296.

Moore, E. W., Thomas, H. A., Jr., and Snow, W. B., 1950, Simplified method for analysis of BOD data: Sewage and Indus. Wastes, v. 22, no. 12, p. 1343-1353.

Moore, W. A., 1938, The solubility of atmospheric oxygen in sewage: Sewage Works Jour., v. 10, no. 2, p. 241-246.

Negulescu, M., and Rojanski, V., 1969, Recent research to determine reaeration coefficient: Water Research, v. 3, no. 3, p. 189-202.

O'Connell, R. L., and Thomas, N. A., 1965, Effect of benthic algae on stream dissolved oxygen: Am. Soc. Civil Engineers Jour., v. 91, no. SA-3, p. 1-16.

O'Connor, D. J., 1967, The temporal and spatial distribution of dissolved oxygen in streams: Water Resources Research, v. 3, no. 1, p. 65-79. 
O'Connor, D. J., and Dobbins, W. E., 1958, Mechanism of reaeration in natural streams: Am. Soc. Civil Engineers Trans., v. 123, p. 641-684.

O'Connor, D. J., St. John, J. P., and DiToro, D. M., 1968, Water quality analysis of the Delaware River estuary: Am. Soc. Civil Engineers Jour., v. 94, no. SA-6, p. 12251252.

Odum, H. T., 1956, Primary production in flowing waters: Limnology and Oceanography, v. 1, p. 102-117.

Ogden, C. G., Gibbs, J. W., and Gameson, A. L. H., 1959, Some factors affecting the aeration of flowing saline water: The Water and Waste Treatment Jour., Sept.Oct., p. 392-396.

Owens, M., 1965, Some factors involved in the use of dissolved-oxygen distributions in streams to determine productivity: Istituto Italiano Idrobiologia Mem., 18 supp., p. 209-224.

Owens, M., and Edwards, R. W., 1961, Further crop studies and estimates of net productivity of macrophytes in a chalk stream, part II of The effects of plants on river conditions: Jour. Ecology, v. 49, Feb., p. 119-126.

1963, Some oxygen studies in the River Lark: Soc. Water Treatment and Exam. Proc., v. 12, p. 126-145. 1964, A chemical survey of some English rivers: Soc. Water Treatment and Exam. Proc., v. 13, p. 134-144. 1966, Some chemical aspects of water quality in relation to minimum acceptable flows: Assoc. of River Authorities Ann. Conf., Great Yarmouth, p. 3-22.

Owens, M., Edwards, R. W., and Gibbs, J. W., 1964, Some reaeration studies in streams: Internat. Jour. Air and Water Pollution, v. 8, p. 469-486.

Owens, M., and Knowles, G., 1968, The prediction of the distribution of dissolved oxygen in rivers: Water Research, v. 2, no. 1, p. 20-21.

Pescod, M. B., 1969, Photosynthetic oxygen production in a polluted tropical estuary: Water Pollution Control Federation Jour., v. 41, no. 8, p. R309-R321.

Poon, C. P. C., and Campbell, H., 1967, Diffused aeration in polluted water: Water and Sewage Works, v. 114, no. 10, p. 461-463.

Rainwater, F. H., and Thatcher, L. L., 1960, Method for collection and analysis of water samples: U.S. Geol. Survey Water-Supply Paper 1454, $301 \mathrm{p}$.

Rand, M. C., 1959, Laboratory studies of sewage effects on atmospheric reaeration: Water Pollution Control Federation Jour., v. 31, no. 10, p. 1197-1212.

Reed, L. J., and Theriault, E. J., 1931, Least squares treatment of the unimolecular expression $Y=L\left(1-e^{-K t}\right)$, part II of The statistical treatment of reaction-velocity data: Jour. Phys. Chemistry, v. 35, pt. 2, p. 950-971.

ReVelle, C. S., Loucks, D. P., and Lynn, W. R., 1967, A management model for water quality control: Water Pollution Control Federation Jour., v. 39, no. 7, p. 11641183.

1968, Linear programming applied to water quality management: Water Resources Research, v. 4, no. 1, p. 1-9.

Rolley, H. L. J., and Owens, M., 1967, Oxygen consumption rates and some chemical properties of river muds: Water Research, v. 1, nos. $11 / 12$, p. 759-766.

Sayre, W. W., 1965, Discussion of canal discharge measurements with radioisotopes: Am. Soc. Civil Engineers Jour., v. 91 , no. HY-6, p. 185-192.
Sayre, W. W., and Chang, F. M., 1968, A laboratory investigation of open-channel dispersion processes for dissolved, suspended, and floating dispersants: U.S. Geol. Survey Prof. Paper 433-E, 71 p.

Schroepfer, G. J., Robins, M. L., and Susag, R. H., 1960, A reappraisal of deoxygenation rates of raw sewage, effluents, and receiving waters: Water Pollution Control Federation Jour., v. 32, no. 11, p. 1212-1231.

Sheehy, J. P., 1960, Rapid methods for solving first-order equations: Water Pollution Control Federation Jour., v. 32 , no. 6 , p. 646-652.

Sherwood, T. K., and Pigford, R. L., 1952, Absorption and extraction: New York, McGraw-Hill Book Co., 478 p.

Snedecor, G. W., and Cochran, W. G., 1967, Statistical methods: Iowa State Univ. Press, 593 p.

Streeter, H. W., and Phelps, E. B., 1925, A study of the pollution and natural purification of the Ohio River: Washington, U.S. Public Health Service, Public Health Bull. 146, $75 \mathrm{p}$.

Streeter, H. W., Wright, C. T., and Kehr, R. W., 1936, An experimental study of atmospheric reaeration under stream-flow conditions, part III of Measures of natural oxidation in polluted streams: Sewage Works Jour., v. 8, no. 2 , p. 282-316.

Symons, James M., 1966, Discussion of effect of benthic algae on stream dissolved oxygen: Am. Soc. Civil Engineers Jour., v. 92, no. SA-1, p. 301-306.

Thackston, E. L., 1966, Longitudinal mixing and reaeration in natural streams: Nashville, Tenn., Vanderbilt Univ. Dept. Sanitary and Water Research Eng. Ph. D. dissert.

Thackston, E. L., and Krenkel, P. A., 1969a, Reaeration prediction in natural streams: Am. Soc. Civil Engineers Jour., v. 95, no. SA-1, p. 65-94.

$1969 \mathrm{~b}$, Discussion of atmospheric oxygenation in a simulated stream: Am. Soc. Civil Engineers Jour., v. 95, no. SA-2, p. 354-356.

Thayer, R. P., and Krutchkoff, R. G., 1967, Stochastic model for BOD and DO in streams: Am. Soc. Civil Engineers Jour., v. 93, no. SA-3, p. 59-72.

Theriault, E. J., 1926, The rate of deoxygenation of polluted waters, in Stream pollution - a symposium: Am. Soc. Civil Engineers Trans., v. 89, p. 1341-1350.

1927, The oxygen demand of polluted waters: Washington, U.S. Public Health Service, Public Health Bull. 173.

Thomann, R. V., 1963, Mathematical model for dissolved oxygen: Am. Soc. Civil Engineers Jour., v. 89, no. SA-5, p. 1-30.

1965, Recent results from a mathematical model of water pollution control in the Delaware estuary: Water Resources Research, v. 1, no. 3, p. 349-359.

Thomas, H. A., Jr., 1937, The "slope" method of evaluating the constants of the first-stage biochemical oxygen-demand curve: Sewage Works Jour., v. 9, no. 3, p. 425-430.

Toor, H. L., and Marchello, J. M., 1958, Film penetration model for mass and heat transfer: Am. Inst. Chem. Engineers Jour., v. 4, no. 1, p. 97-102.

Truesdale, G. A., Downing, A. L., and Lowden, G. F., 1955, The solubility of oxygen in pure water and sea water: Jour. Appl. Chemistry, v. 5, p. 53-62.

Tsivoglou, E. C., 1967, Tracer measurement of stream reaeration: Federal Water Pollution Control Adm. Rept., June, 86 p. 
Tsivoglou, E. C., Cohen, J. B., Shearer, S. D., and Godsil, P. J., 1968, Field studies, part II of Tracer measurement of stream reaeration: Water Pollution Control Federation Jour., v. 40, no. 2, pt. 1, p. 285-305.

Tsivoglou, E. C., O’Connell, R. L., Walter, C. M., Godsil, P. J., and Logsdon, G. S., 1965, Laboratory studies, part I of Tracer measurements of atmospheric reaeration: Water Pollution Control Federation Jour., v. 37, no. 10, p. 13431362.

Velz, C. J., 1958, Significance of organic sludge deposits: The Robert A. Taft Sanitary Eng. Center Technology Rept. W58-2, p. 47-57.

Velz, C. J., and Gannon, J. J., 1963, Biological extraction and accumulation in stream self-purification: Internat. Jour. Air and Water Pollution, v. 7, p. 587-595.
Wezernak, C. T., and Gannon, J. J., 1968, Evaluation of nitrification in streams: Am. Soc. Civil Engineers Jour., v. 94 , no. SA-5, no. 6159 , p. 883-895.

Whipple, W., Jr., Hunter, J. V., Davidson, B., Dittman, F., and $\mathrm{Yu}, \mathrm{S} ., 1969$, Instream aeration of polluted rivers: Water Resources Research Inst., Rutgers Univ., New Brunswick, N. J., 196 p.

Williams, W. E., 1951, Relation of BOD to stream deoxygenation: Sewage and Indus. Wastes, v. 23, no. 9, p. 1161-1175.

Wolman, Nathaniel, 1960, U.S. Senate Committee Pre-print 32, 86th Congress, 2d sess., Aug.

Yao, K. M., 1969, Discussion of reaeration prediction in natural streams: Am. Soc. Civil Engineers Jour., v. 95, no. SA-6, p. 1189-1190.

Zanoni, A. E., 1967, Waste water deoxygenation at different temperatures: Water Research, v. 1 , no. $8 / 9$, p. 543-566. 

APPENDIXES 



\section{APPENDIX A: EFFECT OF A CORROSION REACTION ON REAERATION-COEFFICIENT MEASUREMENTS IN A LABORATORY FLUME}

[Symbols used in appendix A are given in a list at the end of the appendix]

A recirculating-flume system generally consists of a headbox, a section of open channel, a tailbox, and a closed return-flow conduit. The system may be conceptualized as a flow loop consisting of an open channel and a pipe in series. If first-order oxidation reactions proceed in the channel and in the return pipe simultaneously with reaeration in the channel, the characteristic differential equations are:

$$
\text { (channel) } \quad \frac{\partial D_{1}}{\partial t}+U_{1} \frac{\partial D_{1}}{\partial x_{1}}=-K_{2} D_{1}+b_{1} C_{1}
$$

and

$$
\text { (pipe) } \quad \frac{\partial D_{2}}{\partial t}+U_{2} \frac{\partial D_{2}}{\partial x_{2}}=b_{2} C_{2},
$$

where $b$ is the first-order rate constant for the oxidation reaction, $D$ is the DO deficit, $C$ is the DO concentration, $K_{2}$ is the reaeration coefficient, $U$ is the mean water velocity, $t$ is time, $x$ is longitudinal position, and the 1 and 2 subscripts refer to the channel and pipe, respectively. These equations assume that the only source of oxygen is reaeration, the only sink is the oxidation reaction, and longitudinal dispersion effects are negligible. The oxidation reaction is assumed to follow the basic law of chemical kinetics for a first-order heterogeneous reaction; that is, the rate of reaction is proportional to the concentration of the reactant, the dissolved oxygen.

Note that $D_{1}=D_{1}\left(x_{1}, t\right)$ and $D_{2}=D_{2}\left(x_{2}, t\right)$ and also that $x_{1}=0, x_{2}=L_{2}$ designates the beginning of the channel, and $x_{1}=L_{1}, x_{2}=0$ the end of the channel. The initial and final conditions in the system are:

$$
\begin{gathered}
D_{1}(0,0)=D_{2}\left(L_{2}, 0\right)=D_{0} \\
D_{1}(0, \infty)=D_{2}\left(L_{2}, \infty\right)=D_{\varkappa},
\end{gathered}
$$

where $L_{2}$ is the length of the pipe. The solutions to equations A-1 and A-2 (derived by the authors) under these conditions are:

$$
\begin{array}{r}
D_{1}\left(x_{1}, t\right)=\frac{b_{1} C_{s}}{K_{2}+b_{1}}\left[1-e^{-\frac{\left(K_{2}+b_{1}\right) x_{1}}{U_{1}}}\right] \\
+\left(D_{0}-D_{\infty}\right) e^{-\alpha t} e^{-\left(K_{2}+b_{1}-\alpha\right)} \frac{x_{1}}{U_{1}} \\
+D_{x} e^{-\frac{\left(K_{2}+b_{1}\right) x_{1}}{U_{1}}}
\end{array}
$$

and

$$
\begin{aligned}
& D_{2}\left(x_{2}, t\right)=C_{s}\left[1-e^{-\frac{b_{2}\left(x_{2}-L_{2}\right)}{V_{2}}}\right] \\
& +\left(D_{0}-D_{x}\right) e^{-\alpha t} e^{-\frac{\left(b_{2}-\alpha\right)\left(x_{2}-L_{2}\right)}{U_{2}}}+D_{x} e^{-\frac{b_{2}}{U_{2}}\left(x_{2}-L_{2}\right)},
\end{aligned}
$$

where from

$$
D_{1}\left(L_{1}, \infty\right)=D_{2}(0, \infty)
$$

and

$$
\begin{gathered}
D_{1}\left(L_{1}, 0\right)=D_{2}(0,0): \\
D_{\infty}=\frac{C_{s}\left[\left(1-e^{+\frac{b_{2} L_{2}}{U_{2}}}\right)-\frac{b_{1}}{K_{2}+b_{1}}\left(1-e^{-\left(K_{2}+b_{1}\right) \frac{L_{1}}{U_{1}}}\right)\right]}{e^{-\left(K_{2}+b_{1} \frac{L_{1}}{U_{1}}\right.}-e^{b_{2} \frac{L_{2}}{U_{2}}}}
\end{gathered}
$$

and

$$
\alpha=\frac{Q}{V}\left[\frac{b_{2} L_{2}}{U_{2}}+\left(K_{2}+b_{1}\right) \frac{L_{1}}{U_{1}}\right]
$$

in which $V$ is the volume of the water in the system, $Q$ is the discharge of the system, $C_{s}$ is the saturation concentration, and $L_{1}$ is the length of the channel. Because of the presence of the headbox, tailbox, and pump, the dimensions $L_{1}$ and $L_{2}$ in a real flume are equivalent dimensions.

Equation A-9 gives the deficit at the station $x_{1}=0, x_{2}=L_{2}$ as $t \rightarrow \infty$. This non-zero value of the deficit is the value at which the oxygen consumed by the reactions is just replaced by that supplied by surface reaeration. Equation A-5 shows that if there is an oxygen-consuming reaction in the channel or return pipe of a flume, then because of the first term on the right of that equation and because of the non-zero $D_{\infty}$ value, the logarithm $D$ versus $t$ relation at a station $x_{1}$ will not be a straight line as shown in figure 2 . Thus the system does not follow the first-order model usually assumed valid for flume systems. Also, if $b_{1}$ and (or) $b_{2}$ are not equal to zero, the curves of $\log$ arithm $D$ versus $t$ at two different stations in the channel will not be parallel, as they would be were $b_{1}$ and $b_{2}=0$. Furthermore, it is expected, even discounting the curvature of the lines and their deviation from parallel, that the quantity $M=\frac{\Delta \ln D}{X / U}$ as determined by the method of figure 2 will be some weighted 
average of $K_{2}$ and $b_{1}$ and will not equal the reaeration coefficient, $K_{2}$. Examples presented later show that if $b_{1}=0$, the error due to the curvature and deviation from the parallel is negligible. If $b_{1} \neq 0$, equations A-5, A-9, and A-10 can be used to obtain an estimate of the true values of $K_{2}$ and $b_{1}$ from values of $M$ measured by the procedure of figure 2 .

If

$$
\begin{aligned}
D_{11}^{\prime}=D_{1}^{\prime}\left(x_{11} ; t\right)= & \frac{b_{1}}{K_{2}+b_{1}}\left[1-e^{-\left(K_{2}+b_{1}\right) \frac{x_{11}}{U_{1}}}\right]+D_{x}^{\prime} e^{-\left(K_{2}+b_{1}\right) \frac{x_{11}}{U_{1}}} \\
& +\left(D_{0}^{\prime}-D_{\infty}^{\prime}\right) e^{-\alpha t} e^{-\left(K_{2}+b_{1}-\alpha\right) \frac{x_{11}}{U_{1}}}
\end{aligned}
$$

and

$$
\begin{aligned}
D_{12}^{\prime}=D_{1}^{\prime}\left(x_{12}, t\right. & +\Delta t)=\frac{b_{1}}{K_{2}+b_{1}}\left[1-e^{-\left(K_{2}+b_{1}\right) \frac{x_{12}}{U_{1}}}\right] \\
& +D_{x}^{\prime} e^{-\left(K_{2}+b_{1}\right) \frac{x_{12}}{U_{1}}} \\
& +\left(D_{0}^{\prime}-D_{x}^{\prime}\right) e^{-\alpha(t+\Delta t)} e^{-\left(K_{2}+b_{1}-\alpha\right) \frac{x_{12}}{U_{1}}},
\end{aligned}
$$

where in the double subscripts the first subscript, 1 , indicates the channel and the second, 1 or 2 , indicates one of two longitudinal locations in the channel, $\Delta t=\frac{x_{12}-x_{11}}{U_{1}}=\frac{\Delta x}{U_{1}}$, and the deficits have been nondimensionalized by dividing by $C_{s}$, which is indicated by the primed notation, then from equations A-11 and A-12,

$$
D_{12}^{\prime}-\frac{b_{1}}{K_{2}+b_{1}}=\left(D_{11}^{\prime}-\frac{b_{1}}{K_{2}+b_{1}}\right) e^{-\frac{\Delta x}{v_{1}}\left(K_{2}+b_{1}\right)}
$$

Equation A-13 may be written

$$
D_{\mathbf{i} 2}=\delta\left(1-e^{-\eta}\right)+D_{\mathbf{i}} e^{-\eta},
$$

where $\eta=\frac{\Delta x}{U_{1}}\left(K_{2}+b_{1}\right)$ and $\delta=\frac{b_{1}}{K_{2}+b_{1}}$. Because $\eta$ is small, $e^{-\eta}$ in the first term on the right of equation A-14 is adequately represented by the first two terms in its Taylor series expansion, and equation $\mathrm{A}-14$ becomes

$$
D_{12}^{\prime}=\delta \eta+D_{11}^{\prime} e^{-\eta}
$$

Taking the natural logarithm of equation $A-15$, expressing the right-hand side in its power series form, and neglecting all powers of $\delta \eta$ greater than 1 , it may be shown that

$$
\begin{gathered}
\ln D_{12}^{\prime} \cong \ln \left(D_{11}^{\prime} e^{-\eta}\right)+\frac{\delta \eta}{D_{11}^{\prime} e^{-\eta}} . \\
\text { Because } e^{-\eta} \cong 1, \\
\Delta \ln D^{\prime}=\ln D_{11}^{\prime}-\ln D_{12}^{\prime}=\left(K_{2}+b_{1}\right) \frac{\Delta x}{U_{1}}\left[1-\frac{b_{1}}{D_{11}^{\prime}\left(K_{2}+b_{1}\right)}\right]
\end{gathered}
$$

and because $M=\Delta \ln D^{\prime} / \frac{\Delta x}{U_{1}}$,

$$
K_{2} \cong M+\frac{b_{1}}{D_{11}^{\prime}}\left(1-D_{11}^{\prime}\right) \text {. }
$$

Equation A-18 states that, assuming one has available DO-deficit measurements of two samples, one from the upstream station, and one from the downstream station at a lag time $\tau=\Delta x / U$ later, then the $K_{2}$ value as determined from $M=\Delta \ln D / \tau$ differs from the true value only if $b_{1} \neq 0$. The amount of the deviation of $M$ from $K_{2}$ increases as the deficit at the upstream station decreases. If the method of figure 2 is used, that is, if $M$ is determined not from two instantaneous point samples but from experimental results which were forced to fit two parallel straight lines, the resulting curve-fitting errors will be added to those resulting from the non-zero $b_{1}$ value.

To determine $K_{2}$ and $b_{1}$ from experimental data, a plot of $\ln D_{1}^{\prime}$ versus $t$ at two different stations, as in figure 2, should be made without forcing the data to fit two parallel straight lines. For two sufficiently different values of $D_{11}^{\prime}$, one determines $M$, as in figure 2, and computes $b_{1}$ and $K_{2}$ from two applications of equation $\mathrm{A}-18$. If the data are forced to fit two parallel straight lines, $M$ will be the same for all $D_{11}^{\prime}$, and $b_{1}$ and $K_{2}$ will be impossible to determine. Other methods for determining $K_{2}, b_{1}$, and $b_{2}$ may be derived from equations A-9, $\mathrm{A}-10$, and A-18. These methods require only a single determination of $M$, but they involve conducting the experiment until $t \rightarrow \infty$ to determine experimentally $D_{\infty}$. These methods, in addition, require measurements of $L_{1}$ and $L_{2}$, indeterminate quantities in real flume systems. Thus the procedure outlined at the start of this paragraph appears to be the best one for determining $K_{2}$ in a flume system in which a first-order oxygenconsuming reaction proceeds in the channel in conjunction with reaeration.

Equation A-18 may be rewritten as

$$
\frac{K_{2}-M}{K_{2}} \cong \frac{b_{1}}{K_{2}} \frac{\left(1-D_{11}^{\prime}\right)}{D_{11}^{\prime}},
$$

from which it is seen that if $b_{1}$ and $D_{11}^{\prime}$ are constants, the relative error in the measured $K_{2}$ increases as $K_{2}$ decreases. Thus if $b_{1}$ is a constant in a set of flume experiments, the resulting errors will be more severe at the lower end of the experimental $K_{2}$ range, with the possibility of severely biasing any resulting least-squares prediction equations. This could be a reason for the widely different values of the multiplicative constants of the regression equations obtained by Thackston and Krenkel (1969a) from the analysis of their data (Thackston, 1966; Krenkel, 1960).

The discussion above suggests a procedure for conducting a series of flume experiments for measuring $K_{2}$ which would determine if oxygenconsuming reactions are influencing the $K_{2}$ mea- 
surements. The procedure involves running a special experiment prior to the start of a test series. In this experiment, test conditions are selected which are expected to yield a $K_{2}$ value that would rank among the lowest expected in the test series. The experiment is conducted under conditions typical for the entire set, but over a larger DO-deficit range than normal-that is, for a long time. If there is no oxygen-consuming reaction in process in the system, the curves of logarithm $D$ versus $t$ observed at the two measurement stations will be straight and parallel, and $K_{2}$ may be computed by the method of figure 2 . If a reaction is in progress in the system, this will be indicated, as $t$ becomes large, by curvature and nonparallelism of the curves of logarithm $D$ versus $t$ at the two measuring stations. If the reaction is in the return conduit, $b_{1}$ as computed by the method suggested above will be zero, and as will be shown in the examples to follow, the analysis method of figure 2 will be accurate enough. If $b_{1} \neq 0, K_{2}$ must be calculated by the method given above. The trial experiment described above will point the way for conducting the other experiments in the series. If the reaction in the channel is insignificant in the test case, it will most likely also be insignificant in the others. If it were significant, its effects should be considered in the remainder of the experiments.

\section{EXAMPLES}

The usefulness of the $K_{2}$ determination procedure described above is best illustrated by example. The examples which follow utilize, with the exception of the reaction-rate constants $b_{1}$ and $b_{2}$, values of flume and flow parameters which are typical for small laboratory flumes. The values of $b_{1}$ and $b_{2}$ must necessarily be highly speculative, because available flume data are not sufficient to allow determination of typical values for these parameters. For a mixing tank, however, Tsivoglou, O'Connell, Walter, Godsil, and Logsdon (1965) reported data which indicate that the reactionrate constants in a largely metal system can be on the order of $2 \times 10^{-5}$ per second. The reaction is most likely due to corrosion of the walls of the system and would be expected to vary widely from one system to another; consequently, slightly larger values for $b_{1}$ and $b_{2}$ have been chosen for use in the examples described. The relative error in a measured $K_{2}$ due to a particular value of $b_{1}$ is dependent on the ratio $b_{1} / K_{2}$, so that for the values of $b_{1}$ used in the examples, the relative errors will be more or less severe for respectively larger or smaller $K_{2}$ values.
In the examples, DO deficits at two sampling stations were calculated using equation A-5 and the parameters listed in table 17 , where $\alpha$ and $D_{\infty}$ were calculated from equations A-9 and A-10.

TABLE 17. - Parameters used in calculating the synthetic flume data and the resulting calculated reaeration coefficient

\begin{tabular}{|c|c|c|c|c|c|c|c|}
\hline $\begin{array}{c}\text { Example } \\
\text { No. }\end{array}$ & $\begin{array}{c}K_{2} \\
\frac{1}{\sec } \\
\times 10^{4}\end{array}$ & $\begin{array}{c}b_{1} \\
\frac{1}{\sec } \\
\times 10^{5}\end{array}$ & 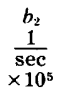 & $\begin{array}{c}\alpha \\
\frac{1}{\mathrm{sec}} \\
\times 10^{4}\end{array}$ & $\mathbf{D}_{\mathrm{u}}$ & $D_{x}$ & $\begin{array}{c}M \\
\frac{1}{\mathrm{sec}} \\
\times 10^{4}\end{array}$ \\
\hline 1 & 8.00 & 0 & 8.00 & 3.50 & 0.800 & 0.144 & 8.00 \\
\hline 2 & 8.00 & 0 & 8.00 & 3.50 & .400 & $\begin{array}{r}.144 \\
.144\end{array}$ & 8.00 \\
\hline 3 & 8.00 & 4.00 & 8.00 & 3.65 & .800 & .179 & 7.81 \\
\hline 4 & 8.00 & 4.00 & 8.00 & 3.65 & .400 & .179 & 7.27 \\
\hline 5 & 4.00 & 4.00 & 4.00 & 1.90 & .400 & .211 & 3.34 \\
\hline
\end{tabular}

From the synthetic flume data, $M$ was calculated for each of the examples by the method of figure 2. Least-squares straight lines were fit to 20 minute increments of the calculated values of the logarithm deficit and $t$ for each of the two sampling stations. The 20-minute sampling period was chosen because this was the period used by both Thackston (1966) and Krenkel (1960). The slopes of the two lines were averaged, and new intercepts were computed for parallel least-squares lines through the two sets of points. The quantity $M$ was then computed from the new intercepts and the average slope. These calculated $M$ values are listed in table 17. In this procedure, $M$ is not a function of time as it is if the lines are not parallel.

Figure 18 shows plots of logarithm $D$ versus $t$ for the upstream sampling stations in examples 3 , 4, and 5; the lines are the least-squares fits to the synthetic data points. The curvature due to the non-zero $D_{x}$ value is virtually unnoticeable over the 20-minute time period. In fact, if experimental data with its scatter were used, the curvature would probably be impossible to detect. Thus, over a 20-minute experimental time, it is virtually impossible to detect a non-zero $D_{x}$, and it is impossible to conclude whether or not an oxygen consuming reaction is proceeding in the system. As can be seen from table 17 , for examples 3,4 , and 5 , the deviation of $M$, the calculated $K_{2}$, from the true value is significant. Thus it is possible for oxygen-consuming reactions to cause significant errors in calculated $K_{2}$ values without their presence being noticed in the experimental results.

The values of $M$ for examples 1 and 2 are not significantly different from $8.0 \times 10^{-4}$ seconds $^{-1}$. This shows that, at least for short time periods, if 


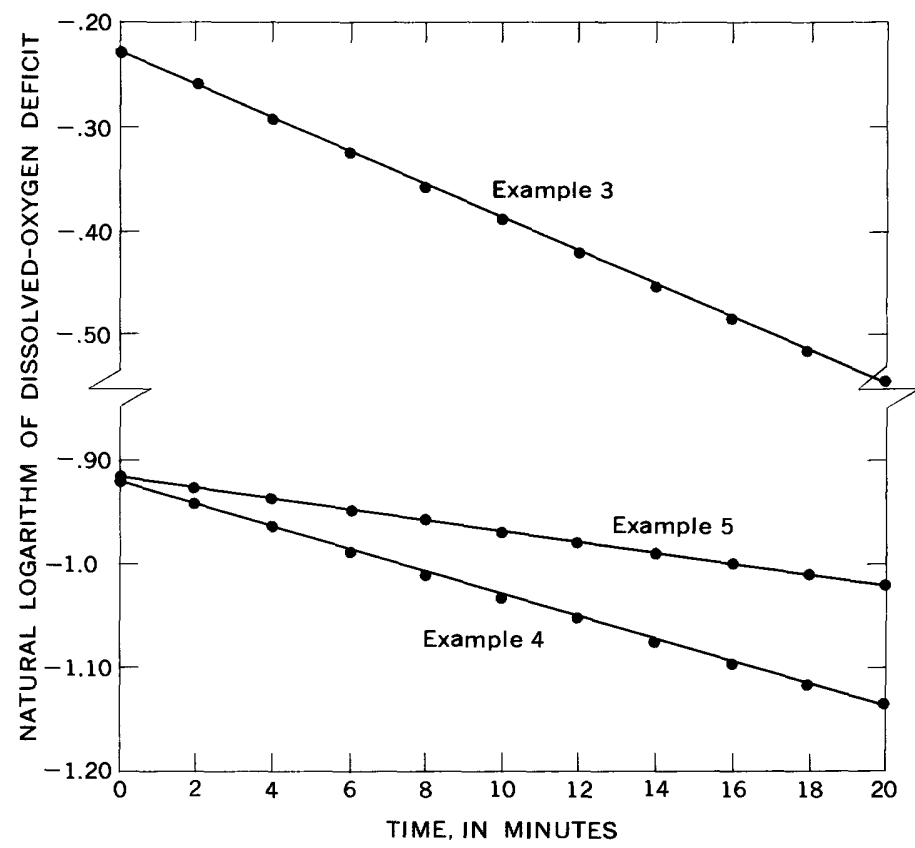

Figure 18. - Synthetic flume data from the upstream sampling station.

$b_{1}$ is zero, the deviation of $M$ from the true $K_{2}$, due to the curvature induced by a non-zero $D_{\infty}$, is negligible. Thus, when $C_{s}$ is known, the $K_{2}$ calculation procedure illustrated in figure 2 will contain significant errors only if $b_{1}$ is not zero.

The upstream deficit values at $t=0$ minutes for examples 3,4 , and 5 , respectively, are $0.676,0.356$, and 0.379 . The corresponding values of $M$ calculated from equation A-18 are $7.82,7.28$, and $3.34 \times 10^{-4}$ seconds $^{-1}$. These are very nearly equal to the $M$ values calculated by the procedure of figure 2 listed in column 8 of table 17 . Even better correspondence between the measured $M$ and the $M$ calculated from equation A-18 is obtained if instantaneous values of the upstream and downstream deficits are used to calculate $M$. For instance, in example 5 at $t=16$ minutes the upstream deficit is 0.368 , and the $M$ value calculated from the deficits is $3.31 \times 10^{-4}$ seconds $^{-1}$, whereas the $M$ calculated from equation A-18 is $3.31 \times 10^{-4}$ seconds ${ }^{-1}$. The close correspondence of the $M$ values obtained in the two ways indicates that despite the approximations made in its derivation, equation A-18 provides an accurate means for obtaining $K_{2}$ and $b_{1}$ from two measured values of $M$.

The procedure for determining $K_{2}$ and $b_{1}$ from experimental data is illustrated in figure 19. Synthetic data parameters shown in figure 19 are the same as those for example 4 in table 17. Deficits at the upstream and downstream sampling stations are plotted for two periods: from 0 to 10 minutes and from 50 to 60 minutes. The procedure consists of plotting the natural logarithm of deficit versus time, determining $M$ graphically (as in fig. 2) for each of the 10 minute periods, and solving for $b_{1}$ and $K_{2}$ using equation A-18 and the resulting two sets of $D_{11}^{\prime}$ and $M$ values. For the example given in figure $18, D_{11}^{\prime}$ and $M$ for the initial 10 minute period are, respectively, 0.376 and $7.20 \times 10^{-4}$ seconds ${ }^{-1}$, whereas for the period from 50 to 60 minutes, they are 0.245 and $6.56 \times 10^{-4}$ seconds $^{-1}$. Using equation A-18, the calculated $b_{1}$ and $K_{2}$ values are, respectively, $4.51 \times 10^{-4}$ seconds $^{-1}$ and $7.95 \times 10^{-4}$ second $\mathrm{s}^{-1}$. The agreement of these values with the $b_{1}$ and $K_{2}$ values used in generating the synthetic data is very good, considering that the two $M$ values were determined graphically.

If in a flume study there is an error in the value of $C_{s}$ being used, there will be a corresponding error in all the computed deficits. In such a case, the true deficit, $D_{T}$ is related to the plotted deficit by $D=D_{T}+d$. The resulting equation for the plotted deficit, assuming no oxygen-consuming reactions in either the channel or the return conduit, is

$$
D=d+\left(D_{T_{0}}-d\right) e^{-\alpha t} e^{-\left(\kappa_{2}-\alpha\right) \frac{x}{U_{1}}}
$$

Equation A-21 is similar to equation A-5, with $b_{1}=0$. It has been shown that in the case of $b_{1}=0$, the error in the $K_{2}$ computed using the method of figure 2 is negligible. Thus in a flume study, a negligible error is introduced by using a value of $C_{s}$ which is incorrect by a small amount $\Delta d$. This is in contrast to the nature of errors in analysis of mixing-tank data where $K_{2}$ is determined from the slope of a single line (Isaacs and Gaudy, 1968; Tsivoglou and others, 1965).

\section{SYMBOLS USED IN APPENDIX A}

\footnotetext{
Symbol

Definition

$b_{1} \quad$ Rate constant for the first-order oxidation reaction in the channel of the flume, in reciprocal seconds.

$b_{2} \quad$ Rate constant for the first-order oxidation reaction in the return pipe of the flume, in reciprocal seconds.

$C_{1}$ Dissolved-oxygen concentration in the water in the channel of the flume, in milligrams per liter.

$C_{2} \quad$ Dissolved-oxygen concentration in the water in the return pipe of the flume, in milligrams per liter.

$C_{s} \quad$ Dissolved-oxygen concentration at saturation, in milligrams per liter.

$D$ Computed deficit of dissolved oxygen, in milligrams per liter.
} 
APPENDIX A

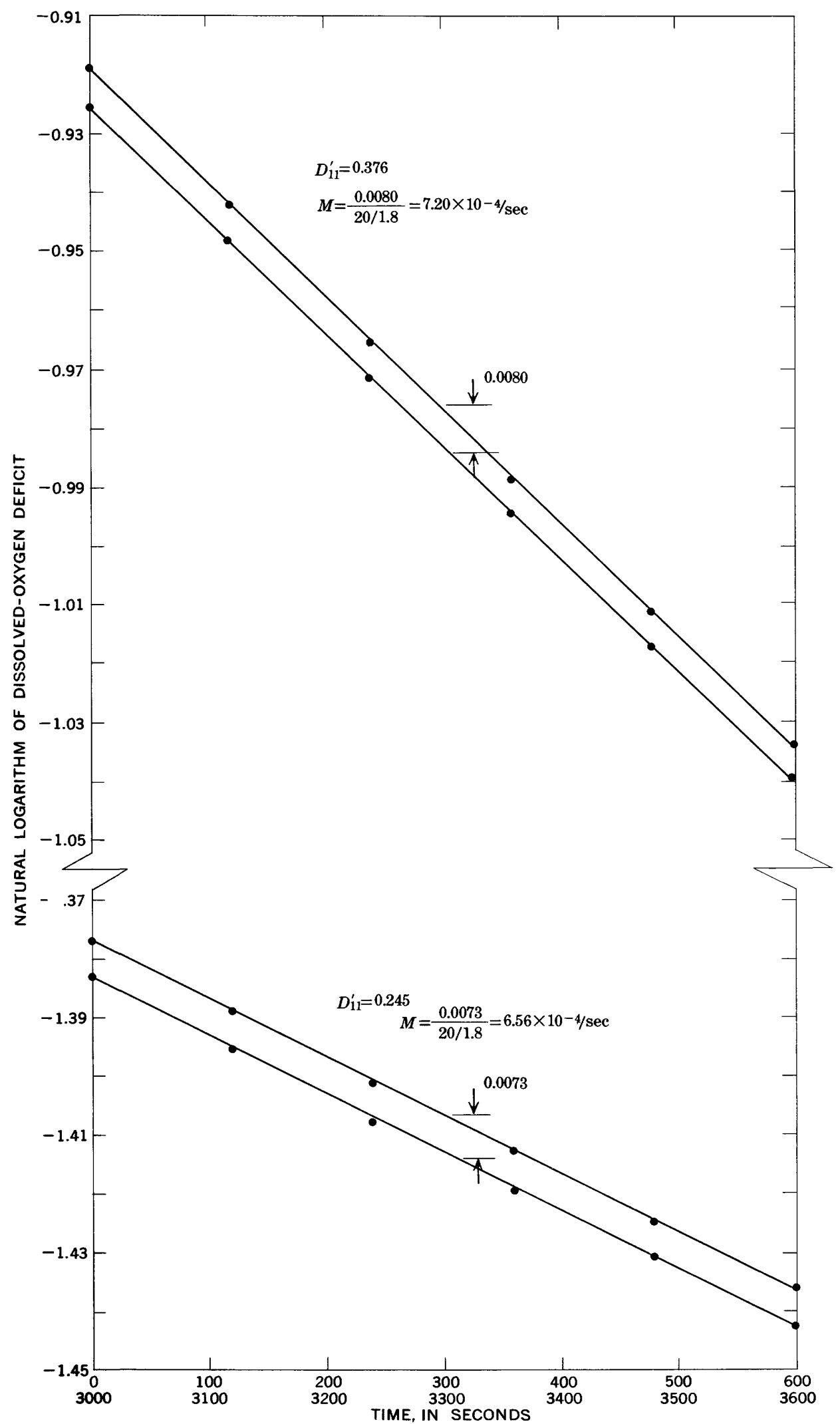

Figure 19. - Determination of $b_{1}$ and $K_{2}$ from $M$ values calculated at two different DO deficits. 
$D_{1} \quad$ Equivalent to $D_{1}\left(x_{1}, t\right)$, the dissolved-oxygen deficit at longitudinal position $x$ in the channel of the flume at time $t$, in milligrams per liter. (Subscript 1 indicates the channel of the flume.)

$D_{2} \quad$ Equivalent to $D_{2}\left(x_{2}, t\right)$, the dissolved-oxygen deficit at longitudinal position $x$ in the return pipe of the flume at time $t$, in milligrams per liter. (Subscript 2 indicates the return pipe of the flume.)

$D_{0} \quad$ Initial dissolved-oxygen deficit in the system at time $t=0$, in milligrams per liter.

$D_{\infty} \quad$ Final dissolved-oxygen deficit in the system at time $t=\infty$, in milligrams per liter.

$D_{0}^{\prime} \quad$ Nondimensional deficit of dissolved oxygen in the system at time $t=0$, equal to $D_{0} / C_{s}$.

$D_{x}^{\prime} \quad$ Nondimensional deficit of dissolved oxygen in the system at time $t=\infty$, equal to $D_{x} / C_{s}$.

$D_{T} \quad$ True deficit of dissolved oxygen, equal to the computed deficit minus an error, or $D-d$, in milligrams per liter.

$D_{T_{0}} \quad$ Initial true deficit of dissolved oxygen, equal to the initial computed deficit minus an error, or $D_{0}-d$, in milligrams per liter.

$D_{11}^{\prime} \quad$ Equivalent to $D_{1}^{\prime}\left(x_{11}, t\right)$, the nondimensional deficit of dissolved oxygen in the channel at longitudinal position $x_{1}$ at time $t$. (Deficit nondimensionalized by dividing by $C_{s}$; first subscript in the double subscript (1) indicates the channel of the flume, and the second subscript (1) indicates upstream measurement point in the channel.)

$D_{12}^{\prime} \quad$ Equivalent to $D_{1}^{\prime}\left(x_{12}, t+\Delta t\right)$, the nondimensional deficit of dissolved oxygen in the channel at longitudinal position $x_{2}$ at time $t+\Delta t$. (Deficit nondimensionalized by dividing by $C_{s}$; first subscript in the double subscript (1) indicates the channel of the flume, and the second subscript (2) indicates downstream measurement point in the channel; $\Delta t$ is the flow time or $\left(x_{12}-x_{11}\right) / U_{1}$, where $U_{1}$ is the mean water velocity in the channel.)

$d \quad$ Error in the saturation concentration of dissolved oxygen, in milligrams per liter.

$K_{2}$ Reaeration coefficient or rate constant for oxygen absorption from the atmosphere, natural logarithm base, in reciprocal days.

$L_{1} \quad$ Length of the flume channel, in feet. (Equivalent dimension because of the presence of the headbox and tailbox.)

$L_{2} \quad$ Length of the return pipe, in feet. (Equivalent dimension because of the presence of the headbox, tailbox, and pump.)

$M$

Equal to $\Delta \ln D / \frac{X}{U}$ (nomenclature of fig. 2 and the main part of the report) or $\Delta \ln D^{\prime} / \frac{\Delta x}{U_{1}}$ (nomenclature of appendix A); $X \equiv \Delta x=$ the distance, in feet, between the upstream and downstream measurement points in the flume channel; $U \equiv U_{1}=$ the mean water velocity, in feet per second, in the flume channel; $\Delta \ln D \equiv \Delta \ln D^{\prime}=$ the difference in the natural logarithms of the dissolved-oxygen deficits at the upstream and downstream measurement points with the downstream measurement lagging the upstream by the flow time, $\Delta x / U_{1}$. See figure 2 .

$Q \quad$ Water discharge, in cubic feet per second.

$t \quad$ Time, in seconds.

$U_{1} \quad$ Mean water velocity in the channel of the flume, in feet per second.

$U_{2} \quad$ Mean water velocity in the return pipe of the flume, in feet per second.

$x_{1} \quad$ Longitudinal position in the channel of the flume, in feet.

$x_{11} \quad$ Upstream measurement point in the channel of the flume, in feet.

$x_{12}$ Downstream measurement point in the channel of the flume, in feet. Equal to $\frac{Q}{V}\left[\frac{b_{2} L_{2}}{U_{2}}+\left(K_{2}+b_{1}\right) \frac{L_{1}}{U_{1}}\right]$, in reciprocal seconds.

$\delta \quad$ Equal to $b_{1} /\left(K_{2}+b_{1}\right)$.

$\eta \quad$ Equal to $\frac{\Delta x}{U_{1}}\left(K_{2}+b_{1}\right)$.

$\tau$ Lag time or flow time, equal to $\Delta x / U_{1}$, in seconds. 
APPENDIX B: COLLECTED DATA

TABLE 18. - Reaeration coefficients and hydraulic data from Churchill, Elmore, and Buckingham (1962)

\begin{tabular}{|c|c|c|c|c|}
\hline $\begin{array}{c}k_{2} \dagger \\
\left(d_{a y}-1\right)\end{array}$ & $\begin{array}{c}H \\
\text { (feet) }\end{array}$ & $\underset{(\text { fps) }}{U}$ & $\frac{S}{\left(\times 10^{\prime}\right)}$ & $\underset{\text { (feet) }}{W}$ \\
\hline $\begin{array}{r}2.272 \\
1.440 \\
.981 \\
.496 \\
.743\end{array}$ & $\begin{array}{l}3.27 \\
5.09 \\
4.42 \\
6.14 \\
5.66\end{array}$ & $\begin{array}{l}3.07 \\
3.69 \\
2.10 \\
2.68 \\
2.78\end{array}$ & $\begin{array}{r}10.8502 \\
8.6426 \\
2.7058 \\
2.9014 \\
3.6970\end{array}$ & $\begin{array}{l}229 \\
242 \\
344 \\
358 \\
376\end{array}$ \\
\hline $\begin{array}{l}1.129 \\
.281 \\
3.361 \\
2.794 \\
1.568\end{array}$ & $\begin{array}{r}7.17 \\
11.41 \\
2.12 \\
2.93 \\
4.54\end{array}$ & $\begin{array}{l}2.64 \\
2.92 \\
2.47 \\
3.44 \\
4.65\end{array}$ & $\begin{array}{r}1.6448 \\
1.7994 \\
14.2342 \\
14.5225 \\
13.9099\end{array}$ & $\begin{array}{l}313 \\
312 \\
617 \\
635 \\
667\end{array}$ \\
\hline $\begin{array}{l}.455 \\
.389 \\
.270 \\
.550 \\
.544\end{array}$ & $\begin{array}{l}9.50 \\
6.29 \\
7.52 \\
7.07 \\
\mathbf{5 . 4 4}\end{array}$ & $\begin{array}{l}2.94 \\
2.51 \\
3.15 \\
3.30 \\
3.11\end{array}$ & $\begin{array}{l}1.6893 \\
2.9038 \\
3.0443 \\
3.7775 \\
5.9961\end{array}$ & $\begin{array}{l}374 \\
414 \\
443 \\
450 \\
330\end{array}$ \\
\hline $\begin{array}{r}.604 \\
1.251 \\
.273 \\
.225 \\
1.881\end{array}$ & $\begin{array}{r}8.06 \\
3.88 \\
9.28 \\
10.19 \\
3.29\end{array}$ & $\begin{array}{l}4.28 \\
2.73 \\
2.41 \\
3.06 \\
2.40\end{array}$ & $\begin{array}{r}5.7667 \\
6.0706 \\
1.2571 \\
1.7789 \\
15.5378\end{array}$ & $\begin{array}{r}346 \\
90 \\
537 \\
549 \\
520\end{array}$ \\
\hline $\begin{array}{l}.842 \\
.883 \\
.915 \\
.995 \\
.547\end{array}$ & $\begin{array}{l}4.74 \\
5.72 \\
6.95 \\
4.29 \\
6.01\end{array}$ & $\begin{array}{l}3.46 \\
4.02 \\
4.52 \\
1.85 \\
2.75\end{array}$ & $\begin{array}{r}13.5458 \\
11.7032 \\
10.1594 \\
3.9709 \\
3.7523\end{array}$ & $\begin{array}{l}\mathbf{5 3 5} \\
\mathbf{5 4 2} \\
\mathbf{5 5 0} \\
517 \\
531\end{array}$ \\
\hline $\begin{array}{r}.881 \\
.252 \\
5.558 \\
1.712 \\
3.222 \\
\end{array}$ & $\begin{array}{l}7.16 \\
8.49 \\
3.42 \\
3.02 \\
2.83 \\
\end{array}$ & $\begin{array}{l}3.23 \\
3.71 \\
5.00 \\
3.05 \\
3.51 \\
\end{array}$ & $\begin{array}{r}3.6794 \\
3.6430 \\
23.5142 \\
17.4603 \\
18.8692 \\
\end{array}$ & $\begin{array}{l}539 \\
548 \\
182 \\
124 \\
115\end{array}$ \\
\hline
\end{tabular}

$\dagger$ Adjusted to $\left(k_{2}\right)_{20^{\circ}}$, using equation 104.
TABLE 19. - Reaeration coefficients and hydraulic data from Owens, Edwards, and Gibbs (1964)

\begin{tabular}{|c|c|c|c|c|}
\hline $\begin{array}{c}k_{2 \dagger} \dagger \\
\left(d_{2} y^{-1}\right)\end{array}$ & $\underset{\text { (feet) }}{H}$ & $\underset{\text { (fps) }}{U}$ & $\begin{array}{c}S \\
\left(\times 10^{4}\right)\end{array}$ & $\begin{array}{c}W \\
\text { (feet) }\end{array}$ \\
\hline $\begin{array}{l}2.35 \\
2.06 \\
3.20 \\
2.37 \\
4.57\end{array}$ & $\begin{array}{l}1.21 \\
1.15 \\
1.09 \\
1.50 \\
1.08\end{array}$ & $\begin{array}{r}0.14 \\
.14 \\
.13 \\
.37 \\
.16\end{array}$ & $\begin{array}{l}3.94 \\
3.94 \\
3.94 \\
3.94 \\
4.24\end{array}$ & $\begin{array}{l}28.7 \\
25.8 \\
27.3 \\
27.7 \\
28.1\end{array}$ \\
\hline $\begin{array}{r}2.09 \\
1.18 \\
3.18 \\
6.18 \\
.90\end{array}$ & $\begin{array}{r}.98 \\
1.12 \\
1.46 \\
1.31 \\
2.44\end{array}$ & $\begin{array}{l}.15 \\
.13 \\
.38 \\
.47 \\
.23\end{array}$ & $\begin{array}{r}4.24 \\
4.24 \\
4.24 \\
11.65 \\
8.95\end{array}$ & $\begin{array}{l}28.2 \\
26.6 \\
27.8 \\
25.0 \\
17.9\end{array}$ \\
\hline $\begin{array}{r}1.66 \\
.75 \\
2.12 \\
1.41 \\
.31\end{array}$ & $\begin{array}{l}2.03 \\
1.74 \\
1.47 \\
1.82 \\
2.41\end{array}$ & $\begin{array}{l}.22 \\
.28 \\
.37 \\
.50 \\
.43\end{array}$ & $\begin{array}{l}4.08 \\
2.69 \\
8.72 \\
1.56 \\
1.56\end{array}$ & $\begin{array}{l}22.5 \\
22.5 \\
20.1 \\
39.8 \\
34.9\end{array}$ \\
\hline $\begin{array}{l}31.80 \\
24.53 \\
24.57 \\
25.59 \\
28.34\end{array}$ & $\begin{array}{l}.72 \\
.87 \\
.85 \\
.40 \\
.40\end{array}$ & $\begin{array}{r}1.37 \\
1.19 \\
1.07 \\
.44 \\
.56\end{array}$ & $\begin{array}{r}106.00 \\
84.00 \\
61.00 \\
92.00 \\
61.50\end{array}$ & $\begin{array}{l}21.9 \\
20.9 \\
23.7 \\
15.3 \\
12.1\end{array}$ \\
\hline $\begin{array}{l}22.80 \\
49.17 \\
30.77 \\
18.46 \\
21.05\end{array}$ & $\begin{array}{r}.39 \\
.60 \\
.69 \\
1.00 \\
.82\end{array}$ & $\begin{array}{r}.63 \\
1.83 \\
1.81 \\
1.54 \\
1.07\end{array}$ & $\begin{array}{r}60.50 \\
92.00 \\
61.50 \\
60.50 \\
101.60\end{array}$ & $\begin{array}{l}11.0 \\
16.1 \\
14.2 \\
11.5 \\
21.8\end{array}$ \\
\hline $\begin{array}{l}16.06 \\
12.04 \\
20.32 \\
18.90 \\
20.25 \\
17.09 \\
19.16\end{array}$ & $\begin{array}{l}.78 \\
.64 \\
.48 \\
.72 \\
.66 \\
.67 \\
.69\end{array}$ & $\begin{array}{r}1.27 \\
.46 \\
.60 \\
1.16 \\
1.31 \\
1.30 \\
1.25\end{array}$ & $\begin{array}{l}83.00 \\
61.20 \\
65.70 \\
61.20 \\
\mathbf{6 5 . 7 0} \\
\mathbf{6 5 . 7 0} \\
\mathbf{6 5 . 7 0}\end{array}$ & $\begin{array}{l}19.3 \\
17.3 \\
17.7 \\
20.7 \\
20.0 \\
19.9 \\
20.0\end{array}$ \\
\hline
\end{tabular}

$\dagger$ Adjusted to $\left(k_{2}\right) 20^{\circ}$, using equation 104 . 
TABLE 20. - Reaeration coefficients and hydraulic data from Gameson, Truesdale, and Downing (1955)

\begin{tabular}{cccc}
\hline $\begin{array}{c}k_{2} \dagger \\
\text { (day-1) }\end{array}$ & $\begin{array}{c}H \\
\text { (feet) }\end{array}$ & $\begin{array}{c}U \\
\text { (fps) }\end{array}$ & $\begin{array}{c}W \\
\text { (feet) }\end{array}$ \\
\hline 57.7 & 0.41 & 1.04 & 5.7 \\
48.7 & .41 & 1.12 & 5.3 \\
55.6 & .42 & .99 & 5.6 \\
46.2 & .42 & 1.02 & 5.4 \\
34.3 & .42 & .73 & 6.0 \\
35.2 & .34 & .74 & 6.0 \\
\hline
\end{tabular}

†Adjusted to $\left(k_{2}\right)_{20^{\circ}}$, using equation 104 .

TABLE 21. - Reaeration coefficients and hydraulic data from O'Connor and Dobbins (1958) (Streeter and Phelps, 1925)

\begin{tabular}{|c|c|c|c|}
\hline$\left(\begin{array}{c}k_{2} \dagger \\
\left(\mathrm{day}^{-1}\right)\end{array}\right.$ & $\begin{array}{c}H \\
\text { (feet) }\end{array}$ & $\begin{array}{c}U \\
(f \text { ps })\end{array}$ & $\underset{\left(\times 10^{4}\right)}{S}$ \\
\hline $\begin{array}{r}0.45 \\
.23 \\
.15 \\
.34 \\
.17\end{array}$ & $\begin{array}{l}9.2 \\
8.8 \\
9.3 \\
6.5 \\
7.0\end{array}$ & $\begin{array}{r}2.32 \\
1.98 \\
2.18 \\
1.41 \\
.59\end{array}$ & $\begin{array}{r}0.68 \\
.68 \\
.68 \\
.68 \\
1.97\end{array}$ \\
\hline $\begin{array}{l}.26 \\
.20 \\
.14 \\
.19 \\
.37\end{array}$ & $\begin{array}{l}5.8 \\
5.3 \\
4.8 \\
6.4 \\
4.0\end{array}$ & $\begin{array}{l}.32 \\
.22 \\
.19 \\
.43 \\
.23\end{array}$ & $\begin{array}{l}1.97 \\
1.97 \\
1.97 \\
1.23 \\
1.23\end{array}$ \\
\hline $\begin{array}{l}.22 \\
.71 \\
.15 \\
.20 \\
.26\end{array}$ & $\begin{array}{r}8.6 \\
7.6 \\
7.1 \\
5.5 \\
13.8\end{array}$ & $\begin{array}{r}.40 \\
.40 \\
.22 \\
.51 \\
2.50\end{array}$ & $\begin{array}{r}.95 \\
.95 \\
.95 \\
1.67 \\
1.97\end{array}$ \\
\hline $\begin{array}{l}.22 \\
.31 \\
.21 \\
.23 \\
.86\end{array}$ & $\begin{array}{r}15.0 \\
8.6 \\
7.2 \\
6.6 \\
14.3\end{array}$ & $\begin{array}{r}4.20 \\
1.36 \\
.86 \\
.53 \\
3.68\end{array}$ & $\begin{array}{r}1.23 \\
1.23 \\
1.23 \\
1.23 \\
.95\end{array}$ \\
\hline $\begin{array}{l}.92 \\
.45 \\
.19 \\
.24\end{array}$ & $\begin{array}{r}9.3 \\
9.0 \\
18.7 \\
8.8\end{array}$ & $\begin{array}{r}1.01 \\
.62 \\
3.72 \\
1.48\end{array}$ & $\begin{array}{r}.95 \\
.95 \\
1.67 \\
1.67\end{array}$ \\
\hline $\begin{array}{l}.17 \\
.19 \\
.16 \\
.14\end{array}$ & $\begin{array}{r}8.2 \\
7.2 \\
7.3 \\
24.2 \\
\end{array}$ & $\begin{array}{r}1.18 \\
.91 \\
.96 \\
3.90\end{array}$ & $\begin{array}{r}1.67 \\
1.67 \\
1.67 \\
.68\end{array}$ \\
\hline
\end{tabular}

TABLE 22. - Reaeration coefficients and hydraulic data from O'Connor and Dobbins (1958) (various sources)

\begin{tabular}{cccc}
\hline $\begin{array}{c}k_{2} \dagger \\
\text { (day-1) }\end{array}$ & $\begin{array}{c}H \\
\text { (feet) }\end{array}$ & $\begin{array}{c}U \\
\text { (fps) }\end{array}$ & $\begin{array}{c}S \\
\left(\times 10^{4}\right)\end{array}$ \\
\hline 5.80 & 0.9 & 0.97 & 36 \\
3.07 & 1.9 & .55 & 14 \\
1.22 & 4.0 & .73 & 4.9 \\
.92 & 3.1 & .65 & 6.9 \\
.23 & 9.2 & 1.37 & .27 \\
.28 & 9.0 & 1.57 & .27 \\
.30 & 8.9 & 1.63 & .27 \\
.048 & 12 & .32 & $\ldots$ \\
.018 & 32 & .50 & $\ldots$ \\
.026 & 37 & .88 & $\ldots$ \\
\hline
\end{tabular}

†Adjusted to $\left(k_{2}\right)_{20}$, using equation 104.

TABLE 23. - Reaeration coefficients and hydraulic data from Tsivoglou and others $(1967,1968)$

\begin{tabular}{cccc}
\hline $\begin{array}{c}k_{2} \dagger \\
\text { (day-1) }\end{array}$ & $\begin{array}{c}H \\
\text { (feet) }\end{array}$ & $\begin{array}{c}U \\
\text { (fps) }\end{array}$ & $\begin{array}{c}W \\
\text { (feet) }\end{array}$ \\
\hline 1.273 & 1.76 & 0.636 & 73.7 \\
1.142 & 1.94 & .419 & 101.5 \\
.977 & 2.09 & .359 & 110.0 \\
.646 & 3.11 & .458 & 57.9 \\
1.653 & 1.67 & .570 & 86.7 \\
.872 & 2.36 & .346 & 101.0 \\
1.251 & 1.99 & .335 & 123.8 \\
1.170 & 2.26 & .436 & 83.72 \\
1.261 & 2.83 & .286 & 101.9 \\
3.373 & 2.30 & .563 & 63.7 \\
1.163 & 1.95 & .529 & 80 \\
1.491 & 2.42 & .441 & 77.3 \\
2.344 & 2.13 & .539 & 71.9 \\
1.420 & 2.06 & .425 & 94.2 \\
.977 & 1.66 & .457 & 108.8 \\
\hline
\end{tabular}

$\dagger$ Adjusted to $\left(k_{2}\right)_{20^{\circ}}$, using equation 104.

TABLE 26. - Reaeration coefficients and hydraulic data from Negulescu and Rojanski (1969)

\begin{tabular}{cccc}
\hline $\begin{array}{c}k_{2} \dagger \\
\text { (day-1) }\end{array}$ & $\begin{array}{c}H \\
\text { (feet) }\end{array}$ & $\begin{array}{c}U \\
\text { (fps) }\end{array}$ & $\begin{array}{c}W \\
\text { (feet) }\end{array}$ \\
\hline 15.90 & 0.492 & 1.902 & 0.66 \\
11.50 & .492 & 1.640 & .66 \\
17.25 & .164 & .951 & .66 \\
18.70 & .164 & .853 & .66 \\
8.64 & .492 & 1.050 & .66 \\
11.50 & .492 & .656 & .66 \\
8.64 & .492 & .886 & .66 \\
14.45 & .361 & 1.082 & .66 \\
\hline
\end{tabular}

tAdjusted to $\left(k_{2}\right) 20^{\circ}$, using equation 104 . 
TABLE 24. - Reaeration coefficients and hydraulic data from Thackston (1966)

\begin{tabular}{|c|c|c|c|c|}
\hline $\begin{array}{c}k_{2} \ddagger \\
\left(\mathrm{day}^{-1}\right)\end{array}$ & $\begin{array}{c}H \\
\text { (feet) }\end{array}$ & $\begin{array}{c}U \\
\text { (fps) }\end{array}$ & $\begin{array}{c}S \\
\left(\times 10^{4}\right)\end{array}$ & $\begin{array}{c}W \\
\text { (feet) }\end{array}$ \\
\hline $\begin{array}{l}18.72 \\
20.75 \\
25.45 \\
34.85 \\
40.6\end{array}$ & $\begin{array}{r}0.125 \\
.109 \\
.124 \\
.092 \\
.098\end{array}$ & $\begin{array}{l}1.323 \\
1.449 \\
1.878 \\
1.707 \\
1.858\end{array}$ & $\begin{array}{r}59.6 \\
89.4 \\
116.1 \\
144.6 \\
162.3\end{array}$ & $\begin{array}{l}2.0 \\
2.0 \\
2.0 \\
2.0 \\
2.0\end{array}$ \\
\hline $\begin{array}{l}44.9 \\
52.5 \\
24.35 \\
19.57 \\
18.38\end{array}$ & $\begin{array}{l}.122 \\
.117 \\
.170 \\
.112 \\
.104\end{array}$ & $\begin{array}{r}2.320 \\
2.029 \\
2.079 \\
1.225 \\
.994\end{array}$ & $\begin{array}{r}178.5 \\
141.8 \\
84.3 \\
55.7 \\
43.0\end{array}$ & $\begin{array}{l}2.0 \\
2.0 \\
2.0 \\
2.0 \\
2.0\end{array}$ \\
\hline $\begin{array}{r}21.1 \\
15.39 \\
16.69 \\
9.98 \\
13.19\end{array}$ & $\begin{array}{l}.107 \\
.094 \\
.107 \\
.113 \\
.099\end{array}$ & $\begin{array}{l}.921 \\
.802 \\
.940 \\
.773 \\
.776\end{array}$ & $\begin{array}{l}38.0 \\
23.6 \\
42.3 \\
24.5 \\
28.2\end{array}$ & $\begin{array}{l}2.0 \\
2.0 \\
2.0 \\
2.0 \\
2.0\end{array}$ \\
\hline $\begin{array}{l}26.15 \\
39.55 \\
40.55 \\
43.8 \\
31.90\end{array}$ & $\begin{array}{l}.065 \\
.065 \\
.063 \\
.072 \\
.052\end{array}$ & $\begin{array}{r}.754 \\
1.116 \\
.961 \\
1.069 \\
.678\end{array}$ & $\begin{array}{r}57.1 \\
112.4 \\
89.4 \\
89.7 \\
67.4\end{array}$ & $\begin{array}{l}2.0 \\
2.0 \\
2.0 \\
2.0 \\
2.0\end{array}$ \\
\hline $\begin{array}{l}39.45 \\
16.74 \\
16.32 \\
36.45 \\
14.62\end{array}$ & $\begin{array}{l}.078 \\
.082 \\
.085 \\
.070 \\
.101\end{array}$ & $\begin{array}{l}.848 \\
.802 \\
.365 \\
.389 \\
.645\end{array}$ & $\begin{array}{l}52.4 \\
43.8 \\
31.5 \\
60.0 \\
65.9\end{array}$ & $\begin{array}{l}2.0 \\
2.0 \\
2.0 \\
2.0 \\
2.0\end{array}$ \\
\hline $\begin{array}{l}21.95 \\
25.87 \\
24.25 \\
24.72 \\
64.05\end{array}$ & $\begin{array}{l}.119 \\
.146 \\
.082 \\
.129 \\
.057\end{array}$ & $\begin{array}{r}.865 \\
1.100 \\
.636 \\
1.110 \\
.558\end{array}$ & $\begin{array}{r}79.4 \\
79.1 \\
107.6 \\
107.4 \\
201.2\end{array}$ & $\begin{array}{l}2.0 \\
2.0 \\
2.0 \\
2.0 \\
2.0\end{array}$ \\
\hline $\begin{array}{r}10.88 \\
11.38 \\
5.78 \\
44.55 \\
31.55\end{array}$ & $\begin{array}{l}.141 \\
.175 \\
.232 \\
.154 \\
.150\end{array}$ & $\begin{array}{r}.476 \\
.955 \\
.484 \\
1.471 \\
1.743\end{array}$ & $\begin{array}{r}14.4 \\
42.6 \\
6.5 \\
133.2 \\
203.8\end{array}$ & $\begin{array}{l}2.0 \\
2.0 \\
2.0 \\
2.0 \\
2.0\end{array}$ \\
\hline $\begin{array}{l}39.3 \\
23.80 \\
18.45 \\
28.80 \\
14.36\end{array}$ & $\begin{array}{l}.189 \\
.089 \\
.067 \\
.037 \\
.080\end{array}$ & $\begin{array}{r}2.015 \\
1.333 \\
.978 \\
.592 \\
.843\end{array}$ & $\begin{array}{r}168.8 \\
24.1 \\
17.4 \\
18.2 \\
10.3\end{array}$ & $\begin{array}{l}2.0 \\
2.0 \\
2.0 \\
2.0 \\
2.0\end{array}$ \\
\hline $\begin{array}{l}29.80 \\
19.08 \\
22.45 \\
24.30 \\
23.35\end{array}$ & $\begin{array}{l}.078 \\
.111 \\
.096 \\
.121 \\
.088\end{array}$ & $\begin{array}{l}.762 \\
.900 \\
.764 \\
.968 \\
.738\end{array}$ & $\begin{array}{l}44.1 \\
32.4 \\
32.4 \\
32.4 \\
32.4\end{array}$ & $\begin{array}{l}2.0 \\
2.0 \\
2.0 \\
2.0 \\
2.0\end{array}$ \\
\hline $\begin{array}{l}16.82 \\
22.45 \\
32.08 \\
19.32 \\
18.82\end{array}$ & $\begin{array}{l}.083 \\
.073 \\
.082 \\
.085 \\
.080\end{array}$ & $\begin{array}{l}.688 \\
.595 \\
.666 \\
.855 \\
.782\end{array}$ & $\begin{array}{l}32.4 \\
32.4 \\
32.4 \\
46.5 \\
40.6\end{array}$ & $\begin{array}{l}2.0 \\
2.0 \\
2.0 \\
2.0 \\
2.0\end{array}$ \\
\hline $\begin{array}{l}25.55 \\
18.60\end{array}$ & $\begin{array}{r}.072 \\
.148 \\
\end{array}$ & $\begin{array}{l}.753 \\
.741 \\
\end{array}$ & $\begin{array}{l}40.6 \\
30.9 \\
\end{array}$ & $\begin{array}{l}2.0 \\
2.0 \\
\end{array}$ \\
\hline
\end{tabular}

$\dagger$ Adjusted to $\left(k_{2}\right)_{20^{\circ}}$, using equation 104.
TABLE 25. - Reaeration coefficients and hydraulic data from Krenkel (1960)

\begin{tabular}{|c|c|c|c|c|}
\hline $\begin{array}{c}k_{2} \dagger \\
\left(\text { day }^{-1}\right)\end{array}$ & $\begin{array}{c}H \\
\text { (feet) }\end{array}$ & $\begin{array}{c}U \\
\text { (fps) }\end{array}$ & $\begin{array}{c}S \\
\left(\times 10^{4}\right)\end{array}$ & $\begin{array}{c}W \\
\text { (feet) }\end{array}$ \\
\hline $\begin{array}{l}34.37 \\
69.91 \\
37.26 \\
24.66 \\
64.32\end{array}$ & $\begin{array}{r}0.0802 \\
.0823 \\
.0820 \\
.0824 \\
.0861\end{array}$ & $\begin{array}{r}0.473 \\
.739 \\
.385 \\
.295 \\
.788\end{array}$ & $\begin{array}{r}44.38 \\
100.00 \\
28.25 \\
15.75 \\
100.00\end{array}$ & $\begin{array}{l}1.0 \\
1.0 \\
1.0 \\
1.0 \\
1.0\end{array}$ \\
\hline $\begin{array}{l}82.39 \\
99.38 \\
50.93 \\
75.93 \\
40.06\end{array}$ & $\begin{array}{l}.0852 \\
.0857 \\
.0844 \\
.0871 \\
.0894\end{array}$ & $\begin{array}{r}.897 \\
1.020 \\
.634 \\
.823 \\
.461\end{array}$ & $\begin{array}{r}145.88 \\
162.86 \\
70.33 \\
106.75 \\
32.00\end{array}$ & $\begin{array}{l}1.0 \\
1.0 \\
1.0 \\
1.0 \\
1.0\end{array}$ \\
\hline $\begin{array}{c}104.3 \\
115.3 \\
34.51 \\
26.57 \\
100.7\end{array}$ & $\begin{array}{l}.0930 \\
.0901 \\
.0878 \\
.0932 \\
.0876\end{array}$ & $\begin{array}{r}1.330 \\
1.205 \\
.553 \\
.364 \\
1.024\end{array}$ & $\begin{array}{r}239.90 \\
212.50 \\
44.00 \\
17.50 \\
158.75\end{array}$ & $\begin{array}{l}1.0 \\
1.0 \\
1.0 \\
1.0 \\
1.0\end{array}$ \\
\hline $\begin{array}{r}111.7 \\
59.22 \\
78.99 \\
77.25 \\
40.80\end{array}$ & $\begin{array}{l}.0882 \\
.0964 \\
.0971 \\
.0974 \\
.0974\end{array}$ & $\begin{array}{r}1.063 \\
.859 \\
1.020 \\
1.150 \\
.605\end{array}$ & $\begin{array}{r}177.75 \\
93.75 \\
127.50 \\
164.00 \\
45.25\end{array}$ & $\begin{array}{l}1.0 \\
1.0 \\
1.0 \\
1.0 \\
1.0\end{array}$ \\
\hline $\begin{array}{l}91.04 \\
67.25 \\
29.11 \\
66.73 \\
14.31\end{array}$ & $\begin{array}{l}.0963 \\
.1091 \\
.1115 \\
.0993 \\
.1001\end{array}$ & $\begin{array}{r}1.185 \\
1.200 \\
.662 \\
.898 \\
.243\end{array}$ & $\begin{array}{r}137.50 \\
137.50 \\
411.80 \\
91.56 \\
8.00\end{array}$ & $\begin{array}{l}1.0 \\
1.0 \\
1.0 \\
1.0 \\
1.0\end{array}$ \\
\hline $\begin{array}{c}49.39 \\
102.5 \\
114.9 \\
109.2 \\
43.70\end{array}$ & $\begin{array}{l}.1048 \\
.1045 \\
.1090 \\
.1043 \\
.1232\end{array}$ & $\begin{array}{r}.793 \\
1.272 \\
1.590 \\
1.438 \\
.830\end{array}$ & $\begin{array}{r}63.44 \\
175.00 \\
236.00 \\
212.60 \\
51.75\end{array}$ & $\begin{array}{l}1.0 \\
1.0 \\
1.0 \\
1.0 \\
1.0\end{array}$ \\
\hline $\begin{array}{l}62.70 \\
71.81 \\
64.31 \\
96.17 \\
19.65\end{array}$ & $\begin{array}{l}.1182 \\
.1161 \\
.1189 \\
.1360 \\
.1416\end{array}$ & $\begin{array}{r}.967 \\
1.085 \\
1.108 \\
1.590 \\
.539\end{array}$ & $\begin{array}{r}76.25 \\
104.00 \\
100.45 \\
156.25 \\
17.50\end{array}$ & $\begin{array}{l}1.0 \\
1.0 \\
1.0 \\
1.0 \\
1.0\end{array}$ \\
\hline $\begin{array}{l}32.64 \\
41.50 \\
95.67 \\
82.67 \\
13.26\end{array}$ & $\begin{array}{l}.1422 \\
.1415 \\
.1427 \\
.1410 \\
.1447\end{array}$ & $\begin{array}{r}.733 \\
.904 \\
1.450 \\
1.510 \\
.359\end{array}$ & $\begin{array}{r}31.75 \\
49.25 \\
118.25 \\
138.50 \\
7.75\end{array}$ & $\begin{array}{l}1.0 \\
1.0 \\
1.0 \\
1.0 \\
1.0\end{array}$ \\
\hline $\begin{array}{l}53.02 \\
69.10 \\
53.23 \\
96.13 \\
97.18\end{array}$ & $\begin{array}{l}.1463 \\
.1451 \\
.1478 \\
.1521 \\
.1507\end{array}$ & $\begin{array}{l}1.180 \\
1.340 \\
1.030 \\
1.810 \\
2.030\end{array}$ & $\begin{array}{r}77.25 \\
106.00 \\
59.00 \\
180.00 \\
212.60\end{array}$ & $\begin{array}{l}1.0 \\
1.0 \\
1.0 \\
1.0 \\
1.0\end{array}$ \\
\hline $\begin{array}{c}108.7 \\
13.04 \\
38.05 \\
52.01 \\
53.31\end{array}$ & $\begin{array}{l}.1481 \\
.1650 \\
.1651 \\
.1650 \\
.1662\end{array}$ & $\begin{array}{l}2.140 \\
.408 \\
1.050 \\
1.350 \\
1.560\end{array}$ & $\begin{array}{r}234.30 \\
7.50 \\
49.50 \\
78.50 \\
105.75\end{array}$ & $\begin{array}{l}1.0 \\
1.0 \\
1.0 \\
1.0 \\
1.0\end{array}$ \\
\hline $\begin{array}{l}32.32 \\
10.63 \\
16.46 \\
39.18 \\
28.85\end{array}$ & $\begin{array}{l}.1651 \\
.2014 \\
.1983 \\
.1985 \\
.1989\end{array}$ & $\begin{array}{r}.828 \\
.441 \\
.743 \\
1.186 \\
1.020\end{array}$ & $\begin{array}{r}33.25 \\
7.50 \\
18.00 \\
49.25 \\
32.75\end{array}$ & $\begin{array}{l}1.0 \\
1.0 \\
1.0 \\
1.0 \\
1.0\end{array}$ \\
\hline $\begin{array}{l}37.62 \\
42.99 \\
50.54 \\
\end{array}$ & $\begin{array}{l}.1995 \\
.1968 \\
.1989\end{array}$ & $\begin{array}{l}1.315 \\
1.478 \\
1.790\end{array}$ & $\begin{array}{r}59.00 \\
77.00 \\
113.60 \\
\end{array}$ & $\begin{array}{l}1.0 \\
1.0 \\
1.0\end{array}$ \\
\hline
\end{tabular}

†Adjusted to $\left(k_{2}\right)_{20^{\circ}}$, using equation 104 . 